\title{
Generalized Springer correspondence for symmetric spaces associated to orthogonal groups
}

\author{
Toshiaki Shoji and Gao Yang
}

\begin{abstract}
Let $G=G L_{N}(\mathbf{k})$, where $\mathbf{k}$ is an algebraically closed field of ch $\mathbf{k} \neq 2$, and $\theta$ an involutive automorphism of $G$ such that $H=\left(G^{\theta}\right)^{0}$ is isomorphic to $S O_{N}(\mathbf{k})$. Then $G^{\iota \theta}=\left\{g \in G \mid \theta(g)=g^{-1}\right\}$ is regarded as a symmetric space $G / G^{\theta}$. Let $G_{\mathrm{uni}}^{\iota \theta}$ be the set of unipotent elements in $G^{\iota \theta}$. $H$ acts on $G_{\text {uni }}^{\iota \theta}$ by the conjugation. As an analogue of the generalized Springer correspondence in the case of reductive groups, we establish in this paper the generalized Springer correspondence between $H$-orbits in $G_{\mathrm{uni}}^{\iota \theta}$ and irreducible representations of various symmetric groups.
\end{abstract}

\section{INTRODUCTION}

In [L1], Lusztig established the theory of generalized Springer correspondence for reductive groups. Let $G$ be a connected reductive group, and $G_{\text {uni }}$ the set of unipotent elements in $G$. $G_{\text {uni }}$ has finitely many conjugacy classes, and let $\mathscr{N}_{G}$ be the set of pairs $(C, \mathscr{E})$, where $C$ is a unipotent class, and $\mathscr{E}$ is a $G$-equivariant simple $\left(\overline{\mathbf{Q}}_{l^{-}}\right)$ local system on $C$. Lusztig gave a notion of cuspidal pairs for $\mathscr{N}_{G}$, and showed that $\mathscr{N}_{G}$ has a natural partition

$$
\mathscr{N}_{G}=\coprod_{c \in \mathscr{S}_{G}} \mathscr{N}_{G}^{(c)}
$$

Here $\mathscr{S}_{G}$ is the set of isomorphism classes of the triples $\left(L, C_{L}, \mathscr{E}_{L}\right)$, where $L$ is a Levi subgroup of some parabolic subgroup of $G$, and $\left(C_{L}, \mathscr{E}_{L}\right)$ is a cuspidal pair on $L$. He showed that $\mathscr{W}_{L}=N_{G}(L) / L$ is a Coxeter group, and that there exists a natural bijection between $\mathscr{N}_{G}^{(c)}$ and the set $\mathscr{W}_{L}^{\wedge}$ of irreducible representations of $\mathscr{W}_{L}$ over $\overline{\mathbf{Q}}_{l}$, namely,

$$
\mathscr{N}_{G} \simeq \coprod_{\left(L, C_{L}, \mathscr{E}_{L}\right)} \mathscr{W}_{L}^{\wedge}
$$

where $\left(L, C_{L}, \mathscr{E}_{L}\right)$ runs over the classes in $\mathscr{S}_{G} \cdot(*)$ is a generalization of the Springer correspondence, which is a natural injective map $W^{\wedge} \rightarrow \mathscr{N}_{G}$, where $W$ is the Weyl group of $G$. The correspondence $\mathscr{N}_{G}^{(c)} \simeq \mathscr{W}_{L}^{\wedge}$ is obtained by considering a certain semisimple perverse sheaf on $G$ arising from a finite Galois covering related to $\mathscr{W}_{L}$, and by restricting each simple component to $G_{\text {uni }}$.

In this paper, we consider a similar problem for symmetric spaces associated to orthogonal groups. Let $G=G L_{n}(\mathbf{k})$, where $\mathbf{k}$ is an algebraically closed field of odd characteristic. We consider an involutive automorphism $\theta: G \rightarrow G$ such that its subgroup $G^{\theta}$ of $\theta$-fixed points is isomorphic to $O_{N}(\mathbf{k})$. Put $H=\left(G^{\theta}\right)^{0}$. 
Let $\iota: G \rightarrow G$ be the anti-automorphism $g \mapsto g^{-1}$, and consider a closed subset $G^{\iota \theta}=\left\{g \in G \mid \theta(g)=g^{-1}\right\}$ of $G$. As a variety, $G^{\iota \theta}$ is isomorphic to the symmetric space $G / G^{\theta}$. Let $G_{\text {uni }}^{\iota \theta}$ be the set of unipotent elements in $G^{\iota \theta}$. $G^{\iota \theta}$ and $G_{\text {uni }}^{\iota \theta}$ are stable under the conjugation action of $H$ on $G$. It is known that $G_{\text {uni }}^{\iota \theta}$ has finitely many $H$-orbits $([\mathrm{R}])$.

We consider the set $\mathscr{N}_{G}$ of pairs $(\mathscr{O}, \mathscr{E})$, where $\mathscr{O}$ is an $H$-orbit in $G_{\text {uni }}^{\iota \theta}$, and $\mathscr{E}$ is an $H$-equivariant simple local system on $\mathscr{O}$. We can formulate the notion of cuspidal pairs for $\mathscr{N}_{G}$, and so obtain a similar set $\mathscr{S}_{G}$ as before. However some difficulty occurs for constructing a semisimple perverse sheaf on $G^{\iota \theta}$ related to a finite Galois covering. Our discussion is based on the choice of a $\theta$-stable Borel subgroup $B$ and a $\theta$-stable maximal torus $T$ contained in $B$. But in our case, $T^{\iota \theta}$ is not maximal $\theta$-anisotropic, and the role of semisimple elements becomes restricted in contrast to the case of reductive groups (see 1.4). In order to overcome this difficulty, we introduce a certain subgroup $\mathscr{D}$ of $U$ such that $\mathscr{D}$ is a subset of $U^{\iota \theta}$, where $U$ is the unipotent radical of $B$. By making use of $\mathscr{D}$, one can construct a finite Galois covering, and a semisimple perverse sheaf on $G^{\iota \theta}$ associated to it. Once this is done, basically a similar strategy as in [L1] can be applied for the remaining part, and we can establish the generalized Springer correspondence. By making use of the restriction theorem analogous to [L1], we give an explicit description of the generalized Springer correspondence, based on the discussion used in LusztigSpaltenstein [LS] in the case of classical groups.

Let $\mathfrak{g}$ be the Lie algebra of $G$, and $\theta: \mathfrak{g} \rightarrow \mathfrak{g}$ an induced automorphism . The set $\mathfrak{g}_{\text {nil }}^{-}=\left\{x \in \mathfrak{g}_{\text {nil }} \mid \theta(x)=-x\right\}$ of nilpotent elements in $\mathfrak{g}^{-}$is isomorphic to $G_{\text {uni }}^{\iota \theta}$, compatible with $H$-action. One can consider the set $\mathscr{N}_{\mathfrak{g}}$ of pairs $(\mathscr{O}, \mathscr{E})$, where $\mathscr{O}$ is an $H$-orbit in $\mathfrak{g}_{\text {nil }}^{-}$and $\mathscr{E}$ is an $H$-equivariant simple local system on $\mathscr{O}$. In the case where $\mathbf{k}=\mathbf{C}$, Chen-Vilonen-Xue [CVX1] considered a similar problem for $\mathscr{N}_{\mathfrak{g}}$, and determined the Springer correspondence, which is a part of $\mathscr{N}_{\mathfrak{g}}$, by making use of the Fourier-Deligne transform of perverse sheaves on $\mathfrak{g}_{\text {nil }}^{-}$, instead of considering the restriction of perverse sheaves on $G$ to $G_{\text {uni }}$. In [CVX2], they treated the whole of $\mathscr{N}_{\mathfrak{g}}$, but the generalized Springer correspondence is not formulated there, and the explicit correspondence is not determined. In [LY], Lusztig-Yun studied perverse sheaves on $\mathfrak{g}_{\text {nil }}^{-}$associated to arbitrary symmetric spaces, and more generally, associated to $\mathbf{Z} / m \mathbf{Z}$-graded Lie algebras, and established the results closely related to the generalized Springer correspondence. In their case, they also use the Fourier-Deligne transforms, instead of the restriction.

\section{Contents}

Introduction

1. Preliminaries on symmetric spaces

2. Cuspidal local systems

3. Admissible complexes

4. Sheaves on the variety of semisimple orbits

5. Generalized Springer correspondence

6. Restriction theorem

7. Unipotent orbits 
8. Structure of the algebra $\mathscr{A}_{\mathscr{E}_{1}}$

9. Determination of the generalized Springer correspondence

10. Induction

Appendix

\section{Preliminaries on Symmetric SPACES}

1.1. Let $G$ be a connected reductive group over an algebraically closed field $\mathbf{k}$ of ch $\mathbf{k} \neq 2$, and $\theta: G \rightarrow G$ be an involutive automorphism on $G$. Then $G^{\theta}=\{g \in G \mid$ $\theta(g)=g\}$ is a reductive subgroup of $G$. We put $H=\left(G^{\theta}\right)^{0}$. Let $\iota: G \rightarrow G, g \mapsto g^{-1}$ be the anti-automorphism on $G$. We consider the set $G^{\iota \theta}=\left\{g \in G \mid \theta(g)=g^{-1}\right\}$ of $\iota \theta$-fixed points in $G$. Then $G$ acts on $G^{\iota \theta}$ by $g: x \mapsto g x \theta(g)^{-1}$. This action is called the twisted action of $G$. $G$ acts transitively on each connected component of $G^{\iota \theta}$ with respect to the twisted $G$-action $([\mathrm{R}])$. Thus there are only finitely many $G$-orbits in $G^{\iota \theta}$, and each $G$-orbit is closed. In particular, the set $\left\{g \theta(g)^{-1} \mid g \in G\right\}$ is a connected component of $G^{\iota \theta}$.

It is known by [St, $\S 7$ ] that there exist a $\theta$-stable Borel subgroup $B$ and a $\theta$-stable maximal torus $T$ of $G$ such that $B \supset T$. We show a lemma.

Lemma 1.2. Let $(B, T),\left(B^{\prime}, T^{\prime}\right)$ be $\theta$-stable pairs of Borel subgroup and maximal torus of $G$. Assume that $T^{\iota \theta}$ is connected. Then there exists $g \in G^{\theta}$ such that $g B g^{-1}=B^{\prime}, g T g^{-1}=T^{\prime}$.

Proof. We choose $x \in G$ such that $x B x^{-1}=B^{\prime}, x T x^{-1}=T^{\prime}$. Then $\theta(x) B \theta(x)^{-1}=$ $x B x^{-1}, \theta(x) T \theta(x)^{-1}=x T x^{-1}$. Hence $x^{-1} \theta(x) \in B \cap N_{G}(T)=T$. In particular, $x^{-1} \theta(x)=t \in T^{\iota \theta}$. Since $T^{\iota \theta}$ is a torus, one can find $t_{1} \in T^{\iota \theta}$ such that $t=\left(t_{1}\right)^{2}$, i.e., $t=t_{1} \theta\left(t_{1}\right)^{-1}$. If we put $g=x t_{1}$, we have $g \in G^{\theta}$, and $B^{\prime}=g B g^{-1}, T^{\prime}=g T g^{-1}$. The lemma is proved.

Remark 1.3. In general, $\theta$-stable pairs $(B, T),\left(B^{\prime}, T^{\prime}\right)$ are not necessarily $H$ conjugate, (see 1.9).

1.4. We fix a $\theta$-stable Borel subgroup $B$ and a $\theta$-stable maximal torus $T$ such that $T \subset B$. A torus $S$ is called $\theta$-anisotropic if $\theta(t)=t^{-1}$ for any $t \in S$. A maximal $\theta$-anisotropic tours is a $\theta$-anisotropic torus which is maximal with respect to the inclusion relation. It is known by $[\mathrm{V}]$ that a $\theta$-anisotropic torus exists if $\theta \neq \mathrm{id}$, and every maximal $\theta$-anisotropic tori are conjugate under $H$. Moreover, any semisimple element in $G^{\iota \theta}$ is contained in some maximal $\theta$-anisotropic torus. Here $\left(T^{\llcorner\theta}\right)^{0}$ is a $\theta$-anisotropic torus, but in general, it is not maximal $\theta$-anisotropic as the following example shows.

Let $G=G L_{2}$ and define $\theta: G \rightarrow G$ by $\theta(g)=J^{-1}\left({ }^{t} g^{-1}\right) J$ with $J=\left(\begin{array}{ll}0 & 1 \\ 1 & 0\end{array}\right)$.

We define $x \in G$ by

$$
x=\left(\begin{array}{cc}
1 & -\zeta \\
1 / 2 & \zeta / 2
\end{array}\right)
$$

where $\zeta \in \mathbf{k}^{*}$ such that $\zeta^{2}=-1$. One can check that $x^{-1} \theta(x)=J$. Let $T$ be the group of diagonal matrices, and $B$ the group of upper triangular matrices in $G$. 
Then $(B, T)$ is a $\theta$-stable pair of Borel subgroup and maximal torus of $G$. In this case,

$$
T^{\iota \theta}=\left\{\left(\begin{array}{ll}
a & 0 \\
0 & a
\end{array}\right) \mid a \in \mathbf{k}^{*}\right\}=Z(G) .
$$

Put $T_{1}=x T x^{-1}$. Since $x^{-1} \theta(x)=J \in N_{G}(T), T_{1}$ is also a $\theta$-stable maximal torus. One can check that $T_{1}^{\iota \theta}=T_{1}$, and so $T_{1}$ is a maximal $\theta$-anisotropic torus. Since $\operatorname{dim} T^{\iota \theta}=1, \operatorname{dim} T_{1}=2, T^{\iota \theta}$ is not maximal.

1.5. Put $B_{H}=B \cap H$, and $T_{H}=T \cap H$. Then as remarked in [R, Lemma 5.1], $B_{H}$ is a Borel subgroup of $H$ and $T_{H}$ is a maximal torus of $H$ contained in $B_{H}$. Let $\mathfrak{g}=$ Lie $G$ be the Lie algebra of $G$, and put $\mathfrak{b}=\operatorname{Lie} B, \mathfrak{t}=\operatorname{Lie} T$. The differential $d \theta$ of $\theta$ induces an involutive automorphism of $\mathfrak{g}$, which we also denote by $\theta$. Thus, $\mathfrak{b}, \mathfrak{t}$ are $\theta$-stable. We have a decomposition $\mathfrak{g}=\mathfrak{g}^{+} \oplus \mathfrak{g}^{-}$, where $\mathfrak{g}^{ \pm}=\{x \in \mathfrak{g} \mid \theta(x)= \pm x\}$. Here $\mathfrak{g}^{+}=$Lie $H$, and $\mathfrak{g}^{-}$coincides with the tangent space of $G / G^{\theta} \simeq G^{\iota \theta}$. Let $\Delta \subset X(T)$ be the root system of $G$ with respect to $T$ (here $X(T)$ is the character group of $T$ ), and $\Delta_{+}$the set of positive roots in $\Delta$ with respect to $(T, B)$. We have the root space decomposition $\mathfrak{g}=\mathfrak{t} \oplus \bigoplus_{\alpha \in \Delta} \mathfrak{g}_{\alpha}$. The map $\alpha \mapsto \alpha \circ \theta$ on $X(T)$ induces a bijection on $\Delta$, which we denote by $\sigma . \sigma: \Delta \rightarrow \Delta$ is compatible with the root space decomposition, and $\theta$ gives an isomorphism $\theta: \mathfrak{g}_{\alpha} \rightarrow \mathfrak{g}_{\sigma(\alpha)} . \sigma$ preserves $\Delta_{+}$, and so produces an automorphism of the Dynkin diagram of $G$. Put

$$
\Delta_{0}=\left\{\alpha \in \Delta_{+} \mid \sigma(\alpha)=\alpha\right\}
$$

$\sigma$ acts freely on $\Delta_{1}=\Delta_{+}-\Delta_{0}$, and we denote by $\bar{\Delta}_{1}$ the set of $\sigma$-orbits in $\Delta_{1}$. For each $\beta=\{\alpha, \sigma(\alpha)\} \in \bar{\Delta}_{1}$, put $\mathfrak{g}_{\beta}=\mathfrak{g}_{a} \oplus \mathfrak{g}_{\sigma(\alpha)}$. Then $\mathfrak{g}_{\beta}$ is $\theta$-stable, and $\mathfrak{g}_{\beta}$ is decomposed as $\mathfrak{g}_{\beta}=\mathfrak{g}_{\beta}^{+} \oplus \mathfrak{g}_{\beta}^{-}$with $\mathfrak{g}_{\beta}^{+} \subset \mathfrak{g}^{+}, \mathfrak{g}_{\beta}^{-} \subset \mathfrak{g}^{-}$. If $\alpha \in \Delta_{0}$, then $\theta$ gives a linear isomorphism $\mathfrak{g}_{\alpha} \rightarrow \mathfrak{g}_{\alpha}$. Since $\theta^{2}=1$, we have $\theta= \pm 1$ on $\mathfrak{g}_{\alpha}$, i.e., $\mathfrak{g}_{\alpha} \subset \mathfrak{g}^{+}$or $\mathfrak{g}_{\alpha} \subset \mathfrak{g}^{-}$. We denote by $\Delta_{0}^{+}$(resp. $\Delta_{0}^{-}$) the set of $\alpha \in \Delta_{0}$ such that $\mathfrak{g}_{\alpha} \subset \mathfrak{g}^{+}$(resp. $\mathfrak{g}_{\alpha} \subset \mathfrak{g}^{-}$). Let $\mathfrak{n}$ be the nilpotent radical of $\mathfrak{b}$. $\mathfrak{n}$ is $\theta$-stable, and is decomposed as $\mathfrak{n}=\mathfrak{n}^{+} \oplus \mathfrak{n}^{-}$, where $\mathfrak{n}^{ \pm}=\mathfrak{n} \cap \mathfrak{g}^{ \pm}$. We have a root space decomposition

$$
\mathfrak{n}^{ \pm}=\bigoplus_{\beta \in \bar{\Delta}_{1}} \mathfrak{g}_{\beta}^{ \pm} \oplus \bigoplus_{\alpha \in \Delta_{0}^{ \pm}} \mathfrak{g}_{\alpha}
$$

1.6. Let $U$ be the unipotent radical of $B$, which is $\theta$-stable, and put $U_{H}=$ $U^{\theta}=U \cap H$. For each $\alpha \in \Delta_{+}$, we have a one parameter subgroup $U_{\alpha} \subset U$ such that Lie $U_{\alpha}=\mathfrak{g}_{\alpha}$. We have an isomorphism

$$
U \simeq \prod_{\alpha \in \Delta_{+}} U_{\alpha}
$$

for a choice of the total order on $\Delta_{+}$. Here $\theta: U \rightarrow U$ induces an isomorphism $\theta: U_{\alpha} \rightarrow U_{\sigma(a)}$ for each $\alpha$, and according to the decomposition in (1.5.2) in the Lie 
algebra case, we have

$$
U_{H} \simeq \prod_{\{\alpha, \sigma(\alpha)\} \in \bar{\Delta}_{1}}\left(U_{\alpha} U_{\sigma(\alpha)}\right)^{\theta} \times \prod_{\alpha \in \Delta_{0}^{+}} U_{\alpha} .
$$

Note that $U\left(\Delta_{0}\right)=\prod_{\alpha \in \Delta_{0}} U_{\alpha}$ is a $\theta$-stable closed subgroup of $U$, and its $\theta$-fixed point subgroup coincides with $\prod_{\alpha \in \Delta_{0}^{+}} U_{\alpha}$.

Concerning $U^{\iota \theta}$, we obtain a similar description as in (1.6.1). But since $\iota \theta$ is not a group homomorphism, we need a special care. One can find a filtration $U \supset U_{1} \supset$ $U_{2} \supset \cdots$ of $U$ by $\theta$-stable normal subgroups, by making use of the commutator relations for $U$, such that each quotient group $U_{i} / U_{i+1}$ is of the form $U_{\alpha} U_{\sigma(\alpha)}$ for $\{\alpha, \sigma(\alpha)\} \in \bar{\Delta}_{1}$ or of the form $U_{\alpha}$ for $\alpha \in \Delta_{0}^{-}$. In that case, $U^{\iota \theta}$ has a filtration $U^{\iota \theta} \supset U_{1}^{\iota \theta} \supset U_{2}^{\iota \theta} \supset \cdots$ (by affine subspaces) such that $U_{i}^{\iota \theta} / U_{i+1}^{\iota \theta} \simeq\left(U_{\alpha} U_{\sigma(\alpha)}\right)^{\iota \theta}$ or $\simeq U_{\alpha}^{\iota \theta}$ accordingly. In particular, $U_{H}$ (resp. $\left.U^{\iota \theta}\right)$ is isomorphic to an affine space with $\operatorname{dim} U_{H}=\operatorname{dim} \mathfrak{n}^{+}\left(\right.$resp. $\left.\operatorname{dim} U^{\iota \theta}=\operatorname{dim} \mathfrak{n}^{-}\right)$.

1.7. In the remaining part of the paper, we concentrate on the symmetric spaces associated to orthogonal groups. Let $V$ be an $N$ dimensional vector space over $\mathbf{k}$, and let $G=G L_{N}=G L(V)$ with $N \geq 2$. Consider an involutive automorphism $\theta: G \rightarrow G$ defined by $\theta(g)=J^{-1}\left({ }^{t} g^{-1}\right) J$, where

$$
\begin{aligned}
& J=\left(\begin{array}{ccc}
1 & 0 & 0 \\
0 & 0 & 1_{n} \\
0 & 1_{n} & 0
\end{array}\right) \quad \text { if } N=2 n+1, \\
& J=\left(\begin{array}{cc}
0 & 1_{n} \\
1_{n} & 0
\end{array}\right) \quad \text { if } N=2 n,
\end{aligned}
$$

with $1_{n}$ the identity matrix of degree $n$. Let $H=\left(G^{\theta}\right)^{0}$. Then $H$ is a special orthogonal group $S O_{N}$ with respect to the symmetric bilinear form $\langle u, v\rangle={ }^{t} u J v$ on $V(u, v \in V)$, under the identification $V \simeq \mathbf{k}^{N}$ via the basis $\left\{e_{0}, e_{1}, \ldots, e_{n}, f_{1}, \ldots f_{n}\right\}$ in the case where $N=2 n+1$, and the basis $\left\{e_{1}, \ldots, e_{n}, f_{1}, \ldots, f_{n}\right\}$ in the case where $N=2 n$. It follows that

$$
\begin{aligned}
& \left\langle e_{i}, f_{j}\right\rangle=\left\langle f_{j}, e_{i}\right\rangle=\delta_{i, j} \text { for } 1 \leq i, j \leq n, \\
& \left\langle e_{i}, e_{j}\right\rangle=\left\langle f_{i}, f_{j}\right\rangle=0 \text { for } 1 \leq i, j \leq n,
\end{aligned}
$$

and $\left\langle e_{0}, e_{0}\right\rangle=1,\left\langle e_{0}, e_{i}\right\rangle=\left\langle e_{0}, f_{i}\right\rangle=0$ for $i \geq 1$.

Let $\mathfrak{g}=\operatorname{Lie} G$, and $\theta: \mathfrak{g} \rightarrow \mathfrak{g}$ be the induced automorphism. Then $\theta(x)=$ $-J^{-1}\left({ }^{t} x\right) J$ for $x \in \mathfrak{g}$. Let $x^{*}$ be the adjoint of $x \in \mathfrak{g}$ with respect to the bilinear form $\langle$,$\rangle . Then we have x^{*}=J^{-1}\left({ }^{t} x\right) J$, and so

$$
\mathfrak{g}^{ \pm}=\left\{x \in \mathfrak{g} \mid x^{*}=\mp x\right\} .
$$

We have 


$$
\begin{aligned}
& \mathfrak{g}^{+}=\{x \in \mathfrak{g} \mid\langle x v, w\rangle=-\langle v, x w\rangle\} \\
& \mathfrak{g}^{-}=\{x \in \mathfrak{g} \mid\langle x v, w\rangle=\langle v, x w\rangle\} .
\end{aligned}
$$

In particular, $\mathfrak{g}^{-}$coincides with the set of self-adjoint matrices in $\mathfrak{g}=\mathfrak{g l}_{N}$. Correspondingly, $G^{\iota \theta}$ coincides with the set of non-degenerate self-adjoint matrices in $\mathfrak{g l}_{N}$. In particular, $G^{\iota \theta}$ is connected, and by 1.1 we have

$$
G^{\iota \theta}=\left\{g \theta(g)^{-1} \mid g \in G\right\}
$$

1.8. We fix a $\theta$-stable Borel subgroup $B$ and a $\theta$-stable maximal torus $T \subset B$ as follows. First assume that $N=2 n+1$. Let $B$ be the subgroup of $G$ consisting of the matrices of the form

$$
\left(\begin{array}{ccc}
a & 0 & d_{1} \\
{ }^{t} d_{2} & b_{1} & c \\
0 & 0 & b_{2}
\end{array}\right)
$$

where $a \in \mathbf{k}^{*}, d_{1}, d_{2}$ are (row) vectors in $\mathbf{k}^{n}$, and $b_{1}, b_{2}, c$ are square matrices of degree $n$ with $b_{1}$ upper triangular, $b_{2}$ lower triangular. Let $T$ be the set of all diagonal matrices in $G$. Then $B$ is a Borel subgroup of $G$, and $T$ is a maximal torus in $G$ with $T \subset B$. $B, T$ are both $\theta$-stable. We have

$$
T^{\theta}=\left\{\left(\begin{array}{ccc} 
\pm 1 & 0 & 0 \\
0 & b & 0 \\
0 & 0 & b^{-1}
\end{array}\right) \mid b \in D_{n}\right\}, \quad T^{\iota \theta}=\left\{\left(\begin{array}{ccc}
a & 0 & 0 \\
0 & b & 0 \\
0 & 0 & b
\end{array}\right) \mid a \in \mathbf{k}^{*}, b \in D_{n}\right\}
$$

where $D_{n}$ is the group of diagonal matrices of $G L_{n}$. Moreover, we have

$$
\begin{aligned}
B^{\theta} & =\left\{\left(\begin{array}{ccc}
a & 0 & d_{1} \\
{ }^{t} d_{2} & b & c \\
0 & 0 & { }^{t} b^{-1}
\end{array}\right) \in B \mid a= \pm 1, d_{2}=-a^{-1} d_{1}{ }^{t} b,{ }^{t} c=-\left({ }^{t} d_{1} d_{1}+b^{-1} c\right)^{t} b\right\} \\
B^{\iota \theta} & =\left\{\left(\begin{array}{ccc}
a & 0 & d \\
{ }^{t} d & b & c \\
0 & 0 & { }^{t} b
\end{array}\right) \in B \mid{ }^{t} c=c\right\} .
\end{aligned}
$$

Next assume that $N=2 n$. Let $B$ be the subgroup of $G$ consisting of the matrices of the form

$$
\left(\begin{array}{cc}
b_{1} & c \\
0 & b_{2}
\end{array}\right)
$$

where $b_{1}, b_{2}, c$ are square matrices of degree $n$ with $b_{1}$ upper triangular, $b_{2}$ lower triangular. Let $T$ be the set of diagonal matrices in $G$. Then $B$ is a Borel subgroup of $G$ and $T$ is a maximal torus in $G$ with $T \subset B$. B, $T$ are both $\theta$-stable. We have 


$$
T^{\theta}=\left\{\left(\begin{array}{cc}
b & 0 \\
0 & b^{-1}
\end{array}\right) \mid b \in D_{n}\right\}, \quad T^{\iota \theta}=\left\{\left(\begin{array}{ll}
b & 0 \\
0 & b
\end{array}\right) \mid b \in D_{n}\right\} .
$$

Moreover, we have

$$
\begin{aligned}
B^{\theta} & =\left\{\left(\begin{array}{cc}
b & c \\
0 & { }^{t} b^{-1}
\end{array}\right) \in B \mid{ }^{t} c=-b^{-1} c^{t} b\right\} \\
B^{\iota \theta} & =\left\{\left(\begin{array}{cc}
b & c \\
0 & { }^{t} b
\end{array}\right) \in B \mid{ }^{t} c=c\right\} .
\end{aligned}
$$

Let $U$ be the unipotent radical of $B$. Then $U$ is $\theta$-stable. Put $B_{H}=\left(B^{\theta}\right)^{0}, T_{H}=$ $\left(T^{\theta}\right)^{0}$ and $U_{H}=U^{\theta}$. Thus $B_{H} \supset T_{H}$ is a pair of a Borel subgroup and a maximal torus in $H$, and $U_{H}$ is the unipotent radical of $B_{H}$.

1.9. Let $T \subset B$ be as above. For $i=1, \ldots, n-1$, let $s_{i}$ be the permutation of the basis in $V$ such that $e_{i} \leftrightarrow e_{i+1}, f_{i} \leftrightarrow f_{i+1}$ and that it fixes all other basis. Also for $i=1, \ldots, n$, let $t_{i}$ be the permutation $e_{i} \leftrightarrow f_{i}$ which fixes all other basis. Assume that $N$ is odd. Then $N_{H}(T)$ is generated by $s_{1}, \ldots, s_{n-1}, t_{n}$ and $T . \quad N_{H}(T) / T$ is isomorphic to the Weyl group $W_{n}$ of type $B_{n}$. On the other hand, $N_{G^{\theta}}(T)$ is generated by $N_{H}(T)$ and -1 , so that $N_{G^{\theta}}(T) / T \simeq W_{n} \times \mathbf{Z} / 2 \mathbf{Z}$. Hence in view of Lemma 1.2 (note that $T^{\iota \theta}$ is connected), any $\theta$-stable pair $\left(B^{\prime}, T^{\prime}\right)$ is $H$-conjugate to $(B, T)$.

Next assume that $N$ is even. In this case, $N_{H}(T)$ is generated by $s_{1}, \ldots, s_{n-1}$, $t_{n-1} t_{n}$ and $T . N_{H}(T) / T$ is isomorphic to the Weyl group $W_{n}^{\prime}$ of type $D_{n}$. Moreover, $N_{G^{\theta}}(T)$ is generated by $N_{H}(T)$ and $t_{n}$, hence $N_{G^{\theta}}(T) / T \simeq W_{n}$. One can check that $B_{1}=t_{n} B t_{n}^{-1}$ is not contained in the set of $W_{n}^{\prime}$-conjugates of $B$. It follows that the $\theta$-stable pair $\left(B_{1}, T\right)$ is not $H$-conjugate to $(B, T)$, and $(B, T),\left(B_{1}, T\right)$ give representatives of $H$-conjugates of $\theta$-stable pairs in $G$.

1.10. Let $\Delta$ and $\Delta_{+}$be as in 1.5 with respect to the pair $(B, T)$ in 1.8 . Let $\mathfrak{t}=$ Lie $T$, and fix the basis of $\mathfrak{t}^{*}$ as $\left\{\varepsilon_{0}, \varepsilon_{1}, \ldots, \varepsilon_{n}, \varepsilon_{1}^{\prime}, \ldots, \varepsilon_{n}^{\prime}\right\}$ in the case where $N=2 n+$ 1 , and $\left\{\varepsilon_{1}, \ldots, \varepsilon_{n}, \varepsilon_{1}^{\prime}, \ldots, \varepsilon_{n}^{\prime}\right\}$ in the case where $N=2 n$. Here $\varepsilon_{i}$ (resp. $\varepsilon_{j}^{\prime}, \varepsilon_{0}$ ) is the weight vector corresponding to $e_{i}$ (resp. $\left.f_{j}, e_{0}\right)$. We write those vectors as $\widetilde{\varepsilon}_{1}, \ldots, \widetilde{\varepsilon}_{N}$, in the order $\varepsilon_{1}, \ldots, \varepsilon_{n}, \varepsilon_{0}, \varepsilon_{n}^{\prime}, \ldots, \varepsilon_{1}^{\prime}$ if $N=2 n+1$, and $\varepsilon_{1}, \ldots, \varepsilon_{n}, \varepsilon_{n}^{\prime}, \ldots, \varepsilon_{1}^{\prime}$ if $N=2 n$. Thus $\Delta_{+}$can be written as

$$
\Delta_{+}=\left\{\widetilde{\varepsilon}_{i}-\widetilde{\varepsilon}_{j} \mid 1 \leq i<j \leq N\right\} .
$$

It follows from the description of $B^{\iota \theta}$ in 1.8 , we see that

$$
\Delta_{0}^{+}=\emptyset, \quad \Delta_{0}^{-}=\left\{\widetilde{\varepsilon}_{i}-\widetilde{\varepsilon}_{N-i+1} \mid 1 \leq i \leq n\right\} .
$$

Recall that $U\left(\Delta_{0}\right)=\prod_{\alpha \in \Delta_{0}} U_{\alpha}$ is a $\theta$-stable subgroup of $U$. It follows from (1.10.1), we have $U\left(\Delta_{0}\right)=U\left(\Delta_{0}\right)^{\iota \theta}$, which we denote by $\mathscr{D}$. By using (1.10.1), $\mathscr{D}$ 
can be written explicitly as follows.

$$
\begin{array}{ll}
\mathscr{D}=\left\{\left(\begin{array}{ccc}
1 & 0 & 0 \\
0 & 1_{n} & c \\
0 & 0 & 1_{n}
\end{array}\right) \in U \mid c: \text { diagonal }\right\} & (N=2 n+1), \\
\mathscr{D}=\left\{\left(\begin{array}{cc}
1_{n} & c \\
0 & 1_{n}
\end{array}\right) \in U \mid c: \text { diagonal }\right\} & (N=2 n) .
\end{array}
$$

Thus $\mathscr{D}$ is $T^{\theta}$-stable, and $\mathscr{D} \simeq \mathbf{k}^{n}$.

1.11. Let $P=L U_{P}$ be a $\theta$-stable parabolic subgroup of $G$ containing $B$, where $L$ is the Levi subgroup of $P$ containing $T$ and $U_{P}$ is the unipotent radical of $P$. Here we consider the special case where $L^{\theta} \simeq\left(G L_{1}\right)^{a} \times G L_{N_{0}}^{\theta}$ with $N_{0}=N-2 a$ for $0 \leq a \leq n$ (we understand that $G L_{1}^{\theta}=\{ \pm 1\}, G L_{0}^{\theta}=\{1\}$ ). Put $\mathscr{D}_{P}=\mathscr{D} \cap U_{P}$. Then $\mathscr{D}_{P}$ is a closed subgroup of $U_{P}$, and is contained in $U_{P}^{\iota \theta}$. We have $\mathscr{D}_{P} \simeq \mathbf{k}^{a}$. It is easy to see that $\mathscr{D}_{P}$ is stable under the conjugation action of $L$ on $U_{P}$. More precisely, we have

(1.11.1) Assume that $x \in L^{\iota \theta}$ and $u \in \mathscr{D}_{P}$. Then we have $x u=u x$.

The proof is done by a direct computation by using (1.10.2). Note that in the Lie algebra case, this corresponds to the fact that if $x, y \in \mathfrak{g}^{-}$such that $[x, y] \in \mathfrak{g}^{-}$, then $[x, y]=0$ as $[x, y] \in \mathfrak{g}^{+}$.

Remark 1.12. For the comparison, we briefly discuss the case of symplectic groups. Let $V$ be an $N=2 n$-dimensional vector space over $\mathbf{k}$, and let $G=G L_{N}=$ $G L(V)$. The involutive automorphism $\theta: G \rightarrow G$ is defined as in 1.7, but by

replacing $J$ by $J=\left(\begin{array}{cc}0 & 1_{n} \\ -1_{n} & 0\end{array}\right)$. Then $H=G^{\theta}$ is the symplectic group $S p_{N}$, and we consider $G^{\iota \theta}$ with $H$-action. We choose a pair of $\theta$-stable Borel subgroup $B$ and a $\theta$-stable maximal torus $T \subset B$ as in [SS, 1]. Then the root system $\Delta$ of $G$ and the set of positive roots with respect to the pair $(B, T)$ are given similarly to 1.10 . In particular, the set $\Delta_{0}=\Delta_{0}^{+} \cup \Delta_{0}^{-}$is defined, and $\Delta_{0}^{ \pm}$are determined by using the computation in $[\mathrm{SS}, 1]$, namely, we have

$$
\Delta_{0}^{+}=\left\{\widetilde{\varepsilon}_{i}-\widetilde{\varepsilon}_{N-i+1} \mid 1 \leq i \leq n\right\}, \quad \Delta_{0}^{-}=\emptyset .
$$

(1.12.1) shows that in the symplectic case, an opposite situation occurs compared to the orthogonal case (1.10.1).

\section{Cuspidal local systems}

2.1. In this section, we extend the notion of cuspidal local systems in the case of reductive groups given in [L1] to the case of symmetric spaces. Let $G=G L_{N}$, and $\theta: G \rightarrow G$ be as in 1.7. Let $H=\left(G^{\theta}\right)^{0}$. We denote the twisted action of $G$ on $G^{\iota \theta}$ given by $g: x \mapsto g x \theta(g)^{-1}\left(g \in G, x \in G^{\iota \theta}\right)$ by $g \cdot x$. 
Let $P=L U_{P}$ be a $\theta$-stable parabolic subgroup of $G$ containing $B$, where $L$ is the $\theta$-stable Levi subgroup containing $T$ and $U_{P}$ is the unipotent radical of $P$. Then $L^{\theta} \simeq G L_{n_{1}} \times \cdots \times G L_{n_{a}} \times G L_{N_{0}}^{\theta}$, where $N_{0}+\sum_{i=1}^{a} 2 n_{i}=N$. We have

$$
L^{\iota \theta} \simeq G L_{n_{1}} \times \cdots \times G L_{n_{a}} \times G L_{N_{0}}^{\iota \theta}
$$

on which $L^{\theta}$ acts via the conjugation action. The natural projection $P \rightarrow P / U_{P} \simeq L$ induces a surjective map $\eta_{P}: P^{\iota \theta} \rightarrow L^{\iota \theta}$. Note that $L^{\theta}$ acts on $U_{P}^{\iota \theta}$ by conjugation action. Since $L^{\iota \theta}$ is connected by (2.1.1), we have an isomorphism $L^{\iota \theta} \simeq L / L^{\theta}$ by 1.1. We note the following.

(2.1.2) The map $L \times U_{P}^{\iota \theta} \rightarrow P^{\iota \theta},(\ell, u) \mapsto \ell \cdot u$ induces an isomorphism $L \times{ }^{L^{\theta}} U_{P}^{\iota \theta} \simeq$ $P^{\iota \theta}$. Moreover, the map $\eta_{P}$ is regarded as an affine space bundle over $L^{\iota \theta}$ through the maps

$$
P^{\iota \theta} \simeq L \times^{L^{\theta}} U_{P}^{\iota \theta} \rightarrow L / L^{\theta} \simeq L^{\iota \theta}
$$

where for $g=\ell \cdot u \in P^{\iota \theta}, \eta_{P}(g)=\ell \theta(\ell)^{-1}$.

In fact, take $x \in P^{\iota \theta}$, and write it as $x=\ell_{1} u_{1}$ with $\ell_{1} \in L, u_{1} \in U_{P}$. Then we have $\theta\left(\ell_{1}\right)=\ell_{1}^{-1}, \theta\left(u_{1}\right)=\ell_{1} u_{1}^{-1} \ell_{1}^{-1}$. Since $\ell_{1} \in L^{\iota \theta}$, there exists $\ell \in L$ such that $\ell_{1}=\ell \theta(\ell)^{-1}$. If we put $u=\theta(\ell)^{-1} u_{1} \theta(\ell)$, one can check that $u \in U_{P}^{\iota \theta}$, and we have

$$
x=\ell_{1} u_{1}=\ell\left(\theta(\ell)^{-1} u_{1} \theta(\ell)\right) \theta(\ell)^{-1}=\ell u \theta(\ell)^{-1} .
$$

The pair $(\ell, u)$ is uniquely determined from $g$ modulo the action of $L^{\iota \theta}, y:(\ell, u) \mapsto$ $\left(\ell y^{-1}, y u y^{-1}\right)$ for $y \in L^{\iota \theta}$. Hence (2.1.2) holds.

Put $P_{H}=\left(P^{\theta}\right)^{0}$ and $L_{H}=\left(L^{\theta}\right)^{0}$. Then $P_{H}$ is the parabolic subgroup of $H$ containing $B_{H}$. Let $W_{H}=N_{H}\left(T_{H}\right) / T_{H}$ be the Weyl group of $H$. We define a Weyl subgroup $W_{L_{H}}$ of $W_{H}$ as the Weyl group of $L_{H}$. Let $\mathscr{O}$ be an $H$-orbit in $G^{\iota \theta}$ and $\mathscr{O}_{L}$ an $L_{H^{-}}$orbit in $L^{\iota \theta}$. Let $Z_{L}$ be the center of $L$. Then $Z_{L}^{\iota \theta} \simeq\left(G L_{1}\right)^{a}$.

Consider the varieties

$$
\begin{gathered}
Z=\left\{\left(x, g P_{H}, g^{\prime} P_{H}\right) \in G^{\iota \theta} \times H / P_{H} \times H / P_{H}\right. \\
\left.\mid g^{-1} x g \in \eta_{P}^{-1}\left(Z_{L}^{\iota \theta} \mathscr{O}_{L}\right), g^{\prime-1} x g^{\prime} \in \eta_{P}^{-1}\left(Z_{L}^{\iota \theta} \mathscr{O}_{L}\right)\right\} \\
Z^{\prime}=\left\{\left(x, g P_{H}, g^{\prime} P_{H}\right) \in G^{\iota \theta} \times H / P_{H} \times H / P_{H}\right. \\
\left.\mid g^{-1} x g \in \eta_{P}^{-1}\left(\mathscr{O}_{L}\right), g^{\prime-1} x g^{\prime} \in \eta_{P}^{-1}\left(\mathscr{O}_{L}\right)\right\}
\end{gathered}
$$

We consider the partition $H / P_{H} \times H / P_{H}=\coprod \mathbb{O}_{\omega}$ into $H$-orbits, where $\mathbb{O}_{\omega}$ is an $H$-orbit containing $\left(P_{H}, \omega P_{H}\right)$ for $\omega \in W_{L_{H}} \backslash W_{H} / W_{L_{H}}$. Let $Z_{\omega}=p^{-1}\left(\mathbb{O}_{\omega}\right), Z_{\omega}^{\prime}=$ $p^{\prime-1}\left(\mathbb{O}_{\omega}\right)$, where $p: Z \rightarrow H / P_{H} \times H / P_{H}, p^{\prime}: Z^{\prime} \rightarrow H / P_{H} \times H / P_{H}$ are projections onto second and third factors. An orbit $\mathbb{O}_{\omega}$ is said to be good if $P_{H}$ and $\omega P_{H} \omega^{-1}$ have a common Levi subgroup, otherwise $\mathbb{O}_{\omega}$ is said to be bad. The good orbit corresponds to $\omega$ such that $\omega W_{L_{H}}=W_{L_{H}} \omega$.

In order to proceed the induction process smoothly for proving the proposition below, we replace $G$ by groups appearing as a Levi subgroup of some parabolic 
subgroup of $G$, namely we consider

$$
G=G_{0} \times \prod_{i=1}^{t}\left(G_{i} \times G_{i}\right)
$$

where $G_{0}=G L_{N}, G_{i}=G L_{N_{i}}$. We consider an involutive automorphism $\theta: G \rightarrow G$ such that $\theta$ acts on $G_{i} \times G_{i}$ as a permutation of factors, and $\theta$ acts on $G_{0}$ so that $G_{0}^{\theta} \simeq O_{N}$. Hence

$$
G^{\iota \theta} \simeq G_{0}^{\iota \theta} \times \prod_{i=1}^{t} G_{i}
$$

Under this setting, $P=L U_{P}$ and the varieties $Z, Z^{\prime}$ are defined similarly. In particular, $L^{\theta}$ can be written as $L^{\theta} \simeq G L_{n_{1}} \times \cdots \times G L_{n_{a}} \times G L_{N_{0}}^{\theta}$, where $G L_{N_{0}}^{\theta}$ is a subgroup of $G_{0}^{\theta}$. Put $\nu_{H}=\operatorname{dim} U^{\theta}$, and $\bar{\nu}=\nu_{L_{H}}$. Let $c=\operatorname{dim} \mathscr{O}$ and $\bar{c}=\operatorname{dim} \mathscr{O}_{L}$. Put $r=\operatorname{dim} Z_{L}^{\iota \theta}$. We define $\Delta_{P}$ by

$$
\Delta_{P}=\left(N-N_{0}\right) / 2
$$

The following result is an extension of [L1, Proposition 1.2]. The proof is done along a similar line. But since the Levi decomposition $P=L U_{P}$ does not hold for $P^{\iota \theta}$, we need a special care for it. The discussion based on the Levi decomposition in [L1] will be replaced by the discussion using (2.1.2).

Proposition 2.2. Under the notation above,

(i) For $\bar{x} \in \mathscr{O}_{L}$, we have $\operatorname{dim}\left(\mathscr{O} \cap \eta_{P}^{-1}(\bar{x})\right) \leq(c-\bar{c}) / 2+\Delta_{P} / 2$.

(ii) For $x \in \mathscr{O}$,

$$
\operatorname{dim}\left\{g P_{H} \in H / P_{H} \mid g^{-1} x g \in \eta_{P}^{-1}\left(\mathscr{O}_{L}\right)\right\} \leq\left(\nu_{H}-c / 2\right)-(\bar{\nu}-\bar{c} / 2)+\Delta_{P} / 2 .
$$

(iii) Put $d=2 \nu_{H}-2 \bar{\nu}+\bar{c}+r$. Then $\operatorname{dim} Z_{\omega} \leq d+\Delta_{P}$ if $\mathbb{O}_{\omega}$ is good, and $\operatorname{dim} Z_{\omega}<d+\Delta_{P}$ if $\mathbb{O}_{\omega}$ is bad. Hence $\operatorname{dim} Z \leq d+\Delta_{P}$.

(iv) Put $d^{\prime}=2 \nu_{H}-2 \bar{\nu}+\bar{c}$. Then $\operatorname{dim} Z_{\omega}^{\prime} \leq d^{\prime}+\Delta_{P}$ for any $\omega$. Hence $\operatorname{dim} Z^{\prime} \leq$ $d^{\prime}+\Delta_{P}$

Proof. Since the proposition holds in the case where $G=T \times T$ for a torus $T$, we may assume that the proposition holds for a proper Levi subgroup $L$ of $G$.

Consider an orbit $\mathbb{O}_{\omega}$. Let $w \in W_{H}$ be a representative of $\omega \in W_{L_{H}} \backslash W_{H} / W_{L_{H}}$, and $\dot{w} \in N_{H}\left(T_{H}\right)$ a representative of $w$. In order to show (iii) and (iv), it is enough to see that

$$
\begin{gathered}
\operatorname{dim}\left\{x \in \eta_{P}^{-1}\left(Z_{L}^{\iota \theta} \mathscr{O}_{L}\right) \mid \dot{w}^{-1} x \dot{w} \in \eta_{P}^{-1}\left(Z_{L}^{\iota \theta} \mathscr{O}_{L}\right)\right\} \\
\quad \leq 2 \nu_{H}-2 \bar{\nu}+\bar{c}+r-\operatorname{dim} \mathbb{O}_{\omega}+\Delta_{P} \\
\operatorname{dim}\left\{x \in \eta_{P}^{-1}\left(\mathscr{O}_{L}\right) \mid \dot{w}^{-1} x \dot{w} \in \eta_{P}^{-1}\left(\mathscr{O}_{L}\right)\right\} \\
\quad \leq 2 \nu_{H}-2 \bar{\nu}+\bar{c}-\operatorname{dim} \mathbb{O}_{\omega}+\Delta_{P},
\end{gathered}
$$


and that (2.2.1) is a strict inequality if $\mathbb{O}_{\omega}$ is bad. Now by (2.1.2), an element $x \in$ $P^{\iota \theta} \cap{ }^{w} P^{\iota \theta}$ can be written as $x=\ell \cdot u=\ell^{\prime} \cdot u^{\prime}$ with $\ell \in L, \ell^{\prime} \in{ }^{w} L, u \in U_{P}^{\iota \theta}, u^{\prime} \in{ }^{w} U_{P}^{\iota \theta}$. Moreover, there exists a unique element $z \in L \cap{ }^{w} L$ such that $\ell=z y^{\prime}, \ell^{\prime}=z y$ with $y^{\prime} \in L \cap{ }^{w} U_{P}, y \in{ }^{w} L \cap U_{P}$. Put

$$
\begin{gathered}
\tilde{Y}=\left\{\left(u, u^{\prime}, y, y^{\prime}, z\right) \in U_{P}^{\iota \theta} \times{ }^{w} U_{P}^{\iota \theta} \times\left({ }^{w} L \cap U_{P}\right) \times\left(L \cap{ }^{w} U_{P}\right) \times\left(L \cap{ }^{w} L\right)\right. \\
\left.\mid y^{\prime} \cdot u=y \cdot u^{\prime}, z y^{\prime} \theta\left(z y^{\prime}\right)^{-1} \in Z_{L}^{\iota \theta} \mathscr{O}_{L}, \dot{w}^{-1} z y \theta(z y)^{-1} \dot{w} \in Z_{L}^{\iota \theta} \mathscr{O}_{L}\right\}
\end{gathered}
$$

and let $Y$ be the quotient of $\widetilde{Y}$ by $E^{\theta}$, where $E=\left({ }^{w} L \cap U_{P}\right) \times\left(L \cap{ }^{w} U_{P}\right) \times\left(L \cap{ }^{w} L\right)$ and $E^{\theta}$ acts on $\tilde{Y}$ by

$$
\left(\ell_{1}, \ell_{2}, z_{1}\right):\left(u, u^{\prime}, y, y^{\prime}, z\right) \mapsto\left(\ell_{2} u \ell_{2}^{-1}, \ell_{1} u^{\prime} \ell_{1}^{-1}, z_{1} y \ell_{1}^{-1}, z_{1} y^{\prime} \ell_{2}^{-1}, z z_{1}^{-1}\right) .
$$

Then (2.2.1) can be rewritten as

$$
\operatorname{dim} Y \leq 2 \nu_{H}-2 \bar{\nu}+\bar{c}+r-\operatorname{dim} \mathbb{O}_{\omega}+\Delta_{P}
$$

where it is the strict inequality if $\mathbb{O}_{\omega}$ is bad. Moreover $(2.2 .2)$ can be rewritten as the inequality obtained by dropping $Z_{L}^{\iota \theta}$ in the definition of $\widetilde{Y}$, and by dropping $r$ from (2.2.3).

We now consider the projection $Y \rightarrow\left({ }^{w} L \cap U_{P}\right) \times\left(L \cap{ }^{w} U_{P}\right) \times\left(L \cap{ }^{w} L\right)$ by $\left(u, u^{\prime}, y, y^{\prime}, z\right) \mapsto\left(y, y^{\prime}, z\right)$. For fixed $y, y^{\prime}, z$, the fibre $Y_{y, y^{\prime}, z}$ can be written as

$$
Y_{y, y^{\prime}, z}=\left\{\left(u, u^{\prime}\right) \in U_{P}^{\iota \theta} \times{ }^{w} U_{P}^{\iota \theta} \mid y^{\prime} \cdot u=y \cdot u^{\prime}\right\}
$$

We note that

$$
\operatorname{dim} Y_{y, y^{\prime}, z}=\operatorname{dim}\left(U_{P} \cap{ }^{w} U_{P}\right)^{\iota \theta} .
$$

In fact, put

$$
\begin{aligned}
\widetilde{u} & =\left(y^{\prime-1} y y^{\prime}\right)^{-1} \cdot u, \\
\widetilde{u}^{\prime} & =y^{\prime-1} \cdot u^{\prime}
\end{aligned}
$$

Since $y \in U_{P}, y^{\prime} \in L$, we have $y^{\prime-1} y y^{\prime} \in U_{P}$. Thus $\widetilde{u} \in U_{P}^{\iota \theta}$. On the other hand, since $y^{\prime} \in{ }^{w} U_{P}$, we have $\widetilde{u}^{\prime} \in{ }^{w} U_{P}^{\iota \theta}$. Thus the variety $Y_{y, y^{\prime}, z}$ is isomorphic to the variety $\left\{\left(\widetilde{u}, \widetilde{u}^{\prime}\right) \in U_{P}^{\iota \theta} \times{ }^{w} U_{P}^{\iota \theta} \mid \widetilde{u}=\widetilde{u}^{\prime}\right\}$. Hence $\operatorname{dim} Y_{y, y^{\prime}, z}=\operatorname{dim}\left(U_{P} \cap{ }^{w} U_{P}\right)^{\iota \theta}$. (2.2.4) holds.

Since the fibres $Y_{y, y^{\prime}, z}$ have constant dimension, (2.2.3) can be rewritten as follows;

$$
\operatorname{dim} D \leq 2 \nu_{H}-2 \bar{\nu}+\bar{c}+r-\operatorname{dim} \mathbb{O}_{\omega}+\Delta_{P}-\operatorname{dim}\left(U_{P} \cap{ }^{w} U_{P}\right)^{\iota \theta},
$$

where

$$
D=\left\{\left(y, y^{\prime}, z\right) \in\left({ }^{w} L \cap U_{P}\right) \times\left(L \cap{ }^{w} U_{P}\right) \times\left(L \cap{ }^{w} L\right)\right.
$$




$$
\left.\mid z y^{\prime} \theta\left(z y^{\prime}\right)^{-1} \in Z_{L}^{\iota \theta} \mathscr{O}_{L}, \dot{w}^{-1} z y \theta(z y)^{-1} \dot{w} \in Z_{L}^{\iota \theta} \mathscr{O}_{L}\right\} / E^{\theta}
$$

and the action of $E^{\theta}$ is defined similarly.

We now compute the difference of $\operatorname{dim}\left(U_{P} \cap{ }^{w} U_{P}\right)^{\theta}$ and $\operatorname{dim}\left(U_{P} \cap{ }^{w} U_{P}\right)^{\iota \theta}$. According to the decomposition in (2.1.3), $U_{P} \cap{ }^{w} U_{P}$ is the direct product of those subgroups corresponding to $G_{0}$ or $G_{i} \times G_{i}(1 \leq i \leq t)$ in (2.1.3). For $G_{i} \times G_{i}$, its $\theta$-fixed part and $\iota \theta$-fixed part are isomorphic. Hence we have only to consider the part $G_{0} \cap P$. We assume that $G_{0} \cap L^{\iota \theta}$ is expressed as in the right hand side of (2.1.1). Put $n=[N / 2], n_{0}=\left[N_{0} / 2\right]$. The Weyl subgroup $W_{0}$ of $W_{H}$ corresponding to $G_{0}$ is isomorphic to $S_{n} \ltimes(\mathbf{Z} / 2 \mathbf{Z})^{n^{\prime}}$, where $n^{\prime}=n$ (resp $n^{\prime}=n-1$ ) if $N$ is odd (resp. even), which we identify with a subgroup of signed permutations of $n$ letters $\{1, \ldots, n\}$. For $w \in W_{H}$, let $w_{0}$ be the element corresponding to $W_{0}$ under the decomposition in (2.1.3). Put

$$
b_{w}=\sharp\left\{i \mid 1 \leq i \leq n-n_{0}, 1 \leq w_{0}^{-1}(i) \leq n-n_{0}\right\} .
$$

Note that the action of $\theta$ on $U_{P} \cap{ }^{w} U_{P}$ can be described from the formula in (1.5.2), and its group version in 1.6, by using (1.10.1) and (1.10.2). In particular, we have

$$
\operatorname{dim}\left(U_{P} \cap{ }^{w} U_{P}\right)^{\theta}=\operatorname{dim}\left(U_{P} \cap{ }^{w} U_{P}\right)^{\iota \theta}-b_{w}
$$

Since $L \cap{ }^{w} P$ is a parabolic subgroup of $L$ with a Levi decomposition $L \cap{ }^{w} P=$ $\left(L \cap{ }^{w} L\right)\left(L \cap{ }^{w} U_{P}\right)$, and similarly for ${ }^{w} L \cap P,{ }^{w} L \cap P=\left({ }^{w} L \cap L\right)\left({ }^{w} L \cap U_{P}\right)$, we have $\operatorname{dim}\left(L \cap{ }^{w} L\right)+\operatorname{dim}\left(L \cap{ }^{w} U_{P}\right)+\operatorname{dim}\left({ }^{w} L \cap U_{P}\right)=\operatorname{dim} L$. Thus

$$
\operatorname{dim}\left(P \cap{ }^{w} P\right)^{\theta}=2 \bar{\nu}+\operatorname{dim} T^{\theta}+\operatorname{dim}\left(U_{P} \cap{ }^{w} U_{P}\right)^{\theta} .
$$

It follows, by (2.2.7), that

$$
\operatorname{dim}\left(U_{P} \cap{ }^{w} U_{P}\right)^{\iota \theta}=2 \nu_{H}-2 \bar{\nu}-\operatorname{dim} \mathbb{O}_{\omega}+b_{w} .
$$

Hence (2.2.5) is equivalent to the form

$$
\operatorname{dim} D \leq \bar{c}+r+\Delta_{P}-b_{w}
$$

Thus in order to prove (2.2.1), we have only to show $(2.2 .9)$, where the strict inequality holds if $\mathbb{O}_{\omega}$ is bad.

A similar discussion shows that, in order to prove (2.2.2), we have only to show

$$
\operatorname{dim} D^{\prime} \leq \bar{c}+\Delta_{P}-b_{w}
$$

where

$$
\begin{aligned}
& D^{\prime}=\left\{\left(y, y^{\prime}, z\right) \in\left({ }^{w} L \cap U_{P}\right) \times\left(L \cap{ }^{w} U_{P}\right) \times\left(L \cap{ }^{w} L\right)\right. \\
& \left.\mid z y^{\prime} \theta\left(z y^{\prime}\right)^{-1} \in \mathscr{O}_{L}, \dot{w}^{-1} z y \theta(z y)^{-1} \dot{w} \in \mathscr{O}_{L}\right\} / E^{\theta} .
\end{aligned}
$$


We consider (2.2.10). Since ${ }^{w} P$ and $L$ contain a common maximal torus $T$, $Q=L \cap^{w} P$ is a $\theta$-stable parabolic subgroup of $L$ with Levi decomposition $Q=M U_{Q}$, where $M=L \cap{ }^{w} L$ and $U_{Q}=L \cap{ }^{w} U_{P}$. Hence by replacing $G, P, L$ by $L, Q, M$, one can define a map $\eta_{Q}: Q^{\iota \theta} \rightarrow M^{\iota \theta}$ as in the case of $\eta_{P}$. Similarly, for a parabolic subgroup $Q^{\prime}={ }^{w} L \cap P=M U_{Q^{\prime}}$ of ${ }^{w} L$ with $U_{Q^{\prime}}={ }^{w} L \cap U_{P}$, the map $\eta_{Q^{\prime}}: Q^{\prime \prime \theta} \rightarrow M^{\iota \theta}$ can be defined. Here $\eta_{Q}\left(z y^{\prime} \theta\left(z y^{\prime}\right)^{-1}\right)=z \theta(z)^{-1}$ for $z \in M, y^{\prime} \in U_{Q}$, and similarly, $\eta_{Q^{\prime}}\left(z y \theta(z y)^{-1}\right)=z \theta(z)^{-1}$. We note that

(2.2.11) There exist finitely many $\left(M^{\theta}\right)^{0}$-orbits $\widehat{\mathscr{O}}_{1}, \ldots, \widehat{\mathscr{O}}_{m}$ in $M^{\iota \theta}$ such that $z \theta(z)^{-1}$ is contained in $\bigcup_{i} \widehat{\mathscr{O}}_{i}$ if $\left(y, y^{\prime}, z\right) \in D^{\prime}$.

In fact, by definition of $D^{\prime}, z \theta(z)^{-1}$ is contained in $\mathscr{O}_{L} U_{Q} \cap M^{\iota \theta}$, hence its semisimple part $z_{1}$ is contained in $\mathscr{O}_{s}$, where $\mathscr{O}_{s}$ is a single $L_{H^{-}}$orbit in $L^{\iota \theta}$ obtained from a semisimple element, say $s \in M^{\iota \theta}$. But $\mathscr{O}_{s} \cap M^{\iota \theta}$ splits into finitely many $\left(M^{\theta}\right)^{0}$-orbits. Here note that $Z_{M}(s)$ is $\theta$-stable, and $Z_{M}(s)^{\iota \theta}$ has only finitely many unipotent $Z_{M}^{0}(s)^{\theta}$-orbits ([R, Proposition 7.4]). (2.2.11) follows from this.

Let $\pi_{3}: D^{\prime} \rightarrow M^{\iota \theta}$ be the map defined by $\left(y, y^{\prime}, z\right) \mapsto \bar{z}=z \theta(z)^{-1}$. By (2.2.11), $\bar{z} \in \bigcup_{i} \widehat{\mathscr{O}}_{i}$, and for each $\bar{z} \in \widehat{\mathscr{O}}_{i}, \pi_{3}^{-1}(\bar{z})$ is isomorphic to the product of the varieties as in (i), namely, $\left(\mathscr{O}_{L} \cap \eta_{Q}^{-1}(\bar{z})\right) \times\left(\mathscr{O}_{w_{L}} \cap \eta_{Q^{\prime}}^{-1}(\bar{z})\right)$. Hence by induction hypothesis, we have

$$
\operatorname{dim} \pi_{3}^{-1}(\bar{z}) \leq \frac{1}{2}\left(\bar{c}-\operatorname{dim} \widehat{\mathscr{O}}_{i}\right)+\frac{1}{2}\left(\bar{c}-\operatorname{dim} \widehat{\mathscr{O}}_{i}\right)+\Delta_{Q}
$$

(Note that $\left.\Delta_{Q}=\Delta_{Q^{\prime}}\right)$. It follows that $\operatorname{dim} \pi_{3}^{-1}\left(\overline{\mathscr{O}}_{i}\right) \leq \bar{c}+\Delta_{Q}$. Since this is true for any $i$, we have $\operatorname{dim} D^{\prime} \leq \bar{c}+\Delta_{Q}$. In order to show (2.2.10), it is enough to see that

$$
\Delta_{Q} \leq \Delta_{P}-b_{w}
$$

We can express $\Delta_{Q}$ as

$$
\Delta_{Q}=\sharp\left\{i \mid n-n_{0}+1 \leq i \leq n, 1 \leq w_{0}^{-1}(i) \leq n-n_{0}\right\} .
$$

By comparing this with (2.2.6), we obtain (2.2.12). Thus (2.2.10) is proved.

Next consider (2.2.9). A similar argument as in the proof of (2.2.10) shows that there exist finitely many orbits $\widehat{\mathscr{O}}_{1}^{\prime}, \ldots, \widehat{\mathscr{O}}_{m^{\prime}}^{\prime}, \widehat{\mathscr{O}}_{1}^{\prime \prime}, \ldots, \widehat{\mathscr{O}}_{m^{\prime \prime}}^{\prime \prime}$ in $\left(M^{\imath \theta}\right)^{0}$ such that $z \theta(z)^{-1}$ is contained in the intersection of $Z_{L}^{\iota \theta}\left(\widehat{\mathscr{O}}_{1}^{\prime} \cup \cdots \cup \widehat{\mathscr{O}}_{m^{\prime}}^{\prime}\right)$ and $Z_{w_{L}}^{\iota \theta}\left(\widehat{\mathscr{O}}_{1}^{\prime \prime} \cup \cdots \cup\right.$ $\left.\widehat{\mathscr{O}}_{m^{\prime \prime}}^{\prime \prime}\right)$. Since $Z_{L}$ and $Z_{w_{L}}$ are contained in the center of $M, z \theta(z)^{-1}$ is contained in $\left(Z_{L} \cap Z_{w_{L}}\right)^{\iota \theta}\left(\widehat{\mathscr{O}}_{1} \cup \cdots \cup \widehat{\mathscr{O}}_{m}\right)$ for some $\left(M^{\theta}\right)^{0}$-orbits $\widehat{\mathscr{O}}_{1}, \ldots, \widehat{\mathscr{O}}_{m}$ in $M^{\iota \theta}$. Thus as in the proof of (2.2.10), we have $\operatorname{dim} D^{\prime} \leq \bar{c}+\operatorname{dim}\left(Z_{L} \cap Z_{w_{L}}\right)^{\iota \theta}+\Delta_{Q}$. Here $\operatorname{dim}\left(Z_{L} \cap Z_{w_{L}}\right)^{\iota \theta} \leq r$, and the strict inequality holds if $\mathbb{O}_{\omega}$ is bad. (2.2.9) follows from this by using (2.2.12). Hence we have proved (iii) and (iv), assuming the induction hypothesis.

Next we show (ii). Put $Z_{\mathscr{O}}^{\prime}=\left\{\left(x, g P_{H}, g^{\prime} P_{H}\right) \in Z^{\prime} \mid x \in \mathscr{O}\right\}$. If $Z_{\mathscr{O}}^{\prime}$ is empty, then the variety in (ii) is also empty, and the inequality holds. So we assume that $Z_{\mathscr{O}}^{\prime}$ is non-empty. From (iv), we have $\operatorname{dim} Z_{\mathscr{O}}^{\prime} \leq d^{\prime}+\Delta_{P}$. Consider the projection $p: Z_{\mathscr{O}}^{\prime} \rightarrow \mathscr{O}$ to the first factor. Then each fibre is isomoprhic to the product of two 
copies of the variety in (ii). Thus we have

$$
\begin{aligned}
\operatorname{dim}\left\{g P_{H} \in H / P_{H} \mid g^{-1} x g \in \eta_{P}^{-1}\left(\mathscr{O}_{L}\right)\right\} & =\left(\operatorname{dim} Z_{\mathscr{O}}^{\prime}-\operatorname{dim} \mathscr{O}\right) / 2 \\
& \leq\left(d^{\prime}+\Delta_{P}-c\right) / 2 \\
& =\nu_{H}-\bar{\nu}+\left(\bar{c}-c+\Delta_{P}\right) / 2
\end{aligned}
$$

Hence (ii) holds.

Finally we show (i). Consider the variety $R=\left\{\left(x, g P_{H}\right) \in \mathscr{O} \times H / P_{H} \mid x \in\right.$ $\left.\eta_{P}^{-1}\left(\mathscr{O}_{L}\right)\right\}$. By projecting to the first factor, and by using (ii), we see that $\operatorname{dim} R \leq$ $\nu_{H}-\bar{\nu}+(c+\bar{c}) / 2+\Delta_{P} / 2$. If we project to the second factor, each fibre is isomorphic to the variety $\mathscr{O} \cap \eta_{P}^{-1}\left(\mathscr{O}_{L}\right)$. Hence

$$
\begin{aligned}
\operatorname{dim}\left(\mathscr{O} \cap \eta_{P}^{-1}\left(\mathscr{O}_{L}\right)\right) & \leq \nu_{H}-\bar{\nu}+(c+\bar{c}) / 2+\Delta_{P} / 2-\operatorname{dim} H / P_{H} \\
& =(c+\bar{c}) / 2+\Delta_{P} / 2
\end{aligned}
$$

Now we consider the map $\mathscr{O} \cap \eta_{P}^{-1}\left(\mathscr{O}_{L}\right) \rightarrow \mathscr{O}_{L}$ by $x \mapsto \eta_{P}(x)$. Then each fibre is isomorphic to the variety considered in (i). Hence the dimension of this variety is $\leq(c+\bar{c}) / 2+\Delta_{P} / 2-\bar{c}=(c-\bar{c}) / 2+\Delta_{P} / 2$. This proves (i). The proposition is proved.

2.3. We keep the setting in 2.1. Let $G_{\text {uni }}$ be the set of unipotent elements in $G$, and put $G_{\text {uni }}^{\iota \theta}=G^{\iota \theta} \cap G_{\text {uni }}$. By [R, proposition 7.4], $G_{\text {uni }}^{\iota \theta}$ has finitely many $H$-orbits. We define $L_{\text {uni }}^{\iota \theta}$ for $L$ similarly to $G$. Let $\mathscr{O} \subset G_{\text {uni }}^{\iota \theta}, \mathscr{O}_{L} \subset L_{\text {uni }}^{\iota \theta}$. Take $u \in \mathscr{O}, v \in \mathscr{O}_{L}$, and fix them. We define varieties

$$
\begin{aligned}
& Y_{u, v}=\left\{g Z_{L_{H}}^{0}(v) U_{P}^{\theta} \mid g \in H, g^{-1} u g \in \eta_{P}^{-1}(v)\right\} \\
& \widetilde{Y}_{u, v}=\left\{g \in H \mid g^{-1} u g \in \eta_{P}^{-1}(v)\right\}
\end{aligned}
$$

Note that $\eta_{P}^{-1}(v)=\left(v U_{P}\right)^{\iota \theta}$, hence $Y_{u, v}$ is well-defined. $Z_{H}(u) \times Z_{L_{H}}(v) U_{P}^{\theta}$ acts on $\widetilde{Y}_{u, v}$ by $(x, y): g \mapsto x g y^{-1}\left(x \in Z_{H}(u), y \in Z_{L_{H}}(v) U_{P}^{\theta}, g \in \widetilde{Y}_{u, v}\right)$. Let $\phi: \widetilde{Y}_{u, v} \rightarrow$ $Y_{u, v} \simeq \widetilde{Y}_{u, v} / Z_{L_{H}}^{0}(v) U_{P}^{\theta}, \varphi: \widetilde{Y}_{u, v} \rightarrow Z_{H}^{0}(u) \backslash \widetilde{Y}_{u, v}$ be the quotient maps. We define $\xi: \widetilde{\mathscr{O}}=H / Z_{H}^{0}(u) \rightarrow H / Z_{H}(u) \simeq \mathscr{O}$ by $g Z_{H}^{0}(u) \mapsto g u g^{-1}$. Then $\xi$ is a finite Galois covering with Galois group $A_{H}(u)=Z_{H}(u) / Z_{H}^{0}(u)$. We have the following commutative diagram.

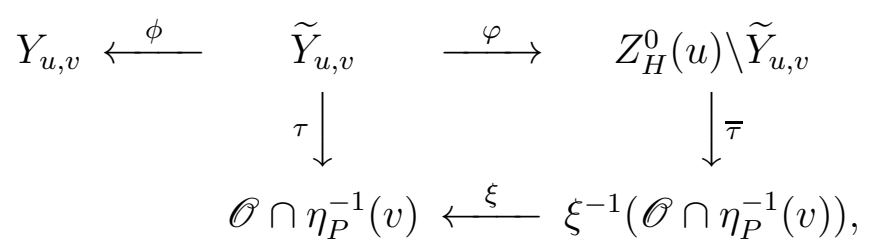


where $\tau: g \mapsto g^{-1} u g, \bar{\tau}: Z_{H}^{0}(u) g \mapsto g^{-1} Z_{H}^{0}(u)$. Note that $\bar{\tau}$ gives an isomorphism $Z_{H}^{0}(u) \backslash \widetilde{Y}_{u, v} \stackrel{\sim}{\rightarrow} \xi^{-1}\left(\mathscr{O} \cap \eta_{P}^{-1}(v)\right)$. Put

$$
\begin{aligned}
& \delta=\left(\operatorname{dim} \mathscr{O}-\operatorname{dim} \mathscr{O}_{L}\right) / 2+\Delta_{P} / 2, \\
& s=\left(\operatorname{dim} Z_{H}(u)-\operatorname{dim} Z_{L_{H}}(v)\right) / 2+\Delta_{P} / 2 .
\end{aligned}
$$

By (2.3.1), we have $\operatorname{dim}\left(\mathscr{O} \cap \eta_{P}^{-1}(v)\right)=\operatorname{dim} \xi^{-1}\left(\mathscr{O} \cap \eta_{P}^{-1}(v)\right)=\operatorname{dim} Z_{H}^{0}(u) \backslash \tilde{Y}_{u, v}$. Hence if we put $d_{u, v}=\operatorname{dim}\left(\mathscr{O} \cap \eta_{P}^{-1}(v)\right)$, we have

$$
\begin{aligned}
\operatorname{dim} \widetilde{Y}_{u, v} & =d_{u, v}+\operatorname{dim} Z_{H}(u), \\
\operatorname{dim} Y_{u, v} & =d_{u, v}+\operatorname{dim} Z_{H}(u)-\operatorname{dim} Z_{L_{H}}(v)-\operatorname{dim} U_{P}^{\theta} .
\end{aligned}
$$

On the other hand, by Proposition 2.2 (i), we have $d_{u, v} \leq \delta$. It follows that $\operatorname{dim} Y_{u, v} \leq s$, and that the equality holds if and only if $d_{u, v}=\delta$. Let $I\left(Y_{u, v}\right)$ be the set of irreducible components of $Y_{u, v}$ of dimension $s$. Similarly, let $I\left(\widetilde{Y}_{u, v}\right)$ (resp. $\left.I_{u, v}\right)$ be the set of irreducible components of $\widetilde{Y}_{u, v}\left(\operatorname{resp} . \xi^{-1}\left(\mathscr{O} \cap \eta_{P}^{-1}(v)\right)\right)$ of dimension $\delta+\operatorname{dim} Z_{H}(u)$ (resp. dimension $\delta$ ). By (2.3.1), we have a natural bijection $I\left(Y_{u, v}\right) \simeq I\left(\widetilde{Y}_{u, v}\right) \simeq I_{u, v}$.

Put $A_{H}(u)=Z_{H}(u) / Z_{H}^{0}(u)$. Since $Z_{H}(u)$ acts on $Y_{u, v}$ from the left, $A_{H}(u)$ acts on $I\left(Y_{u, v}\right)$ as permutations of irreducible components. Similarly, $A_{H}(u)$ acts on $I\left(\widetilde{Y}_{u, v}\right), I_{u, v}$, and the above bijection turns out to be $A_{H}(u)$-equivariant.

Definition 2.4. Let $\mathscr{O}$ be an $H$-orbit in $G_{\text {uni }}^{\iota \theta}$, and take $u \in \mathscr{O}$. $\tau \in A_{H}(u)^{\wedge}$ is said to be cuspidal if $\tau$ does not appear in the permutation representation of $A_{H}(u)$ on $I\left(Y_{u, v}\right)$ for any $\theta$-stable Levi subgroup $L$ of any $\theta$-stable proper parabolic subgroup $P$ of $G$, and for any $v \in L_{\text {uni }}^{\iota \theta}$. An $H$-equivariant simple local system $\mathscr{E}$ on $\mathscr{O}$ corresponding to $\tau \in A_{H}(u)^{\wedge}$ is said to be cuspidal if $\tau$ is cuspidal.

It follows from the definition that if $\tau$ is cuspidal, then its dual representation $\tau^{*}$ is also cuspidal.

Lemma 2.5. The local system $\mathscr{E}$ on $\mathscr{O}$ is cuspidal if and only if for any proper $\theta$-stable parabolic subgroup $P$ of $G$, and for any $v \in L_{\text {uni }}^{\iota \theta}$, we have

$$
H_{c}^{2 \delta}\left(\mathscr{O} \cap \eta_{P}^{-1}(v), \mathscr{E}\right)=0
$$

Proof. Let $\xi: \widetilde{\mathscr{O}} \rightarrow \mathscr{O}$ be the finite Galois covering with Galois group $A_{H}(u)$. Then $\xi_{*} \overline{\mathrm{Q}}_{l}$ is a semisimple local system on $\mathscr{O}$ equipped with $A_{H}(u)$-action, and we have $\mathscr{E}=\operatorname{Hom}_{A_{H}(u)}\left(\tau, \xi_{*} \overline{\mathbf{Q}}_{l}\right)$. Hence

$$
\begin{aligned}
H_{c}^{i}\left(\mathscr{O} \cap \eta_{P}^{-1}(v), \mathscr{E}\right) & \simeq\left(H_{c}^{i}\left(\mathscr{O} \cap \eta_{P}^{-1}(v), \xi_{*} \overline{\mathbf{Q}}_{l}\right) \otimes \tau^{*}\right)^{A_{H}(u)} \\
& \simeq\left(H_{c}^{i}\left(\xi^{-1}\left(\mathscr{O} \cap \eta_{P}^{-1}(v)\right), \overline{\mathbf{Q}}_{l}\right) \otimes \tau^{*}\right)^{A_{H}(u)}
\end{aligned}
$$

where $\tau^{*}$ is the dual representation of $\tau$. This implies that the condition $\tau$ does not appear in the permutation representation of $I_{u, v}$ is equivalent to the condition 
$H_{c}^{2 \delta}\left(\mathscr{O} \cap \eta_{P}^{-1}(v), \mathscr{E}\right)=0$. Since $I_{u, v} \simeq I\left(Y_{u, v}\right)$ with $A_{H}(u)$-action, the lemma follows.

2.6. More generally, we consider $G$ and an involution $\theta: G \rightarrow G$ as in (2.1.3). The definition of cuspidal local system $\mathscr{E}$ on $\mathscr{O}$ can be generalized to this case, and Lemma 2.5 holds. Let $\mathscr{E}$ be a local system on $\mathscr{O}$. Then, under the isomorphism in $(2.1 .4), \mathscr{O} \simeq \mathscr{O}_{0} \times \mathscr{O}_{1} \times \cdots \times \mathscr{O}_{t}$, where $\mathscr{O}_{0} \subset G_{0}^{\iota \theta}$ and $\mathscr{O}_{i} \subset\left(G_{i} \times G_{i}\right)^{\iota \theta} \simeq G_{i}$ for $i=1, \ldots, t$. Thus $\mathscr{E}$ can be written as $\mathscr{E}=\mathscr{E}_{0} \otimes \mathscr{E}_{1} \otimes \cdots \otimes \mathscr{E}_{t}$, where $\mathscr{E}_{i}$ is a local system on $\mathscr{O}_{i}$ for each $i$. By Lemma 2.5, it is easy to see that $\mathscr{E}$ is cuspidal on $\mathscr{O}$ if and only if $\mathscr{E}_{i}$ is cuspidal on $\mathscr{O}_{i}$ for each $i$. But note that the definition of cuspidality for $\mathscr{E}_{i}(i \geq 1)$ is exactly the same as the definition of cuspidality in the case of reductive groups in [L1, 2.4]. It is well-known that in the case of $G L_{n}$, there does not exist a cuspidal local system unless $n=1$. Hence we have the following.

Lemma 2.7. Assume that $H=S O_{N}$. Let $L$ be a $\theta$-stable Levi subgroup of a $\theta$-stable parabolic subgroup of $G$. Let $\mathscr{O}_{L}$ be an $L_{H}$-orbit in $L_{\text {uni }}^{\iota \theta}$. If there exists a cuspidal local system on $\mathscr{O}_{L}$, then $L_{H} \simeq\left(G L_{1}\right)^{a} \times S O_{N_{0}}$, where $a=\left(N-N_{0}\right) / 2$.

Remark 2.8. In the case where $H=S p_{N}$, Proposition 2.2 still holds by putting $\Delta_{P}=0$.

\section{Admissible complexes}

3.1. We follow the setting in 1.7, and consider $H=\left(G^{\theta}\right)^{0} \simeq S O_{N}$. Let us fix a $\theta$-stable Borel subgroup $B$ and a $\theta$-stable maximal torus $T$ contained in $B$. By Lemma 1.2, the pair $(B, T)$ is $G^{\theta}$-conjugate to the specific choice of the Borel subgroup and the maximal torus given in 1.8. Let $U$ be the unipotent radical of $B$. We define a subgroup $\mathscr{D}$ of $U$ by $\mathscr{D}=U\left(\Delta_{0}\right)$. $\mathscr{D}$ is conjugate under $G^{\theta}$ to the corresponding group defined in 1.10. In particular, we have $\mathscr{D}=U\left(\Delta_{0}\right)^{\iota \theta}$.

Let $P=L U_{P}$ be a $\theta$-stable parabolic subgroup of $G$ containing $B$ such that $T \subset L$. Here we assume that $L^{\theta} \simeq\left(G L_{1}\right)^{a} \times G L_{N_{0}}^{\theta}$ with $N_{0}=N-2 a$ for $0 \leq a \leq n$ (see Lemma 2.7). Let $\mathscr{O}_{L}$ be an $L_{H^{-o r b i t}}$ in $L_{\text {uni }}^{\iota \theta}$, and consider $\Sigma=Z_{L}^{\iota \theta} \times \mathscr{O}_{L} \subset L^{\iota \theta}$. Note that $Z_{L}^{\iota \theta} \simeq\left(G L_{1}\right)^{a}$. Let $\left(Z_{L}\right)_{\mathrm{reg}}^{\iota \theta}$ be the set of $\left(t_{1}, \ldots, t_{a}\right) \in\left(G L_{1}\right)^{a}$ such that $t_{i}$ are all distinct, under the above isomorphism. Put $\Sigma_{\text {reg }}=\left(Z_{L}\right)_{\text {reg }}^{\iota \theta} \times \mathscr{O}_{L}$. Then $\Sigma_{\text {reg }}$ is open dense in $\Sigma$. Put $\mathscr{D}_{P}=\mathscr{D} \cap U_{P}$. Then $\mathscr{D}_{P}$ is a closed subgroup of $U_{P}$ contained in $U_{P}^{\iota \theta}$ such that $\mathscr{D}_{P} \simeq \mathbf{k}^{a}$. Moreover by $1.11, \mathscr{D}_{P}$ is stable under the conjugation action of $L_{H}$ on $U_{P}^{\iota \theta}$. By (1.11.1), any element in $\Sigma$ commutes with any element in $\mathscr{D}_{P}$. Hence

$$
\Sigma \mathscr{D}_{P} \subset P^{\iota \theta}
$$

Note that $\operatorname{dim} \mathscr{D}_{P}=a=\Delta_{P}(\operatorname{see}(2.1 .6))$. 
Let $\mathscr{D}_{P}^{0}$ be the subset of $\mathscr{D}_{P} \simeq \mathbf{k}^{a}$ consisting of $\boldsymbol{\xi}=\left(\xi_{1}, \ldots, \xi_{a}\right) \in \mathbf{k}^{a}$ such that $\xi_{i} \neq 0$ for any $i$. We define varieties

$$
\begin{aligned}
& \widetilde{Y}_{(L, \Sigma)}=\left\{\left(x, g L_{H}\right) \in G^{\iota \theta} \times H / L_{H} \mid g^{-1} x g \in \Sigma_{\operatorname{reg}} \mathscr{D}_{P}^{0}\right\} \\
& Y_{(L, \Sigma)}=\bigcup_{g \in H} g\left(\Sigma_{\operatorname{reg}} \mathscr{D}_{P}^{0}\right) g^{-1}
\end{aligned}
$$

We show a lemma.

Lemma 3.2. $\quad$ (i) $Y_{(L, \Sigma)}$ is a smooth, irreducible variety with

$$
\operatorname{dim} Y_{(L, \Sigma)}=2 \nu_{H}-2 \nu_{L_{H}}+\operatorname{dim} \Sigma+\Delta_{P}
$$

(ii) Let $P^{\prime}=L^{\prime} U_{P^{\prime}}$ be another $\theta$-stable parabolic subgroup with $\theta$-stable Levi subgroup $L^{\prime},\left(L^{\prime}, \Sigma^{\prime}\right)$ a pair defined for $L^{\prime}$, similarly to $(L, \Sigma)$. Assume that $\left(L^{\prime}, \Sigma^{\prime}\right)$ is not $H$-conjugate to $(L, \Sigma)$. Then we have $Y_{(L, \Sigma)} \cap Y_{\left(L^{\prime}, \Sigma^{\prime}\right)}=\emptyset$.

Proof. For each $g \in G^{\iota \theta}$, let $g_{s}$ be the semisimple part of $g$ in $G^{\iota \theta}$, and $Z_{H}\left(g_{s}\right)$ be the stabilizer of $g_{s}$ in $H$. Put $Z_{g}=Z_{Z_{H}\left(g_{s}\right)}^{0}$, the connected center of $Z_{H}\left(g_{s}\right)$. Then $H(g)=Z_{H}\left(Z_{g}\right)$ is a Levi subgroup of some parabolic subgroup of $H$. If $g \in Y_{(L, \Sigma)}, H(g)$ is a Levi subgroup conjugate to $L_{H}$ under $H$. By identifying $H / N_{H}\left(L_{H}\right)$ with the set of Levi subgroups of $H$ conjugate to $L_{H}$, we define a map $\zeta: Y_{(L, \Sigma)} \rightarrow H / N_{H}\left(L_{H}\right)$ by $g \mapsto H(g)$. Then one can show that

$$
\zeta: Y_{(L, \Sigma)} \simeq H \times{ }^{N_{H}\left(L_{H}\right)} \zeta^{-1}\left(L_{H}\right) \rightarrow H / N_{H}\left(L_{H}\right)
$$

is a locally trivial fibration with fibre isomorphic to $\zeta^{-1}\left(L_{H}\right)$. We have

$$
\zeta^{-1}\left(L_{H}\right)=\bigcup_{w \in N_{H}\left(L_{H}\right) / L_{H}} w\left(\Sigma_{\mathrm{reg}} \mathscr{D}_{P}^{0}\right) w^{-1}
$$

Here $N_{H}\left(L_{H}\right) / L_{H} \simeq S_{a} \ltimes(\mathbf{Z} / 2 \mathbf{Z})^{a^{\prime}}$, where $S_{a}$ is the symmetric group of degree $a$, and $a^{\prime}=a$ unless $a=n$ and $N$ is even, in which case $a^{\prime}=n-1 . w \in N_{H}\left(L_{H}\right) / L_{H}$ leaves $\Sigma_{\text {reg }}$ stable, and $\sigma \in S_{a}$ leaves $\mathscr{D}_{P}^{0}$ stable. Hence

$$
\xi^{-1}\left(L_{H}\right) \simeq \Sigma_{\text {reg }} \times \bigcup_{w \in(\mathbf{Z} / 2 \mathbf{Z})^{a^{\prime}}} w \mathscr{D}_{P}^{0} w^{-1}
$$

Since $w \mathscr{D}_{P}^{0} w^{-1}$ are mutually disjoint for $w \in(\mathbf{Z} / 2 \mathbf{Z})^{a^{\prime}}$, we see that $\zeta^{-1}\left(L_{H}\right)$ is a disjoint union of smooth pieces $\Sigma_{\text {reg }} \times w \mathscr{D}_{P}^{0} w^{-1}$. Hence $\zeta^{-1}\left(L_{H}\right)$ is smooth, and so $Y_{(L, \Sigma)}$ is smooth by (3.2.2). Since $\Sigma_{\text {reg }} \mathscr{D}_{P}^{0}$ is irreducible, $Y_{(L, \Sigma)}$ is also irreducible. The dimension formula (3.2.1) follows from (3.2.2) and (3.2.3). Thus (i) holds. (ii) is immediate since for $g \in Y_{(L, \Sigma)}, H(g)$ determines a unique Levi subgroup in $H$, and once $L_{H}=H(g)$ is given, $g$ determines a unique $L_{H}$-orbit $\mathscr{O}_{L}$, hence determines $\Sigma$ uniquely. The lemma is proved. 
Remark 3.3 The definition of $Y_{(L, \Sigma)}$ given here depends on the special choice of $P$ as in 3.1. The discussion in 3.1 can not be applied for arbitrary $L$. So, we cannot discuss the partition of $G^{\iota \theta}$ in terms of various $Y_{(L, \Sigma)}$ as given in [L1, 3.1].

3.4. By fixing $(L, \Sigma)$, put $Y=Y_{(L, \Sigma)}, \widetilde{Y}=\widetilde{Y}_{(L, \Sigma)}$. Recall the map $\eta_{P}: P^{\iota \theta} \rightarrow$ $L^{\iota \theta}$. Let $\bar{\Sigma}$ be the closure of $\Sigma$ in $L^{i \theta}$. We define varieties $X, \widetilde{X}$ by

$$
\begin{aligned}
& \widetilde{X}=\left\{\left(x, g P_{H}\right) \in G^{\iota \theta} \times H / P_{H} \mid g^{-1} x g \in \eta_{P}^{-1}(\bar{\Sigma})\right\}, \\
& X=\bigcup_{g \in H} g\left(\eta_{P}^{-1}(\bar{\Sigma})\right) g^{-1}
\end{aligned}
$$

and a map $\pi: \widetilde{X} \rightarrow X$ by $\left(x, g P_{H}\right) \mapsto x$. Then $\pi$ is proper and surjective. We have

$$
\widetilde{X} \simeq H \times{ }^{P_{H}} \eta_{P}^{-1}(\bar{\Sigma}) .
$$

We have a lemma.

Lemma 3.5. $\quad$ (i) $X$ is a closed irreducible subvariety of $G^{\iota \theta}$ such that the closure $\bar{Y}$ of $Y$ coincides with $X$. We have $\operatorname{dim} \widetilde{X}=\operatorname{dim} X$.

(ii) The map $\left(x, g L_{H}\right) \mapsto\left(x, g P_{H}\right)$ gives an isomorphism $\gamma: \widetilde{Y} \underset{\rightarrow}{\longrightarrow} \pi^{-1}(Y)$.

(iii) $Y$ is an open dense subset of $X$. Hence $Y$ is an irreducible, locally closed smooth subvariety of $G^{\iota \theta}$.

Proof. (i) Since $\eta_{P}$ is an affine space bundle with fibre isomorphic to $U_{P}^{\iota \theta}$ by (2.1.2), we have $\operatorname{dim} \eta_{P}^{-1}(\bar{\Sigma})=\operatorname{dim} \Sigma+\operatorname{dim} U_{P}^{\iota \theta}$. Thus (3.4.1) shows that $\widetilde{X}$ is irreducible with

$$
\begin{aligned}
\operatorname{dim} \widetilde{X} & =\operatorname{dim} H-\operatorname{dim} P_{H}+\pi_{P}^{-1}(\bar{\Sigma}) \\
& =\operatorname{dim} H-\operatorname{dim} P_{H}+\operatorname{dim} \Sigma+\operatorname{dim} U_{P}^{\iota \theta} \\
& =2 \nu_{H}-2 \nu_{L_{H}}+\operatorname{dim} \Sigma-\operatorname{dim} U_{P}^{\theta}+\operatorname{dim} U_{P}^{\iota \theta} .
\end{aligned}
$$

By (1.10.1) and (1.10.2), we have $\operatorname{dim} U_{P}^{\iota \theta}=\operatorname{dim} U_{P}^{\theta}+\Delta_{P}$. Hence by comparing the last equality with (3.2.1), we have

$$
\operatorname{dim} \widetilde{X}=\operatorname{dim} Y .
$$

Since $\widetilde{X}$ is irreducible and $\pi$ is proper, $X$ is an irreducible closed subset of $G^{\iota \theta}$. Since $Y \subset X$, we have $\bar{Y} \subset X$. We have

$$
\operatorname{dim} Y \leq \operatorname{dim} X \leq \operatorname{dim} \widetilde{X}=\operatorname{dim} Y
$$

by (3.5.1). Hence $\operatorname{dim} Y=\operatorname{dim} X=\operatorname{dim} \tilde{X}$. Since $X$ and $\bar{Y}$ are irreducible, closed with same dimension, we have $X=\bar{Y}$. This proves (i).

(ii) The map $\left(x, g L_{H}\right) \mapsto\left(x, g P_{H}\right)$ gives a well-defined map $\gamma: \widetilde{Y} \rightarrow \pi^{-1}(Y)$. For $u \in U_{P}, t \in\left(Z_{L}\right)_{\mathrm{reg}}$, if $t u=u t$, then $u \in \mathscr{D}_{P}$. Thus for $1 \neq u \in U_{P}^{\theta}, t \in$ 
$\left(Z_{L}\right)_{\mathrm{reg}}^{\iota \theta}, t^{-1} u^{-1} t u$ produces an element in $U_{P}-\mathscr{D}_{P}$. The injectivity of $\gamma$ follows from this. We will show that $\gamma(\widetilde{Y})=\pi^{-1}(Y)$. Take an element in $\pi^{-1}(x)$ for $x \in \Sigma_{\text {reg }} \mathscr{D}_{P}^{0}$, which is of the form $\left(x, g P_{H}\right)$ with $g^{-1} x g \in \eta_{P}^{-1}(\bar{\Sigma})$. Let $x_{s}$ be the semisimple part of $x$. Then $x_{s} \in\left(Z_{L}\right)_{\mathrm{reg}}^{\iota \theta}$, and $Z_{H}^{0}\left(x_{s}\right)=L_{H}$. On the other hand, since $\bar{\Sigma}=Z_{L}^{\iota \theta} \overline{\mathscr{O}}_{L}, g^{-1} x_{s} g \in Z_{L}^{\iota \theta}$. In particular, $Z_{H}\left(g^{-1} x_{s} g\right) \supset L_{H}$. But since $Z_{H}\left(g^{-1} x_{s} g\right)=g^{-1} L_{H} g$, we have $g^{-1} L_{H} g=L_{H}$, namely, $g \in N_{H}\left(L_{H}\right)$. Here note that $N_{H}\left(L_{H}\right) / L_{H} \simeq S_{a} \ltimes(\mathbf{Z} / 2 \mathbf{Z})^{a^{\prime}}$. Thus $g \in N_{H}\left(L_{H}\right)$ leaves $\Sigma_{\text {reg invariant. By our }}$ assumption $g^{-1} x g \in \bar{\Sigma} U_{P}, g \in N_{H}\left(L_{H}\right)$ should be contained in the inverse image of $S_{a}$ under the map $N_{H}\left(L_{H}\right) \rightarrow N_{H}\left(L_{H}\right) / L_{H}$. Such a $g$ leaves $\mathscr{D}_{P}^{0}$ invariant also. Hence $\left(x, g L_{H}\right) \in \widetilde{Y}$, and we have $\left(x, g P_{H}\right)=\gamma\left(\left(x, g L_{H}\right)\right)$. Since any element in $\pi^{-1}(Y)$ is $H$-conjugate to the element discussed above, we see that $\gamma$ is surjective. Hence $\gamma$ is a bijection. The inverse morphism $\gamma^{-1}: \pi^{-1}(Y) \rightarrow \widetilde{Y}$ is constructed from the above discussion. (ii) is proved.

(iii) Since $X$ is irreducible, it is enough to show that $Y$ is an open subset of $X$. Other properties are already shown in Lemma 3.2. Put $\left(Z_{L}\right)_{1}^{\iota \theta}=Z_{L}^{\iota \theta}-\left(Z_{L}\right)_{\text {reg }}^{\iota \theta}$ and $\Sigma_{1}=\left(Z_{L}\right)_{1}^{\iota \theta} \overline{\mathscr{O}}_{L}$. Then $\Sigma_{1}$ is a closed subset of $\bar{\Sigma}$, and $X_{1}=\bigcup_{g \in H} g\left(\eta_{P}^{-1}\left(\Sigma_{1}\right)\right) g^{-1}$ is a closed subset of $X$. Thus $X-X_{1}$ is an open subset of $X$, and in fact, it coincides with the subset of $X$ consisting of $x$ such that the semisimple part of $x$ is $H$-conjugate to an element in $\left(Z_{L}\right)_{\text {reg }}^{\iota \theta}$. Let $\Sigma_{2}=Z_{L}^{\iota \theta}\left(\overline{\mathscr{O}}_{L}-\mathscr{O}_{L}\right)$. Then $\Sigma_{2}$ is a closed subset of $\bar{\Sigma}$, and $X_{2}=\bigcup_{g \in H} g\left(\eta_{P}^{-1}\left(\Sigma_{2}\right)\right) g$ is a closed subset of $X$. Since $Y=X-\left(X_{1} \cup X_{2}\right), Y$ is open in $X$. (iii) is proved.

3.6. We consider the diagram

$$
\Sigma \stackrel{\alpha_{0}}{\longleftarrow} \widehat{Y} \stackrel{\psi_{0}}{\longrightarrow} \tilde{Y} \stackrel{\pi_{0}}{\longrightarrow} Y
$$

where

$$
\widehat{Y}=\left\{(x, g) \in G^{\iota \theta} \times H \mid g^{-1} x g \in \Sigma_{\text {reg }} \mathscr{D}_{P}^{0}\right\},
$$

and maps are defined by $\pi_{0}\left(x, g L_{H}\right)=x, \psi_{0}(x, g)=\left(x, g L_{H}\right), \alpha_{0}(x, g)=\eta_{P}\left(g^{-1} x g\right)$. Here $H$ acts on $\tilde{Y}$ by $h:\left(x, g L_{H}\right) \mapsto\left(h x h^{-1}, h g L_{H}\right)$, and $\pi_{0}$ is $H$-equivariant with respect to the conjugation action of $H$ on $Y$. On the other hand, $H \times L_{H}$ acts on $\widehat{Y}$ by $(h, \ell):(x, g) \mapsto\left(h x h^{-1}, h g \ell^{-1}\right)$, and $\psi_{0}$ is $H$-equivariant. $H \times L_{H}$ also acts on $\Sigma$ by $(h, \ell): y \mapsto \ell y \ell^{-1}$, and $\alpha_{0}$ is $H \times L_{H^{-}}$equivariant.

Let $\mathscr{E}_{1}$ be an $L_{H}$-equivariant simple local system on $\Sigma$. Since $\Sigma=Z_{L}^{\iota \theta} \times \mathscr{O}_{L}, \mathscr{E}_{1}$ can be written as $\mathscr{E}_{1}=\mathscr{S} \otimes \mathscr{E}_{1}^{\dagger}$, where $\mathscr{S}$ (resp. $\mathscr{E}_{1}^{\dagger}$ ) is a simple local system on $Z_{L}^{\iota \theta}$ (resp. $\left.\mathscr{O}_{L}\right)$. We say that $\left(\Sigma, \mathscr{E}_{1}\right)$ is a cuspidal pair if $\mathscr{E}_{1}^{\dagger}$ is a cuspidal local system on $\mathscr{O}_{L}$. Note that this definition is weaker than the definition of the cuspidal pair in $[\mathrm{L} 1,2.4]$. By abuse of the notation, we also say that $\left(\mathscr{O}_{L}, \mathscr{E}_{1}^{\dagger}\right)$ is a cuspidal pair if $\mathscr{E}_{1}^{\dagger}$ is cuspidal on $\mathscr{O}_{L}$. Now consider an arbitrary $\mathscr{E}_{1}$. Then $\mathscr{E}_{1}$ is $\left(H \times L_{H}\right)$-equivariant with respect to the trivial action of $H$ on $\Sigma$, and $\alpha_{0}^{*} \mathscr{E}_{1}$ is an $\left(H \times L_{H}\right)$-equivariant local system on $\widehat{Y}$. Since $\psi_{0}$ is a principal bundle over $\widetilde{Y}$ with respect to the free action of $L_{H}$, there exists a unique local system $\widetilde{\mathscr{E}}_{1}$ on $\widetilde{Y}$, up to isomoprhism, such 
that

$$
\alpha_{0}^{*} \mathscr{E}_{1} \simeq \psi_{0}^{*} \widetilde{\mathscr{E}}_{1}
$$

Since $\alpha_{0}$ is smooth with connected fibre $H \times \mathscr{D}_{P}, \widetilde{\mathscr{E}}_{1}$ turns out to be an $H$-equivariant simple local system on $\tilde{Y}$.

Here $\mathscr{W}=N_{H}\left(L_{H}\right) / L_{H} \simeq S_{a} \ltimes(\mathbf{Z} / 2 \mathbf{Z})^{a^{\prime}}$ (see the proof of Lemma 3.2). Let $\mathscr{W}_{1}$ be the stabilizer of $\Sigma_{\text {reg }} \mathscr{D}_{P}^{0}$ in $\mathscr{W}$. Then $\mathscr{W}_{1} \simeq S_{a}$. We note that $\pi_{0}: \widetilde{Y} \rightarrow Y$ is a finite Galois covering with group $\mathscr{W}_{1}$. In fact, we have an isomorphism $\widetilde{Y} \simeq H \times{ }^{L_{H}} \Sigma_{\text {reg }} \mathscr{D}_{P}^{0}$, and by (3.2.2), the map $\pi_{0}$ is given by the canonical map

$$
\pi_{0}: H \times{ }^{L_{H}} \Sigma_{\text {reg }} \mathscr{D}_{P}^{0} \rightarrow H \times{ }^{N_{H}\left(L_{H}\right)} \zeta^{-1}\left(L_{H}\right),
$$

where $\zeta^{-1}\left(L_{H}\right)=\bigcup_{w \in(\mathbf{Z} / 2 \mathbf{Z})^{a^{\prime}}} w\left(\Sigma_{\text {reg }} \mathscr{D}_{P}^{0}\right) w^{-1}$, see (3.2.3). Thus $\pi_{0}$ is a finite Galois covering with group $\mathscr{W}_{1}$.

We consider $\left(\pi_{0}\right)_{*} \widetilde{\mathscr{E}}_{1}$. Since $\pi_{0}$ is a finite Galois covering, $\left(\pi_{0}\right)_{*} \widetilde{\mathscr{E}}_{1}$ is a semisimple local system on $Y$. Put

$$
\mathscr{W}_{\mathscr{E}_{1}}=\left\{n \in N_{H}\left(L_{H}\right) \mid n\left(\Sigma_{\mathrm{reg}} \mathscr{D}_{P}^{0}\right) n^{-1}=\Sigma_{\mathrm{reg}} \mathscr{D}_{P}^{0}, \operatorname{ad}(n)^{*} \mathscr{E}_{1} \simeq \mathscr{E}_{1}\right\} / L_{H},
$$

where $\operatorname{ad}(n): \Sigma \rightarrow \Sigma, y \mapsto n y n^{-1}$. Then $\mathscr{W}_{\mathscr{E}_{1}}$ is a subgroup of $\mathscr{W}_{1}$. In a similar way as in [L1, Proposition 3.5], one can show that the endomorphism algebra $\mathscr{A}_{\mathscr{E}_{1}}=$ $\operatorname{End}\left(\left(\pi_{0}\right)_{*} \widetilde{\mathscr{E}}_{1}\right)$ of $\left(\pi_{0}\right)_{*} \widetilde{\mathscr{E}}_{1}$ is isomorphic to the (twisted) group algebra $\overline{\mathbf{Q}}_{l}\left[\mathscr{W}_{\mathscr{E}_{1}}\right]$. Thus $\left(\pi_{0}\right)_{*} \widetilde{\mathscr{E}}_{1}$ can be decomposed as

$$
\left(\pi_{0}\right)_{*} \widetilde{\mathscr{E}}_{1} \simeq \bigoplus_{\rho \in \mathscr{A}_{\hat{\mathscr{E}}_{1}}} \rho \otimes\left(\left(\pi_{0}\right)_{*} \widetilde{\mathscr{E}}_{1}\right)_{\rho}
$$

where $\left(\left(\pi_{0}\right)_{*} \widetilde{\mathscr{E}}_{1}\right)_{\rho}=\operatorname{Hom}_{\mathscr{A}_{\mathscr{E}_{1}}}\left(\rho,\left(\pi_{0}\right)_{*} \widetilde{\mathscr{E}}_{1}\right)$ is a simple local system on $Y$ corresponding to $\rho \in \mathscr{A}_{\mathscr{E}_{1}}^{\wedge}$.

3.7. We consider the following commutative diagram

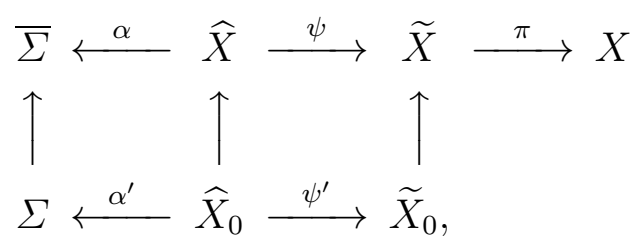

where

$$
\begin{aligned}
\widehat{X} & =\left\{(x, g) \in G^{\iota \theta} \times H \mid g^{-1} x g \in \eta_{P}^{-1}(\bar{\Sigma})\right\} \\
\widehat{X}_{0} & =\left\{(x, g) \in G^{\iota \theta} \times H \mid g^{-1} x g \in \eta_{P}^{-1}(\Sigma)\right\} \\
\widetilde{X}_{0} & =\left\{\left(x, g P_{H}\right) \in G^{\iota \theta} \times H / P_{H} \mid g^{-1} x g \in \eta_{P}^{-1}(\Sigma)\right\}
\end{aligned}
$$

Here $\Sigma, \widehat{X}_{0}, \widetilde{X}_{0}$ are open dense smooth subsets of $\bar{\Sigma}, \widehat{X}, \widetilde{X}$, and the horizontal maps are natural inclusions. $\psi$ is defined by $(x, g) \mapsto\left(x, g P_{H}\right), \alpha$ is defined by $(x, g) \mapsto$ 
$\eta_{P}\left(g^{-1} x g\right)$, and $\psi^{\prime}, \alpha^{\prime}$ are their restrictions on $\widehat{X}_{0}$. $H$ acts on $\tilde{X}$ by $h:\left(x, g P_{H}\right) \mapsto$ $\left(h x h^{-1}, h g P_{H}\right)$, and $H \times P_{H}$ acts on $\widehat{X}$ by $(h, p):(x, g) \mapsto\left(h x h^{-1}, h g p^{-1}\right)$. With the trivial action of $U_{P_{H}}, P_{H}$ acts on $\bar{\Sigma}$, and $\alpha$ is $H \times P_{H}$-equivariant, $\psi$ is $H$-equivariant. Similar properties hold for $\alpha^{\prime}, \psi^{\prime}$ as the restriction of $\alpha, \psi$.

Let $\mathscr{E}_{1}$ be the local system on $\Sigma$ as before. Since $\psi^{\prime}$ is a principal bundle over $\widetilde{X}_{0}$ with respect to the free action of $P_{H}$, by a similar argument as in 3.6, there exists a unique local system (up to isomorphism) $\overline{\mathscr{E}}_{1}$ on $\widetilde{X}_{0}$ such that

$$
\left(\alpha^{\prime}\right)^{*} \mathscr{E}_{1} \simeq\left(\psi^{\prime}\right)^{*} \overline{\mathscr{E}}_{1}
$$

Note that $\tilde{Y}$ is an open dense subset of $\widetilde{X}_{0}$, and the restriction of $\overline{\mathscr{E}}_{1}$ on $\tilde{Y}$ coincides with $\widetilde{\mathscr{E}}_{1}$ defined in 3.6. Put $K_{\overline{\mathscr{E}}_{1}}=\operatorname{IC}\left(\widetilde{X}, \overline{\mathscr{E}}_{1}\right)[\operatorname{dim} \tilde{X}]$. Then $K_{\overline{\mathscr{E}}_{1}}$ is an $H$-equivariant simple perverse sheaf on $\widetilde{X}_{0}$. Since $\alpha$ is smooth with connected fibre $H \times U_{P}^{\iota \theta}$, and $\psi$ is a principal bundle with respect to the free action of $P_{H}$, the discussion in 3.6 works in the level of perverse sheaves, and we have

$$
\alpha^{*} \mathrm{IC}\left(\bar{\Sigma}, \mathscr{E}_{1}\right)[\operatorname{dim} \Sigma] \simeq \psi^{*} \operatorname{IC}\left(\widetilde{X}, \overline{\mathscr{E}}_{1}\right)[\operatorname{dim} \tilde{X}]
$$

We consider the complex $\pi_{*} K_{\overline{\mathscr{E}}_{1}}$ on $X=\bar{Y}$. The following result is an analogue of [L1, Proposition 4.5]. Note that here we don't need to assume that $\left(\Sigma, \mathscr{E}_{1}\right)$ is cuspidal.

Proposition 3.8. We have $\pi_{*} K_{\overline{\mathscr{E}}_{1}} \simeq \operatorname{IC}\left(X,\left(\pi_{0}\right)_{*} \widetilde{\mathscr{E}}_{1}\right)[\operatorname{dim} X]$.

Proof. By Lemma 3.5 and Lemma 3.2, we have $\operatorname{dim} X=2 \nu_{H}-2 \nu_{L_{H}}+\operatorname{dim} \Sigma+\Delta_{P}$. Then the proof of the proposition is done by an entirely similar way as the proof of Proposition 4.5 in [L1], by making use of Proposition 2.2. We omit the details.

3.9. By Proposition 3.8, $\pi_{*} K_{\overline{\mathscr{E}}_{1}}$ is a semisimple perverse sheaf. By (3.6.1), it is decomposed as

$$
\pi_{*} K_{\overline{\mathscr{E}}_{1}} \simeq \bigoplus_{\rho \in \mathscr{A}_{\hat{\mathscr{E}}_{1}}} \rho \otimes \operatorname{IC}\left(X,\left(\left(\pi_{0}\right)_{*} \widetilde{\mathscr{E}}_{1}\right)_{\rho}\right)[\operatorname{dim} X]
$$

A simple perverse sheaf isomorphic to a direct summand of $\pi_{*} K_{\overline{\mathscr{E}}_{1}}$ obtained from the various cuspidal pair $\left(\Sigma, \mathscr{E}_{1}\right)$ is called an admissible complex.

Remark 3.10. In the case where $P=B$ and $L=T$, we have $\Sigma=T^{\iota \theta}$. Then

$$
\begin{aligned}
& \tilde{X}=\left\{\left(x, g B_{H}\right) \in G^{\iota \theta} \times H / B_{H} \mid g^{-1} x g \in B^{\iota \theta}\right\}, \\
& X=\bigcup_{g \in H} g B^{\iota \theta} g^{-1},
\end{aligned}
$$

and $\pi: \widetilde{X} \rightarrow X$ is the first projection. A similar map can be defined even in the case where $H=S p_{N}$. However in that case, we have $\operatorname{dim} \tilde{X}>\operatorname{dim} X$, and 
Lemma 3.5 does not hold. Nevertheless, in the case where $\mathscr{E}_{1}$ is a constant sheaf $\overline{\mathbf{Q}}_{l}$ on $T^{\iota \theta}$, it was proved in $[\mathrm{H}]$ (see also $[\mathrm{SS}]$ ) that $\pi_{*} \overline{\mathbf{Q}}_{l}$ is a semisimple complex equipped with $S_{n}$-action, and some modified formula of (3.9.1) holds. If we consider the exotic symmetric space $G^{\iota \theta} \times V$ associated to the symplectic group $S p(V)$, the map $\pi: \widetilde{X} \rightarrow X$ is also defined. In that case, an exactly analogous formula of (3.9.1) holds $([\mathrm{SS}],[\mathrm{K}])$.

\section{ShEAVES ON THE VARIETY OF SEMISIMPLE ORBITS}

4.1. We keep the setting in Section 3. In particular, consider $Y=Y_{(L, \Sigma)}$ and $X=\bar{Y}$ with $\Sigma=Z_{L}^{\iota \theta} \mathscr{O}_{L}$. In this section, we assume that the local system $\mathscr{E}_{1}$ on

$\Sigma$ is of the form $\mathscr{E}_{1}=\overline{\mathbf{Q}}_{l} \otimes \mathscr{E}_{1}^{\dagger}$, where $\overline{\mathbf{Q}}_{l}$ is the constant sheaf on $Z_{L}^{\iota \theta}$ and $\mathscr{E}_{1}^{\dagger}$ is an $L_{H}$-equivariant simple local system on $\mathscr{O}_{L}$.

Let $\sigma^{\prime}: G \rightarrow T / S_{N}$ be the Steinberg map with respect to $G=G L_{N}$. We have a natural embedding $A=T^{\iota \theta} / S_{n} \hookrightarrow T / S_{N}$, where $A$ has a structure of an affine variety. Let $G_{+}^{\iota \theta}$ be the subset of $G^{\iota \theta}$ consisting of $g \in G^{\iota \theta}$ such that its semisimple part $g_{s}$ is $H$-conjugate to an element in $T^{\iota \theta}$. (Note that $T^{\iota \theta}$ is not a maximal $\theta$ anisotropic torus. See the example in 1.4, which corresponds to our setting with $N=2$.) Then the restriction of $\sigma^{\prime}$ on $G_{+}^{\iota \theta}$ induces a map $\sigma: G_{+}^{\iota \theta} \rightarrow A$. Since $\bar{Y} \subset G_{+}^{\iota \theta}$, one can consider $\sigma(\bar{Y})$. We have

$$
\sigma(\bar{Y})=\sigma\left(\eta_{P}^{-1}(\bar{\Sigma})\right)=\sigma(\bar{\Sigma})=\sigma\left(Z_{L}^{\iota \theta}\right) \subset A,
$$

which we denote by $A_{Y}$.

4.2. Let $\left(B^{\prime}, T^{\prime}\right)$ be another $\theta$-stable pair. By 1.9 , the $H$-conjugate class of $\left(B^{\prime}, T^{\prime}\right)$ is described as follows. If $N$ is odd, we may choose $\left(B^{\prime}, T^{\prime}\right)=(B, T)$. If $N$ is even, then $\left(B^{\prime}, T^{\prime}\right)=(B, T)$ or $B^{\prime}=t_{n} B t_{n}^{-1}, T^{\prime}=T$, where $t_{n} \in N_{G^{\theta}}(T)$ is given as in 1.9. We put $B_{1}=t_{n} B t_{n}^{-1}$.

Assume that $N$ is even, and let $P$ be a $\theta$-stable parabolic subgroup of $G$ containing $B$, and $L$ the Levi subgroup of $P$ containing $T$. Then $P^{\prime}=t_{n} P t_{n}^{-1}$ is the $\theta$-stable parabolic subgroup containing $B_{1}$, and $L^{\prime}=t_{n} L t_{n}^{-1}$ is the Levi subgroup of $P^{\prime}$ containing $T$. Note that $L^{\prime}=L$ if $L \neq T$.

4.3. We now consider another $\theta$-stable parabolic subgroup $P^{\prime}$ containing $B^{\prime}$ and its $\theta$-stable Levi subgroup $L^{\prime}$ containing $T$, where $B^{\prime}=B$ or $B^{\prime}=B_{1}$. We consider a set $\Sigma^{\prime} \subset L^{\prime \prime \theta}$ similarly to $\Sigma \subset L^{\iota \theta}$. We shall denote by $Y^{\prime}, X^{\prime}$, etc. various objects associated to $\Sigma^{\prime}, L^{\prime}, P^{\prime}$ by attaching prime to corresponding objects $Y, X$, etc. associated to $\Sigma, L, P$. Put

$$
\left.Z=\left\{\left(x, g P_{H}\right),\left(x^{\prime}, g^{\prime} P_{H}^{\prime}\right)\right) \in \tilde{X} \times \tilde{X}^{\prime} \mid x=x^{\prime}\right\}
$$

Thus $Z$ is isomorphic to the fibre product $\tilde{X} \times_{G^{\iota \theta}} \tilde{X}^{\prime}$ of $\widetilde{X}$ and $\widetilde{X}^{\prime}$ over $G^{\iota \theta}$. Here we assume that $X \cap X^{\prime} \neq \emptyset$, otherwise $Z=\emptyset$. We define a map $\tilde{\sigma}: Z \rightarrow A_{Y} \cap A_{Y^{\prime}}$ by the composite $\sigma \circ p_{1}$ of the first projection $p_{1}: Z \rightarrow X \cap X^{\prime}$ and $\sigma$. For $a \in A_{Y}$, put $Z^{a}=\tilde{\sigma}(a)^{-1} \subset Z$. 
Since $\bar{\Sigma}=Z_{L}^{\iota \theta} \overline{\mathscr{O}}_{L}, \bar{\Sigma}$ has a stratification $\bar{\Sigma}=\coprod_{\beta} \Sigma_{\beta}$ with smooth strata $\Sigma_{\beta}=$ $Z_{L}^{\iota \theta} \mathscr{O}_{\beta}$, where we put $\overline{\mathscr{O}}_{L}=\coprod_{\beta} \mathscr{O}_{\beta}\left(\mathscr{O}_{\beta}: L_{H^{-}}\right.$orbit in $\left.L_{\text {uni }}^{\iota \theta}\right)$. We put $\Sigma_{\beta_{0}}=\Sigma=$ $Z_{L}^{\iota \theta} \mathscr{O}_{L}$ : the open dense stratum. By defining $\widetilde{X}_{\beta}$ in a similar way as $\tilde{X}_{0}$ in 3.7 , we obtain a stratification $\widetilde{X}=\coprod_{\beta} \widetilde{X}_{\beta}$, with $\widetilde{X}_{0}=\widetilde{X}_{\beta_{0}}$, where $\tilde{X}_{\beta}$ are locally closed subvarieties of $\tilde{X}$. Given strata $\beta$ of $\bar{\Sigma}$ and $\beta^{\prime}$ of $\bar{\Sigma}^{\prime}$, we put $Z_{\beta, \beta^{\prime}}^{a}=Z^{a} \cap\left(\widetilde{X}_{\beta} \times_{G^{\iota \theta}} \widetilde{X}_{\beta^{\prime}}^{\prime}\right)$. Then the sets $Z_{\beta, \beta^{\prime}}^{a}$ form a partition of $Z^{a}$ into locally closed pieces, where $Z_{\beta_{0}, \beta_{0}^{\prime}}^{a}$ is open dense in $Z^{a}$. Put

$$
d_{0}=2 \nu_{H}-\nu_{L_{H}}-\nu_{L_{H}^{\prime}}+\left(\operatorname{dim} \mathscr{O}_{L}+\operatorname{dim} \mathscr{O}_{L^{\prime}}\right) / 2+\left(\Delta_{P}+\Delta_{P^{\prime}}\right) / 2
$$

Take local systems $\mathscr{E}_{1}$ on $\Sigma$ and $\mathscr{E}_{1}^{\prime}$ on $\Sigma^{\prime}$, and consider the external tensor product $K_{\mathscr{E}_{1}} \otimes K_{\overline{\mathscr{E}}_{1}^{\prime}}^{\prime}$ on $Z$. We define

$$
\widetilde{\mathscr{T}}=\mathscr{H}^{-r-r^{\prime}}\left(\widetilde{\sigma}_{!}\left(K_{\overline{\mathscr{E}}_{1}} \otimes K_{\overline{\mathscr{E}}_{1}^{\prime}}^{\prime}\right)\right)
$$

where we put $r=\operatorname{dim} Z_{L}^{\iota \theta}, r^{\prime}=\operatorname{dim} Z_{L^{\prime}}^{\iota \theta}$. $\widetilde{\mathscr{T}}$ is a constructible sheaf on $A_{Y} \cap A_{Y^{\prime}}$.

Put $Z_{0}=\widetilde{X}_{0} \times_{G^{\iota} \theta} \tilde{X}_{0}^{\prime}$. Then $Z_{0}$ is an open dense subset of $Z$. Let $\widetilde{\sigma}_{0}: Z_{0} \rightarrow$ $A_{Y} \cap A_{Y^{\prime}}$ be the restriction of $\widetilde{\sigma}$ on $Z_{0}$. Noticing that

$$
\left.K_{\overline{\mathscr{E}}_{1}} \otimes K_{\overline{\mathscr{E}}_{1}^{\prime}}^{\prime}\left[-\operatorname{dim} X-\operatorname{dim} X^{\prime}\right]\right|_{Z_{0}}=\overline{\mathscr{E}}_{1} \otimes \overline{\mathscr{E}}_{1}^{\prime}
$$

we define

$$
\mathscr{T}=\mathscr{H}^{-r-r^{\prime}}\left(\left(\widetilde{\sigma}_{0}\right) !\left(\left.K_{\overline{\mathscr{E}}_{1}} \otimes K_{\overline{\mathscr{E}}_{1}^{\prime}}^{\prime}\right|_{Z_{0}}\right)=\mathscr{H}^{2 d_{0}}\left(\left(\widetilde{\sigma}_{0}\right)_{!}\left(\overline{\mathscr{E}}_{1} \otimes \overline{\mathscr{E}}_{1}^{\prime}\right)\right)\right.
$$

$\mathscr{T}$ is also a constructible sheaf on $A_{Y} \cap A_{Y^{\prime}}$. Since $Z_{0}$ is open in $Z$, we have a natural map $\mathscr{T} \rightarrow \widetilde{\mathscr{T}}$. In order to obtain a relationship between $\widetilde{\mathscr{T}}$ and $\mathscr{T}$, we prepare a lemma, which is an analogue of [L1, Lemma 5.3]. The proof is done by a similar argument as in the proof of [loc. cit.], by using Proposition 2.2, and we omit the proof.

Lemma 4.4. (i) For any $\beta, \beta^{\prime}$, we have

$$
\operatorname{dim} Z_{\beta, \beta^{\prime}}^{a} \leq d_{0}-\left(\operatorname{dim} \mathscr{O}_{L}+\operatorname{dim} \mathscr{O}_{L^{\prime}}\right) / 2+\left(\operatorname{dim} \mathscr{O}_{\beta}+\operatorname{dim} \mathscr{O}_{\beta^{\prime}}\right) / 2
$$

In particular, we have $\operatorname{dim} Z^{a} \leq d_{0}$.

(ii) The natural map

$$
H_{c}^{k+\operatorname{dim} X+\operatorname{dim} X^{\prime}}\left(Z_{\beta_{0}, \beta_{0}}^{a}, \overline{\mathscr{E}}_{1} \otimes \overline{\mathscr{E}}_{1}^{\prime}\right) \simeq \mathbf{H}_{c}^{k}\left(Z_{\beta_{0}, \beta_{0}}^{a}, K_{\overline{\mathscr{E}}_{1}} \otimes K_{\overline{\mathscr{E}}_{1}^{\prime}}\right) \rightarrow \mathbf{H}_{c}^{k}\left(Z^{a}, K_{\overline{\mathscr{E}}_{1}} \otimes K_{\overline{\mathscr{E}}_{1}^{\prime}}\right)
$$

is an isomorphism for $k>-r-r^{\prime}$, and is surjective if $k=-r-r^{\prime}$. It is an isomorphism for $k=-r-r^{\prime}$ (i.e, for $k+\operatorname{dim} X+\operatorname{dim} X^{\prime}=2 d_{0}$ ) if a is such that $Z_{\beta, \beta^{\prime}}^{a}$ is empty whenever exactly one of $\beta, \beta^{\prime}$ is equal to $\beta_{0}$ or $\beta_{0}^{\prime}$. 
4.5. Let $\widetilde{\mathscr{T}_{a}}$ (resp. $\mathscr{T}_{a}$ ) be the stalk of $\widetilde{\mathscr{T}}($ resp. $\mathscr{T})$ for $a \in A_{Y} \cap A_{Y^{\prime}}$. Then we have

$$
\begin{aligned}
& \widetilde{\mathscr{T}_{a}} \simeq \mathbf{H}_{c}^{-r-r^{\prime}}\left(Z^{a}, K_{\overline{\mathscr{E}}_{1}} \otimes K_{\overline{\mathscr{E}}_{1}^{\prime}}^{\prime}\right), \\
& \mathscr{T}_{a} \simeq \mathbf{H}_{c}^{-r-r^{\prime}}\left(Z_{\beta_{0}, \beta_{0}^{\prime}}^{a}, K_{\overline{\mathscr{E}}_{1}} \otimes K_{\overline{\mathscr{E}}_{1}^{\prime}}^{\prime}\right)
\end{aligned}
$$

and the natural map $\mathscr{T}_{a} \rightarrow \widetilde{\mathscr{T}}_{a}$ corresponds to the map in Lemma 4.4 (ii) for $k=$ $-r-r^{\prime}$. Thus, by Lemma 4.4 (ii), we see that

(4.5.1) The natural map of sheaves $\mathscr{T} \rightarrow \widetilde{\mathscr{T}}$ is surjective.

Let $\mathbb{O}$ be an $H$-orbit in $H / P_{H} \times H / P_{H}^{\prime}$, and put $Z_{0}^{\mathbb{O}}=q^{-1}(\mathbb{O})$, where $q: Z_{0} \rightarrow$ $H / P_{H} \times H / P_{H}^{\prime}$ is the projection $\left(x, g P_{H}, g^{\prime} P_{H}^{\prime}\right) \mapsto\left(g P_{H}, g^{\prime} P_{H}^{\prime}\right)$. Put

$$
\mathscr{T}_{\mathbb{O}}=\mathscr{H}^{-r-r^{\prime}}(\widetilde{\sigma}) !\left(\left.K_{\overline{\mathscr{E}}_{1}} \otimes K_{\overline{\mathscr{E}}_{1}^{\prime}}^{\prime}\right|_{Z_{0}^{\mathbb{Q}}}\right)=\mathscr{H}^{2 d_{0}}\left(\widetilde{\sigma}_{0}\right) !\left(\left.\overline{\mathscr{E}}_{1} \otimes \overline{\mathscr{E}}_{1}^{\prime}\right|_{Z_{0}^{\mathbb{Q}}}\right)
$$

$\mathscr{T}_{\mathbb{O}}$ is a constructible sheaf on $A_{Y} \cap A_{Y^{\prime}}$. Recall that $\mathscr{W}=N\left(L_{H}\right) / L_{H}$ and $\mathscr{W}_{1} \simeq S_{a}$ is the subgroup of $\mathscr{W}$. Let $N_{H}\left(L_{H}\right)_{1}$ be the inverse image of $\mathscr{W}_{1}$ under the $\operatorname{map} N_{H}\left(L_{H}\right) \rightarrow \mathscr{W}$

We show a lemma.

Lemma 4.6. Assume that $\left(\Sigma, \mathscr{E}_{1}\right),\left(\Sigma^{\prime}, \mathscr{E}_{1}^{\prime}\right)$ are cuspidal pairs. Let $\mathbb{O}=\mathbb{O}_{\omega}$ be an $H$-orbit corresponding to $\omega \in W_{L_{H}} \backslash W_{H} / W_{L_{H}^{\prime}}$, and $w \in W_{H}$ be a representative of $\omega$. Let $\dot{w} \in N_{H}\left(T_{H}\right)$ be a representative of $w$.

(i) If $(L, \Sigma)$ and $\left(L^{\prime}, \Sigma^{\prime}\right)$ are not conjugate under $H$, then $\mathscr{T}_{\mathbb{O}}=0$.

(ii) Assume that $P=P^{\prime}, L=L^{\prime}$ and $\Sigma=\Sigma^{\prime}$. If $\dot{w} \in N_{H}\left(T_{H}\right)$ is not contained in $N_{H}\left(L_{H}\right)_{1}$. Then $\mathscr{T}_{\mathbb{O}}=0$.

(iii) Assume that $N$ is even, and $P=B, P^{\prime}=B_{1}, L=L^{\prime}=T$. Then $\mathscr{T}_{\mathbb{O}}=0$.

Proof. In order to see $\mathscr{T}_{\mathbb{O}}=0$, we have only to show that, for any $a \in A_{Y} \cap A_{Y^{\prime}}$,

$$
H_{c}^{2 d_{0}}\left(Z^{a} \cap Z_{0}^{\mathbb{O}}, \overline{\mathscr{E}}_{1} \otimes \overline{\mathscr{E}}_{1}^{\prime}\right)=0
$$

Since $\operatorname{dim}\left(Z^{a} \cap Z_{0}^{\mathbb{Q}}\right) \leq d_{0}$ (Lemma $4.4(\mathrm{i})$ ), by using the fibration $Z^{a} \cap Z_{0}^{\mathbb{O}} \rightarrow \mathbb{O}$, it is enough to see, for any $a \in A_{Y} \cap A_{Y^{\prime}}$ and for any $g \in H, H_{c}^{2 d_{0}-2 \operatorname{dim} \mathbb{O}}\left(V^{a}, j^{*}\left(\mathscr{E}_{1} \otimes\right.\right.$ $\left.\left.\mathscr{E}_{1}^{\prime}\right)\right)=0$, where $\mathbb{O}$ is the $H$-orbit containing $\left(P_{H}, \dot{w} P_{H}^{\prime}\right)$ (here $\dot{w} \in N_{H}\left(T_{H}\right)$ is a representative of $w \in W_{H}$ ), and

$$
V^{a}=\left\{x \in \sigma^{-1}(a) \mid g^{-1} x g \in \eta_{P}^{-1}(\Sigma), \dot{w}^{-1} g^{-1} x g \dot{w} \in \eta_{P^{\prime}}^{-1}\left(\Sigma^{\prime}\right)\right\},
$$

and $j: V^{a} \rightarrow \Sigma \times \Sigma^{\prime}$ is defined by $j(x)=\left(\eta_{P}\left(g^{-1} x g\right), \eta_{P^{\prime}}\left(\dot{w}^{-1} g^{-1} x g \dot{w}\right)\right)$. We have $g^{-1} x g \in P^{\iota \theta} \cap{ }^{w} P^{\prime \iota \theta}$. As in the proof of Proposition 2.2, one can write $g^{-1} x g$ as $g^{-1} x g=\left(z y^{\prime}\right) \cdot u=(z y) \cdot u^{\prime}$, where $z \in L \cap{ }^{w} L^{\prime}, y^{\prime} \in L \cap{ }^{w} U_{P^{\prime}}, y \in{ }^{w} L^{\prime} \cap U_{P}, u \in U_{P}^{\iota \theta}$, 
$u^{\prime} \in{ }^{w} U_{P^{\prime}}^{\iota \theta}$. Thus $V^{a}$ can be described as follows; put

$$
\begin{aligned}
\tilde{V}^{a}= & \left\{\left(u, u^{\prime}, y, y^{\prime}, z\right) \in U_{P}^{\iota \theta} \times{ }^{w} U_{P^{\prime}}^{\iota \theta} \times\left({ }^{w} L^{\prime} \cap U_{P}\right) \times\left(L \cap{ }^{w} U_{P^{\prime}}\right) \times\left(L \cap{ }^{w} L^{\prime}\right)\right. \\
& \left.\mid y^{\prime} \cdot u=y \cdot u^{\prime},\left(z y^{\prime}\right) \theta\left(z y^{\prime}\right)^{-1} \in \Sigma \cap \sigma^{-1}(a),(z y) \theta(z y)^{-1} \in \dot{w} \Sigma^{\prime} \dot{w}^{-1} \cap \sigma^{-1}(a)\right\} .
\end{aligned}
$$

Then $V^{a}$ is isomorphic to the quotient of $\widetilde{V}^{a}$ by $E^{\theta}$, where $E=\left({ }^{w} L^{\prime} \cap U_{P}\right) \times(L \cap$ $\left.{ }^{w} U_{P^{\prime}}\right) \times\left(L \cap{ }^{w} L^{\prime}\right)$ and the action of $E^{\theta}$ is given by the same formula as in the proof of Proposition 2.2. We now consider the map $\widetilde{V}^{a} / E^{\theta} \rightarrow E / E^{\theta}$ induced by the projection $\left(u, u^{\prime}, y, y^{\prime}, z\right) \mapsto\left(y, y^{\prime}, z\right)$. Its image is given by

$$
\begin{array}{r}
\bar{V}^{a}=\left\{\left(y, y^{\prime}, z\right) \in E \mid\left(z y^{\prime}\right) \theta\left(z y^{\prime}\right)^{-1} \in \Sigma \cap \sigma^{-1}(a),\right. \\
\left.(z y) \theta(z y)^{-1} \in \dot{w} \Sigma^{\prime} \dot{w}^{-1} \cap \sigma^{-1}(a)\right\} / E^{\theta} .
\end{array}
$$

By a similar computation as in (2.2.4), we see that all the fibres of this map are isomorphic to $\left(U_{P} \cap{ }^{w} U_{P^{\prime}}\right)^{\iota \theta}$. Moreover, by a similar discussion as in the proof of (2.2.8), one can show that

$$
\operatorname{dim}\left(U_{P} \cap{ }^{w} U_{P^{\prime}}\right)^{\iota \theta}=2 \nu_{H}-\nu_{L_{H}}-\nu_{L_{H}^{\prime}}-\operatorname{dim} \mathbb{O}_{\omega}+b_{w}^{\prime},
$$

where

$$
b_{w}^{\prime}=\sharp\left\{i \mid 1 \leq i \leq n-n_{0}, 1 \leq w^{-1}(i) \leq n-n_{0}^{\prime}\right\} .
$$

(Here we assume that $L^{\theta} \simeq\left(G L_{1}\right)^{\left(N-N_{0}\right) / 2} \times G L_{N_{0}}^{\theta}$ and $L^{\prime \theta} \simeq\left(G L_{1}\right)^{\left(N-N_{0}^{\prime}\right) / 2} \times G L_{N_{0}^{\prime}}^{\theta}$, and put $n_{0}=\left[N_{0} / 2\right], n_{0}^{\prime}=\left[N_{0}^{\prime} / 2\right]$. If $B^{\prime}=B$, a similar argument as in the proof of Proposition 2.2 can be applied. In the case where $N$ is even, we need to consider $B^{\prime}=B_{1}$ also. But in this case, if we replace $W_{H}=N_{H}\left(T_{H}\right) / T_{H}$ by a group $N_{G^{\theta}}\left(T_{H}\right) / T_{H}$, which is isomorphic to $S_{n} \ltimes(\mathbf{Z} / 2 \mathbf{Z})^{n}$, a similar argument as in the odd $N$ case works.)

Thus we are reduced to showing that

$$
H_{c}^{s}\left(\bar{V}^{a}, \bar{j}^{*}\left(\mathscr{E}_{1} \otimes \mathscr{E}_{1}^{\prime}\right)\right)=0
$$

where $s=\operatorname{dim} \mathscr{O}_{L}+\operatorname{dim} \mathscr{O}_{L^{\prime}}+\Delta_{P}+\Delta_{P^{\prime}}-2 b_{w}^{\prime}$, and $\bar{j}: \bar{V}^{a} \rightarrow \Sigma \times \Sigma^{\prime}$ is defined by $\left(y, y^{\prime}, z\right) \mapsto\left((z y) \theta(z y)^{-1}, \dot{w}^{-1}\left(z y^{\prime}\right) \theta\left(z y^{\prime}\right)^{-1} \dot{w}\right)$

We use the notation $Q=L \cap{ }^{w} P^{\prime}$ the $\theta$-stable parabolic subgroup of $L$ with Levi decomposition $Q=M U_{Q}$, where $M=L \cap{ }^{w} L^{\prime}, U_{Q}=L \cap{ }^{w} U_{P^{\prime}}$. Similarly, we define $Q^{\prime}={ }^{w} L^{\prime} \cap P$ the $\theta$-stable parabolic subgroup of ${ }^{w} L^{\prime}$ with Levi decomposition $Q^{\prime}=M U_{Q^{\prime}}$, where $U_{Q^{\prime}}={ }^{w} L^{\prime} \cap U_{P}$. Let $\pi_{3}: \bar{V}^{a} \rightarrow M^{\iota \theta}$ be the map defined by $\left(y, y^{\prime}, z\right) \mapsto z \theta(z)^{-1}$. Since the semisimple part of $z \theta(z)^{-1}$ is contained in a fixed $H$-orbit, $\pi_{3}\left(\bar{V}^{a}\right)$ consists of finitely many $\left(M^{\theta}\right)^{0}$-orbits $\widehat{\mathscr{O}}_{1}, \ldots, \widehat{\mathscr{O}}_{m}$ in $M^{\iota \theta}$. Since $\operatorname{dim} \bar{V}^{a} \leq s / 2$, it is enough to show, for any $i$, that $H_{c}^{s-2 \operatorname{dim} \widehat{\mathscr{O}}_{i}}\left(\pi_{3}^{-1}(\xi), \bar{j}^{*}\left(\mathscr{E}_{1} \bigotimes \mathscr{E}_{1}^{\prime}\right)\right)=0$ 
for $\xi \in \widehat{\mathscr{O}}_{i}$. Here $\pi_{3}^{-1}(\xi)$ is isomorphic to $D \times D^{\prime}$, where

$$
\begin{aligned}
D & =\left\{q \in Q \cap \Sigma \mid \eta_{Q}(q)=\xi\right\}, \\
D^{\prime} & =\left\{q^{\prime} \in Q^{\prime} \cap \dot{w} \Sigma^{\prime} \dot{w}^{-1} \mid \eta_{Q^{\prime}}\left(q^{\prime}\right)=\xi\right\} .
\end{aligned}
$$

Moreover, the restriction of $\bar{j}^{*}\left(\mathscr{E}_{1} \bigotimes \mathscr{E}_{1}^{\prime}\right)$ on $\pi_{3}^{-1}(\xi)$ corresponds to the tensor product $\left.\left.\mathscr{E}_{1}\right|_{D} \otimes n^{*} \mathscr{E}_{1}^{\prime}\right|_{D^{\prime}}$ (here $n: \dot{w} \Sigma^{\prime} \dot{w}^{-1} \underset{\rightarrow}{\rightarrow} \Sigma^{\prime}$ ). Here by Proposition 2.2 (i), $2 \operatorname{dim} D \leq d=$ $\operatorname{dim} \mathscr{O}_{L}-\operatorname{dim} \widehat{\mathscr{O}}_{i}+\Delta_{Q}, 2 \operatorname{dim} D^{\prime} \leq d^{\prime}=\operatorname{dim} \mathscr{O}_{L^{\prime}}-\operatorname{dim} \widehat{\mathscr{O}}_{i}+\Delta_{Q^{\prime}}$. In a similar way as in (2.2.12), we have

$$
\Delta_{Q}+b_{w}^{\prime} \leq \Delta_{P^{\prime}}, \quad \Delta_{Q^{\prime}}+b_{w}^{\prime} \leq \Delta_{P}
$$

since one can write as

$$
\begin{aligned}
& \Delta_{Q}=\sharp\left\{i \mid n-n_{0}+1 \leq i \leq n, 1 \leq w^{-1}(i) \leq n-n_{0}^{\prime}\right\}, \\
& \Delta_{Q^{\prime}}=\sharp\left\{i \mid n-n_{0}^{\prime}+1 \leq i \leq n, 1 \leq w(i) \leq n-n_{0}\right\} .
\end{aligned}
$$

It follows that

$$
d+d^{\prime}=s-2 \operatorname{dim} \widehat{\mathscr{O}}_{i}+\Delta_{Q}+\Delta_{Q^{\prime}}-\Delta_{P}-\Delta_{P^{\prime}}+2 b_{w}^{\prime} \leq s-2 \operatorname{dim} \widehat{\mathscr{O}}_{i}
$$

Hence by the Künneth formula we are reduced to showing

$$
H_{c}^{d}\left(D, \mathscr{E}_{1}\right) \otimes H_{c}^{d^{\prime}}\left(D^{\prime}, n^{*} \mathscr{E}_{1}^{\prime}\right)=0
$$

Unless $L={ }^{w} L^{\prime}, Q=L \cap{ }^{w} P^{\prime}$ is a proper parabolic subgroup of $L$, or $Q^{\prime}={ }^{w} L^{\prime} \cap P$ is a proper parabolic subgroup of ${ }^{w} L^{\prime}$. In that case, since the local systems $\mathscr{E}_{1}, \mathscr{E}_{1}^{\prime}$ are cuspidal, we have $H_{c}^{d}\left(D, \mathscr{E}_{1}\right)=0$ or $H_{c}^{d^{\prime}}\left(D^{\prime}, n^{*} \mathscr{E}_{1}^{\prime}\right)=0$ by Lemma 2.5. Thus (4.6.2), and so (4.6.1) holds. Now assume that $B^{\prime}=B$. Then by the choice of $P$ and $P^{\prime}$, we have $L=L^{\prime}$ and $\dot{w} \in N_{H}\left(L_{H}\right)$. If $\Sigma \cap \Sigma^{\prime}=\emptyset$, then $V^{a}=\emptyset$, so (4.6.1) holds. This proves (i). Assume that $\dot{w} \in N_{H}\left(L_{H}\right)$, but $\dot{w} \notin N_{H}\left(L_{H}\right)_{1}$. Then $Q=L$ and $\Delta_{Q}=0$. Moreover $\Delta_{P}>b_{w}^{\prime}$. Hence $d+d^{\prime}<s-2 \operatorname{dim} \widehat{\mathscr{O}}_{i}$. This implies that $H_{c}^{s-2 \operatorname{dim} \widehat{\mathscr{O}}_{i}}\left(\pi_{3}^{-1}(\xi), \bar{j}^{*}\left(\mathscr{E}_{1} \otimes \mathscr{E}_{1}^{\prime}\right)\right)=0$, and so (4.6.1) holds. This proves (ii). Next assume that $N$ is even and $B^{\prime}=B_{1}$. If $L \neq T$, we have $L^{\prime}=L$ (see 4.2), and $\dot{w} \in N_{H}\left(L_{H}\right)$. The same argument as before works, and (i), (ii) are proved. So assume that $L=L^{\prime}=T$, and $B^{\prime}=B_{1}$. This case is essentially the same as the case where $B^{\prime}=B$, and $\dot{w} \in N_{G^{\theta}}\left(T_{H}\right)$. We see that if $\dot{w} \in N_{G^{\theta}}\left(T_{H}\right)-N_{H}\left(T_{H}\right)_{1}$, then $b_{w}^{\prime}=\sharp\left\{1 \leq i \leq n \mid w^{-1}(i)>0\right\}<\Delta_{B}=n$. Hence $d+d^{\prime}<s$, and (4.6.2) holds. This proves (iii). The lemma is proved.

4.7. Following [L1, 5.4], we recall the notion of perfect sheaves. A constructible sheaf $\mathscr{E}$ on an irreducible variety $V$ is said to be perfect, if

(i) $\mathscr{E}=\mathrm{IC}\left(V,\left.\mathscr{E}\right|_{V_{0}}\right)$, where $V_{0}$ is an open dense smooth subset of $V$ and $\left.\mathscr{E}\right|_{V_{0}}$ is locally constant, 
(ii) the support of any non-zero constructible subsheaf of $\mathscr{E}$ is dense in $V$.

In particular, the complex $\operatorname{IC}\left(V,\left.\mathscr{E}\right|_{V_{0}}\right)$ is reduced to a single sheaf. The following properties hold.

(4.7.1) If $\pi: V^{\prime} \rightarrow V$ is a finite morphism, with $V^{\prime}$ smooth, and if $\mathscr{E}^{\prime}$ is a locally constant sheaf on $V^{\prime}$, then $\mathscr{E}=\pi_{*} \mathscr{E}^{\prime}$ is a perfect sheaf on $V$.

(4.7.2) If $0 \rightarrow \mathscr{E}_{1} \rightarrow \mathscr{E}_{2} \rightarrow \mathscr{E}_{3} \rightarrow 0$ is an exact sequence of constructible sheaves on $V$, with $\mathscr{E}_{1}, \mathscr{E}_{3}$ perfect, then $\mathscr{E}_{2}$ is perfect.

4.8. We assume that $(L, \Sigma)=\left(L^{\prime}, \Sigma^{\prime}\right)$. We also assume $\mathbb{O}=\mathbb{O}_{\omega}$, where a representative of $\omega$ satisfies the condition $n=\dot{w} \in N_{H}\left(L_{H}\right)$ with $w \in \mathscr{W}_{1}$. In this case, we have $n \Sigma n^{-1}=\Sigma$. The computation in the proof of the lemma shows that

$$
\mathscr{T}_{\mathbb{O}} \simeq R^{s} \bar{\sigma}_{!}\left(\mathscr{E}_{1} \otimes n^{*} \mathscr{E}_{1}^{\prime}\right)
$$

where $\bar{\sigma}: \Sigma \rightarrow A_{Y}$ is the restriction of $\sigma, s=2 \operatorname{dim} \mathscr{O}_{L}$ (note that $\Delta_{P}=b_{w}^{\prime}=b_{w}$ ). Here $\Sigma=Z_{L}^{\iota \theta} \times \mathscr{O}_{L}$. We denote by $\pi_{1}: \Sigma \rightarrow Z_{L}^{\iota \theta}$ the projection, and $\bar{\sigma}_{1}: Z_{L}^{\iota \theta} \rightarrow A_{Y}$ the restriction of $\sigma$. Since $\bar{\sigma}_{1}$ is a finite morphism, if we put $\mathscr{G}=R^{s}\left(\pi_{1}\right) !\left(\mathscr{E}_{1} \otimes n^{*} \mathscr{E}_{1}^{\prime}\right)$, we have

$$
\mathscr{F}_{\mathbb{O}}=\left(\bar{\sigma}_{1}\right)_{*} \mathscr{G}
$$

We note that

(4.8.2) $\mathscr{G}$ is a locally constant sheaf on $Z_{L}^{\iota \theta}$.

In fact, $Z_{L}^{\iota \theta}$ has a structure of a torus. Since $\mathscr{E}_{1}=\overline{\mathbf{Q}}_{l} \otimes \mathscr{E}_{1}^{\dagger}, \mathscr{E}_{1}$ is $Z_{L}^{\iota \theta}$-equivariant local system on $\Sigma$ with respect to the action of $Z_{L}^{\iota \theta}$ on $\Sigma$ by the left multiplication. Hence $\mathscr{E}_{1} \otimes n^{*} \mathscr{E}_{1}^{\prime}$ is also $Z_{L}^{\iota \theta}$-equivariant. Since $\pi_{1}$ is $Z_{L}^{\iota \theta}$-equivariant, $\mathscr{G}$ is $Z_{L}^{\iota \theta}$ equivariant with respect to the transitive action of $Z_{L}^{\iota \theta}$ on it. Hence $\mathscr{G}$ is locally constant.

Now $\bar{\sigma}_{1}: Z_{L}^{\iota \theta} \rightarrow A_{Y}$ is a finite morphism, with $Z_{L}^{\iota \theta}$ smooth, we have, by (4.7.1), (4.8.3) $\mathscr{F}_{\mathbb{O}}$ is a perfect sheaf on $A_{Y}$.

We now show

Theorem 4.9. (i) Assume that $(L, \Sigma)$ and $\left(L^{\prime}, \Sigma^{\prime}\right)$ are not $H$-conjugate. Then $\mathscr{T}=0$.

(ii) Assume that $N$ is even, and $P=B, P^{\prime}=B_{1}, L=L^{\prime}=T$. Then $\mathscr{F}=0$.

(iii) Assume that $P=P^{\prime}, L=L^{\prime}, \Sigma=\Sigma^{\prime}$. Then $\mathscr{F}$ is a perfect sheaf on $A_{Y}$.

(iv) The natural map of sheaves $\mathscr{F} \rightarrow \widetilde{\mathscr{F}}$ is an isomorphism.

Proof. Let $E$ be a locally closed subvariety of $H / P_{H} \times H / P_{H}^{\prime}$ which is a union of some $H$-orbits, and put $Z_{0}^{E}=q^{-1}(E)$ ( $q$ is as in 4.5). One can define a constructible sheaf $\mathscr{F}_{E}$ in a similar way as in the definition of $\mathscr{F}_{\mathbb{O}}$. According to the filtration of $H / P_{H} \times H / P_{H}^{\prime}$ by various $E$, we obtain a filtration of $Z_{0}$ by various $Z_{0}^{E}$, which yields an exact sequence among various $\mathscr{F}_{E}$. In the case of $(\mathrm{i})$, since $\mathscr{F}_{\mathbb{O}}=0$ by Lemma 4.6 (i), we have $\mathscr{T}_{E}=0$ for any $E$, and so $\mathscr{T}=0$. (ii) also follows from Lemma 4.6 (iii). On the other hand, since $\mathscr{F}_{\mathbb{O}}$ is perfect by (4.8.3), by using the property on exact sequences in $(4.7 .2)$, we see that $\mathscr{F}$ is perfect. This proves (iii). 
We show (iv). By (4.5.1) we know that the map $\mathscr{F} \rightarrow \widetilde{\mathscr{F}}$ is surjective. Hence it is enough to show that the kernel of $\mathscr{F} \rightarrow \widetilde{\mathscr{F}}$ is zero. Put $\left(A_{Y}\right)_{\text {reg }}=\sigma\left(\Sigma_{\text {reg }}\right)=$ $\bar{\sigma}_{1}\left(\left(Z_{L}\right)_{\text {reg }}^{\iota \theta}\right)$. Then $\left(A_{Y}\right)_{\text {reg }}$ is an open dense subset of $A_{Y}$. Since $\mathscr{F}$ is a perfect sheaf by (i), by the property (ii) of perfect sheaves, it is enough to show that the stalk of this kernel at any point $a \in\left(A_{Y}\right)_{\text {reg }}$ is zero. So, by Lemma 4.4 (ii), we have only to show that $Z_{\beta, \beta}^{a}=\emptyset$ if $a \in\left(A_{Y}\right)_{\text {reg }}$ and if $\beta \neq \beta_{0}$ or $\beta^{\prime} \neq \beta_{0}$. Take $\left(x, g P_{H}, g^{\prime} P_{H}^{\prime}\right) \in Z$ such that the semisimple part $x_{s}$ of $x$ is $H$-conjugate to an element in $\left(Z_{L}\right)_{\text {reg }}^{\iota \theta}$ and that $g^{-1} x g \in \eta_{P}^{-1}(\bar{\Sigma}), g^{\prime-1} x g^{\prime} \in \eta_{P^{\prime}}^{-1}(\bar{\Sigma})$. Then $Z_{H}\left(x_{s}\right)^{0} \simeq L_{H}$. We must have $g^{-1} x g \in$ $\eta_{P}^{-1}(\Sigma)$ since if $g^{-1} x g \notin \eta_{P}^{-1}(\Sigma)$, then $\operatorname{dim} Z_{H}\left(g^{-1} x_{s} g\right)>\operatorname{dim} L_{H}$, a contradiction. Similarly, we have $g^{\prime-1} x g^{\prime} \in \eta_{P^{\prime}}^{-1}(\Sigma)$. This shows that $\left(x, g P_{H}, g^{\prime} P_{H}^{\prime}\right) \in Z_{\beta_{0} . \beta_{0}}^{a}$. (iv) is proved.

Proposition 4.10. Assume that $P=P^{\prime}, L=L^{\prime}, \Sigma=\Sigma^{\prime}$. Then there exists an isomorphism of sheaves on $A_{Y}$;

$$
\mathscr{F} \simeq \bigoplus_{w \in \mathscr{W}_{1}} \mathscr{F}_{\mathbb{O}(w)}
$$

where $\mathbb{O}(w)$ is an $H$-orbit corresponding to $w \in \mathscr{W}_{1} \subset \mathscr{W}=N_{H}\left(L_{H}\right) / L_{H}$.

Proof. Recall that $\mathscr{F}=R^{2 d_{0}}\left(\widetilde{\sigma}_{0}\right)_{!}\left(\overline{\mathscr{E}}_{1} \otimes \overline{\mathscr{E}}_{1}^{\prime}\right)$. Here $\widetilde{\sigma}_{0}: Z_{0} \rightarrow A_{Y}$, where $Z_{0} \simeq$ $X_{0} \times_{X} X_{0}$. By a similar argument as in the proof of [L1, Proposition 5.11], we see that $\widetilde{\sigma}_{0}^{-1}\left(\left(A_{Y}\right)_{\text {reg }}\right) \simeq \widetilde{Y} \times_{Y} \widetilde{Y}$. Thus the restriction of $\mathscr{F}$ on $\left(A_{Y}\right)_{\text {reg }}$ is the same as $R^{2 d_{0}}\left(\widetilde{\sigma}_{1}\right)_{!}\left(\widetilde{\mathscr{E}}_{1} \otimes \widetilde{\mathscr{E}}_{1}\right)$, where $\widetilde{\sigma}_{1}: \widetilde{Y} \times_{Y} \widetilde{Y} \rightarrow\left(A_{Y}\right)_{\text {reg }}$ is defined similarly to $\widetilde{\sigma}_{0}$. Consider the partition $Z_{0}=\bigcup_{\mathbb{O}} Z_{0}^{\mathbb{Q}}$ as before. The pieces $Z_{0}^{\mathbb{Q}}$ are locally closed subsets in $Z_{0}$. But since $\tilde{Y}$ is a principal $\mathscr{W}_{1}$-bundle over $Y$, the intersection $Z_{0}^{\mathbb{O}(w)} \cap\left(\widetilde{\sigma}_{0}\right)^{-1}\left(\left(A_{Y}\right)_{\text {reg }}\right)$ is an open and closed subset. Since $\mathscr{F}_{\mathbb{O}(w)}=0$ if $w \notin \mathscr{W}_{1}$ by Lemma 4.6 (ii), we have a natural isomorphism $\mathscr{F} \simeq \bigoplus_{w \in \mathscr{W}_{1}} \mathscr{F}_{\mathbb{O}(w)}$ over $\left(A_{Y}\right)_{\mathrm{reg}}$. Since $\mathscr{F}$ and $\bigoplus_{w \in \mathscr{W}_{1}} \mathscr{F}_{\mathbb{O}(w)}$ are both perfect sheaves, this isomorphism can be extended uniquely to the isomorphism over $A_{Y}$. The proposition is proved.

4.11. Under the assumption of Proposition 4.10, we consider the sheaf $\widetilde{\mathscr{F}}=$ $R^{-2 r} \widetilde{\sigma}_{!}\left(K_{\overline{\mathscr{E}}_{1}} \otimes K_{\overline{\mathscr{E}}_{1}^{\prime}}\right)$. Since $\tilde{\sigma}=\sigma \circ p_{1}$ with $p_{1}: Z=\tilde{X} \times_{X} \tilde{X} \rightarrow X$ the natural projection, one can write as

$$
\widetilde{\mathscr{T}} \simeq R^{-2 r} \sigma_{!}\left(\pi_{*} K_{\overline{\mathscr{E}}_{1}} \otimes \pi_{*} K_{\overline{\mathscr{E}}_{1}^{\prime}}\right)
$$

By Proposition $3.8, \pi_{*} K_{\overline{\mathscr{E}}_{1}} \otimes \pi_{*} K_{\overline{\mathscr{E}}_{1}^{\prime}}$ has a natural structure of a module over the algebra $\mathscr{A}_{\mathscr{E}_{1}} \otimes \mathscr{A}_{\mathscr{E}_{1}}$. Hence $\widetilde{\mathscr{T}}$ inherits a natural action of $\mathscr{A}_{\mathscr{E}_{1}} \otimes \mathscr{A}_{\mathscr{E}_{1}}$. As in [L1,3.4], $\mathscr{A}_{\mathscr{E}_{1}}$ has a natural decomposition $\mathscr{A}_{\mathscr{E}_{1}}=\bigoplus_{w \in \mathscr{W}_{\mathscr{E}_{1}}} \mathscr{A}_{\mathscr{E}_{1}, w}$ such that $\mathscr{A}_{\mathscr{E}_{1}, w} \cdot \mathscr{A}_{\mathscr{E}_{1}, w^{\prime}}=$ $\mathscr{A}_{\mathscr{E}_{1}, w w^{\prime}}$. Then the action of $\mathscr{A}_{\mathscr{E}_{1}} \otimes \mathscr{A}_{\mathscr{E}_{1}^{\prime}}$ on $\widetilde{\mathscr{T}} \simeq \mathscr{T}$ satisfies the relation (see [L1, $5.12])$

$$
\left(\mathscr{A}_{\mathscr{E}_{1}, w_{1}} \otimes \mathscr{A}_{\mathscr{E}_{1}^{\prime}, w_{1}^{\prime}}\right) \cdot \mathscr{T}_{\mathbb{O}(w)}=\mathscr{T}_{\mathbb{O}\left(w_{1} w w_{1}^{\prime-1}\right)}
$$


for $w \in \mathscr{W}_{1}, w_{1} \in \mathscr{W}_{\mathscr{E}_{1}}, w_{1}^{\prime} \in \mathscr{W}_{\mathscr{E}_{1}^{\prime}}$.

We consider the stalk $\mathscr{T}_{1}$ of $\mathscr{T}$ at $1 \in A_{Y}$. For $w \in \mathscr{W}_{1}$, the stalk $\mathscr{T}_{\mathbb{O}(w), 1}$ of $\mathscr{T}_{\mathbb{O}(w)}$ at $1 \in A_{Y}$ is given, by (4.8.1),

$$
\mathscr{T}_{\mathbb{O}(w), 1} \simeq H_{c}^{2 \operatorname{dim} \mathscr{O}_{L}}\left(\mathscr{O}_{L}, \mathscr{E}_{1} \otimes n^{*} \mathscr{E}_{1}^{\prime}\right)
$$

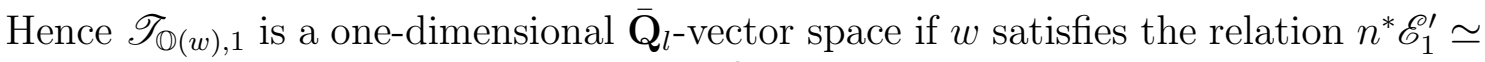
$\mathscr{E}_{1}^{*}$, the dual local system of $\mathscr{E}_{1}$, and $\mathscr{T}_{\mathbb{O}}(w), 1=0$ otherwise. Now assume that $\mathscr{E}_{1}^{\prime}=\mathscr{E}_{1}^{*}$. Then $n^{*} \mathscr{E}_{1}^{\prime} \simeq \mathscr{E}_{1}^{*}$ if and only if $w \in \mathscr{W}_{\mathscr{E}_{1}}$. Thus we have a decomposition

$$
\widetilde{\mathscr{T}_{1}}=\mathscr{T}_{1}=\bigoplus_{w \in \mathscr{W}_{\mathscr{E}_{1}}} \mathscr{T}_{\mathbb{O}}(w), 1
$$

into one-dimensional vector spaces, and (4.11.2) implies that

$$
\left(\mathscr{A}_{\mathscr{E}_{1}, w_{1}} \otimes \mathscr{A}_{\mathscr{E}_{1}, w_{1}^{\prime}}\right) \cdot \mathscr{T}_{\mathbb{O}(w), 1}=\mathscr{T}_{\mathbb{O}\left(w_{1} w w_{1}^{\prime}\right)}
$$

for $w, w_{1}, w_{1}^{\prime} \in \mathscr{W}_{\mathscr{E}_{1}}$. This is nothing but the two-sided regular representation of $\mathscr{A}_{\mathscr{E}_{1}}$ if we identify $\mathscr{A}_{\mathscr{E}_{1}^{*}}$ with $\mathscr{A}_{\mathscr{E}_{1}}^{0}$ the opposed algebra of $\mathscr{A}_{\mathscr{E}_{1}}$. Thus we have proved

Proposition 4.12. (i) $\widetilde{\mathscr{T}_{1}}=0$ if $\mathscr{E}_{1}^{\prime}$ and $\mathscr{E}_{1}^{*}$ are not conjugate under $N_{H}\left(L_{H}\right)$.

(ii) Assume that $\mathscr{E}_{1}^{\prime}=\mathscr{E}_{1}^{*}$. Then the stalk $\widetilde{\mathscr{T}_{1}}$ of $\widetilde{\mathscr{T}}$ at $1 \in A_{Y}$ is isomorphic to the $\mathscr{A}_{\mathscr{E}_{1}} \otimes \mathscr{A}_{\mathscr{E}_{1}}^{0}$-module $\mathscr{A}_{\mathscr{E}_{1}}$ (two-sided regular representation of $\mathscr{A}_{\mathscr{E}_{1}}$ ).

\section{Generalized Springer CORRESPondence}

5.1. In this section, we shall establish the generalized Springer correspondence for $G_{\text {uni }}^{\iota \theta}$ following the discussion in $[\mathrm{L} 1, \S 6]$. Let $\mathscr{N}_{G}$ be the set of pairs $(\mathscr{O}, \mathscr{E})$, where $\mathscr{O}$ is an $H$-orbit in $G_{\text {uni }}^{\iota \theta}$, and $\mathscr{E}$ is an $H$-equivariant simple local system on $\mathscr{O}$. We consider a triple $\left(L \subset P, \mathscr{O}_{L}, \mathscr{E}_{1}^{\dagger}\right)$, where $L$ is a $\theta$-stable Levi subgroup of a $\theta$-stable parabolic subgroup $P$ of $G$, and $\mathscr{O}_{L}$ is an $L_{H^{-}}$orbit in $L_{\text {uni }}^{\iota \theta}, \mathscr{E}_{1}^{\dagger}$ is an $L_{H^{-}}$-equivariant simple local system on $\mathscr{O}_{L}$. Put $\Sigma=Z_{L}^{\iota \theta} \mathscr{O}_{L}$, and $\mathscr{E}_{1}=\overline{\mathbf{Q}}_{l} \otimes \mathscr{E}_{1}^{\dagger}$. Let $\mathscr{S}_{G}$ be the set of triples $\left(L \subset P, \mathscr{O}_{L}, \mathscr{E}_{1}^{\dagger}\right)$, up to $H$-conjugate, such that $\mathscr{E}_{1}$ is cuspidal on $\Sigma$. (Note that in the case where $N$ is odd, $P$ is determined by L, up to $H$-conjugate, hence we need not to write $P$ in the notation of $\mathscr{S}_{G}$ (see $[\mathrm{L} 1, \S 6]$ ). But in the case where $N$ is even and $L=T$, we need to distinguish the two cases $T \subset B$ and $T \subset B_{1}$ ).

For each $\left(L \subset P, \mathscr{O}_{L}, \mathscr{E}_{1}^{\dagger}\right) \in \mathscr{S}_{G}$, one can construct a semisimple perverse sheaf $K=\pi_{*} K_{\overline{\mathscr{E}}_{1}}$ on $X=\bar{Y}$ with $Y=Y_{(L, \Sigma)}$. By Proposition 3.8, $K$ is equipped with an action of $\mathscr{A}_{\mathscr{E}_{1}}$, and is decomposed into simple perverse sheaves as follows;

$$
K \simeq \bigoplus_{\rho \in \mathscr{A}_{\mathscr{E}_{1}}} \rho \otimes K_{\rho}
$$


where $K_{\rho}=\operatorname{Hom}(\rho, K)$. We consider the restriction $\left.K\right|_{G_{\text {uni }}^{\iota \theta}}$ of $K$ on $G_{\text {uni }}^{\iota \theta}$. Then $\left.K\right|_{G_{\text {uni }}^{\iota \theta}}$ inherits the natural action of $\mathscr{A}_{\mathscr{E}_{1}}$. The following result gives the generalized Springer correspondence for $G_{\mathrm{uni}}^{\iota \theta}$, which is an analogue of [L1, Theorem 6.5]

Theorem 5.2 (generalized Springer correspondence). Under the notation above,

(i) Let $K=\pi_{*} K_{\overline{\mathscr{E}} 1}$ for $\left(L \subset P, \mathscr{O}_{L}, \mathscr{E}_{1}^{\dagger}\right) \in \mathscr{S}_{G}$, and put $r=\operatorname{dim} Z_{L}^{\iota \theta}$. Then $\left.K[-r]\right|_{X_{\mathrm{uni}}}$ is a semisimple perverse sheaf on $X_{\mathrm{uni}}=X \cap G_{\mathrm{uni}}^{\iota \theta}$, and is decomposed into simple perverse sheaves,

$$
\left.K[-r]\right|_{X_{\mathrm{uni}}} \simeq \bigoplus_{(\mathscr{O}, \mathscr{E}) \in \mathscr{N}_{G}} V_{(\mathscr{O}, \mathscr{E})} \otimes \mathrm{IC}(\overline{\mathscr{O}}, \mathscr{E})[\operatorname{dim} \mathscr{O}]
$$

The action of $\mathscr{A}_{\mathscr{E} 1}$ on $\left.K[-r]\right|_{G_{\mathrm{uni}} \mathrm{\theta}}$ induces an action of $\mathscr{A}_{\mathscr{E}_{1}}$ on $V_{(\mathscr{O}, \mathscr{E})}$, which makes $V_{(\mathscr{O}, \mathscr{E})}$ an irreducible $\mathscr{A}_{\mathscr{E} 1}$ module if it is non-zero. The map $(\mathscr{O}, \mathscr{E}) \mapsto$ $V_{(\mathscr{O}, \mathscr{E})}$ gives a bijection

$$
\left\{(\mathscr{O}, \mathscr{E}) \in \mathscr{N}_{G} \mid V_{(\mathscr{O}, \mathscr{E})} \neq 0\right\} \simeq \mathscr{A}_{\mathscr{E} 1}^{\wedge}
$$

Moreover, under the above correspondence $(\mathscr{O}, \mathscr{E}) \leftrightarrow \rho$, we have

$$
\left.K_{\rho}[-\operatorname{dim} X]\right|_{X_{\text {uni }}} \simeq \operatorname{IC}(\overline{\mathscr{O}}, \mathscr{E})\left[-2 d_{\mathscr{O}}\right]
$$

where we put $d_{\mathscr{O}}=\left(\nu_{H}-\operatorname{dim} \mathscr{O} / 2\right)-\left(\nu_{L_{H}}-\operatorname{dim} \mathscr{O}_{L} / 2\right)+\Delta_{P} / 2$.

(ii) For each $(\mathscr{O}, \mathscr{E}) \in \mathscr{N}_{G}$, there exists a unique $\left(L \subset P, \mathscr{O}_{L}, \mathscr{E}_{1}^{\dagger}\right) \in \mathscr{S}_{G}$ such that $\mathrm{IC}(\overline{\mathscr{O}}, \mathscr{E})[\operatorname{dim} \mathscr{O}]$ is a direct summand of $\pi_{*} K_{\overline{\mathscr{E}}_{1}}$. The correspondence $(\mathscr{O}, \mathscr{E}) \mapsto V_{(\mathscr{O}, \mathscr{E})}$ gives a bijection

$$
\mathscr{N}_{G} \simeq \coprod_{\left(L \subset P, \mathscr{O}_{L}, \mathscr{E}_{1}^{\dagger}\right) \in \mathscr{S}_{G}} \mathscr{A}_{\mathscr{E}_{1}}^{\wedge}
$$

(iii) For $\left(L \subset P, \mathscr{O}_{L}, \mathscr{E}_{1}^{\dagger}\right) \in \mathscr{S}_{G}$, let $f: \widetilde{X}_{0} \rightarrow X$ be the restriction of $\pi: \tilde{X} \rightarrow X$. Under the correspondence in (ii), the condition $(\mathscr{O}, \mathscr{E})$ corresponds to $(L \subset$ $\left.P, \mathscr{O}_{L}, \mathscr{E}_{1}^{\dagger}\right)$ is that $\overline{\mathscr{O}} \subset X$ and that $\mathscr{E}$ appears as a direct summand in the local system $\left.R^{2 d_{\mathscr{O}}} f_{!} \overline{\mathscr{E}}_{1}\right|_{\mathscr{O}}$. Moreover, in that case, the natural homomorphism, obtained from the embedding $\tilde{X}_{0} \subset \tilde{X}$,

$$
\left.\left.R^{2 d_{\mathscr{O}}} f_{!} \overline{\mathscr{E}}_{1}\right|_{\mathscr{O}} \rightarrow \mathscr{H}^{2 d_{\mathscr{O}}}\left(\pi_{*} K_{\overline{\mathscr{E}}_{1}}[-\operatorname{dim} X]\right)\right|_{\mathscr{O}}
$$

is an isomorphism.

5.3. The remainder of this section is devoted to the proof of the theorem. Let $P=L U_{P}$ be a $\theta$-stable parabolic subgroup of $G$, with $\theta$-stable Levi subgroup $L$. Let $\mathscr{O}$ be an $H$-orbit in $G_{\text {uni }}^{\iota \theta}, \mathscr{O}_{L}$ an $L_{H^{-}}$orbit in $L_{\text {uni }}^{\iota \theta}$. We consider the following diagram. 


$$
\begin{gathered}
V=H \times{ }^{P_{H}}\left(\mathscr{O} \cap \eta_{P}^{-1}\left(\mathscr{O}_{L}\right)\right) \stackrel{f_{1}}{\longrightarrow} \mathscr{O} \\
f_{2} \downarrow \\
V^{\prime}=H \times{ }^{P_{H}} \mathscr{O}_{L} .
\end{gathered}
$$

Here in $V^{\prime}$, we consider the action of $P_{H}$ on $\mathscr{O}_{L}$ such that $U_{P_{H}}$ acts trivially. $f_{1}$ is the map induced from the map $(g, x) \mapsto g x g^{-1}$, and $f_{2}$ is the map induced from the map $\mathscr{O} \cap \eta_{P}^{-1}\left(\mathscr{O}_{L}\right) \rightarrow \mathscr{O}_{L}$ which is the restriction of the projection $\eta_{P}: P^{\iota \theta} \rightarrow L^{\iota \theta}$. $H$ acts on $V, V^{\prime}, \mathscr{O}$ naturally, and $f_{1}, f_{2}$ are $H$-equivariant. Moreover, the action of $H$ on $V^{\prime}$ and on $\mathscr{O}$ are transitive. Put

$$
\begin{aligned}
& d_{1}=\left(\nu_{H}-\operatorname{dim} \mathscr{O} / 2\right)-\left(\nu_{L_{H}}-\operatorname{dim} \mathscr{O}_{L} / 2\right)+\Delta_{P} / 2, \\
& d_{2}=\left(\operatorname{dim} \mathscr{O}-\operatorname{dim} \mathscr{O}_{L}\right) / 2+\Delta_{P} / 2
\end{aligned}
$$

By Proposition 2.2 (ii), (i), all the fibres of $f_{1}$ have dimension $\leq d_{1}$ and all the fibres of $f_{2}$ have dimension $\leq d_{2}$. Moreover, some (or all) fibre of $f_{1}$ has dimension $d_{1}$ if and only if some (or all) fibre of $f_{2}$ has dimension $d_{2}$ since this is equivalent to the condition that $\operatorname{dim} V=\left(\nu_{H}-\nu_{L_{H}}\right)+\left(\operatorname{dim} \mathscr{O}+\operatorname{dim} \mathscr{O}_{L}\right) / 2+\Delta_{P} / 2$. Under this situation, the following result was proved by [L1, (6.1.1)].

Lemma 5.4. Let $\mathscr{F}$ be an $H$-equivariant simple local system on $\mathscr{O}$, and $\mathscr{F}^{\prime}$ be an $H$-equivariant simple local system on $V^{\prime}$ (note that $V^{\prime}$ is a single $H$-orbit). Then the multiplicity of $\mathscr{F}$ in the $H$-equivariant local system $R^{2 d_{1}}\left(f_{1}\right)_{!}\left(f_{2}^{*} \mathscr{F}^{\prime}\right)$ on $\mathscr{O}$ is equal to the multiplicity of $\mathscr{F}^{\prime}$ in the $H$-equivariant local system $R^{2 d_{2}}\left(f_{2}\right) !\left(f_{1}^{*} \mathscr{F}\right)$ on $V^{\prime}$.

5.5. If $(\mathscr{O}, \mathscr{E}) \in \mathscr{N}_{G}$ is a cuspidal pair, by Lemma 2.5 , for any $\theta$-stable parabolic subgroup $P \neq G$, and any $L_{H}$-orbit $\mathscr{O}_{L}$ of $L_{\text {uni }}^{\iota \theta}$, we have

$$
H_{c}^{2 \delta}\left(\mathscr{O} \cap \eta_{P}^{-1}(v), \mathscr{E}\right)=0
$$

where $\delta=\left(\operatorname{dim} \mathscr{O}-\operatorname{dim} \mathscr{O}_{L}\right) / 2+\Delta_{P} / 2$ and $v \in \mathscr{O}_{L}$. Now let $(\mathscr{O}, \mathscr{E}) \in \mathscr{N}_{G}$ be an arbitrary element. One can find a $\theta$-stable parabolic subgroup $P$ of $G$ with the $\theta$-stable Levi subgroup $L$ and an $L_{H^{-}}$orbit $\mathscr{O}_{L}$ in $L_{\text {uni }}^{\iota \theta}$ satisfying the property

$$
H_{c}^{2 \delta}\left(\mathscr{O} \cap \eta_{P}^{-1}(v), \mathscr{E}\right) \neq 0
$$

for $v \in \mathscr{O}_{L}$. We choose $P$ a $\theta$-stable minimal parabolic subgroup of $G$ satisfying the property (5.5.1). Note that $P=G$ satisfies the condition (5.5.1).

Let $f_{2}: \mathscr{O} \cap \eta_{P}^{-1}\left(\mathscr{O}_{L}\right) \rightarrow \mathscr{O}_{L}$ be the map defined by $x \mapsto \eta_{P}(x)$, and let $\mathscr{E}_{1}^{\dagger}$ be a $L_{H^{-}}$equivariant simple local system on $\mathscr{O}_{L}$ which is a direct summand of $R^{2 \delta}\left(f_{2}\right)_{!}(\mathscr{E})$.

Proposition 5.6. The triple $\left(L \subset P, \mathscr{O}_{L}, \mathscr{E}_{1}^{\dagger}\right)$ is uniquely determined by $(\mathscr{O}, \mathscr{E})$. Moreover, $\left(\mathscr{O}_{L}, \mathscr{E}_{1}^{\dagger}\right)$ is a cuspidal pair on $L^{\iota \theta}$, namely $\left(L \subset P, \mathscr{O}_{L}, \mathscr{E}_{1}^{\dagger}\right) \in \mathscr{S}_{G}$. 
Proof. Suppose that $\left(\mathscr{O}_{L}, \mathscr{E}_{1}^{\dagger}\right)$ is not cuspidal. Then there exists a $\theta$-stable parabolic subgroup $P^{\prime} \subsetneq P$ and a Levi subgroup $L^{\prime}$ of $P^{\prime}$ such that $L^{\prime} \subset L$, and an $L_{H^{-}}^{\prime}$-orbit $\mathscr{O}_{L^{\prime}}$ in $L_{\text {uni }}^{\prime \prime \theta}$ satisfying the property

$$
H_{c}^{2 \delta^{\prime}}\left(\mathscr{O}_{L} \cap \eta_{L \cap P^{\prime}}^{-1}\left(v^{\prime}\right), \mathscr{E}_{1}^{\dagger}\right) \neq 0
$$

for $v^{\prime} \in \mathscr{O}_{L^{\prime}}$, where $\delta^{\prime}=\left(\operatorname{dim} \mathscr{O}_{L}-\operatorname{dim} \mathscr{O}_{L^{\prime}}\right) / 2+\Delta_{L \cap P^{\prime}}$. It follows that

$$
H_{c}^{2 \delta^{\prime}}\left(\mathscr{O}_{L} \cap \eta_{L \cap P^{\prime}}^{-1}\left(v^{\prime}\right), R^{2 \delta}\left(f_{2}\right) !(\mathscr{E})\right) \neq 0 .
$$

The map $f_{2}$ defines a map $\mathscr{O} \cap \eta_{P^{\prime}}^{-1}\left(v^{\prime}\right) \mapsto \mathscr{O}_{L} \cap \eta_{L \cap P^{\prime}}^{-1}\left(v^{\prime}\right)$ by the restriction, and all the fibres have dimension $\leq \delta$ by Proposition 2.2. By the Leray spectral sequence, we have

$$
H_{c}^{2 \delta^{\prime}+2 \delta}\left(\mathscr{O} \cap \eta_{P^{\prime}}^{-1}\left(v^{\prime}\right), \mathscr{E}\right) \neq 0
$$

Since $\Delta_{P}+\Delta_{L \cap P^{\prime}}=\Delta_{P^{\prime}}$, we have $\delta^{\prime}+\delta=\left(\operatorname{dim} \mathscr{O}-\operatorname{dim} \mathscr{O}_{L^{\prime}}\right) / 2+\Delta_{P^{\prime}}$. This contradicts the minimality of $P$. Hence $\mathscr{E}_{1}^{\dagger}$ is cuspidal on $\mathscr{O}_{L}$.

Let $\Sigma=Z_{L}^{\iota \theta} \mathscr{O}_{L}$, and put $\mathscr{E}_{1}=\overline{\mathbf{Q}}_{l} \otimes \mathscr{E}_{1}^{\dagger}$. Then $\left(\Sigma, \mathscr{E}_{1}\right)$ is a cuspidal pair in $L$. By Lemma 2.7, $L_{H}$ is of the form $L_{H} \simeq\left(G L_{1}\right)^{a} \times S O_{N_{0}}$. Hence the discussion in Section 3 can be applied. We consider $Y=Y_{(L, \Sigma)}$ and the map $\pi: \widetilde{X} \rightarrow X=\bar{Y}$. Let $\widetilde{X}_{0} \simeq H \times{ }^{P_{H}} \eta_{P}^{-1}(\Sigma)$ be as in 3.7. The local system $\overline{\mathscr{E}}_{1}$ on $\widetilde{X}_{0}$ is constructed from $\mathscr{E}_{1}$. Let $f: \widetilde{X}_{0} \rightarrow X$ be the restriction of the map $\pi: \widetilde{X} \rightarrow X$. Then the restriction of $f$ on $f^{-1}(\mathscr{O})$ coincides with $f_{1}$ in 5.3. By the definition of $(L \subset$ $\left.P, \mathscr{O}_{L}, \mathscr{E}_{1}^{\dagger}\right)$ and by Lemma 5.4 , we see that $\mathscr{E}$ is a direct summand of $\left.R^{2 d} f_{!}\left(\overline{\mathscr{E}}_{1}\right)\right|_{\mathscr{O}}$, where $d=\left(\nu_{H}-\operatorname{dim} \mathscr{O} / 2\right)-\left(\nu_{L_{H}}-\operatorname{dim} \mathscr{O}_{L} / 2\right)+\Delta_{P} / 2\left(\right.$ note that $d_{1}=\delta, d_{2}=d$ in the notation in Lemma 5.4).

We show that such a triple $\left(L \subset P, \mathscr{O}_{L}, \mathscr{E}_{1}^{\dagger}\right) \in \mathscr{S}_{G}$ is uniquely determined from $(\mathscr{O}, \mathscr{E})$. Suppose that there exists another $\theta$-stable minimal parabolic subgroup $P^{\prime}$ satisfying (5.5.1), and $\left(L^{\prime} \subset P^{\prime}, \mathscr{O}_{L^{\prime}}, \mathscr{E}_{1}^{\prime \dagger}\right) \in \mathscr{S}_{G}$. We can define a map $f^{\prime}: \widetilde{X}_{0}^{\prime} \rightarrow X^{\prime}$ and a local system $\overline{\mathscr{E}}_{1}^{\prime}$ on $\widetilde{X}_{0}^{\prime}$ as before. We see that $\mathscr{E}$ appears as a direct summand in $\left.R^{2 d^{\prime}} f_{!}^{\prime}\left(\overline{\mathscr{E}}_{1}^{\prime}\right)\right|_{\mathscr{O}}$. It follows that $\left.R^{2 d} f_{!}\left(\overline{\mathscr{E}}_{1}\right) \otimes R^{2 d^{\prime}} f_{1}^{\prime}\left(\overline{\mathscr{E}}_{1}^{\prime *}\right)\right|_{\mathscr{O}}$ contains a constant sheaf $\overline{\mathrm{Q}}_{l}$ on $\mathscr{O}$, where $\overline{\mathscr{E}}^{\prime *}$ is the dual local system of $\overline{\mathscr{E}}_{1}^{\prime}$. Hence

$$
H_{c}^{2 \operatorname{dim} \mathscr{O}}\left(\mathscr{O},\left.R^{2 d} f_{!}\left(\overline{\mathscr{E}}_{1}\right) \otimes R^{2 d^{\prime}} f_{!}^{\prime}\left(\overline{\mathscr{E}}_{1}^{\prime *}\right)\right|_{\mathscr{O}}\right) \neq 0
$$

Let $p: Z_{0}=\widetilde{X}_{0} \times_{G^{\iota \theta}} \tilde{X}_{0}^{\prime} \rightarrow G^{\iota \theta}$ be the natural projection, and put $Z_{0, \mathscr{O}}=p^{-1}(\mathscr{O})$. Since for each $x \in \mathscr{O}$, $\operatorname{dim} p^{-1}(x) \leq d+d^{\prime}$, (5.6.1) implies, by the Leray spectral sequence, that

$$
H_{c}^{2 d_{0}}\left(Z_{0, \mathscr{O}}, \overline{\mathscr{E}}_{1} \otimes \overline{\mathscr{E}}_{1}^{*}\right) \neq 0
$$

where $d_{0}=d+d^{\prime}+\operatorname{dim} \mathscr{O}$ is as in (4.3.1). We consider $Z_{0}^{1}=Z_{\beta_{0}, \beta_{0}^{\prime}}^{1}$ for $a=1 \in$ $A_{Y} \cap A_{Y^{\prime}}$ under the notation in 4.3. Then $Z_{0, \mathscr{O}^{\prime}}$ form a partition of $Z_{0}^{1}$ by locally 
closed pieces $Z_{0, \mathscr{O}^{\prime}}$ of dimension $\leq d_{0}$ (by Lemma 4.4) if $\mathscr{O}^{\prime}$ varies the $H$-orbits in $G_{\text {uni }}^{\iota \theta}$ It follows from (5.6.2), that

$$
H_{c}^{2 d_{0}}\left(Z_{0}^{1}, \overline{\mathscr{E}}_{1} \otimes \overline{\mathscr{E}}_{1}^{\prime *}\right) \neq 0
$$

This means that $\mathscr{T}_{1} \neq 0$, where $\mathscr{T}$ is defined with respect to $\left(L \subset P, \Sigma, \mathscr{E}_{1}\right)$ and $\left(L^{\prime} \subset P^{\prime}, \Sigma^{\prime}, \mathscr{E}_{1}^{\prime *}\right)$. Then by Theorem 4.9, $P=P^{\prime}, L=L^{\prime}, \Sigma=\Sigma^{\prime}$. Moreover by Proposition $4.12, \mathscr{E}_{1}^{\prime}$ is $N_{H}\left(L_{H}\right)$-conjugate to $\mathscr{E}_{1}$. Thus the triple $\left(L \subset P, \mathscr{O}_{L}, \mathscr{E}_{1}^{\dagger}\right)$ is uniquely determined from $(\mathscr{O}, \mathscr{E})$.

5.7. By Proposition 5.6, for each $(\mathscr{O}, \mathscr{E}) \in \mathscr{N}_{G}$, there exists a unique triple $\left(L \subset P, \mathscr{O}_{L}, \mathscr{E}_{1}^{\dagger}\right) \in \mathscr{S}_{G}$. In this case, we say that $(\mathscr{O}, \mathscr{E})$ belongs to the series $\left(L \subset P, \mathscr{O}_{L}, \mathscr{E}_{1}^{\dagger}\right)$. Hence we have a partition

$$
\mathscr{N}_{G}=\coprod_{\xi \in \mathscr{S}_{G}} \mathscr{N}_{G}^{(\xi)}
$$

where $\mathscr{N}_{G}^{(\xi)}$ is the set of all $(\mathscr{O}, \mathscr{E})$ which belong to the series $\xi=\left(L \subset P, \mathscr{O}_{L}, \mathscr{E}_{1}^{\dagger}\right)$.

For each $\left(L \subset P, \mathscr{O}_{L}, \mathscr{E}_{1}^{\dagger}\right) \in \mathscr{S}_{G}$, we consider $Y=Y_{(L, \Sigma)}$ and $X=\bar{Y}$. Let $f: \widetilde{X}_{0} \rightarrow X$ be the restriction of $\pi: \tilde{X} \rightarrow X$. The following result gives a characterization of the set $\mathscr{N}_{G}^{(\xi)}$.

Lemma 5.8. $(\mathscr{O}, \mathscr{E}) \in \mathscr{N}_{G}$ belong to $\xi=\left(L \subset P, \mathscr{O}_{L}, \mathscr{E}_{1}^{\dagger}\right)$ if and only if $\mathscr{O} \subset X$ and $\mathscr{E}$ is a direct summand of $\left.R^{2 d} f_{!}\left(\overline{\mathscr{E}}_{1}\right)\right|_{\mathscr{O}}$, where $d=\left(\nu_{H}-\operatorname{dim} \mathscr{O} / 2\right)-\left(\nu_{L_{H}}-\right.$ $\left.\operatorname{dim} \mathscr{O}_{L} / 2\right)+\Delta_{P} / 2$.

Proof. In the course of the proof of Proposition 5.6, we have already shown that if $(\mathscr{O}, \mathscr{E})$ belongs to $\left(L \subset P, \mathscr{O}_{L}, \mathscr{E}_{1}^{\dagger}\right)$, then $\mathscr{O} \subset X$, and $\mathscr{E}$ is a direct summand of $\left.R^{2 d} f_{1}\left(\overline{\mathscr{E}}_{1}\right)\right|_{\mathscr{O}}$. Conversely, assume that $\mathscr{O} \subset X$ and $\mathscr{E}$ is a direct summand of $\left.R^{2 d} f_{!}\left(\overline{\mathscr{E}}_{1}\right)\right|_{\mathscr{O}}$. Then by Lemma $5.4, \mathscr{E}_{1}^{\dagger}$ is a direct summand of $R^{2 \delta}\left(f_{2}\right) !(\mathscr{E})$, where $\delta$ and $f_{2}$ are defined as in 5.5. The discussion in the proof of Proposition 5.6 shows that $\left(P, L, \mathscr{O}_{L}\right)$ satisfies the condition in $(5.5 .1)$, and if such a choice of $\left(P, L, \mathscr{O}_{L}\right)$ is not minimal, it contradicts that $\left(\mathscr{O}_{L}, \mathscr{E}_{1}^{\dagger}\right)$ is a cuspidal pair. Hence $(\mathscr{O}, \mathscr{E})$ belongs to $\left(L \subset P, \mathscr{O}_{L}, \mathscr{E}_{1}^{\dagger}\right)$ Thus

5.9. We fix $\left(L \subset P, \mathscr{O}_{L}, \mathscr{E}_{1}^{\dagger}\right) \in \mathscr{S}_{G}$. Put $X_{\text {uni }}=X \cap G_{\text {uni }}^{\iota \theta}$, and $\widetilde{X}_{\text {uni }}=\pi^{-1}\left(X_{\text {uni }}\right)$.

$$
\begin{aligned}
\tilde{X}_{\text {uni }} & =\left\{\left(x, g P_{H}\right) \in G^{\iota \theta} \times H / P_{H} \mid g^{-1} x g \in \eta_{P}^{-1}\left(\overline{\mathscr{O}}_{L}\right)\right\} \\
X_{\text {uni }} & =\bigcup_{g \in H} g\left(\eta_{P}^{-1}\left(\overline{\mathscr{O}}_{L}\right)\right) g .
\end{aligned}
$$

Let $\pi_{1}: \widetilde{X}_{\text {uni }} \rightarrow X_{\text {uni }}$ be the restriction of $\pi$ on $\widetilde{X}_{\text {uni }}$. Since $\pi_{1}$ is proper, surjective, $X_{\text {uni }}$ is a closed subset of $G_{\text {uni }}^{\iota \theta}$. 
Lemma 5.10. $\widetilde{X}_{\text {uni }}, X_{\text {uni }}$ are irreducible varieties. We have

$$
\operatorname{dim} \widetilde{X}_{\text {uni }}=\operatorname{dim} X_{\text {uni }}=2 \nu_{H}-2 \nu_{L_{H}}+\operatorname{dim} \mathscr{O}_{L}+\Delta_{P}
$$

Proof. Since $\widetilde{X}_{\text {uni }} \simeq H \times{ }^{P_{H}} \eta_{P}^{-1}\left(\overline{\mathscr{O}}_{L}\right), \widetilde{X}_{\text {uni }}$ is irreducible. Also $\operatorname{dim} \widetilde{X}_{\text {uni }}=\operatorname{dim} H / P_{H}+$ $\operatorname{dim} \eta_{P}^{-1}\left(\overline{\mathscr{O}}_{L}\right)=2 \nu_{H}-2 \nu_{L_{H}}+\operatorname{dim} \mathscr{O}_{L}+\Delta_{P}$ (compare with Lemma 3.5). Thus $\operatorname{dim} \widetilde{X}_{\text {uni }}=d_{0}$. Since $\pi_{1}$ is surjective, $\operatorname{dim} \widetilde{X}_{\text {uni }} \geq \operatorname{dim} X_{\text {uni }}$, and $X_{\text {uni }}$ is irreducible. Suppose that $\operatorname{dim} X_{\text {uni }}=\delta<\operatorname{dim} \widetilde{X}_{\text {uni }}$. Then there exists an open dense subset $D$ of $X_{\text {uni }}$ such that $\operatorname{dim} \pi_{1}^{-1}(x)=\operatorname{dim} \tilde{X}_{\text {uni }}-\delta$ for $x \in D$. We consider the fibre product $\pi_{1}^{-1}(D) \times_{D} \pi_{1}^{-1}(D)$. It has the dimension $\operatorname{dim} D+2\left(\operatorname{dim} \widetilde{X}_{\text {uni }}-\delta\right)=2 \operatorname{dim} \widetilde{X}_{\text {uni }}-\delta$. On the other hand, $\pi_{1}^{-1}(D) \times_{D} \pi_{1}^{-1}(D) \subset \widetilde{X}_{\text {uni }} \times_{X_{\text {uni }}} \widetilde{X}_{\text {uni }} \simeq Z^{\prime}$, where $Z^{\prime}$ is as in 2.1. By Proposition 2.2 (iv), we have $\operatorname{dim} Z^{\prime} \leq d_{0}=\operatorname{dim} \tilde{X}_{\text {uni }}$. It follows that $\operatorname{dim} \widetilde{X}_{\text {uni }} \geq 2 \operatorname{dim} \widetilde{X}_{\text {uni }}-\delta$, and so $\delta \geq \operatorname{dim} \widetilde{X}_{\text {uni }}$. This is a contradiction, and the lemma follows.

5.11. We now consider the restriction of $\pi_{*} K_{\overline{\mathscr{E}}_{1}}$ on $X_{\text {uni }}$. Since $\bar{\Sigma} \simeq Z_{L}^{\iota \theta} \times \overline{\mathscr{O}}_{L}$, $\mathrm{IC}\left(\bar{\Sigma}, \mathscr{E}_{1}\right) \simeq \overline{\mathrm{Q}}_{l} \otimes \mathrm{IC}\left(\overline{\mathscr{O}}_{L}, \mathscr{E}_{1}^{\dagger}\right)$. It follows that the restriction of $K_{\overline{\mathscr{E}}_{1}}=\operatorname{IC}\left(\widetilde{X}, \overline{\mathscr{E}}_{1}\right)[\operatorname{dim} \tilde{X}]$ on $\widetilde{X}_{\text {uni }}$ coincides with $K \frac{1}{\mathscr{E}_{1}}[r]$, where $K_{\overline{\mathscr{E}}_{1}}=\mathrm{IC}\left(\widetilde{X}_{\text {uni }}, \overline{\mathscr{E}}_{1}^{\dagger}\right)\left[\operatorname{dim} \widetilde{X}_{\text {uni }}\right]$. Here $\overline{\mathscr{E}}_{1}^{\dagger}$ is defined from $\mathscr{E}_{1}^{\dagger}$ in a similar way as $\overline{\mathscr{E}}_{1}$ is defined from $\mathscr{E}_{1}$. (Note that $\operatorname{dim} \tilde{X}-$ $\operatorname{dim} \widetilde{X}_{\text {uni }}=r$ by Lemma 5.10 and (3.2.1).) Hence the restriction of $\pi_{*} K_{\overline{\mathscr{E}}_{1}}$ on $X_{\text {uni }}$ coincides with $\left(\pi_{1}\right)_{*} K_{\mathscr{E}_{1}}[r]$. The following lemma can be proved in a similar way as Proposition 3.8, by using Proposition 2.2 (iv) (see also [L1, 6.6]).

Lemma 5.12. $\left.\pi_{*} K_{\overline{\mathscr{E}}_{1}}[-r]\right|_{X_{\mathrm{uni}}} \simeq\left(\pi_{1}\right)_{*} K_{\mathscr{E}_{1}}$ is a perverse sheaf on $X_{\text {uni }}$.

5.13. Since $\pi_{1}$ is proper, by Deligne-Gabber's decomposition theorem, $\left(\pi_{1}\right)_{*} K_{\mathscr{E}_{1}}$ is a semisimple perverse sheaf on $X_{\text {uni }}$. Since $\pi_{1}$ is $H$-equivariant, each simple component in $\left(\pi_{1}\right)_{*} K_{\mathscr{E}} \frac{1}{1}$ is an $H$-equivariant simple perverse sheaf, hence it is of the form $K(\mathscr{E})=\operatorname{IC}(\overline{\mathscr{O}}, \mathscr{E})[\operatorname{dim} \mathscr{O}]$ for some $(\mathscr{O}, \mathscr{E}) \in \mathscr{N}_{G}$. Here we prepare a lemma.

Lemma 5.14. Take $(\mathscr{O}, \mathscr{E}),\left(\mathscr{O}^{\prime}, \mathscr{E}^{\prime}\right) \in \mathscr{N}_{G}$, and assume that $\mathscr{O}, \mathscr{O}^{\prime} \subset X_{\text {uni }}$. Then

$$
\operatorname{dim} \mathbf{H}_{c}^{0}\left(X_{\text {uni }}, K(\mathscr{E}) \otimes K\left(\mathscr{E}^{\prime \prime}\right)\right)= \begin{cases}1 & \text { if } \mathscr{O}=\mathscr{O}^{\prime} \text { and } \mathscr{E}^{\prime} \simeq \mathscr{E}^{*} \\ 0 & \text { otherwise. }\end{cases}
$$

Proof. First, by assuming that $\mathscr{O} \neq \mathscr{O}^{\prime}$, we show that

$$
\mathbf{H}_{c}^{0}\left(X_{\text {uni }}, K(\mathscr{E}) \otimes K\left(\mathscr{E}^{\prime}\right)\right)=0
$$

In order to show (5.14.1), it is enough to see, by using the hypercohomology spectral sequence, that

$$
\text { If } H_{c}^{i}\left(X_{\text {uni }}, \mathscr{H}^{j} K(\mathscr{E}) \otimes \mathscr{H}^{j^{\prime}} K\left(\mathscr{E}^{\prime}\right)\right) \neq 0 \text {, then } i+j+j^{\prime}<0 \text {. }
$$


Put $D_{j, j^{\prime}}=\operatorname{supp} \mathscr{H}^{j} K(\mathscr{E}) \cap \operatorname{supp} \mathscr{H}^{j^{\prime}} K\left(\mathscr{E}^{\prime}\right)$. Since the cohomology in $(5.14 .2)$ is not changed if we replace $X_{\text {uni }}$ by $D_{j, j^{\prime}}$. Thus we may assume that

$$
H_{c}^{i}\left(D_{j, j^{\prime}}, \mathscr{H}^{j} K(\mathscr{E}) \otimes \mathscr{H}^{j^{\prime}} K\left(\mathscr{E}^{\prime}\right)\right) \neq 0
$$

It follows that $i \leq 2 \operatorname{dim} D_{j, j^{\prime}}$. By the condition on the intersection cohomology for $K(\mathscr{E})$ and $K\left(\mathscr{E}^{\prime}\right)$, we have

$$
\begin{aligned}
& \operatorname{dim} D_{j, j^{\prime}} \leq \operatorname{dim} \operatorname{supp} \mathscr{H}^{j} K(\mathscr{E}) \leq-j \\
& \operatorname{dim} D_{j, j^{\prime}} \leq \operatorname{dim} \operatorname{supp} \mathscr{H}^{j^{\prime}} K\left(\mathscr{E}^{\prime}\right) \leq-j^{\prime} .
\end{aligned}
$$

Hence $j, j^{\prime} \leq-\operatorname{dim} D_{j, j^{\prime}}$. If we assume both are equalities, then we have $\mathscr{O}=$ $D_{j, j^{\prime}}=\mathscr{O}^{\prime}$, a contradiction. Hence we have $i+j+j^{\prime}<0$, as asserted.

Next assume that $\mathscr{O}=\mathscr{O}^{\prime}$. Put $D=\overline{\mathscr{O}}-\mathscr{O}$. We show the following.

$$
\mathbf{H}_{c}^{0}\left(D, K(\mathscr{E}) \otimes K\left(\mathscr{E}^{\prime}\right)\right)=0 \text { and } \mathbf{H}_{c}^{-1}\left(D, K(\mathscr{E}) \otimes K\left(\mathscr{E}^{\prime}\right)\right)=0 .
$$

As in the previous argument, we consider the condition that

$$
H_{c}^{i}\left(D_{j, j^{\prime}}, \mathscr{H}^{j} K(\mathscr{E}) \otimes \mathscr{H}^{j^{\prime}} K\left(\mathscr{E}^{\prime}\right)\right) \neq 0
$$

where $D_{j, j^{\prime}}=D \cap \operatorname{supp} \mathscr{H}^{j} K(\mathscr{E}) \cap \operatorname{supp} \mathscr{H}^{j^{\prime}} K\left(\mathscr{E}^{\prime}\right)$. Again we have $i \leq 2 \operatorname{dim} D_{j, j^{\prime}}$. But since $\operatorname{dim} D<\operatorname{dim} \mathscr{O}$, we have inequalities $j<-\operatorname{dim} D_{j, j^{\prime}}, j^{\prime}<-\operatorname{dim} D_{j, j^{\prime}}$, and so $i+j+j^{\prime}<-1$. This proves (5.14.3). By using the cohomology long exact sequence with respect to $\mathscr{O} \subset \overline{\mathscr{O}},(5.14 .3)$ implies that

$$
\mathbf{H}_{c}^{0}\left(X_{\text {uni }}, K(\mathscr{E}) \otimes K\left(\mathscr{E}^{\prime}\right)\right) \simeq \mathbf{H}_{c}^{0}\left(\mathscr{O}, K(\mathscr{E}) \otimes K\left(\mathscr{E}^{\prime}\right)\right)
$$

Since the cohomology in the right hand side coincides with $H_{c}^{2 \operatorname{dim} \mathscr{O}}\left(\mathscr{O}, \mathscr{E} \otimes \mathscr{E}^{\prime}\right)$, we obtain the required formula. The lemma is proved.

5.15. We are now ready to prove (i) of Theorem 5.2. Put $K=\pi_{*} K_{\overline{\mathscr{E}}_{1}}, K_{1}=$ $\left.K[-r]\right|_{X_{\text {uni }}}=\left(\pi_{1}\right)_{*} K \frac{1}{\mathscr{E}_{1}}$. We have End $K \simeq \mathscr{A}_{\mathscr{E}_{1}}$. Let

$$
\alpha: \text { End } K \rightarrow \text { End } K_{1}
$$

be the natural homomorphism. We show that $\alpha$ gives rise to an isomorphism. Let us consider the sheaf $\widetilde{\mathscr{T}}$ defined in $(4.3 .2)$ with $\mathscr{E}_{1}^{\prime}=\mathscr{E}_{1}^{*}$. Note that $K_{\overline{\mathscr{E}}_{1}^{*}}=D\left(K_{\overline{\mathscr{E}}_{1}}\right)$ and $\pi_{*} K_{\overline{\mathscr{E}}_{1}^{*}}=D(K)$, where $D$ is the Verdier dual operator. Using the expression (4.11.1), we have

$$
\begin{aligned}
\widetilde{\mathscr{T}_{1}} & \simeq \mathbf{H}_{c}^{-2 r}\left(Z^{\prime}, K_{\overline{\mathscr{E}}_{1}} \otimes D\left(K_{\overline{\mathscr{E}}_{1}}\right)\right) \\
& \simeq \mathbf{H}_{c}^{-2 r}\left(X_{\text {uni }},\left.\left.K\right|_{X_{\text {uni }}} \otimes D(K)\right|_{X_{\text {uni }}}\right) \\
& =\mathbf{H}_{c}^{0}\left(X_{\text {uni }}, K_{1} \otimes D\left(K_{1}\right)\right) .
\end{aligned}
$$


Let $n_{\mathscr{E}}$ be the multiplicity of $K(\mathscr{E})$ appearing in the decomposition of the semisimple perverse sheaf $K_{1}$. Since $n_{\mathscr{E}}$ coincides with the multiplicity of $K\left(\mathscr{E}^{*}\right)=D(K(\mathscr{E}))$ in the decomposition of $D K_{1}$, by using Lemma 5.14, we have

$$
\operatorname{dim} \widetilde{\mathscr{T}_{1}}=\operatorname{dim} \mathbf{H}_{c}^{0}\left(X_{\text {uni }}, K_{1} \otimes D\left(K_{1}\right)\right)=\sum_{(\mathscr{O}, \mathscr{E}) \in \mathscr{N}_{G}} n_{\mathscr{E}}^{2}
$$

On the other hand, by Proposition $4.12, \operatorname{dim} \widetilde{\mathscr{T}}_{1}=\operatorname{dim} \mathscr{A}_{\mathscr{E}_{1}}=\operatorname{dim}$ End $K$. Since $\operatorname{dim}$ End $K_{1}=\sum n_{\mathscr{E}}^{2}$, we have $\operatorname{dim}$ End $K=\operatorname{dim}$ End $K_{1}$. Hence it is enough to show that $\alpha$ is injective. Now $K_{1}$ can be decomposed as $K_{1} \simeq \bigoplus_{\rho \in \mathscr{A}_{\mathscr{E}_{1}}} \rho \otimes\left(\left.K_{\rho}\right|_{X_{\text {uni }}}\right)$, up to shift, and the $\mathscr{A}_{\mathscr{E}_{1}} \otimes \mathscr{A}_{\mathscr{E}_{1}}^{0}$-module structure of $\widetilde{\mathscr{T}_{1}}=\mathbf{H}_{c}^{0}\left(X_{\text {uni }}, K_{1} \otimes D\left(K_{1}\right)\right)$ is determined from this decomposition. By Proposition 4.12 , we know that $\widetilde{\mathscr{T}_{1}}$ is the two-sided regular representation of $\mathscr{A}_{\mathscr{E}_{1}}$. In particular, $\left.K_{\rho}\right|_{X_{\text {uni }}} \neq 0$ for any $\rho \in \mathscr{A}_{\mathscr{E}_{1}}^{\wedge}$. Since $\left.K_{\rho}\right|_{X_{\text {uni }}}$ is a direct summand of $K_{1},\left.K_{\rho}\right|_{X_{\text {uni }}}$ is a sum of various $K(\mathscr{E})$, at least one summand. Thus $\alpha$ is injective, and so $\alpha$ gives an isomorphism. (5.2.1) follows from this. From the above discussion, we have $\left.K_{\rho}\right|_{X_{\text {uni }}} \simeq \operatorname{IC}(\overline{\mathscr{O}}, \mathscr{E})[\operatorname{dim} \mathscr{O}+r]$, which is equivalent to (5.2.2). Thus (i) of Theorem 5.2 is proved.

5.16. We prove (ii) and (iii) of Theorem 5.2. Assume that $\mathscr{O} \subset X$. First we show that the homomorphism in (iii) is surjective. For this, it is enough to see that $\mathbf{H}_{c}^{2 d_{\mathscr{O}}-\operatorname{dim} X}\left(\pi^{-1}(x)-\pi^{-1}(x)_{\beta_{0}}, K_{\overline{\mathscr{E}}_{1}}\right)=0$ for any $x \in \mathscr{O}$, thus enough to see that $\mathbf{H}_{c}^{2 d_{\mathscr{O}}-\operatorname{dim} X}\left(\pi^{-1}(x)_{\beta}, K_{\mathscr{\mathscr { E }}_{1}}\right)=0$ for any $\beta \neq \beta_{0}$. (Here we put $\pi^{-1}(x)_{\beta}=\pi^{-1}(x) \cap \widetilde{X}_{\beta}$ under the notation in 4.3.) By using the hypercohomology spectral sequence, we have only to show that if $H_{c}^{i}\left(\pi^{-1}(x)_{\beta}, \mathscr{H}^{j} K_{\overline{\mathscr{E}}_{1}}\right) \neq 0$, then $i+j<2 d_{\mathscr{O}}-\operatorname{dim} X$. If the cohomology is non-zero, Proposition 2.2 (ii) implies that

$$
i \leq 2 \operatorname{dim} \pi^{-1}(x)_{\beta} \leq 2 d_{\mathscr{O}}-\left(\operatorname{dim} \mathscr{O}_{L}-\operatorname{dim} \mathscr{O}_{\beta}\right),
$$

and $j<-\operatorname{dim} \tilde{X}_{\beta}=-\operatorname{dim} X+\left(\operatorname{dim} \mathscr{O}_{L}-\operatorname{dim} \mathscr{O}_{\beta}\right)$ since $\beta \neq \beta_{0}$. Thus $i+j<$ $2 d_{\mathscr{O}}-\operatorname{dim} X$ as asserted. Hence the homomorphism is surjective.

For an $H$-equivariant simple local system $\mathscr{E}$ on $\mathscr{O}$, we denote by $m_{\mathscr{E}}$ the multiplicity of $\mathscr{E}$ in $\left.R^{2 d_{\mathscr{O}}} f_{!} \overline{\mathscr{E}}_{1}\right|_{\mathscr{O}}$, and by $\widetilde{m}_{\mathscr{E}}$ the multiplicity of $\mathscr{E}$ in $\left.\mathscr{H}^{2 d_{\mathscr{O}}-\operatorname{dim} X} K\right|_{\mathscr{O}}$. Since the homomorphism in (iii) is surjective, we have

$$
m_{\mathscr{E}} \geq \widetilde{m}_{\mathscr{E}}
$$

By $(5.2 .2)$, we have $\left.\left.\mathscr{H}^{2 d_{\mathscr{O}}}\left(K_{\rho}[-\operatorname{dim} X]\right)\right|_{\mathscr{O}} \simeq \mathscr{H}^{0}(\mathrm{IC}(\overline{\mathscr{O}}, \mathscr{E}))\right|_{\mathscr{O}} \simeq \mathscr{E}$, and so $\widetilde{m}_{\mathscr{E}}=$ $n_{\mathscr{E}}$. Hence by the discussion in 5.15 , we have

$$
\sum_{(\mathscr{O}, \mathscr{E}) \in \mathscr{N}_{G}} \widetilde{m}_{\mathscr{E}}^{2}=\operatorname{dim} \mathscr{A}_{\mathscr{E}_{1}} .
$$


We shall prove that

$$
\sum_{(\mathscr{O}, \mathscr{E}) \in \mathscr{N}_{G}} m_{\mathscr{E}}^{2}=\operatorname{dim} \mathscr{A}_{\mathscr{E}}
$$

From the definition of $m_{\mathscr{E}}$, we have

$$
\operatorname{dim} H_{c}^{2 \operatorname{dim} \mathscr{O}}\left(\mathscr{O}, R^{2 d_{\mathscr{O}}} f_{!}\left(\overline{\mathscr{E}}_{1}\right) \otimes R^{2 d_{\mathscr{O}}} f_{!}\left(\overline{\mathscr{E}}_{1}^{*}\right)\right)=\sum_{\mathscr{E}} m_{\mathscr{E}}^{2}
$$

where in the sum, $\mathscr{E}$ runs over all the $H$-equivariant local systems on a fixed $\mathscr{O}$. Let $Z_{0}=\widetilde{X}_{0} \times_{X} \widetilde{X}_{0}$ and $p_{1}: Z_{0} \rightarrow X$ be the first projection. For an $H$-orbit $\mathscr{O} \subset X_{\text {uni }}$, put $Z_{0, \mathscr{O}}=p_{1}^{-1}(\mathscr{O})$. We have a natural map $Z_{0, \mathscr{O}} \rightarrow \mathscr{O}$, and all fibres have dimension $\leq 2 d_{\mathscr{O}}$. Since $2 d_{\mathscr{O}}+\operatorname{dim} \mathscr{O}=d_{0}$, where $d_{0}$ is as in $(4.3 .1)$, by using the Leray spectral sequence, we have

$$
H_{c}^{2 d_{0}}\left(Z_{0, \mathscr{O}}, \overline{\mathscr{E}}_{1} \otimes \overline{\mathscr{E}}_{1}^{*}\right) \simeq H_{c}^{2 \operatorname{dim} \mathscr{O}}\left(\mathscr{O}, R^{2 d_{\mathscr{O}}} f_{!}\left(\overline{\mathscr{E}}_{1}\right) \otimes R^{2 d_{\mathscr{O}}} f_{!}\left(\overline{\mathscr{E}}_{1}^{*}\right)\right)
$$

Now $Z_{\beta_{0}, \beta_{0}}^{1}=\coprod_{\mathscr{O}} Z_{0, \mathscr{O}}$ gives a partition of $Z_{\beta_{0}, \beta_{0}}^{1}=p_{1}^{-1}\left(X_{\text {uni }}\right)$ by locally closed pieces $Z_{0, \mathscr{O}}$, where $\operatorname{dim} Z_{0, \mathscr{O}} \leq d_{0}$. Hence

$$
\begin{aligned}
\sum_{(\mathscr{O}, \mathscr{E})} m_{\mathscr{E}}^{2} & =\sum_{\mathscr{O}} \operatorname{dim} H_{c}^{2 d_{0}}\left(Z_{0, \mathscr{O}}, \overline{\mathscr{E}}_{1} \otimes \overline{\mathscr{E}}_{1}^{*}\right) \\
& =\operatorname{dim} H_{c}^{2 d_{0}}\left(Z_{\beta_{0}, \beta_{0}}^{1}, \overline{\mathscr{E}}_{1} \otimes \overline{\mathscr{E}}_{1}^{*}\right) \\
& =\operatorname{dim} \mathscr{T}_{1}=\operatorname{dim} \mathscr{A}_{\mathscr{E}_{1}} .
\end{aligned}
$$

Hence (5.16.3) holds. Comparing (5.16.2) and (5.16.3), we see, by (5.16.1), that $m_{\mathscr{E}}=\widetilde{m}_{\mathscr{E}}$. This shows that the natural homomorphism in (iii) is an isomorphism.

By Lemma 5.8, the condition $(\mathscr{O}, \mathscr{E}) \in \mathscr{N}_{G}$ belongs to $\xi=\left(L \subset P, \mathscr{O}_{L}, \mathscr{E}_{1}^{\dagger}\right) \in$ $\mathscr{S}_{G}$, i.e., $(\mathscr{O}, \mathscr{E}) \in \mathscr{N}_{G}^{(\xi)}$, is equivalent to the condition that $m_{\mathscr{E}} \neq 0$. On the other hand, let $\widetilde{\mathscr{N}}_{G}^{(\xi)}$ be the set of $(\mathscr{O}, \mathscr{E}) \in \mathscr{N}_{G}$ such that $\operatorname{IC}(\overline{\mathscr{O}}, \mathscr{E})[\operatorname{dim} \mathscr{O}]$ appears as a direct summand of $\pi_{*} K_{\overline{\mathscr{E}}_{1}}$ for $\xi=\left(L \subset P, \mathscr{O}_{L}, \mathscr{E}_{1}^{\dagger}\right)$. Then by Theorem 5.2 (i), the condition $(\mathscr{O}, \mathscr{E}) \in \widetilde{\mathscr{N}}_{G}^{(\xi)}$ is equivalent to the condition that $\widetilde{m}_{\mathscr{E}} \neq 0$. Since $m_{\mathscr{E}}=\widetilde{m}_{\mathscr{E}}$, we conclude that $\mathscr{N}_{G}^{(\xi)}=\widetilde{\mathscr{N}}_{G}^{(\xi)}$. Now (ii) and (iii) follows from (5.7.1) and Lemma 5.8. This completes the proof of the theorem.

Remark 5.17. It is likely that the previous discussion for establishing the generalized Springer correspondence will work for a symmetric space of general type if it satisfies the condition that $\Delta_{0}^{+}=\emptyset$.

\section{Restriction Theorem}

6.1. Let $P=L U_{P}$ be as in 3.1. Hence $L^{\theta} \simeq\left(G L_{1}\right)^{a} \times G L_{N_{0}}^{\theta}$ with $N_{0}=N-2 a$. We consider a $\theta$-stable parabolic subgroup $Q$ of $G$ containing $P$, and the $\theta$-stable Levi 
subgroup $M$ of $Q$ containing $L$. Here we assume that $M^{\theta} \simeq\left(G L_{1}\right)^{a^{\prime}} \times G L_{N_{0}^{\prime}}^{\theta}$, where $N_{0}^{\prime}=N-2 a^{\prime}$ with $a \geq a^{\prime}$. Let $\mathscr{O}_{L}$ be an $L_{H^{-}}$orbit in $L_{\text {uni }}^{\iota \theta}$, and $\mathscr{E}_{1}^{\dagger}$ a cuspidal local system on $\mathscr{O}_{L}$. Put $\Sigma=Z_{L}^{\iota \theta} \mathscr{O}_{L}$, and $\mathscr{E}_{1}=\overline{\mathbf{Q}}_{l} \otimes \mathscr{E}_{1}^{\dagger}$. We consider the complex $\pi_{*} K_{\overline{\mathscr{E}}_{1}}$ on $X$ obtained from the triple $\left(L \subset P, \mathscr{O}_{L}, \mathscr{E}_{1}^{\dagger}\right) \in \mathscr{S}_{G}$. Then the endomorphism algebra $\operatorname{End}\left(\pi_{*} K_{\overline{\mathscr{E}}_{1}}\right)$ is isomorphic to $\mathscr{A}_{\mathscr{E}_{1}}$, which is a twisted group algebra of $\mathscr{W}_{\mathscr{E}_{1}} \subset$ $\mathscr{W}_{1} \simeq S_{a}$. By applying a similar discussion for $\left(L \subset M \cap P, \mathscr{O}_{L}, \mathscr{E}_{1}^{\dagger}\right) \in \mathscr{S}_{M}$, one can obtain a complex $\pi_{*}^{\prime} K_{\mathscr{E}_{1}}^{\prime}$ on $X^{\prime}$. Then the endomorphism algebra $\operatorname{End}\left(\pi_{*}^{\prime} K_{\mathscr{E}_{1}}^{\prime}\right) \simeq \mathscr{A}_{\mathscr{E} 1}^{\prime}$, which is isomoprhic to a twisted group algebra of $\mathscr{W}_{\mathscr{E}_{1}}^{\prime} \subset \mathscr{W}_{1}^{\prime} \simeq S_{a^{\prime}}$. (Here we denote by $X^{\prime}, \pi^{\prime}$, etc. by attaching the primes to express the objects for $M$ corresponding to $X, \pi$, etc. for $G) . \mathscr{A}_{\mathscr{E}_{1}}^{\prime}$ is canonically identified with the subalgebra of $\mathscr{A}_{\mathscr{E}_{1}}$.

We now apply the generalized Springer correspondence for $H$ and $M_{H}=\left(M^{\theta}\right)^{0}$. By Theorem 5.2 (ii), if $\left(\mathscr{O}^{\prime}, \mathscr{E}^{\prime}\right) \in \mathscr{N}_{M}$ belongs to the series $\left(L \subset M \cap P, \mathscr{O}_{L}, \mathscr{E}_{1}^{\dagger}\right) \in$ $\mathscr{S}_{M}$, then an irreducible representation $\rho^{\prime} \in\left(\mathscr{A}_{\mathscr{E} 1}^{\prime}\right)^{\wedge}$ is determined from $\left(\mathscr{O}^{\prime}, \mathscr{E}^{\prime}\right)$. On the other hand, if $(\mathscr{O}, \mathscr{E}) \in \mathscr{N}_{G}$ belongs to the series $\left(L \subset P, \mathscr{O}_{L}, \mathscr{E}_{1}^{\dagger}\right) \in \mathscr{S}_{G}$, then an irreducible representation $\rho \in \mathscr{A}_{\mathscr{E}_{1}}^{\wedge}$ is determined. We will describe the relationship between $\rho^{\prime}$ and $\rho$.

Take $\mathscr{O}$ and $\mathscr{O}^{\prime}$ as above. Let $f_{\mathscr{O}, \mathscr{O}^{\prime}}: \eta_{Q}^{-1}\left(\mathscr{O}^{\prime}\right) \cap \mathscr{O} \rightarrow \mathscr{O}^{\prime}$ be the restriction of the map $\eta_{Q}: Q^{\iota \theta} \rightarrow M^{\iota \theta}$. We define an integer $m_{\mathscr{E}, \mathscr{E}^{\prime}}$ as the multiplicity of $\mathscr{E}^{\prime}$ in the

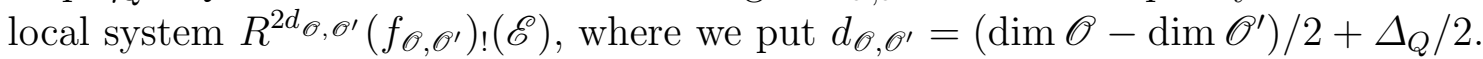
The following result is an analogue of Lusztig's restriction theorem [L1, Theorem 8.3].

Theorem 6.2. Assume that $\left(\mathscr{O}^{\prime}, \mathscr{E}^{\prime}\right) \in \mathscr{N}_{M}$ belongs to $\left(L \subset M \cap P, \mathscr{O}_{L}, \mathscr{E}_{1}^{\dagger}\right) \in \mathscr{S}_{M}$.

(i) If $m_{\mathscr{E}, \mathscr{E}^{\prime}} \neq 0$, then $(\mathscr{O}, \mathscr{E}) \in \mathscr{N}_{G}$ belongs to $\left(L \subset P, \mathscr{O}_{L}, \mathscr{E}_{1}^{\dagger}\right)$.

(ii) Assume that $(\mathscr{O}, \mathscr{E}) \in \mathscr{N}_{G}$ belongs to $\left(L \subset P, \mathscr{O}_{L}, \mathscr{E}_{1}^{\dagger}\right)$, and let $\rho \in \mathscr{A}_{\mathscr{E}_{1}}^{\wedge}$ (resp. $\left.\rho^{\prime} \in\left(\mathscr{A}_{\mathscr{E}_{1}}^{\prime}\right)^{\wedge}\right)$ be the irreducible representation corresponding to $(\mathscr{O}, \mathscr{E})$ (resp. $\left.\left(\mathscr{O}^{\prime}, \mathscr{E}^{\prime}\right)\right)$. Then we have

$$
\left\langle\left.\rho\right|_{\mathscr{A}_{\mathscr{E}_{1}}^{\prime}}, \rho^{\prime}\right\rangle_{\mathscr{A}_{\mathscr{E}_{1}}^{\prime}}=m_{\mathscr{E}, \mathscr{E}^{\prime}}
$$

where the left hand side means the multiplicity of $\rho^{\prime}$ in the restriction of $\rho$ on $\mathscr{A}_{\mathscr{E} 1}^{\prime}$.

6.3. The remainder of this section is devoted to the proof of the theorem. The proof is done by a similar strategy as in [L1]. Take $\left(L \subset P, \mathscr{O}_{L}, \mathscr{E}_{1}^{\dagger}\right) \in \mathscr{S}_{G}$, and let $Y=\bigcup_{g \in H} g\left(\Sigma_{\text {reg }} \mathscr{D}_{P}^{0}\right) g^{-1}, \bar{Y}=X=\bigcup_{g \in H} g \eta_{P}^{-1}(\bar{\Sigma}) g^{-1}$ be as in Section 3. By replacing $G, P$ by $M, M \cap P$, we obtain similar varieties,

$$
Y^{M}=\bigcup_{g_{1} \in M_{H}} g_{1}\left(\Sigma_{\text {reg }} \mathscr{D}_{M \cap P}^{0}\right) g_{1}^{-1}, \quad \overline{Y^{M}}=X^{M}=\bigcup_{g_{1} \in M_{H}} g_{1} \eta_{M \cap P}^{-1}(\bar{\Sigma}) g_{1}^{-1} .
$$

Consider the following varieties, 


$$
\begin{aligned}
\widetilde{Y} & =\left\{\left(x, g L_{H}\right) \in G^{\iota \theta} \times H / L_{H} \mid g^{-1} x g \in \Sigma_{\mathrm{reg}} \mathscr{D}_{P}^{0}\right\}, \\
\widetilde{Y}_{M} & =\left\{\left(x, g M_{H}\right) \in G^{\iota \theta} \times H / M_{H} \mid g^{-1} x g \in Y^{M} \mathscr{D}_{Q}^{0}\right\}, \\
\widetilde{X} & =\left\{\left(x, g P_{H}\right) \in G^{\iota \theta} \times H / P_{H} \mid g^{-1} x g \in \eta_{P}^{-1}(\bar{\Sigma})\right\}, \\
\widetilde{X}_{Q} & =\left\{\left(x, g Q_{H}\right) \in G^{\iota \theta} \times H / Q_{H} \mid g^{-1} x g \in \eta_{Q}^{-1}\left(X^{M}\right)\right\} .
\end{aligned}
$$

Note that $Y^{M} \mathscr{D}_{Q}^{0} \subset Q^{\iota \theta}$ since

$$
g_{1}\left(\Sigma_{\text {reg }} \mathscr{D}_{M \cap P}^{0}\right) g_{1}^{-1} \cdot \mathscr{D}_{Q}^{0}=g_{1}\left(\Sigma_{\text {reg }} \mathscr{D}_{M \cap P}^{0} \mathscr{D}_{Q}^{0}\right) g_{1}^{-1}=g_{1}\left(\Sigma_{\text {reg }} \mathscr{D}_{P}^{0}\right) g_{1}^{-1} \subset Q^{\iota \theta}
$$

for $g_{1} \in M_{H}$. Let $\pi_{0}: \widetilde{Y} \rightarrow Y, \pi: \widetilde{X} \rightarrow X$ be the first projections as before. We have the following commutative diagram,

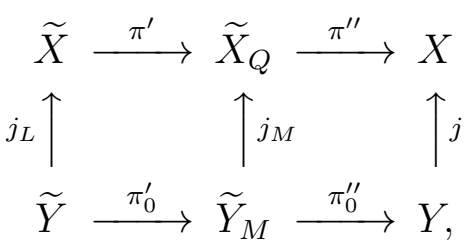

where $\pi^{\prime}:\left(x, g P_{H}\right) \mapsto\left(x, g Q_{H}\right), \pi^{\prime \prime}:\left(x, g Q_{H}\right) \mapsto x, \pi_{0}^{\prime}:\left(x, g L_{H}\right) \mapsto\left(x, g M_{H}\right), \pi_{0}^{\prime \prime}:$ $\left(x, g M_{H}\right) \mapsto x$. (Note that $\pi_{0}^{\prime}$ is well-defined since $g_{1}^{-1} x g_{1} \in Y^{M} \mathscr{D}_{Q}^{0}$ for $x \in \Sigma_{\text {reg }} \mathscr{D}_{P}^{0}$, $g_{1} \in M_{H}$ by $\left.\mathscr{D}_{P}^{0}=\mathscr{D}_{P \cap M}^{0} \times \mathscr{D}_{Q}^{0}.\right) \quad$ Moreover, $j_{L}:\left(x, g L_{H}\right) \mapsto\left(x, g P_{H}\right), j_{M}$ : $\left(x, g M_{H}\right) \mapsto\left(x, g Q_{H}\right), j: x \mapsto x$ are natural inclusions. By definition, $\pi=$ $\pi^{\prime \prime} \circ \pi^{\prime}, \pi_{0}=\pi_{0}^{\prime \prime} \circ \pi_{0}^{\prime}$. By Lemma 3.5, $j_{L}, j$ give embeddings of $\tilde{Y}, Y$ into $\tilde{X}, X$ as open dense smooth subsets. Since $\widetilde{Y}_{M}=\left(\pi^{\prime \prime}\right)^{-1}(Y), \widetilde{Y}_{M}$ is open dense in $\widetilde{X}_{Q}$. Here $\widetilde{Y}_{M} \simeq H \times{ }^{M_{H}}\left(Y^{M} \mathscr{D}_{Q}^{0}\right)$. Since $Y^{M}$ is smooth by Lemma 3.5 , and so $Y^{M} \mathscr{D}_{Q}^{0}$ is smooth, thus $\widetilde{Y}_{M}$ is smooth.

Recall that $\pi_{0}: \widetilde{Y} \rightarrow Y$ is a finite Galois covering with Galois group $\mathscr{W}_{1} \simeq S_{a}$. $\mathscr{L}=\left(\pi_{0}\right)_{*} \widetilde{\mathscr{E}}_{1}$ is a semisimple local system on $Y$ and we have End $\mathscr{L} \simeq \mathscr{A}_{\mathscr{E}_{1}}$. Thus $\mathscr{L}$ is decomposed as

$$
\mathscr{L} \simeq \bigoplus_{\rho \in \mathscr{A}_{\hat{\mathscr{E}}_{1}}} \rho \otimes \mathscr{L}_{\rho}
$$

where $\mathscr{L}_{\rho}=\operatorname{Hom}_{\mathscr{A}_{\mathscr{E}_{1}}}(\rho, \mathscr{L})$. Similarly, $\pi_{0}^{\prime}: \widetilde{Y} \rightarrow \widetilde{Y}_{M}$ is a finite Galois covering with group $\mathscr{W}_{1}^{\prime} \simeq S_{a^{\prime}}$. Thus $\mathscr{L}^{\prime}=\left(\pi_{0}^{\prime}\right)_{*} \widetilde{\mathscr{E}}_{1}$ is a semisimple local system on $\widetilde{Y}_{M}$, and we have End $\mathscr{L}^{\prime} \simeq \mathscr{A}_{\mathscr{E}_{1}}^{\prime}$. We have a decomposition into simple local systems,

$$
\mathscr{L}^{\prime} \simeq \bigoplus_{\rho^{\prime} \in\left(\mathscr{A}_{\mathscr{E}_{1}}^{\prime}\right)^{\wedge}} \rho^{\prime} \otimes \mathscr{L}_{\rho^{\prime}}^{\prime}
$$


where $\mathscr{L}_{\rho^{\prime}}^{\prime}=\operatorname{Hom}_{\mathscr{A}_{\mathscr{E}_{1}}^{\prime}}\left(\rho^{\prime}, \mathscr{L}^{\prime}\right)$. Since $\mathscr{L} \simeq\left(\pi_{0}^{\prime \prime}\right)_{*}\left(\pi_{0}^{\prime}\right)_{*} \widetilde{\mathscr{E}}_{1} \simeq\left(\pi_{0}^{\prime \prime}\right)_{*} \mathscr{L}^{\prime}$, by applying $\left(\pi_{0}^{\prime \prime}\right)_{*}$ on both sides of $(6.3 .3)$, we have

$$
\mathscr{L} \simeq \bigoplus_{\rho^{\prime} \in\left(\mathscr{A}_{\mathscr{E}_{1}}^{\prime}\right)^{\wedge}} \rho^{\prime} \otimes\left(\pi_{0}^{\prime \prime}\right)_{*} \mathscr{L}_{\rho^{\prime}}^{\prime}
$$

By comparing (6.3.2) and (6.3.4), we see that $\left(\pi_{0}^{\prime \prime}\right)_{*} \mathscr{L}_{\rho^{\prime}}^{\prime}$ is a semisimple local system whose simple components are of the form $\mathscr{L}_{\rho}$ for some $\rho \in \mathscr{A}_{\mathscr{E}_{1}}^{\wedge}$. Since the action of $\mathscr{A}_{\mathscr{E} 1}^{\prime}$ on $\mathscr{L}$ in (6.3.4) is the restriction to $\mathscr{A}_{\mathscr{E}_{1}}^{\prime}$ of the action of $\mathscr{A}_{\mathscr{E}_{1}}$ on $\mathscr{L}$ in (6.3.2), we obtain the following decomposition into simple local systems.

$$
\left(\pi_{0}^{\prime \prime}\right)_{*} \mathscr{L}_{\rho^{\prime}}^{\prime} \simeq \bigoplus_{\rho \in \mathscr{A}_{\hat{\mathscr{E}}_{1}}} \mathscr{L}_{\rho} \otimes \overline{\mathbf{Q}}_{l}^{\left\langle\rho, \rho^{\prime}\right\rangle}
$$

where $\left\langle\rho, \rho^{\prime}\right\rangle=\left\langle\left.\rho\right|_{\mathscr{A}_{\mathscr{E}_{1}}^{\prime}}, \rho^{\prime}\right\rangle_{\mathscr{A}_{\mathscr{E}_{1}}^{\prime}}$ is the multiplicity of $\rho^{\prime}$ in the restriction of $\rho$ on $\mathscr{A}_{\mathscr{E}_{1}}^{\prime}$.

6.4. We now consider $K_{\overline{\mathscr{E}}_{1}}=\operatorname{IC}\left(\widetilde{X}, \overline{\mathscr{E}}_{1}\right)[\operatorname{dim} \tilde{X}]$. By Proposition $3.8, \pi_{*} K_{\overline{\mathscr{E}}_{1}}$ is a semisimple perverse sheaf on $X$, and is decomposed into simple perverse sheaves

$$
\pi_{*} K_{\overline{\mathscr{E}}_{1}} \simeq \bigoplus_{\rho \in \mathscr{A}_{\mathscr{E}_{1}}} \rho \otimes A_{\rho}
$$

where $A_{\rho}=\operatorname{IC}\left(X, \mathscr{L}_{\rho}\right)[\operatorname{dim} X]$.

Next we shall show that

$$
\pi_{*}^{\prime} K_{\overline{\mathscr{E}}_{1}} \simeq \operatorname{IC}\left(\widetilde{X}_{Q}, \mathscr{L}^{\prime}\right)\left[\operatorname{dim} \tilde{X}_{Q}\right]
$$

Under the isomorphisms

$$
\begin{aligned}
H \times{ }^{P_{H}}\left(\eta_{P}^{-1}(\bar{\Sigma})\right) & \stackrel{\sim}{\rightarrow} \widetilde{X} \\
H \times{ }^{Q_{H}}\left(\eta_{Q}^{-1}\left(X^{M}\right)\right) & \stackrel{\sim}{\rightarrow} \widetilde{X}_{Q}
\end{aligned}
$$

consider the following commutative diagram

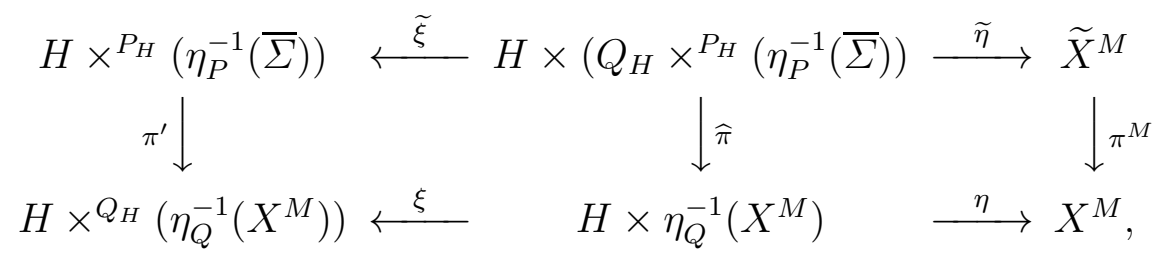


where $\xi$ is the quotient map under the free action of $Q_{H}$ on $H \times \eta_{Q}^{-1}\left(X^{M}\right)$ by $q:(g, y) \mapsto\left(g q^{-1}, q y q^{-1}\right), \widetilde{\xi}$ is the quotient map under the free action of $Q_{H}$ on $H \times\left(Q_{H} \times{ }^{P_{H}}\left(\eta_{P}^{-1}(\bar{\Sigma})\right)\right.$ as above, under the natural isomorphism

$$
H \times{ }^{Q_{H}}\left(Q_{H} \times^{P_{H}}\left(\eta_{P}^{-1}(\bar{\Sigma})\right)\right) \simeq H \times{ }^{P_{H}}\left(\eta_{P}^{-1}(\bar{\Sigma})\right) .
$$

$\eta$ is the composite of the projection $H \times \eta_{Q}^{-1}\left(X^{M}\right) \rightarrow \eta_{Q}^{-1}\left(X^{M}\right)$ and the map $\eta_{Q}$. The map $\pi^{M}: \widetilde{X}^{M} \rightarrow X^{M}$ is the map analogous to $\pi: \widetilde{X} \rightarrow X$ obtained by replacing $G$ by $M . \widetilde{\eta}$ is the composite of the projection $H \times\left(Q_{H} \times{ }^{P_{H}}\left(\eta_{P}^{-1}(\bar{\Sigma})\right) \rightarrow\right.$ $Q_{H} \times{ }^{P_{H}}\left(\eta_{P}^{-1}(\bar{\Sigma})\right)$ and the natural map

$$
Q_{H} \times{ }^{P_{H}}\left(\eta_{P}^{-1}(\bar{\Sigma})\right) \rightarrow M_{H} \times^{\left(P_{H} \cap M_{H}\right)}\left(\eta_{P \cap M}^{-1}(\bar{\Sigma})\right) \simeq \widetilde{X}^{M}
$$

obtained from the projection $Q \rightarrow M$. Under the isomorphisms in (6.4.3), $\pi^{\prime}$ coincides with the map induced from the natural inclusion $H \times \eta_{P}^{-1}(\bar{\Sigma}) \hookrightarrow H \times \eta_{Q}^{-1}\left(X^{M}\right)$. $\widehat{\pi}=\mathrm{id} \times \widehat{\pi}_{1}$, where $\widehat{\pi}_{1}: Q_{H} \times{ }^{P_{H}}\left(\eta_{P}^{-1}(\bar{\Sigma})\right) \rightarrow \eta_{Q}^{-1}\left(X^{M}\right)$ induced from $(g, x) \mapsto g x g^{-1}$.

Here $\eta, \widetilde{\eta}$ are $Q_{H^{-}}$equivariant with respect to the natural action of $M_{H}$ (and the trivial action of $U_{Q_{H}}$ ) on $\tilde{X}^{M}$ and on $X^{M}$. Note that two squares are cartesian squares.

By applying the construction of $K_{\overline{\mathscr{E}}_{1}}=\operatorname{IC}\left(\widetilde{X}, \overline{\mathscr{E}}_{1}\right)[\operatorname{dim} \tilde{X}]$ to $M$, one can define the perverse sheaf $K_{\overline{\mathscr{E}}_{1}^{M}}=\operatorname{IC}\left(\widetilde{X}^{M}, \overline{\mathscr{E}}_{1}^{M}\right)\left[\operatorname{dim} \widetilde{X}^{M}\right]$ on $\widetilde{X}^{M}$, and we obtain an $M_{H^{-}}$equivariant semisimple perverse sheaf $\left(\pi^{M}\right)_{*} K_{\overline{\mathscr{E}}_{1}^{M}}$ on $X^{M}$. Here $\eta$ is a smooth morphism with connected fibre isomorphic to $H \times U_{Q}^{\iota \theta}$, and is $Q_{H}$-equivariant. Then $\eta^{*}\left(\pi^{M}\right)_{*} K_{\overline{\mathscr{E}}_{1} M}[\alpha]$ is a $Q_{H^{-}}$equivariant perverse sheaf on $H \times \eta_{Q}^{-1}\left(X^{M}\right)$, where $\alpha=\operatorname{dim} H+\operatorname{dim} U_{Q}^{\iota \theta}$. On the other hand, since $\xi$ is a $Q_{H}$-principal bundle, there exists a unique perverse sheaf $K_{1}$ on $H \times{ }^{Q_{H}}\left(\eta_{Q}^{-1}\left(X^{M}\right)\right)$ such that

$$
\xi^{*} K_{1}[\beta] \simeq \eta^{*}\left(\pi^{M}\right)_{*} K_{\mathscr{\mathscr { E }}_{1}^{M}}[\alpha]
$$

where $\beta=\operatorname{dim} Q_{H}$.

A similar construction works also for the $M_{H}$-equivariant perverse sheaf $K_{\overline{\mathscr{E}}_{1}^{M}}$ on $\widetilde{X}^{M}$, and one can find a perverse sheaf $K_{2}$ on $H \times^{P_{H}}\left(\eta_{P}^{-1}(\bar{\Sigma})\right)$, unique up to isomorphism, such that

$$
\widetilde{\xi}^{*} K_{2}[\beta] \simeq \widetilde{\eta}^{*} K_{\mathscr{E}_{1}^{M}}[\alpha]
$$

Here, if we note that the two squares in the diagram (6.4.4) are cartesian squares, by using the proper base change theorem, (6.4.5) and (6.4.6) imply that

$$
K_{1} \simeq \pi_{*}^{\prime} K_{2} .
$$


Thus, in order to prove (6.4.2), it is enough to see that

$$
K_{2} \simeq \operatorname{IC}\left(\widetilde{X}, \overline{\mathscr{E}}_{1}\right)[\operatorname{dim} \tilde{X}], \quad K_{1} \simeq \operatorname{IC}\left(\widetilde{X}_{Q},\left(\pi_{0}^{\prime}\right)_{*} \widetilde{\mathscr{E}}_{1}\right)\left[\operatorname{dim} \widetilde{X}_{Q}\right]
$$

It follows from the construction that $K_{1}$ (resp. $K_{2}$ ) is an intersection cohomology complex on $\tilde{X}_{Q}$ (resp. $\widetilde{X}$ ) whose support is $\widetilde{X}_{Q}$ (resp. $\widetilde{X}$ ). Hence to prove $(6.4 .8)$, we have only to show that

$$
\left.K_{2}\right|_{\widetilde{Y}} \simeq \widetilde{\mathscr{E}}_{1}[\operatorname{dim} \tilde{Y}],\left.\quad K_{1}\right|_{\widetilde{Y}_{M}} \simeq\left(\pi_{0}^{\prime}\right)_{*} \widetilde{\mathscr{E}}_{1}\left[\operatorname{dim} \widetilde{Y}_{M}\right]
$$

Under the isomorphisms

$$
\begin{aligned}
\tilde{Y} & \simeq H \times{ }^{L_{H}}\left(\Sigma_{\mathrm{reg}} \mathscr{D}_{P}^{0}\right), \\
\widetilde{Y}_{M} & \simeq H \times \times^{M_{H}}\left(Y^{M} \mathscr{D}_{Q}^{0}\right),
\end{aligned}
$$

we have the following commutative diagram.

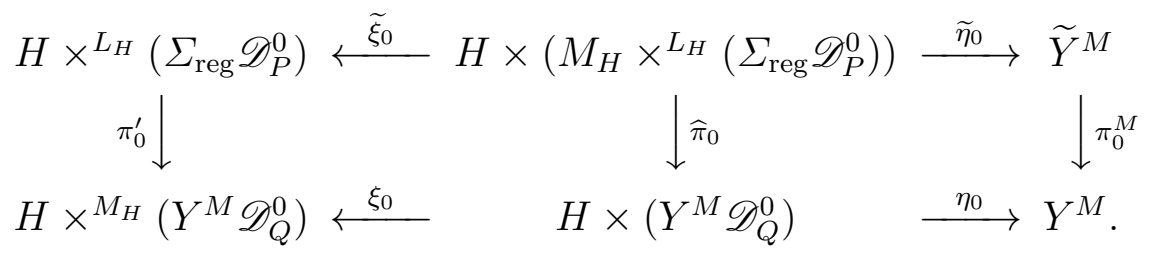

The maps are defined similarly to (6.4.4), and the diagram (6.4.10) enjoys similar properties as (6.4.4). In particular, the squares in the diagram are both cartesian. We consider the local system $\widetilde{\mathscr{E}}_{1}^{M}$ on $\widetilde{Y}^{M}$. Then one can check that the local system on $H \times{ }^{L_{H}}\left(\Sigma_{\text {reg }} \mathscr{D}_{P}^{0}\right)$, obtained from $\widetilde{\mathscr{E}}_{1}^{M}$ by a similar discussion as before, coincides with $\widetilde{\mathscr{E}}_{1}$ on $\widetilde{Y}$. It follows from (6.4.10) that the local system on $H \times{ }^{M_{H}}\left(Y^{M} \mathscr{D}_{Q}^{0}\right)$, obtained from the local system $\left(\pi_{0}^{M}\right)_{*} \widetilde{\mathscr{E}}_{1}^{M}$ on $Y^{M}$ by a similar discussion as before, coincides with $\left(\pi_{0}^{\prime}\right)_{*} \widetilde{\mathscr{E}}_{1}$. (6.4.9) now follows from this by applying the proper base change theorem to (6.4.4) and (6.4.10). Thus (6.4.2) is proved.

6.5. By applying the decomposition of $\mathscr{L}^{\prime}$ in (6.3.3) to the isomorphism in (6.4.2), we obtain the decomposition of $\pi_{*}^{\prime} K_{\overline{\mathscr{E}}_{1}}$ into simple perverse sheaves,

$$
\pi_{*}^{\prime} K_{\overline{\mathscr{E}}_{1}} \simeq \bigoplus_{\rho^{\prime} \in\left(\mathscr{A}_{\mathscr{E}_{1}^{\prime}}^{\prime}\right)^{\wedge}} \rho^{\prime} \otimes B_{\rho^{\prime}}
$$

where $B_{\rho^{\prime}} \simeq \operatorname{IC}\left(\widetilde{X}_{Q}, \mathscr{L}_{\rho^{\prime}}^{\prime}\right)\left[\operatorname{dim} \widetilde{X}_{Q}\right]$. By applying $\pi_{*}^{\prime \prime}$ on both sides of (6.5.1), we obtain

$$
\pi_{*} K_{\overline{\mathscr{E}}_{1}} \simeq \bigoplus_{\rho^{\prime} \in\left(\mathscr{A}_{\mathscr{E}_{1}}^{\prime}\right)^{\wedge}} \rho^{\prime} \otimes \pi_{*}^{\prime \prime} B_{\rho^{\prime}}
$$


Since $\pi^{\prime \prime}$ is proper, $\pi_{*}^{\prime \prime} B_{\rho^{\prime}}$ is a semisimple complex by the theorem of Deligne-Gabber. Since $\pi_{*}^{\prime \prime} B_{\rho^{\prime}}$ is a direct summand of a semisimple perverse sheaf $\pi_{*} K_{\overline{\mathscr{E}}_{1}}, \pi_{*}^{\prime \prime} B_{\rho^{\prime}}$ is a semisimple perverse sheaf, and all of its simple components have support on $X$. Since $\left.\pi_{*}^{\prime \prime} B_{\rho^{\prime}}\right|_{Y} \simeq\left(\pi_{0}^{\prime \prime}\right)_{*} \mathscr{L}_{\rho^{\prime}}^{\prime}[\operatorname{dim} X]$, we have

$$
\pi_{*}^{\prime \prime} B_{\rho^{\prime}} \simeq \operatorname{IC}\left(X,\left(\pi_{0}^{\prime \prime}\right)_{*} \mathscr{L}_{\rho^{\prime}}^{\prime}\right)[\operatorname{dim} X] .
$$

By applying the decomposition of $\left(\pi_{0}^{\prime \prime}\right)_{*} \mathscr{L}_{\rho^{\prime}}^{\prime}$ in $(6.3 .5)$, we obtain the following lemma, which is a perverse sheaf version of (6.3.5).

Lemma 6.6. For each $\rho^{\prime} \in\left(\mathscr{A}_{\mathscr{E}_{1}}^{\prime}\right)^{\wedge}, \rho \in \mathscr{A}_{\mathscr{E}_{1}}^{\wedge}$, put $A_{\rho}=\mathrm{IC}\left(X, \mathscr{L}_{\rho}\right)[\operatorname{dim} X], B_{\rho^{\prime}}=$ $\mathrm{IC}\left(\widetilde{X}_{Q}, \mathscr{L}_{\rho^{\prime}}^{\prime}\right)\left[\operatorname{dim} \widetilde{X}_{Q}\right]$. Then we have an isomorphism of perverse sheaves on $X$,

$$
\pi_{*}^{\prime \prime} B_{\rho^{\prime}} \simeq \bigoplus_{\rho \in \mathscr{A}_{\mathscr{E}_{1}}} A_{\rho} \otimes \overline{\mathbf{Q}}_{l}^{\left\langle\rho, \rho^{\prime}\right\rangle}
$$

6.7. Let $\left(\mathscr{O}_{\rho}, \mathscr{E}_{\rho}\right) \in \mathscr{N}_{G}$ be the pair which belongs to $\left(L \subset P, \mathscr{O}_{L}, \mathscr{E}_{1}^{\dagger}\right) \in \mathscr{S}_{G}$ and corresponds to $\rho \in \mathscr{A}_{\mathscr{E}_{1}}^{\wedge}$. Put $d=\operatorname{dim} X$, and let $d_{\rho}=d_{\mathscr{O}_{\rho}}$ be as in Theorem 5.2. By (5.2.2), we have $\left.A_{\rho}\right|_{X_{\text {uni }}} \simeq \operatorname{IC}\left(\overline{\mathscr{O}}_{\rho}, \mathscr{E}_{\rho}\right)\left[d-2 d_{\rho}\right]$, hence the following property holds. For any $\widetilde{\rho} \in \mathscr{A}_{\mathscr{E}_{1}}^{\wedge}$, we have

$$
\left.\mathscr{H}^{2 d_{\rho}-d} A_{\widetilde{\rho}}\right|_{\mathscr{O}_{\rho}} \text { is isomorphic to } \mathscr{E}_{\widetilde{\rho}} \text { if } \mathscr{O}_{\widetilde{\rho}}=\mathscr{O}_{\rho}
$$

Comparing (6.7.1) with Lemma 6.6, we have the following.

(6.7.2) $\left\langle\rho, \rho^{\prime}\right\rangle \leq$ the multiplicity of $\mathscr{E}_{\rho}$ in the local system $\left.\mathscr{H}^{2 d_{\rho}-d}\left(\pi_{*}^{\prime \prime} B_{\rho^{\prime}}\right)\right|_{\mathscr{O}_{\rho}}$.

Put $\left(\widetilde{X}_{Q}\right)_{\text {uni }}=\widetilde{X}_{Q} \cap\left(G_{\text {uni }}^{\iota \theta} \times H / Q_{H}\right)$. We will consider the restriction of $B_{\rho^{\prime}}$ on $\left(\widetilde{X}_{Q}\right)_{\text {uni }}$. Let $\left(\mathscr{O}_{\rho^{\prime}}^{\prime}, \mathscr{E}_{\rho^{\prime}}^{\prime}\right) \in \mathscr{N}_{M}$ be the pair which belongs to $\left(L \subset M \cap P,, \mathscr{O}_{L}, \mathscr{E}_{1}^{\dagger}\right) \in$ $\mathscr{S}_{M}$ and which corresponds to $\rho^{\prime} \in\left(\mathscr{A}_{\mathscr{E} 1}^{\prime}\right)^{\wedge}$. Put

$$
D=\left\{\left(x, g Q_{H}\right) \in G_{\text {uni }}^{c \theta} \times H / Q_{H} \mid g^{-1} x g \in \eta_{Q}^{-1}\left(\overline{\mathscr{O}}_{\rho^{\prime}}^{\prime}\right)\right\} .
$$

$D$ is a closed subvariety of $\left(\widetilde{X}_{Q}\right)_{\text {uni. }}$. We note that

$$
\left.\operatorname{supp} B_{\rho^{\prime}}\right|_{\left(\widetilde{X}_{Q}\right)_{\text {uni }}} \subset D
$$

In fact, as in(6.4.1), we denote by $A_{\rho^{\prime}}^{\prime}$ the simple perverse sheaf on $X^{M}$ appearing in the decomposition of $\left(\pi^{M}\right)_{*} K_{\overline{\mathscr{E}}_{1}^{M}}$, which corresponds to $\rho^{\prime} \in\left(\mathscr{A}_{\mathscr{E}_{1}}^{\prime}\right)^{\wedge}$. Then $B_{\rho^{\prime}}$ can be constructed from $A_{\rho^{\prime}}^{\prime}$ similarly to $K_{1}, K_{2}$, by using the diagram (6.4.4). On the other hand, by the generalized Springer correspondence for $M$, the restriction of $A_{\rho^{\prime}}^{\prime}$ on $M_{\text {uni }}^{\iota \theta}$ coincides with $\operatorname{IC}\left(\overline{\mathscr{O}}_{\rho^{\prime}}^{\prime}, \mathscr{E}_{\rho^{\prime}}\right)$, up to shift. (6.7.3) follows from this.

Let $\pi_{D}^{\prime \prime}$ be the restriction of $\pi^{\prime \prime}$ on $D$. In view of $(6.7 .3)$, (6.7.2) can be rewritten as follows.

(6.7.4) $\left\langle\rho, \rho^{\prime}\right\rangle \leq$ the multiplicity of $\mathscr{E}_{\rho}$ in the local system $\left.\mathscr{H}^{2 d_{\rho}-d}\left(\left(\pi_{D}^{\prime \prime}\right)_{!}\left(\left.B_{\rho^{\prime}}\right|_{D}\right)\right)\right|_{\mathscr{O}_{\rho}}$. 
Put $D_{0}=\left\{\left(x, g Q_{H}\right) \in G_{\text {uni }}^{\iota \theta} \times H / Q_{H} \mid g^{-1} x g \in \eta_{Q}^{-1}\left(\mathscr{O}_{\rho^{\prime}}^{\prime}\right)\right\}$. $D_{0}$ is an open subset of $D$. Let $\pi_{D_{0}}^{\prime \prime}$ be the restriction of $\pi^{\prime \prime}$ on $D_{0}$. We define an integer $x_{\rho, \rho^{\prime}}$ as the multiplicity of $\mathscr{E}_{\rho}$ in the local system $\left.\mathscr{H}^{2 d_{\rho}-d}\left(\left(\pi_{D_{0}}^{\prime \prime}\right)_{!}\left(\left.B_{\rho^{\prime}}\right|_{D_{0}}\right)\right)\right|_{\mathscr{O}_{\rho}}$. We show

Proposition 6.8. $\left\langle\rho, \rho^{\prime}\right\rangle$ coincides with $x_{\rho, \rho^{\prime}}$.

Proof. First we show that

$$
x_{r, \rho^{\prime}} \geq\left\langle\rho, \rho^{\prime}\right\rangle .
$$

Consider the long exact sequence of local systems obtained from the open embedding $D_{0} \hookrightarrow D$,

$$
\begin{aligned}
\cdots & \left.\longrightarrow \mathscr{H}^{2 d_{\rho}-d}\left(\left(\pi_{D_{0}}^{\prime \prime}\right)_{!}\left(\left.B_{\rho^{\prime}}\right|_{D_{0}}\right)\right)\right|_{\theta_{\rho}} \stackrel{\varphi}{\longrightarrow} \mathscr{H}^{2 d_{\rho}-d}\left(\left.\left(\pi_{D}^{\prime \prime}\right)_{!}\left(\left.B_{\rho^{\prime}}\right|_{D}\right)\right|_{\theta_{\rho}}\right. \\
& \left.\longrightarrow \mathscr{H}^{2 d_{\rho}-d}\left(\left(\pi_{D-D_{0}}^{\prime \prime}\right)_{!}\left(\left.B_{\rho^{\prime}}\right|_{D-D_{0}}\right)\right)\right|_{\theta_{\rho}} \longrightarrow \cdots
\end{aligned}
$$

If $\varphi$ is surjective, then (6.8.1) holds by (6.7.4). Thus it is enough to see that

$$
\left.\mathscr{H}^{2 d_{\rho}-d}\left(\left(\pi_{D-D_{0}}^{\prime \prime}\right)_{!}\left(\left.B_{\rho^{\prime}}\right|_{D-D_{0}}\right)\right)\right|_{\mathscr{O}_{\rho}}=0 .
$$

For this we have only to show that the stalk vanishes at any $x \in \mathscr{O}_{\rho}$. Hence we will show that

$$
\mathbf{H}_{c}^{2 d_{\rho}-d}\left(\left(\pi^{\prime \prime-1}(x) \cap\left(D-D_{0}\right), B_{\rho^{\prime}}\right)=0\right.
$$

for any $x \in \mathscr{O}_{\rho}$.

We consider the decomposition of $\overline{\mathscr{O}}_{\rho^{\prime}}^{\prime}$ into $M_{H^{-} \text {-orbits, }} \overline{\mathscr{O}}_{\rho^{\prime}}^{\prime}-\mathscr{O}_{\rho^{\prime}}^{\prime}=\coprod_{\alpha} \mathscr{O}_{\alpha}^{\prime}$. This implies the decomposition of $D-D_{0}$ into locally closed subsets, $D-D_{0}=\coprod_{\alpha} D_{\alpha}$, where

$$
D_{\alpha}=\left\{\left(x, g Q_{H}\right) \in G^{\iota \theta} \times H / Q_{H} \mid g^{-1} x g \in \eta_{Q}^{-1}\left(\mathscr{O}_{\alpha}^{\prime}\right)\right\} .
$$

In order to show (6.8.2), it is enough to see, for any $x \in \mathscr{O}_{\rho}$, and for any $D_{\alpha}$, that

$$
\mathbf{H}_{c}^{2 d_{\rho}-d}\left(\pi^{\prime \prime-1}(x) \cap D_{\alpha}, B_{\rho^{\prime}}\right)=0 .
$$

We remark that the following holds.

In fact, since the cohomology is non-zero, we have

$$
i \leq 2 \operatorname{dim}\left(\pi^{\prime \prime-1}(x) \cap D_{\alpha}\right) \leq\left(2 \nu_{H}-\operatorname{dim} \mathscr{O}_{\rho}\right)-\left(2 \nu_{M_{H}}-\operatorname{dim} \mathscr{O}_{\alpha}^{\prime}\right)+\Delta_{Q},
$$

where the second formula is obtained from Proposition 2.2 (ii), applied to $Q_{H} \subset H$. On the other hand, the condition $\left.\mathscr{H}^{j} B_{\rho^{\prime}}\right|_{D_{\alpha}} \neq 0$ is equivalent to the condition that 
$\left.\mathscr{H}^{j+\left(d-d^{\prime}\right)} A_{\rho^{\prime}}^{\prime}\right|_{\mathscr{O}_{\alpha}^{\prime}} \neq 0$ under the correspondence $B_{\rho^{\prime}} \leftrightarrow A_{\rho^{\prime}}^{\prime}$ through the diagram (6.4.4), where $d^{\prime}=\operatorname{dim} X^{M}$. Hence by applying (5.2.2) for $M$, we have

$$
\begin{aligned}
j+d & \leq\left(2 \nu_{M_{H}}-\operatorname{dim} \mathscr{O}_{\rho^{\prime}}^{\prime}\right)-\left(2 \nu_{L_{H}}-\operatorname{dim} \mathscr{O}_{L}\right)+\Delta_{P \cap M} \\
& <\left(2 \nu_{M_{H}}-\operatorname{dim} \mathscr{O}_{\alpha}^{\prime}\right)-\left(2 \nu_{L_{H}}-\operatorname{dim} \mathscr{O}_{L}\right)+\Delta_{P \cap M}
\end{aligned}
$$

Since $\Delta_{Q}+\Delta_{P \cap M}=\Delta_{P}$, we obtain (6.8.4).

Now by applying the hypercohomology spectral sequence, we obtain (6.8.3) from (6.8.4). Thus (6.8.2) holds, and so (6.8.1) is proved.

Since $\sum_{\rho^{\prime} \in\left(\mathscr{A}_{\mathscr{E}_{1}}^{\prime}\right)^{\wedge}}\left(\operatorname{dim} \rho^{\prime}\right)\left\langle\rho, \rho^{\prime}\right\rangle=\operatorname{dim} \rho$, in view of (6.8.1), in order to prove the proposition, it is enough to show the following formula.

$$
\sum_{\rho^{\prime} \in\left(\mathscr{A}_{\mathscr{E}_{1}}^{\prime}\right)^{\wedge}}\left(\operatorname{dim} \rho^{\prime}\right) x_{\rho, \rho^{\prime}}=\operatorname{dim} \rho .
$$

For an $M_{H^{-o r b i t}} \mathscr{O}^{\prime}$ in $M_{\text {uni }}^{\iota \theta}$, put $x_{\mathscr{O}^{\prime}}=\sum_{\rho^{\prime}}\left(\operatorname{dim} \rho^{\prime}\right) x_{\rho, \rho^{\prime}}$, where the sum is taken over all $\rho^{\prime} \in\left(\mathscr{A}_{\mathscr{E}_{1}^{\prime}}^{\prime}\right)^{\wedge}$ such that $\mathscr{O}_{\rho^{\prime}}^{\prime}=\mathscr{O}^{\prime}$. Then the sum in the left hand side of (6.8.5) coincides with $\sum_{\mathscr{O}^{\prime}} x_{\mathscr{O}^{\prime}}$, where the sum is taken over all $M_{H^{-o r b i t s}} \mathscr{O}^{\prime}$ in $M_{\text {uni }}^{\iota \theta}$. We consider $x_{\mathscr{O}^{\prime}}$ separately. It follows from the decomposition (6.5.1) and from the definition of $x_{\rho, \rho^{\prime}}$, we see that $x_{\mathscr{O}^{\prime}}$ coincides with the multiplicity of $\mathscr{E}_{\rho}$ in the local system

$$
\left.\mathscr{H}^{2 d_{\rho}-d}\left(\left(\pi_{D_{\mathscr{O}^{\prime}}^{\prime}}^{\prime \prime}\right) !\left(\left.\pi_{*}^{\prime} K_{\overline{\mathscr{E}}_{1}}\right|_{D_{\mathscr{O}^{\prime}}}\right)\right)\right|_{\mathscr{O}_{\rho}},
$$

where $D_{\mathscr{O}^{\prime}}$ is defined similarly to $D_{0}$ by replacing $\mathscr{O}_{\rho^{\prime}}^{\prime}$ by $\mathscr{O}^{\prime}$.

Here we note that the local system given in (6.8.6) coincides with the local system

$$
\left.\mathscr{H}^{2 d_{\rho}-2 d_{\rho^{\prime}}^{\prime}}\left(\left(\pi_{D_{\mathscr{O}^{\prime}}}^{\prime \prime}\right) !\left(\left.\mathscr{H}^{2 d_{\rho^{\prime}}^{\prime}-d}\left(\pi_{*}^{\prime} K_{\overline{\mathscr{E}}_{1}}\right)\right|_{D_{\mathscr{O}^{\prime}}}\right)\right)\right|_{\mathscr{O}_{\rho}}
$$

since the discussion in the proof of (6.8.4) shows that if $\left.\mathscr{H}^{i}\left(\left(\pi_{D_{\mathscr{O}^{\prime}}}^{\prime \prime}\right) ! \mathscr{F}\right)\right|_{\mathscr{O}_{\rho}} \neq 0$ for a sheaf $\mathscr{F}$, then $i \leq\left(2 \nu_{H}-\operatorname{dim} \mathscr{O}_{\rho}\right)-\left(2 \nu_{M_{H}}-\operatorname{dim} \mathscr{O}_{\rho^{\prime}}^{\prime}\right)+\Delta_{Q}=2 d_{\rho}-2 d_{\rho^{\prime}}^{\prime}$ and if $\left.\mathscr{H}^{j}\left(\pi_{*}^{\prime} K_{\overline{\mathscr{E}}_{1}}\right)\right|_{D_{\mathscr{O}^{\prime}}} \neq 0$, then $j \leq 2 d_{\rho^{\prime}}^{\prime}-d$.

By applying Theorem 5.2 (iii) to $M$, and by converting it to the sheaves on $\widetilde{X}_{0}$ by making use of the diagram (6.4.4), we obtain

$$
\left.\left.\mathscr{H}^{2 d_{\rho^{\prime}}^{\prime}-d}\left(\pi_{*}^{\prime} K_{\overline{\mathscr{E}}_{1}}\right)\right|_{D_{\mathscr{O}^{\prime}}} \simeq \mathscr{H}^{2 d_{\rho^{\prime}}^{\prime}}\left(\left.\pi^{\prime}\right|_{\widetilde{X}_{0}}\right) \overline{\mathscr{E}}_{1}\right|_{D_{\mathscr{O}^{\prime}}}
$$

where $\widetilde{X}_{0} \simeq H \times^{P_{H}} \eta_{P}^{-1}(\Sigma)$. Hence (6.8.7) can be rewritten as 


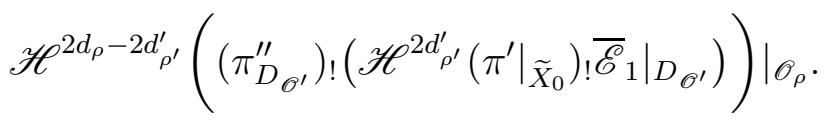

By using the spectral sequence associated to the composite $\pi=\pi^{\prime \prime} \circ \pi^{\prime}$, we see that the last formula is equal to

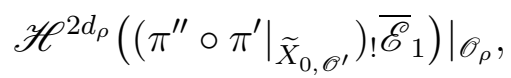

where $\widetilde{X}_{0, \mathscr{O}^{\prime}}=\widetilde{X}_{0} \cap \pi^{\prime-1}\left(D_{\mathscr{O}^{\prime}}\right)$. In particular, $x_{\mathscr{O}^{\prime}}$ coincides with the multiplicity of $\mathscr{E}_{\rho}$ in the local system given in (6.8.8).

Here we note that $\widetilde{X}_{0}=\coprod_{\mathscr{O}^{\prime}} \widetilde{X}_{0, \mathscr{O}^{\prime}}$ gives a partition of $\widetilde{X}_{0}$ by locally closed pieces $\widetilde{X}_{0, \mathscr{O}^{\prime}}$, where $\mathscr{O}^{\prime}$ runs over all the $M_{H^{-}}$orbits in $M_{\mathrm{uni}}^{\iota \theta}$. By Proposition 2.2 (ii), $\operatorname{dim}\left(\widetilde{X}_{0, \mathscr{O}^{\prime}} \cap \pi^{-1}(x)\right) \leq d_{\rho}$ for any $x \in \mathscr{O}_{\rho}$. Then by applying the cohomology long exact sequence of $\pi^{-1}(x)$, we see that $\sum_{\mathscr{O}^{\prime}} x_{\mathscr{O}^{\prime}}$ coincides with the multiplicity of $\mathscr{E}_{\rho}$ in the local system

$$
\left.\mathscr{H}^{2 d_{\rho}}\left(\left(\left.\pi\right|_{\widetilde{X}_{0}}\right) ! \overline{\mathscr{E}}_{1}\right)\right|_{\mathscr{O}_{\rho}}
$$

On the other hand, the stalk at $x \in \mathscr{O}_{\rho}$ of the local system in (6.8.9) coincides with $H_{c}^{2 d_{\rho}}\left(\mathscr{P}_{\mathscr{O}_{L}, x}, \overline{\mathscr{E}}_{1}\right)$, where $\mathscr{P}_{\mathscr{O}_{L}, x}=\left\{g P_{H} \in H / P_{H} \mid g^{-1} x g \in \eta_{P}^{-1}\left(\mathscr{O}_{L}\right)\right\}$. By Theorem 5.2, $H_{c}^{2 d_{\rho}}\left(\mathscr{P}_{\mathscr{O}_{L}, x}, \overline{\mathscr{E}}_{1}\right)$ has a natural structure of $\mathscr{A}_{\mathscr{E}_{1}} \times A_{H}(x)$-module, and is decomposed as

$$
H_{c}^{2 d_{\rho}}\left(\mathscr{P}_{\mathscr{O}_{L}, x}, \overline{\mathscr{E}}_{1}\right) \simeq \bigoplus_{\widetilde{\rho} \in \mathscr{A}_{\hat{\mathscr{E}}_{1}}} V_{\widetilde{\rho}} \otimes \tau_{\widetilde{\rho}}
$$

where $V_{\widetilde{\rho}}$ is an $\mathscr{A}_{\mathscr{E}_{1}}$-module, which is isomorphic to $\widetilde{\rho}$ if it is non-zero, and $\tau_{\widetilde{\rho}}$ is an irreducible representation of $A_{H}(x)$ corresponding to the local system $\mathscr{E} \widetilde{\rho}$ on $\mathscr{O}_{\rho}=\mathscr{O}_{\widetilde{\rho}}$. In particular, the multiplicity of $\mathscr{E}_{\rho}$ in the local system in (6.8.9) coincides with $\operatorname{dim} \rho$ if it is non-zero. But by $(6.8 .1)$, we know that $\sum_{\mathscr{O}^{\prime}} x_{\mathscr{O}^{\prime}} \geq \operatorname{dim} \rho$. It follows that $\sum_{\mathscr{O}^{\prime}} x_{\mathscr{O}^{\prime}}=\operatorname{dim} \rho$. Thus we have proved (6.8.5), and the proposition follows.

6.9. We are now ready to prove the theorem. For an $H$-orbit $\mathscr{O}$ in $G_{\text {uni }}^{\iota \theta}$ and an $M_{H^{-}}$orbit $\mathscr{O}^{\prime}$ in $M_{\text {uni }}^{\iota \theta}$, consider the diagram as in (5.3.1), but by replacing $P$ by $Q$, namely,

$$
\begin{gathered}
V=H \times{ }^{Q_{H}}\left(\mathscr{O} \cap \eta_{Q}^{-1}\left(\mathscr{O}^{\prime}\right)\right) \stackrel{f_{1}}{\longrightarrow} \mathscr{O} \\
f_{2} \downarrow \\
V^{\prime}=H \times{ }^{Q_{H}} \mathscr{O}^{\prime} .
\end{gathered}
$$


By putting

$$
\begin{aligned}
& d_{1}=\left(\nu_{H}-\operatorname{dim} \mathscr{O} / 2\right)-\left(\nu_{M_{H}}-\operatorname{dim} \mathscr{O}^{\prime} / 2\right)+\Delta_{Q} / 2, \\
& d_{2}=\left(\operatorname{dim} \mathscr{O}-\operatorname{dim} \mathscr{O}^{\prime}\right) / 2+\Delta_{Q} / 2 .
\end{aligned}
$$

as in 5.3, we can apply Lemma 5.4 for $Q$.

Since $\mathscr{O}^{\prime} \subset X^{M}$, we have $V \subset H \times{ }^{Q_{H}}\left(\eta_{Q}^{-1}\left(X^{M}\right)\right)$. The lower row of the diagram (6.4.4) gives a diagram, where $\xi_{1}, \eta_{1}$ are restrictions of $\xi, \eta$ on $H \times\left(\mathscr{O} \cap \eta_{Q}^{-1}\left(\mathscr{O}^{\prime}\right)\right)$.

$$
H \times{ }^{Q_{H}}\left(\mathscr{O} \cap \eta_{Q}^{-1}\left(\mathscr{O}^{\prime}\right)\right) \stackrel{\xi_{1}}{\longleftarrow} H \times\left(\mathscr{O} \cap \eta_{Q}^{-1}\left(\mathscr{O}^{\prime}\right)\right) \stackrel{\eta_{1}}{\longrightarrow} \mathscr{O}^{\prime}
$$

By using a similar argument as in 6.4 , we obtain an $H$-equivariant simple local system $\widetilde{\mathscr{E} \prime}$ on $V$ from an $H$-equivariant simple local system $\mathscr{E}^{\prime}$ on $\mathscr{O}^{\prime}$. On the other hand, $V^{\prime}$ is a single $H$-orbit, and its stabilizer is isomorphic to $Z_{M_{H}}(v) U_{Q_{H}}$ for $v \in \mathscr{O}^{\prime}$. Hence the set of $H$-equivariant simple local systems on $V^{\prime}$ is in bijection with the set of $M_{H}$-equivariant simple local systems on $\mathscr{O}^{\prime}$. We denote by $\widehat{\mathscr{E}}^{\prime}$ the local system on $V^{\prime}$ corresponding to $\mathscr{E}^{\prime}$ on $\mathscr{O}^{\prime}$. Here we note that

$$
f_{2}^{*} \widehat{\mathscr{E}}^{\prime} \simeq \widetilde{\mathscr{E} \prime}
$$

In fact, since both of $V, V^{\prime}$ are locally trivial fibration over $H / Q_{H}$, we have the following commutative diagram from the embedding of fibres.

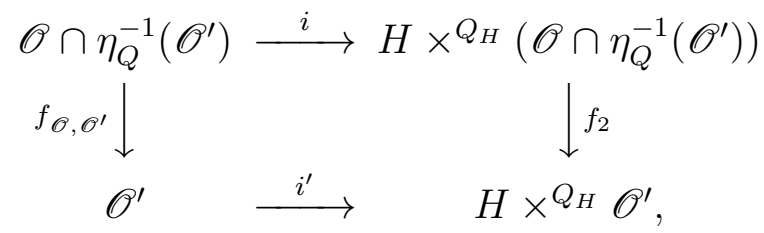

where $f_{\mathscr{O}, \mathscr{O}^{\prime}}$ is the map defined in 6.1 , and $i, i^{\prime}$ are inclusions of fibres. Since $f_{2}^{*} \widehat{\mathscr{E}}^{\prime}, \widetilde{\mathscr{E}}^{\prime}$ are both $H$-equivariant local systems, it is enough to show that their restrictions on $\mathscr{O} \cap \eta_{Q}^{-1}\left(\mathscr{O}^{\prime}\right)$ coincide. Since the restriction of $\widehat{\mathscr{E} \prime}$ on $\mathscr{O}^{\prime}$ is equal to $\mathscr{E}^{\prime}$, the restriction of $f_{2}^{*} \widehat{\mathscr{E}^{\prime}}$ coincides with $f_{\mathscr{O}, \mathscr{O}^{\prime}}^{*} \mathscr{E}^{\prime}$. On the other hand, it follows from the construction of $\widetilde{\mathscr{E} \prime}$, one can check that the restriction of $\widetilde{\mathscr{E}}^{\prime}$ on $\mathscr{O} \cap \eta_{Q}^{-1}\left(\mathscr{O}^{\prime}\right)$ coincides with $\mathscr{E} \prime$. Thus (6.9.1) holds.

Recall that $m_{\mathscr{E}, \mathscr{E}}$ is the multiplicity of $\mathscr{E}^{\prime}$ in the local system $R^{2 d_{\mathscr{O}, \mathscr{O}^{\prime}}}\left(f_{\mathscr{O}, \mathscr{O}^{\prime}}\right) ! \mathscr{E}$. Here $d_{2}=d_{\mathscr{O}, \mathscr{O}^{\prime}}$. Since $(6.9 .2)$ is the fibre product, we see that the restriction of $R^{2 d_{2}}\left(f_{2}\right) !\left(f_{1}^{*} \mathscr{E}\right)$ on $\mathscr{O}^{\prime}$ coincides with $R^{2 d_{\mathscr{O}, \mathscr{O}^{\prime}}}\left(f_{\mathscr{O}, \mathscr{O}^{\prime}}\right) ! \mathscr{E}$. Hence $m_{\mathscr{E}, \mathscr{E}}$ coincides with the multiplicity of $\widehat{\mathscr{E}}^{\prime}$ in the local system $R^{2 d_{2}}\left(f_{2}\right) !\left(f_{1}^{*} \mathscr{E}\right)$. Then by using (6.9.1) and Lemma 5.4, we obtain

$$
m_{\mathscr{E}, \mathscr{E} \prime} \text { coincides with the multiplicity of } \mathscr{E} \text { in the local system } R^{2 d_{1}}\left(f_{1}\right), \widetilde{\mathscr{E}}^{\prime}
$$


Here the perverse sheaf $B_{\rho^{\prime}}$ on $\widetilde{X}_{Q}$ is constructed from $A_{\rho^{\prime}}^{\prime}$ on $X^{M}$ by using the diagram (6.4.4). The local system $\widetilde{\mathscr{E}}^{\prime}$ on $V$ is also constructed from $\mathscr{E}^{\prime}$ on $\mathscr{O}^{\prime}$ by (6.4.4). If we write $\left(\mathscr{O}^{\prime}, \mathscr{E}^{\prime}\right)=\left(\mathscr{O}_{\rho^{\prime}}^{\prime}, \mathscr{E}_{\rho^{\prime}}^{\prime}\right)$, one can check that $\left.\mathscr{H}^{2 d_{\rho^{\prime}}^{\prime}-d} B_{\rho^{\prime}}\right|_{V} \simeq \widetilde{\mathscr{E}^{\prime}}$. Hence by $(6.9 .3), m_{\mathscr{E}, \mathscr{E}^{\prime}}$ coincides with the multiplicity of $\mathscr{E}$ in the local system $\left.R^{2 d_{1}}\left(\left.\pi^{\prime \prime}\right|_{V}\right)_{!}\left(\left.\mathscr{H}^{2 d^{\prime}{ }^{\prime}-d} B_{\rho^{\prime}}\right|_{V}\right)\right|_{\mathscr{O}}$. If we write $(\mathscr{O}, \mathscr{E})=\left(\mathscr{O}_{\rho}, \mathscr{E}_{\rho}\right)$, we have $d_{1}=d_{\rho}-d_{\rho^{\prime}}^{\prime}$. It follows from the definition of $x_{\rho, \rho^{\prime}}$ and (6.8.7), that this multiplicity of $\mathscr{E}$ actually coincides with $x_{\rho, \rho^{\prime}}$. Hence by Proposition 6.8, we conclude that $m_{\mathscr{E}, \mathscr{E}^{\prime}}=\left\langle\rho, \rho^{\prime}\right\rangle$. This proves (ii) of the theorem.

We show (i). Take $\left(\mathscr{O}^{\prime}, \mathscr{E}^{\prime}\right) \in \mathscr{N}_{M}$ which belongs to $\left(L \subset M \cap P, \mathscr{O}_{L}, \mathscr{E}_{1}^{\dagger}\right)$. Assume that $m_{\mathscr{E}, \mathscr{E}^{\prime}} \neq 0$. Since $\left.\mathscr{H}^{2 d_{\rho^{\prime}}^{\prime}-d} \pi_{*}^{\prime} K_{\overline{\mathscr{E}}_{1}}\right|_{V}$ contains $\widetilde{\mathscr{E}}^{\prime}$, the above discussion implies that $\mathscr{E}$ appears in the local system $\left.R^{2 d_{1}}\left(\left.\pi^{\prime \prime}\right|_{V}\right) ! R^{2 d^{\prime}{ }^{\prime}}\left(\left.\pi^{\prime}\right|_{\widetilde{X}_{0, \mathscr{O}}}\right) !\left(\overline{\mathscr{E}}_{1}\right)\right|_{V}$ on $\mathscr{O}$ with nonzero multiplicity. This is equivalent to saying that $\mathscr{E}$ appears in the local system

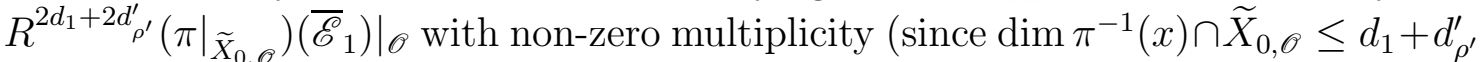
for $x \in \mathscr{O})$. It follows that $\mathscr{E}$ appears in the local system $\left.R^{2 d_{1}+2 d_{\rho^{\prime}}^{\prime}}\left(\left.\pi\right|_{\widetilde{X}_{0}}\right)\left(\overline{\mathscr{E}}_{1}\right)\right|_{\mathscr{O}}$ with non-zero multiplicity, by considering the partition of $\tilde{X}_{0}$ by locally closed pieces $\widetilde{X}_{0, \mathscr{O}^{\prime}}$. Now (i) follows from Theorem 5.2 (iii) since $d_{1}+d_{\rho^{\prime}}^{\prime}=d_{\mathscr{O}}$ in the notation there. This complete the proof of Theorem 6.2.

6.10. By making use of the discussion in 2.3, we reformulate the restriction theorem in a more convenient form for the application. We keep the notation in 6.1. Let $(\mathscr{O}, \mathscr{E}) \in \mathscr{N}_{G}$ and $\left(\mathscr{O}^{\prime}, \mathscr{E}^{\prime}\right) \in \mathscr{N}_{M}$. We fix $u \in \mathscr{O}, v \in \mathscr{O}^{\prime}$. Let $\tau \in A_{H}(u)^{\wedge}$ be the irreducible representation corresponding to $\mathscr{E}$, and $\tau^{\prime} \in A_{M_{H}}(v)^{\wedge}$ the irreducible representation corresponding to $\mathscr{E}^{\prime}$. As in 2.3 , but by replacing $L$ by $M$, we define varieties

$$
\begin{aligned}
& Y_{u, v}=\left\{g Z_{M_{H}}^{0}(v) U_{Q}^{\theta} \mid g \in H, g^{-1} u g \in \eta_{Q}^{-1}(v)\right\} \\
& \widetilde{Y}_{u, v}=\left\{g \in H \mid g^{-1} u g \in \eta_{Q}^{-1}(v)\right\}
\end{aligned}
$$

$Z_{H}(u) \times Z_{M_{H}}(v)$ acts on $Y_{u, v}$ by $\left(g_{1}, g_{2}\right): g Z_{M_{H}}^{0}(v) U_{Q}^{\theta} \mapsto g_{1} g g_{2}^{-1} Z_{M_{H}}^{0} U_{Q}^{\theta}$. By the discussion in 2.3 , we have

$$
\operatorname{dim} Y_{u, v} \leq s=\left(\operatorname{dim} Z_{H}(u)-\operatorname{dim} Z_{M_{H}}(v)\right) / 2+\Delta_{Q} / 2 .
$$

Let $I\left(Y_{u, v}\right)$ be the set of irreducible components in $Y_{u, v}$ of dimension $s$. Then $A_{H}(u) \times$ $A_{M_{H}}(v)$ acts on $I\left(Y_{u, v}\right)$ as the permutations. We denote by $\varepsilon_{u, v}$ the corresponding permutation representation of $A_{H}(u) \times A_{M_{H}}(v)$. As a corollary to the restriction theorem, we have the following result (cf $[\mathrm{LS}, 0.4]$ ).

Corollary 6.11. Assume that $(\mathscr{O}, \mathscr{E}) \in \mathscr{N}_{G}$ corresponds to $\rho \in \mathscr{A}_{\mathscr{E} 1}^{\wedge}$ and $\left(\mathscr{O}^{\prime}, \mathscr{E}^{\prime}\right) \in$ $\mathscr{N}_{M}$ corresponds to $\rho^{\prime} \in\left(\mathscr{A}_{\mathscr{E}_{1}}^{\prime}\right)^{\wedge}$. Then

$$
\left\langle\left.\rho\right|_{\mathscr{A}_{\mathscr{E}_{1}}^{\prime}} ^{\prime}, \rho^{\prime}\right\rangle_{\mathscr{A}_{\mathscr{E}_{1}}^{\prime}}=\left\langle\tau \otimes \tau^{\prime *}, \varepsilon_{u, v}\right\rangle_{A_{H}(u) \times A_{M_{H}}(v)} .
$$


Proof. Assume that $I\left(Y_{u, v}\right) \neq \emptyset$. Then it follows from the discussion in 2.3 that $\operatorname{dim}\left(\mathscr{O} \cap \eta_{Q}^{-1}(v)\right)=d_{\mathscr{O}, \mathscr{O}^{\prime}}=\left(\operatorname{dim} \mathscr{O}-\operatorname{dim} \mathscr{O}^{\prime}\right) / 2+\Delta_{Q} / 2$. As in 6.1 , let $m_{\mathscr{E}, \mathscr{E}^{\prime}}$ be the multiplicity of $\mathscr{E}^{\prime \prime}$ in the local system $R^{2 d_{\mathscr{O}, \mathscr{O}^{\prime}}}\left(f_{\mathscr{O}, \mathscr{O}^{\prime}}\right) ! \mathscr{E}$. Then $m_{\mathscr{E}, \mathscr{E}}$ coincides with the multiplicity of $\tau^{\prime *}$ in the $A_{M_{H}}(v)$-module $H_{c}^{2 d_{\mathscr{O}, \mathscr{O}^{\prime}}}\left(\mathscr{O} \cap \eta_{Q}^{-1}(v), \mathscr{E}\right)$. We consider a similar diagram as in (2.3.1). We use the same notation there. $Z_{H}(u) \times Z_{M_{H}}(v)$ acts on $\tilde{Y}_{u, v}, Z_{H}^{0}(u) \backslash \widetilde{Y}_{u, v}$, and $\phi, \psi$ are $Z_{H}(u) \times Z_{M_{H}}(v)$-equivariant. On the other hand, let $\xi: \widetilde{\mathscr{O}}=H / Z_{H}^{0}(u) \rightarrow \mathscr{O}=H / Z_{H}(u)$ be the natural map. Then $Z_{M_{H}}(v)$ acts on $\xi^{-1}\left(\mathscr{O} \cap \eta_{Q}^{-1}(v)\right)$ under the isomorphism $\xi^{-1}\left(\mathscr{O} \cap \eta_{Q}^{-1}(v)\right) \simeq Z_{H}^{0}(u) \widetilde{Y}_{u, v}$, and the map $\xi^{-1}\left(\mathscr{O} \cap \eta_{Q}^{-1}(v)\right) \rightarrow\left(\mathscr{O} \cap \eta_{Q}^{-1}(v)\right)$ becomes $Z_{M_{H}}$-equivariant. Under this situation, we can identify $H_{c}^{2 d_{\mathscr{E}, \mathscr{E}^{\prime}}}\left(\mathscr{O} \cap \eta_{Q}^{-1}(v), \mathscr{E}\right)$ with the multiplicity space of $\tau$ in the $A_{H}(u)$-module $H_{c}^{2 d_{\mathscr{O}, \mathscr{O}^{\prime}}}\left(\xi^{-1}\left(\mathscr{O} \cap \eta_{Q}^{-1}(v)\right), \overline{\mathbf{Q}}_{l}\right)$. Moreover, this identification is compatible with the action of $A_{M_{H}}(v)$ on both cohomologies. It follows that $m_{\mathscr{E}, \mathscr{E}^{\prime}}$ coincides with the multiplicity of $\tau \otimes \tau^{*}$ in the $A_{H}(u) \times A_{M_{H}}(v)$-module $H_{c}^{2 d_{\mathscr{O}, \mathscr{O}^{\prime}}}\left(\xi^{-1}\left(\mathscr{O} \cap \eta_{Q}^{-1}(v)\right), \overline{\mathbf{Q}}_{l}\right)$. From the discussion in 2,3 , this $A_{H}(u) \times A_{M_{H}}(v)$ module is isomorphic to the permutation representation $\varepsilon_{u, v}$ on the set $I\left(Y_{u, v}\right)$. Thus the corollary follows from Theorem 6.2.

\section{UNIPOTENT ORBITS}

7.1. We follow the notation in 1.7. Since $\mathfrak{g}^{-}$is the set of self-adjoint matrices in $\mathfrak{g}=\mathfrak{g l}_{N}$, and $G^{\iota \theta}$ is the set of non-degenerate self-adjoint matrices in $\mathfrak{g}$, the map $x \mapsto x-1$ gives an isomorphism between $G_{\text {uni }}^{\iota \theta}$ and $\mathfrak{g}_{\text {nil }}^{-}$. This map is clearly $H$ equivariant. In this way, we have a natural bijection between the set of $H$-orbits in $G_{\text {uni }}^{\iota \theta}$ and the set of $H$-orbits in $\mathfrak{g}_{\text {nil }}^{-}$.

For an integer $m \geq 0$, let $\mathscr{P}_{m}$ be the set of partitions $\lambda=\left(\lambda_{1}, \ldots, \lambda_{r}\right)$ with $\lambda_{r} \geq 0$ such that $\sum_{i} \lambda_{i}=m . \quad m=|\lambda|$ is called the size of $\lambda$, and the maximal number $k=\ell(\lambda)$ such that $\lambda_{k} \neq 0$ is called the length of $\lambda$. For $\lambda=\left(\lambda_{1}, \ldots, \lambda_{r}\right)$, put

$$
n(\lambda)=\sum_{i=1}^{r}(i-1) \lambda_{i} .
$$

It is known that the set of unipotent conjugacy classes in $G=G L_{N}$ is in bijection with $\mathscr{P}_{N}$, via Jordan normal form. We denote by $\mathbb{O}_{\lambda}$ the unipotent class in $G$ corresponding to $\lambda \in \mathscr{P}_{N}$. Similarly, the set of nilpotent orbits in $\mathfrak{g}_{\text {nil }}$ is in bijection with $\mathscr{P}_{N}$. We also denote by $\mathbb{O}_{\lambda}$ the corresponding nilpotent orbit in $\mathfrak{g}_{\text {nil }}$, if there is no fear of confusion. For $x \in \mathbb{O}_{\lambda} \subset \mathfrak{g}_{\text {nil }}$, we define a Jordan basis $\left\{v_{i, j} \mid 1 \leq i \leq \ell(\lambda), 1 \leq j \leq \lambda_{i}\right\}$ of $V$ with respect to $x$ by $x v_{i, j}=v_{i, j-1}$ if $j \geq 2$, and $x v_{i, 1}=0$. The following result gives a parametrization of $G^{\theta}$-orbits in $G_{\text {uni }}^{\iota \theta}$.

Lemma 7.2. For any $\lambda \in \mathscr{P}_{N}, \mathbb{O}_{\lambda} \cap G_{\text {uni }}^{\iota \theta} \neq \emptyset$. Any two elements in $\mathbb{O}_{\lambda} \cap G_{\text {uni }}^{\iota \theta}$ is conjugate under $G^{\theta}$. Thus the set of $G^{\theta}$-orbits in $G_{\mathrm{uni}}^{\mathrm{c}}$ is in bijection with $\mathscr{P}_{N}$. 
Proof. It is enough to prove the corresponding fact for $\mathfrak{g}_{\text {nil }}^{-}$. The argument below is in part due to [CVX1, Lemma 2.2]. First assume that $\mathbb{O}_{\lambda} \cap \mathfrak{g}_{\text {nil }}^{-} \neq \emptyset$. Take $x \in \mathbb{O}_{\lambda} \cap \mathfrak{g}_{\text {nil }}^{-}$, with $\lambda=\left(\lambda_{1}, \ldots, \lambda_{r}\right)$. We show that there exists a Jordan basis $\left\{v_{i, j}\right\}$ of $V$ with respect to $x$ satisfying the following property;

$$
\left\langle v_{i, j}, v_{i^{\prime}, j^{\prime}}\right\rangle= \begin{cases}1 & \text { if } i=i^{\prime} \text { and } j+j^{\prime}=\lambda_{i}+1 \\ 0 & \text { otherwise. }\end{cases}
$$

Since $\left\langle\operatorname{Ker} x^{\lambda_{1}-1}, \operatorname{Im} x^{\lambda_{1}-1}\right\rangle=0$, there exists $v_{1} \in V$ such that $\left\langle v_{1}, x^{\lambda_{1}-1} v_{1}\right\rangle \neq 0$. We may assume that $\left\langle v_{1}, x^{\lambda_{1}-1} v_{1}\right\rangle=1$. Then we have $\left\langle x^{i} v_{1}, x^{j} v_{1}\right\rangle=0$ unless $i+j=$ $\lambda_{1}-1$, in which case it is equal to 1 . Let $W_{1}$ be the subspace of $V$ spanned by $v_{1}, x v_{1}, \ldots, x^{\lambda_{1}-1} v_{1}$. Then $\operatorname{dim} W_{1}=\lambda_{1}$, and the restriction of the bilinear form $\langle$, on $W_{1}$ is non-degenerate. Hence we can write as $V=W_{1} \oplus W_{1}^{\perp}$, and $W_{1}^{\perp}$ is stable by $x$. The Jordan type of $\left.x\right|_{W_{1}^{\perp}}$ is $\lambda^{\prime}=\left(\lambda_{2}, \ldots, \lambda_{r}\right)$. Thus by induction on $N=\operatorname{dim} V$, one can find a Jordan basis $\left\{v_{i, j}\right\}$ satisfying the property (7.2.1).

If we take another $x^{\prime} \in \mathbb{O}_{\lambda} \cap \mathfrak{g}_{\text {nil }}^{-}$, we can find a Jordan basis $\left\{v_{i, j}^{\prime}\right\}$ satisfying (7.2.1). The map $g: v_{i j} \mapsto v_{i, j}^{\prime}$ determines $g \in G^{\theta}$, and we have $g x=x^{\prime}$. Thus $\mathbb{O}_{\lambda} \cap \mathfrak{g}_{\text {nil }}^{-}$consists of a single $G^{\theta}$-orbit.

Next we show that $\mathbb{O}_{\lambda} \cap \mathfrak{g}_{\text {nil }}^{-} \neq \emptyset$. Take $x \in \mathbb{O}_{\lambda} \subset \mathfrak{g}_{\text {nil }}$, and choose a Jordan basis $\left\{v_{i j}\right\}$ of $V$ with respect to $x$. We define a symmetric bilinear form $\langle,\rangle^{\prime}$ on $V$ by a similar condition as in (7.2.1). Then $x$ is self-adjoint with respect to this bilinear form, namely, we have $\langle x v, w\rangle^{\prime}=\langle v, x w\rangle^{\prime}$ for any $v, w \in V$. By 1.7, this implies that some $G$-conjugate of $x$ is contained in $\mathfrak{g}_{\text {nil }}^{-}$. Hence $\mathbb{O}_{\lambda} \cap \mathfrak{g}_{\text {nil }}^{-} \neq \emptyset$. The lemma is proved.

7.3. We denote by $\mathscr{O}_{\lambda}$ the $G^{\theta}$-orbit in $G_{\text {uni }}^{\iota \theta}$ corresponding to $\lambda \in \mathscr{P}_{N}$. Also by the same symbol $\mathscr{O}_{\lambda}$, we denote the $G^{\theta}$-orbit in $\mathfrak{g}_{\text {nil }}^{-}$. For $x \in \mathscr{O}_{\lambda} \subset \mathfrak{g}_{\text {nil }}^{-}$, a Jordan basis $\left\{v_{i, j}\right\}$ of $V$ with respect to $x$ satisfying the property in (7.2.1) is called a normal basis of $x$.

We shall compute $\operatorname{dim} Z_{G^{\theta}}(x)$ for $x \in \mathscr{O}_{\lambda}$. First we recall the well-known result in the case of $Z_{G}(x)$ for $x \in \mathbb{O}_{\lambda}$. Take $x \in \mathbb{O}_{\lambda} \subset \mathfrak{g}_{\text {nil }}$, and put $E^{x}=\{y \in \operatorname{End}(V) \mid$ $x y=y x\}$. Note that $\operatorname{dim} Z_{G}(x)=\operatorname{dim} E^{x}$. Let $\left\{v_{i, j}\right\}$ be a Jordan basis of $V$ with respect to $x$. Following [AH, Proposition 2.8], we define $y_{i_{1}, i_{2}, s} \in \operatorname{End}(V)$ as follows;

$$
y_{i_{1}, i_{2}, s}\left(v_{i, j}\right)= \begin{cases}v_{i_{2}, j-s} & \text { if } i=i_{1}, s+1 \leq j \leq \lambda_{i} \\ 0 & \text { otherwise }\end{cases}
$$

Then

$$
\left\{y_{i_{1}, i_{2}, s} \mid i_{1}, i_{2} \leq \ell(\lambda), \max \left\{0, \lambda_{i_{1}}-\lambda_{i_{2}}\right\} \leq s \leq \lambda_{i_{1}}-1\right\}
$$

gives rise to a basis of the $\mathbf{k}$-vector space $E^{x}$. Hence we have

$$
\operatorname{dim} E^{x}=\sum_{i_{1} \geq i_{2}} \lambda_{i_{2}}+\sum_{i_{1}<i_{2}} \lambda_{i_{1}}=N+2 n(\lambda) .
$$

We show a lemma. 
Lemma 7.4. For $x \in \mathscr{O}_{\lambda} \subset G_{\text {uni }}^{\iota \theta}$, we have $\operatorname{dim} Z_{G^{\theta}}(x)=n(\lambda)$.

Proof. It is enough to show the corresponding fact for $x \in \mathscr{O}_{\lambda} \subset \mathfrak{g}_{\text {nil }}^{-}$. Thus we have only to show that $\operatorname{dim} Z_{\mathfrak{g}^{+}}(x)=n(\lambda)$. Here $E^{x}=Z_{\mathfrak{g}}(x)$ is stable under $\theta$, and we can decompose $E^{x}=\left(E^{x}\right)^{+} \oplus\left(E^{x}\right)^{-}$, where $\left(E^{x}\right)^{+}=Z_{\mathfrak{g}^{+}}(x)$. We choose a normal basis of $x$, and define $y_{i_{1}, i_{2}, s}$ by using this basis. Then by 1.7 ,

$$
\left\{y_{i_{1}, i_{2}, s}+y_{i_{2}, i_{1}, s+\left(\lambda_{i_{2}}-\lambda_{i_{1}}\right)} \mid i_{1} \leq i_{2}, 0 \leq s \leq \lambda_{i_{1}}-1\right\}
$$

gives a basis of $\left(E^{x}\right)^{-}$and

$$
\left\{y_{i_{1}, i_{2}, s}-y_{i_{2}, i_{1}, s+\left(\lambda_{i_{2}}-\lambda_{i_{1}}\right)} \mid i_{1}<i_{2}, 0 \leq s \leq \lambda_{i_{1}}-1\right\}
$$

gives a basis of $\left(E^{x}\right)^{+}$. It follows that

$$
\operatorname{dim}\left(E^{x}\right)^{+}=n(\lambda), \quad \operatorname{dim}\left(E^{x}\right)^{-}=n(\lambda)+N .
$$

The lemma follows from this.

Remark 7.5. In the case where $\mathbf{k}=\mathbf{C}$, the lemma follows from the general result of Kostant-Rallis [KR]. In fact, by [KR, Proposition 5], we have

$$
\operatorname{dim}\left[\mathfrak{g}^{+}, x\right]=\operatorname{dim}[\mathfrak{g}, x] / 2
$$

for $x \in \mathfrak{g}^{-}$. Now assume that $x \in \mathscr{O}_{\lambda}$. By (7.3.1), $\operatorname{dim}[\mathfrak{g}, x]=\operatorname{dim} \mathbb{O}_{\lambda}=N^{2}-(N+$ $2 n(\lambda)$ ). If we notice that $\operatorname{dim} G^{\theta}=\left(N^{2}-N\right) / 2,(7.5 .1)$ implies that $\operatorname{dim}\left[\mathfrak{g}^{+}, x\right]=$ $\operatorname{dim} \mathscr{O}_{\lambda}=\operatorname{dim} G^{\theta}-n(\lambda)$, and the lemma follows.

7.6. Take $x \in \mathscr{O}_{\lambda} \subset \mathfrak{g}_{\text {nil }}^{-}$. We write $\lambda \in \mathscr{P}_{N}$ as $\lambda=\left(a_{1}^{m_{1}}, a_{2}^{m_{2}}, \ldots, a_{h}^{m_{h}}\right)$ with $a_{1}>a_{2}>\cdots>a_{h}>0$. Then by using a normal basis of $x$, we have a decomposition of $V$ into $x$-stable subspaces

$$
V=\bigoplus_{1 \leq i \leq h} \widetilde{J}_{i}
$$

where $\widetilde{J}_{i}$ 's are mutually orthogonal, and the restriction of $x$ on $\widetilde{J}_{i}$ has the Jordan type $\left(a_{i}^{m_{i}}\right)$. By this decomposition, we have an embedding of groups

$$
\prod_{1 \leq i \leq h} G_{i}^{\theta} \subset G^{\theta}
$$

where $G_{i}=G L\left(\widetilde{J}_{i}\right)$ is a $\theta$-stable subgroup of $G$. One can find a subspace $J_{i}$ of $\widetilde{J}_{i}$ such that $\operatorname{dim} J_{i}=m_{i}$ and that $\widetilde{J}_{i}=J_{i} \oplus x J_{i} \oplus \cdots \oplus x^{a_{i}-1} J_{i}$. We define a quadratic form $Q_{i}$ on $J_{i}$ by 


$$
Q_{i}(v)=\left\langle v, x^{a_{i}-1} v\right\rangle, \quad\left(v \in J_{i}\right) .
$$

Then $Q_{i}$ is non-degenerate on $J_{i}$. Let $O_{i}=O\left(J_{i}\right)$ be the orthogonal group on $J_{i}$ with respect to the quadratic form $Q_{i}$. For each $g \in O_{i}$, we define a map $\widetilde{g}: \widetilde{J}_{i} \rightarrow \widetilde{J}_{i}$ by $\widetilde{g}\left(x^{k} v\right)=x^{k}(g v)$ for $0 \leq k \leq a_{i}-1, v \in J_{i}$. Then $\widetilde{g}$ commutes with the action of $x$ on $\widetilde{J}_{i}$, and $\widetilde{g}$ preserves the bilinear form $\langle$,$\rangle on \widetilde{J}_{i}$. Hence $\widetilde{g} \in Z_{G_{i}^{\theta}}(x)$. It is clear that $g \mapsto \widetilde{g}$ gives a homomorphism $O_{i} \rightarrow Z_{G_{i}^{\theta}}(x)$ whose image is isomorphic to $O_{i}$. Thus we can construct a closed subgroup of $Z_{G^{\theta}}(x)$ which is isomorphic to $\prod_{1 \leq i \leq h} O_{i}$. Actually, this subgroup is the reductive part of $Z_{G^{\theta}}(x)$, and, as in the classical case, one can show that

$$
Z_{G^{\theta}}(x) \simeq\left(\prod_{1 \leq i \leq h} O_{i}\right) \ltimes U_{1},
$$

where $U_{1}$ is a connected unipotent normal subgroup of $Z_{G^{\theta}}(x)$.

7.7 Put $A_{G^{\theta}}(x)=Z_{G^{\theta}}(x) / Z_{G^{\theta}}^{0}(x)$ for $x \in \mathscr{O}_{\lambda}$. Then by (7.6.4), $A_{G^{\theta}}(x) \simeq$ $(\mathbf{Z} / 2 \mathbf{Z})^{h}$. We write an element $\alpha \in A_{G^{\theta}}(x)$ as $\alpha=\left(\alpha_{1}, \ldots, \alpha_{h}\right)$ with $\alpha_{i}= \pm 1$, where $\alpha_{i}=-1$ is the generator of the $i$-th component $\mathbf{Z} / 2 \mathbf{Z}$. Let $A_{H}(x)=Z_{H}(x) / Z_{H}^{0}(x)$ as before. Since $Z_{G^{\theta}}^{0}(x)=Z_{H}^{0}(x), A_{H}(x)$ is a subgroup of $A_{G^{\theta}}(x)$. It follows from the discussion in 7.6 , we see easily that

$$
A_{H}(x)=\left\{\alpha=\left(\alpha_{1}, \ldots, \alpha_{h}\right) \in A_{G^{\theta}}(x) \mid \prod_{a_{i}: \text { odd }} \alpha_{i}=1\right\} .
$$

A partition $\lambda=\left(\lambda_{1}, \ldots, \lambda_{r}\right)$ is called an even partition if $\lambda_{i}$ is even for each $i$. Then (7.7.1) can be written as

$$
A_{H}(x) \simeq \begin{cases}(\mathbf{Z} / 2 \mathbf{Z})^{h} & \text { if } \lambda \text { is even, } \\ (\mathbf{Z} / 2 \mathbf{Z})^{h-1} & \text { if } \lambda \text { is not even }\end{cases}
$$

Proposition 7.8. $\quad$ (i) Assume that $N$ is odd. Then each $G^{\theta}$-orbit $\mathscr{O}_{\lambda}$ is a single $H$-orbit.

(ii) Assume that $N$ is even. If $\lambda$ is not an even partition, $\mathscr{O}_{\lambda}$ is a single $H$-orbit. If $\lambda$ is an even partition, $\mathscr{O}_{\lambda}$ splits into two $H$-orbits.

Proof. Since $G^{\theta}=H \cup H \zeta$ for some $\zeta \in G^{\theta}-H$, we have $G^{\theta} x=H x \cup H(\zeta x)$. Thus $G^{\theta} x=H x$ if $\zeta \in Z_{G^{\theta}}(x)$, namely, if $Z_{G^{\theta}}(x) \supsetneq Z_{H}(x)$. If $N$ is odd, $-1 \in$ $Z_{G^{\theta}}(x)-Z_{H}(x)$ satisfies this condition. If $N$ is even and $\lambda$ is not an even partition, then $Z_{G^{\theta}}(x) \supsetneq Z_{H}(x)$ by (7.7.2). Hence $\mathscr{O}_{\lambda}$ is a single $H$-orbit. Now assume that $N$ is even and $\lambda$ is an even partition. In this case, $Z_{G^{\theta}}(x)=Z_{H}(x)$. We have

$$
G^{\theta} / Z_{G^{\theta}}(x) \simeq(H \coprod \zeta H) / Z_{H}(x) \simeq H / Z_{H}(x) \coprod \zeta H / Z_{H}(x) .
$$


Hence $\mathscr{O}_{\lambda}$ splits into two $H$-orbits.

7.9. Let $\mathbb{O}_{\lambda}$ be the regular unipotent class in $G_{\text {uni }}$, where $\lambda=(N) \in \mathscr{P}_{N}$. Then $\mathbb{O}_{\lambda}$ is open dense in $G_{\text {uni }}$. Since $\mathbb{O}_{\lambda} \cap G_{\text {uni }}^{\iota \theta} \neq \emptyset, \mathbb{O}_{\lambda} \cap G_{\text {uni }}^{\iota \theta}$ is an open dense subset of $G_{\text {uni }}^{\iota \theta}$. In the case where $N$ is odd, $\mathbb{O}_{\lambda} \cap G_{\text {uni }}^{\iota \theta}=\mathscr{O}_{\lambda}$ is a single $H$-orbit. Thus $\mathscr{O}_{\lambda}$ is the unique $H$-orbit in $G_{\text {uni }}^{\iota \theta}$ such that $G_{\text {uni }}^{\iota \theta}=\overline{\mathscr{O}}_{\lambda}$. In particular, $G_{\text {uni }}^{\iota \theta}$ is an irreducible variety. In the case where $N$ is even, $\mathscr{O}_{\lambda}=\mathbb{O}_{\lambda} \cap G_{\text {uni }}^{\iota \theta}$ splits into two $H$-orbits since $(N)$ is an even partition. Thus if we decompose $\mathscr{O}_{\lambda}$ into two $H$ orbits, $\mathscr{O}_{\lambda}=\mathscr{O}_{\lambda}^{\prime} \cup \mathscr{O}_{\lambda}^{\prime \prime}, G_{\text {uni }}^{\iota \theta}=\overline{\mathscr{O}}_{\lambda}^{\prime} \cup \overline{\mathscr{O}}_{\lambda}^{\prime \prime}$ gives a decomposition of $G_{\text {uni }}^{\iota \theta}$ into irreducible components. Sunning up the above arguments, we have

Lemma 7.10. Let $\lambda=(N) \in \mathscr{P}_{N}$.

(i) Assume that $N$ is odd. Then $G_{\text {uni }}^{\text {i }}$ is irreducible, which is the closure of the $H$-orbit $\mathscr{O}_{\lambda}$.

(ii) Assume that $N$ is even. Then $G_{\mathrm{uni}}^{\mathrm{\theta}}$ consists of two irreducible components, which are the closures of $H$-orbits $\mathscr{O}_{\lambda}^{\prime}$ and $\mathscr{O}_{\lambda}^{\prime \prime}$. We have $\overline{\mathscr{O}}_{\lambda}^{\prime} \cap \overline{\mathscr{O}}_{\lambda}^{\prime \prime}=\overline{\mathscr{O}}_{\lambda^{\prime}}$, where $\mathscr{O}_{\lambda^{\prime}}$ is the $H$-orbit corresponding to $\lambda^{\prime}=(N-1,1) \in \mathscr{P}_{N}$, and $\overline{\mathscr{O}}_{\lambda^{\prime}}$ is irreducible. In particular, we have

$$
\overline{\mathscr{O}}_{\lambda}^{\prime}-\overline{\mathscr{O}}_{\lambda^{\prime}}=\mathscr{O}_{\lambda}^{\prime}, \quad \overline{\mathscr{O}}_{\lambda}^{\prime \prime}-\overline{\mathscr{O}}_{\lambda^{\prime}}=\mathscr{O}_{\lambda}^{\prime \prime}
$$

Proof. It is enough to show the latter statement of (ii). It is well-known that $\mathbb{O}_{\lambda^{\prime}}$ is the subregular unipotent class in $G_{\text {uni }}$ and that $G_{\text {uni }}-\mathbb{O}_{\lambda}=\overline{\mathbb{O}}_{\lambda^{\prime}}$. Since $\overline{\mathbb{O}}_{\lambda^{\prime}}=$ $\bigcup_{\mu \leq \lambda^{\prime}} \mathbb{O}_{\mu}$ (here $\mu \leq \lambda^{\prime}$ is the dominance order on $\mathscr{P}_{N}$ ), we have $G_{\text {uni }}^{\iota \theta}-\mathscr{O}_{\lambda}=\overline{\mathscr{O}}_{\lambda^{\prime}}=$ $\bigcup_{\mu \leq \lambda^{\prime}} \mathscr{O}_{\mu}$. (ii) follows from this. Note that $\mathscr{O}_{\lambda^{\prime}}$ is a single $H$-orbit since $\lambda^{\prime}$ is not an even partition, so $\overline{\mathscr{O}}_{\lambda^{\prime}}$ is irreducible.

7.11. Let $\lambda=(N) \in \mathscr{P}_{N}$. We discuss the relationship between the regular unipotent orbit $\mathscr{O}_{\lambda}$ and the varieties $X_{\text {uni }}$ appeared in 5.9. We consider the case where $P=B, L=T$, and $\Sigma=T$. Let $Y=Y_{(L, \Sigma)}$ and $X=\bar{Y}$. Then $X_{\text {uni }}$ is given by

$$
X_{\text {uni }}=\bigcup_{g \in H} g U^{\iota \theta} g^{-1},
$$

where $U$ is the unipotent radical of $B$. Under the notation in 1.7 , we define $x-1 \in \mathfrak{g}_{\text {nil }}$ by

$$
(x-1): f_{1} \mapsto f_{2} \mapsto \cdots \mapsto f_{n} \mapsto e_{0} \mapsto e_{n} \mapsto \cdots \mapsto e_{2} \mapsto e_{1} \mapsto 0
$$

in the case where $N$ is odd, and by

$$
(x-1): f_{1} \mapsto f_{2} \mapsto \cdots \mapsto f_{n} \mapsto e_{n} \mapsto \cdots \mapsto e_{2} \mapsto e_{1} \mapsto 0
$$

in the case where $N$ is even. Then $x \in U^{\iota \theta} \cap \mathscr{O}_{\lambda}$. In particular, $X_{\text {uni contains an }}$ $H$-orbit containing $x$. 
Assume that $N$ is odd. In this case $\mathscr{O}_{\lambda}$ is a single $H$-orbit, and is contained in $X_{\text {uni }}$. Since $X_{\text {uni }}$ is irreducible, $X_{\text {uni }}$ is a closed subset of $G_{\text {uni }}^{\iota \theta}$, and $\overline{\mathscr{O}}_{\lambda}=G_{\text {uni }}^{\iota \theta}$, we conclude that

$$
X_{\text {uni }}=G_{\text {uni }}^{\iota \theta} \text {. }
$$

Assume that $N$ is even. In this case, we need to consider the pairs $(B, T)$ and $\left(B_{1}, T\right)$, where $B_{1}=t_{n} B t_{n}^{-1}$ as in 1.9. Put $U_{1}=t_{n} U t_{n}^{-1}$. Then $X_{\text {uni associated }}$ to $(B, T)$ is defined as in (7.11.1), which we denote by $X_{\text {uni }}^{+}$. A similar variety is defined by replacing $U^{\iota \theta}$ by $U_{1}^{\iota \theta}$ in $(7.11 .1)$, which we denote by $X_{\text {uni }}^{-}$. Note that $X_{\text {uni }}^{-}=\zeta X_{\text {uni }}^{+} \zeta^{-1}$ for $\zeta \in G^{\theta}-H$. Let $\mathscr{O}_{\lambda}^{+}$be the $H$-orbit containing $x$. Then another $H$-orbit contained in $\mathscr{O}_{\lambda}$ is given by $\mathscr{O}_{\lambda}^{-}=\zeta \mathscr{O}_{\lambda}^{+} \zeta^{-1}$. We have $\mathscr{O}_{\lambda}^{+} \subset X_{\text {uni }}^{+}$ and $\mathscr{O}_{\lambda}^{-} \subset X_{\text {uni }}^{-}$. Note that $X_{\text {uni }}^{ \pm}$are irreducible, closed subsets of $G_{\text {uni }}^{\iota \theta}$. By Lemma 7.10 (ii), $\overline{\mathscr{O}}_{\lambda}^{+} \cup \overline{\mathscr{O}}_{\lambda}^{-}$gives a decomposition of $G_{\text {uni }}^{\iota \theta}$ into irreducible components. This implies that

$$
X_{\text {uni }}^{+}=\overline{\mathscr{O}}_{\lambda}^{+}, \quad X_{\text {uni }}^{-}=\overline{\mathscr{O}}_{\lambda}^{-},
$$

and $G_{\text {uni }}^{\iota \theta}=X_{\text {uni }}^{+} \cup X_{\text {uni }}^{-}$gives a decomposition into irreducible components.

\section{Structure of the algebra $\mathscr{A}_{\mathscr{E}}$}

8.1. We follow the notation in 6.1. Let $Q$ be as in 6.1. Here we assume that $M_{H} \simeq G L_{1} \times S O_{N-2}$. Hence $Q_{H}$ is a maximal parabolic subgroup of $H$, and $H / Q_{H}$ can be identified with the set of isotropic lines in $\mathbf{P}(V)$. Let $\mathscr{O}$ be an $H$-orbit in $G_{\text {uni }}^{\iota \theta}$ and $\mathscr{O}^{\prime}$ an $M_{H^{-o r b i t}}$ in $M_{\text {uni }}^{\iota \theta}$. We fix $u \in \mathscr{O}, v \in \mathscr{O}^{\prime}$, and consider the varieties, $Y_{u, v}, \widetilde{Y}_{u, v}$ as in 6.10 . Let $\widetilde{\mathscr{O}}^{\prime}$ be the $M^{\theta}$-orbit in $M^{\iota \theta}$ containing $\mathscr{O}^{\prime}$. Put

$$
\widetilde{Y}_{u, \mathscr{O}^{\prime}}=\left\{g \in H \mid g^{-1} u g \in \eta_{Q}^{-1}\left(\widetilde{\mathscr{O}}^{\prime}\right)\right\}
$$

Then $Q_{H}$ acts on $\widetilde{Y}_{u, \mathscr{O}^{\prime}}$ by $x: g \mapsto g x^{-1}$, and $\widetilde{Y}_{u, \mathscr{O}^{\prime}} / Q_{H}$ is a locally closed subset of $H / Q_{H}$. Let $\tau: \widetilde{Y}_{u, \mathscr{O}^{\prime}} \rightarrow \widetilde{\mathscr{O}}^{\prime}$ be the map defined by $g \mapsto \eta_{Q}\left(g^{-1} u g\right)$. Then $\tau$ is $M_{H^{-}}$ equivariant, and for any $v^{\prime} \in \widetilde{\mathscr{O}}^{\prime}, \tau^{-1}\left(v^{\prime}\right) \simeq \widetilde{Y}_{u, v}$. Hence $\operatorname{dim} \widetilde{Y}_{u, \mathscr{O}^{\prime}}=\operatorname{dim} \widetilde{Y}_{u, v}+\operatorname{dim} \mathscr{O}^{\prime}$, and we have

$$
\operatorname{dim} \widetilde{Y}_{u, \mathscr{O}^{\prime}} / Q_{H}=\operatorname{dim} \widetilde{Y}_{u, v}+\operatorname{dim} \mathscr{O}^{\prime}-\operatorname{dim} Q_{H}
$$

8.2. Let $\mathfrak{m}=\operatorname{Lie} M$, and consider the subvariety $\mathfrak{m}_{\text {nil }}^{-}$of $\mathfrak{m}$ on which $M_{H}$ acts. We denote by the same symbol $\mathscr{O}\left(\right.$ resp. $\left.\mathscr{O}^{\prime}\right)$ the $H$-orbit in $\mathfrak{g}_{\text {nil }}^{-}$(resp. $M_{H^{-}}$-orbit in $\mathfrak{m}_{\text {nil }}^{-}$) corresponding to $\mathscr{O}$ (resp. $\mathscr{O}^{\prime}$ ) as in Section 7 . In the Lie algebra setting, the map $\eta_{Q}: \mathfrak{q}^{-\theta} \rightarrow \mathfrak{m}^{-\theta}$ is defined similarly, where $\mathfrak{q}=\operatorname{Lie} Q$, and $\widetilde{Y}_{x, \mathscr{O}^{\prime}}$ is defined, for $x \in \mathscr{O} \subset \mathfrak{g}_{\text {nil }}^{-\theta}$, similarly to (8.1.1). We have $\widetilde{Y}_{u, \mathscr{O}^{\prime}}=\widetilde{Y}_{x, \mathscr{O}^{\prime}}$ for $x=u-1$. 
By Lemma 7.2 , the set of $G^{\theta}$-orbits in $G_{\text {uni }}^{\iota \theta}$ is parametrized by $\mathscr{P}_{N}, \mathscr{O}$ corresponds to $\lambda \in \mathscr{P}_{N}$ if $u \in \mathscr{O}$ has Jordan type $\lambda$ as an element in $G_{\text {uni }}$. Similarly, the set of $M^{\theta}$-orbits in $M_{\text {uni }}^{\iota \theta}$ is parametrized by $\mathscr{P}_{N-2}$.

Now assume that $\mathscr{O}$ corresponds to $\lambda \in \mathscr{P}_{N}$, and $\mathscr{O}^{\prime}$ corresponds to $\lambda^{\prime} \in \mathscr{P}_{N-2}$. Take $x \in \mathscr{O} \subset \mathfrak{g}_{\text {nil }}^{-}$. Put $W=\operatorname{Ker} x$, and let $\mathbf{P}(W)^{0}$ be the set of isotropic lines in $\mathbf{P}(W)$, namely, $\mathbf{P}(W)^{0}=\{\langle v\rangle \in \mathbf{P}(W) \mid\langle v, v\rangle=0\}$. Note that the form $\langle$,$\rangle induces$ a non-degenerate symmetric bilinear form on $\bar{V}=V_{1}^{\perp} / V_{1}$ for $V_{1} \in \mathbf{P}(W)^{0}$, and $\left.x\right|_{\bar{V}}$ is self-adjoint with respect to this form. Here $\mathfrak{g l}(\bar{V})$ is $H$-conjugate to $\mathfrak{m}$, and $\left.x\right|_{\bar{V}}$ gives an element in $\mathfrak{m}_{\text {nil }}^{-}$under this isomorphism. It is easy to see that

$$
\widetilde{Y}_{x, \mathscr{O}^{\prime}} / Q_{H} \simeq\left\{V_{1} \in \mathbf{P}(W)^{0}|x|_{V_{1}^{\perp} / V_{1}}: \text { Jordan type } \lambda^{\prime}\right\}
$$

8.3. We will see the variety on the right hand side on (8.2.1) more precisely. Write $\lambda \in \mathscr{P}_{N}$ as $\lambda=\left(a_{1}^{m_{1}}, a_{2}^{m_{2}}, \ldots, a_{h}^{m_{h}}\right)$ as in 7.6 , and put $W^{i}=\operatorname{Ker} x \cap \operatorname{Im} x^{a_{i}-1}$. We have a filtration of $W$ by subspaces

$$
W=W^{h} \supsetneq W^{h-1} \supsetneq \cdots \supsetneq W^{1} \supsetneq W^{0}=\{0\},
$$

where $\operatorname{dim} W^{i} / W^{i-1}=m_{i}$ for $i=1, \ldots, h$. For $v, v^{\prime} \in W^{i}$, put $\left(v, v^{\prime}\right)=\left\langle v, v_{1}^{\prime}\right\rangle$, where $v_{1}^{\prime} \in V$ is such that $x^{a_{i}-1} v_{1}^{\prime}=v^{\prime}$. By using a normal basis of $x \in \mathfrak{g}_{\text {nil }}^{-}$, one can check that this gives a well-defined symmetric bilinear form on $W^{i}$, which is identically zero on $W^{i-1}$. The induced form $($,$) on W^{i} / W^{i-1}$ gives a non-degenerate symmetric bilinear form on $W^{i} / W^{i-1}$. For each non-zero $v \in W^{i}$, let $V_{1}=\langle v\rangle$ be the line spanned by $v$. Since $v \in \operatorname{Ker} x, x$ induces a linear map $\bar{x}=\left.x\right|_{\bar{V}}$ on $\bar{V}=V_{1}^{\perp} / V_{1}$. We have $\bar{x} \in \mathfrak{g l}(\bar{V})_{\text {nil }}^{-} \simeq \mathfrak{m}_{\text {nil }}^{-}$. Define a variety $S_{i}$ of $V$ such that $W^{i-1} \subset S_{i} \subset W^{i}$ by

$$
S_{i}=\left\{v \in W^{i} \mid(v, v)=0\right\} .
$$

We consider the following conditions on $\lambda \in \mathscr{P}_{N}$ and $\lambda^{\prime} \in \mathscr{P}_{N-2}$.

$\left(\mathrm{A}_{i}\right)$ The Young diagram of $\lambda^{\prime}$ is obtained from that of $\lambda$ by replacing one row of length $a_{i}$ by a row of length $a_{i}-2$.

$\left(\mathrm{B}_{i}\right)$ The Young diagram of $\lambda^{\prime}$ is obtained from that of $\lambda$ by replacing two rows of length $a_{i}$ by two rows of length $a_{i}-1$ ( in this case, we assume that $m_{i} \geq 2$ ).

Moreover, we divide $\left(\mathrm{A}_{i}\right)$ into two cases $\left(\mathrm{A}_{i}^{\prime}\right)$ and $\left(\mathrm{A}_{i}^{\prime \prime}\right)$. Here $\left(\mathrm{A}_{i}^{\prime}\right)$ is the case where $a_{i+1} \leq a_{i}-2$, and $\left(\mathrm{A}_{i}^{\prime \prime}\right)$ is the case where $a_{i+1}=a_{i}-1$.

The following lemma can be checked easily, by using a normal basis of $x$. (It is reduced to the case where $\lambda=\left(a^{m}\right)$ with $m=1$ or 2$)$.

Lemma 8.4. Under the notation above, let $\lambda^{\prime}$ be the Jordan type of $\bar{x} \in \mathfrak{g l}(\bar{V})_{\text {nil }}^{-}$. Then we have

(i) If $v \notin S_{i}, \lambda^{\prime}$ is obtained from $\lambda$ by the procedure $\left(\mathrm{A}_{i}\right)$.

(ii) If $v \in S_{i}$ (in this case $m_{i} \geq 2$ ), $\lambda^{\prime}$ is obtained from $\lambda$ by the procedure $\left(\mathrm{B}_{i}\right)$.

The following corollary is immediate from Lemma 8.4. 
Corollary 8.5. $\widetilde{Y}_{x, \mathscr{O}^{\prime}}=\emptyset$ unless $\lambda^{\prime}$ is obtained from $\lambda$ by $\left(\mathrm{A}_{i}\right),\left(\mathrm{B}_{i}\right)$ for some $i$. If $\widetilde{Y}_{x, O^{\prime}} \neq \emptyset$, then

$$
\operatorname{dim} \tilde{Y}_{x, \mathscr{O}^{\prime}} / Q_{H}= \begin{cases}m_{1}+\cdots+m_{i}-1, & \text { case }\left(\mathrm{A}_{i}\right), \\ m_{1}+\cdots+m_{i}-2, & \text { case }\left(\mathrm{B}_{i}\right) .\end{cases}
$$

8.6. Recall that $\operatorname{dim} Y_{u, v} \leq s$, where $s=\left(\operatorname{dim} Z_{H}(u)-\operatorname{dim} Z_{M_{H}}(v)\right) / 2+\Delta_{Q} / 2$ by 6.10 . Since we know by Lemma 7.4 that $\operatorname{dim} Z_{H}(u)=n(\lambda)$ if $u \in G_{\text {uni }}^{\iota \theta}$ has Jordan type $\lambda$, the number $s$ can be computed explicitly. We have the following.

Proposition 8.7. Assume that $u \in \mathscr{O}$ has type $\lambda, v \in \mathscr{O}^{\prime}$ has type $\lambda^{\prime}$. Then $Y_{u, v}$ is non-empty if and only if $\lambda^{\prime}$ is obtained from $\lambda$ by the procedure $\left(\mathrm{A}_{i}\right)$, or $\left(\mathrm{B}_{i}\right)$. We have $\operatorname{dim} Y_{u, v}=s$ if and only if $\lambda^{\prime}$ is obtained from $\lambda$ by $\left(\mathrm{A}_{i}^{\prime}\right)$.

Proof. Since $Y_{u, v} \neq \emptyset$ if and only if $\widetilde{Y}_{x, \mathscr{O}^{\prime}} \neq \emptyset$, the first assertion follows from Corollary 8.5. Now assume $Y_{u, v} \neq \emptyset$. Since $\operatorname{dim} Y_{u, v}=\operatorname{dim} \widetilde{Y}_{x, \mathscr{O}^{\prime}} / Q_{H}, \operatorname{dim} Y_{u, v}$ can be computed from the formula in Corollary 8.5. Since $M_{H} \simeq G L_{1} \times S O_{N-2}, \operatorname{dim} Z_{M_{H}}(v)=$ $n\left(\lambda^{\prime}\right)+1$ by Lemma 7.4. Then we have

$$
\operatorname{dim} Z_{H}(u)-\operatorname{dim} Z_{M_{H}}(v)= \begin{cases}2\left(m_{1}+\cdots+m_{i}-1\right)-1 & \text { case }\left(\mathrm{A}_{i}^{\prime}\right) \\ 2\left(m_{1}+\cdots+m_{i}-1\right) & \text { case }\left(\mathrm{A}_{i}^{\prime \prime}\right) \\ 2\left(m_{1}+\cdots+m_{i}-2\right) & \text { case }\left(\mathrm{B}_{i}\right)\end{cases}
$$

Since $\Delta_{Q}=1$, we have

$$
s= \begin{cases}m_{1}+\cdots+m_{i}-1 & \text { case }\left(\mathrm{A}_{i}^{\prime}\right) \\ \left(m_{1}+\cdots+m_{i}-1\right)+1 / 2 & \text { case }\left(\mathrm{A}_{i}^{\prime \prime}\right) \\ \left(m_{1}+\cdots+m_{i}-2\right)+1 / 2 & \text { case }\left(\mathrm{B}_{i}\right)\end{cases}
$$

The proposition follows.

8.8. For $x \in \mathfrak{g}_{\text {nil }}^{-}$, let $\mathscr{B}_{x}=\left\{g B_{H} \in H / B_{H} \mid g^{-1} x \in\right.$ Lie $\left.B_{H}\right\}$ be the Springer fibre of $x$. We also define $\mathscr{P}_{x}=\left\{g Q_{H} \in H / Q_{H} \mid g^{-1} x \in\right.$ Lie $\left.Q_{H}\right\}$ and a natural map $\pi_{Q}: \mathscr{B}_{x} \rightarrow \mathscr{P}_{x}, g B_{H} \mapsto g Q_{H}$. $\mathscr{P}_{x}$ can be identified with $\mathbf{P}(W)^{0}$. Thus $\mathscr{P}_{x}$ is partitioned into locally closed pieces $\mathscr{P}_{x, \lambda^{\prime}} \simeq \widetilde{Y}_{x, \mathscr{O}^{\prime}} / Q_{H}$, where $\widetilde{\mathscr{O}}^{\prime}$ is the $M^{\theta}$-orbit corresponding to $\lambda^{\prime} \in \mathscr{P}_{N-2}$, and we have a partition of $\mathscr{B}_{x}$ into locally closed pieces

$$
\mathscr{B}_{x}=\coprod_{\lambda^{\prime} \in \mathscr{P}_{N-2}} \pi_{Q}^{-1}\left(\mathscr{P}_{x, \lambda^{\prime}}\right)
$$

Assume that $x \in \mathscr{O}$ has type $\lambda$. By Lemma 8.4, if $\pi_{Q}^{-1}\left(\mathscr{P}_{x, \lambda^{\prime}}\right) \neq \emptyset$, then $\lambda^{\prime}$ is obtained from $\lambda$ by the procedure given in (i) or (ii) in Lemma 8.4. If $v \in W$ is given, $\bar{x} \in \mathfrak{g l}(\bar{V})_{\text {nil }}^{-}$is defined as above. We denote by $\overline{\mathscr{B}}_{\bar{x}}$ the corresponding Springer fibre for $S O(\bar{V})$. By applying Proposition 2.2 (ii) for the case $P=B, L=T$, we have 


$$
\begin{aligned}
\operatorname{dim} \mathscr{B}_{x} & \leq \nu_{H}-\operatorname{dim} \mathscr{O} / 2+\Delta_{B} / 2 \\
& =\operatorname{dim} Z_{H}(x) / 2
\end{aligned}
$$

where $\Delta_{B}=[N / 2]=\operatorname{dim} T_{H}$.

The following result was proved by [CVX1, Proposition 3.1] in the case where $N$ is odd, and $\mathbf{k}=\mathbf{C}$. The discussion below seems to be simpler. (But this result will not be used in later discussions.)

Proposition 8.9. Assume that $x \in \mathscr{O}$ has type $\lambda$. Then $\operatorname{dim} \mathscr{B}_{x}=\operatorname{dim} Z_{H}(x) / 2$ if and only if $\lambda=\left(\lambda_{1}, \ldots, \lambda_{r}\right) \in \mathscr{P}_{N}$ satisfies the condition that $\lambda_{i}$ is even for $i \geq 2$ (hence $\lambda_{1}$ is odd (resp. even) if $N$ is odd (resp. even)).

Proof. In the notation of 8.8, the restriction of the map $\pi_{Q}: \mathscr{B}_{x} \rightarrow \mathscr{P}_{x}$ on $\pi_{Q}^{-1}\left(\mathscr{P}_{x, \lambda^{\prime}}\right)$ is surjective, and each fibre is isomorphic to $\overline{\mathscr{B}}_{\bar{x}}$. Hence

$$
\operatorname{dim} \pi_{Q}^{-1}\left(\mathscr{P}_{x, \lambda^{\prime}}\right)=\operatorname{dim} \mathscr{P}_{x, \lambda^{\prime}}+\operatorname{dim} \overline{\mathscr{B}}_{\bar{x}},
$$

and $\operatorname{dim} \mathscr{B}_{x}=\max \left\{\operatorname{dim} \pi_{Q}^{-1}\left(\mathscr{P}_{x, \lambda^{\prime}}\right) \mid \lambda^{\prime} \in \mathscr{P}_{N-2}\right\} . \operatorname{dim} \mathscr{P}_{x, \lambda^{\prime}}$ is given in Corollary 8.5. If we denote $\bar{H}=S O(\bar{V})$, then $\operatorname{dim} \overline{\mathscr{B}}_{\bar{x}} \leq \operatorname{dim} Z_{\bar{H}}(\bar{x}) / 2$, and $\operatorname{dim} Z_{\bar{H}}(\bar{x})=n\left(\lambda^{\prime}\right)$. Thus by using a similar argument as in the proof of Proposition 8.7, we see that $\operatorname{dim} \mathscr{B}_{x}=\operatorname{dim} Z_{H}(x) / 2$ if and only if there exists $i$ such that $\lambda^{\prime} \in \mathscr{P}_{N-2}$ is obtained from $\lambda$ by $\left(\mathrm{A}_{i}^{\prime}\right)$ and that $\operatorname{dim} \overline{\mathscr{B}}_{\bar{x}}=\operatorname{dim} Z_{\bar{H}}(\bar{x}) / 2$. The proposition follows from this by induction on $N$.

8.10. $Z_{H}(x)$ acts on $\widetilde{Y}_{x, \mathscr{O}^{\prime}}$ by $h: g \mapsto h g$, hence induces an action of $Z_{H}(x)$ on $\widetilde{Y}_{x, \mathscr{O}^{\prime}} / Q_{H}$. Put $C_{i}=W^{i}-S_{i}$, and let $p\left(C_{i}\right), p\left(S_{i}^{*}\right)$ be the image of $C_{i}, S_{i}^{*}=S_{i}-\{0\}$ under the map $p: W^{i}-\{0\} \rightarrow \mathbf{P}(W)$. Then by Lemma 8.4, the variety $\widetilde{Y}_{x, \mathscr{O}^{\prime}} / Q_{H}$ is isomorphic to $p\left(C_{i}\right)$ or $p\left(S_{i}^{*}\right)$ if $\mathscr{O}^{\prime}$ corresponds to $\lambda^{\prime}$, which is obtained from $\lambda$ as in Lemma 8.4 for some $i$. The corresponding action of $Z_{H}(x)$ on $p\left(C_{i}\right)$ or $p\left(S_{i}^{*}\right)$ is given as follows; The natural action of $Z_{H}(x)$ on $V$ leaves $W^{i}$ invariant. Moreover, the bilinear form (, ) on $W^{i}$ is $Z_{H}(x)$-invariant. Hence $S_{i}$ and $C_{i}$ are stable by the action of $Z_{H}(x)$. The induced action on $p\left(S_{i}^{*}\right)$ or $p\left(C_{i}\right)$ coincides with the action of $Z_{H}(x)$ on $\widetilde{Y}_{x, O^{\prime}} / Q_{H}$. We show the following lemma.

Lemma 8.11. (i) The action of $Z_{H}(u)$ on $\widetilde{Y}_{u, \sigma^{\prime}} / Q_{H}$ is transitive.

(ii) The action of $Z_{H}(u) \times Z_{M_{H}}(v)$ on $Y_{u, v}$ is transitive.

Proof. (i) It is enough to show that $Z_{H}(x)$ acts transitively on $p\left(C_{i}\right)$ or $p\left(S_{i}^{*}\right)$. Here we only show the case of $p\left(C_{i}\right)$, since this is the case needed in later discussions. The case of $p\left(S_{i}^{*}\right)$ is proved similarly. Put $a=\operatorname{dim} W^{i}, b=m_{i}=\operatorname{dim} W^{i} / W^{i-1}$ and $r=[b / 2]$. Take $v \in C_{i}$. Then there exists a basis $\left\{v_{1}, \cdots, v_{a}\right\}$ of $W^{i}$ such that $\left\{v_{b+1}, \cdots, v_{a}\right\}$ gives a basis of $W^{i-1}$, satisfying the condition that $v=v_{1}+v_{b}$ and that, for any $w=\sum_{j} x_{j} v_{j} \in W^{i}$,

$$
(w, w)=x_{1} x_{b}+x_{2} x_{b-1}+\cdots+x_{r} x_{b-r}+\delta x_{r+1}^{2},
$$


where $\delta=1$ if $b$ is odd and $\delta=0$ if $b$ is even. Let $C_{i}^{0}$ be the set of $w=\sum_{j} x_{j} v_{j} \in C_{i}$ such that $x_{1} \neq 0$. Thus $C_{i}^{0}$ is an open dense subset of $C_{i}$ containing $v$. We show

(8.11.1) For any $w \in C_{i}^{0}$, there exists $\phi_{w} \in Z_{H}(x)$ such that $\phi_{w}(v)=\gamma^{-1} w$, where we put $(w, w)=\gamma^{2} \in \mathbf{k}^{*}$.

In fact, for a given $w \in C_{i}^{0}$, we choose $\gamma$ such that $(w, w)=\gamma^{2}$, and define a $\operatorname{map} \phi_{w}^{\prime}: W^{i} \rightarrow W^{i}$ by

$$
\left\{\begin{array}{l}
v \mapsto \gamma^{-1} w, \\
v_{b} \mapsto x_{1}^{-1} \gamma v_{b}, \\
v_{j} \mapsto v_{j}-x_{b-j+1} v_{b} \quad \text { if } 1<j<b, \\
v_{j} \mapsto v_{j} \quad \text { if } j>b .
\end{array}\right.
$$

It is easy to check that $\phi_{w}^{\prime}$ preserves the symmetric bilinear form $($,$) on W^{i}$, and the restriction of $\phi_{w}^{\prime}$ on $W^{i+1}$ is identity. Moreover $\operatorname{det} \phi_{w}^{\prime}=1$. For $\lambda=\left(\lambda_{1}, \ldots, \lambda_{r}\right)$, let $\left\{w_{i, j}\right\}$ be a normal basis of $x$. For any $i$, we define $V^{i}$ as the subspace of $V$ spanned by $\left\{w_{k, j} \mid 1 \leq k \leq i\right\}$. Then $V^{i}$ is an $x$-stable subspace of $V$ such that $V^{i} \cap \operatorname{Ker} x=W^{i}$. The linear map $\phi_{w}^{\prime}$ on $W^{i}$ can be extended in a canonical way to a linear automorphism $\phi_{w}^{\prime \prime}$ on $V^{i}$ which commutes with $x$, and preserves the form $\langle$,$\rangle . We extend \phi_{w}^{\prime \prime}$ to a linear map $\phi_{w}$ on $V$ by defining $w_{k, j} \mapsto w_{k, j}$ for any $k>a$. Then $\phi_{w} \in Z_{H}(x)$, and satisfies the condition in (8.11.1). Thus (8.11.1) was proved.

Now (8.11.1) shows that the action of $Z_{H}(x)$ on $p\left(C_{i}\right)$ is transitive on $p\left(C_{i}^{0}\right)$. For any $v \in C_{i}$, we can find such $C_{i}^{0}$ containing $v$. Hence such $p\left(C_{i}^{0}\right)$ covers whole $p\left(C_{i}\right)$. Since $p\left(C_{i}^{0}\right)$ is open dense in $p\left(C_{i}\right)$, we conclude that $Z_{H}(x)$ acts transitively on $p\left(C_{i}\right)$. Thus (i) is proved.

(ii) It follows from (i) that the action of $Z_{H}(u)$ on $Y_{u, v}$ is transitive modulo $Q_{H}$, namely, for any $g Z_{M_{H}}(v)^{0} U_{Q}^{\theta} \in Y_{u, v}$, there exists $z \in Z_{H}(u)$ and $q \in Q_{H}$ such that $z g q Z_{M_{H}}(v)^{0} U_{Q}^{\theta}=Z_{M_{H}}(v)^{0} U_{Q}^{\theta}$. Here we may replace $q$ by $m \in M_{H}$. Then the definition of $Y_{u, v}$ implies that $m^{-1}\left(g^{-1} u g\right) m \in \eta_{Q}^{-1}(v)$. But this implies that $m \in M_{H}(v)$. Hence $Z_{H}(u) \times Z_{M_{H}}(v)$ acts transitively on $Y_{u, v}$. (ii) is proved.

8.12. Take $x \in \mathscr{O} \subset \mathfrak{g}_{\text {nil }}^{-}$, where the Jordan type of $x$ is $\lambda$. The structure of the group $A_{H}(x)$ is described in 7.7. Now assume that $x \in \mathfrak{q}_{\text {nil }}$ and let $\bar{x} \in \mathfrak{m}_{\text {nil }}^{-}$ be the image of $x$ under the map $\eta_{Q}: \mathfrak{q} \rightarrow \mathfrak{m}$. Assume that the Jordan type of $\bar{x}$ is $\lambda^{\prime} . A_{M_{H}}(\bar{x})$ is described similarly. Put $Z_{Q_{H}}(x)=Z_{H}(x) \cap Q_{H}$, and let $A_{Q_{H}}(x)$ be the image of $Z_{Q_{H}}(x)$ under the natural map $Z_{H}(x) \rightarrow A_{H}(x)$. The projection $Q_{H} \rightarrow M_{H}$ induces a map $Z_{Q_{H}}(x) \rightarrow Z_{M_{H}}(\bar{x})$, which gives a natural homomorphism $\varphi: A_{Q_{H}}(x) \rightarrow A_{M_{H}}(\bar{x})$.

Now assume that $\lambda^{\prime}$ is obtained from $\lambda$ by $\left(\mathrm{A}_{i}^{\prime}\right)$. In this case, one can check that $A_{Q_{H}}(x)=A_{H}(x)$. Hence $\varphi$ gives rise to a map $\varphi: A_{H}(x) \rightarrow A_{M_{H}}(\bar{x})$. The image $\operatorname{Im} \varphi \subset A_{M_{H}}(\bar{x})$ is described as follows; we consider two cases, according to the case where $a_{i+1}=a_{i}-2$ or $a_{i+1}<a_{i}-2$ (note that $a_{i+1} \leq a_{i}-2$ by the assumption $\left(\mathrm{A}_{i}^{\prime}\right)$.)

Case I. $a_{i+1}=a_{i}-2$. In this case, $\lambda^{\prime}=\left(a_{1}^{m_{1}}, \ldots, a_{i}^{m_{i}-1}, a_{i+1}^{m_{i+1}+1}, \ldots, a_{h}^{m_{h}}\right)$. Then $A_{M_{H}}(\bar{x})=\left\{\alpha^{\prime}=\left(\alpha_{1}^{\prime}, \ldots \alpha_{i}^{\prime}, \alpha_{i+1}^{\prime}, \ldots, \alpha_{h}^{\prime}\right)\right\}$ (here we ignore $\alpha_{i}^{\prime}$ if $m_{i}=1$ ). $\varphi$ is given 
by $\alpha_{j} \mapsto \alpha_{j}^{\prime}$ for $j \neq i$, and $\alpha_{i} \mapsto \alpha_{i+1}^{\prime}$. We have $\operatorname{Im} \varphi=\left\{\alpha^{\prime} \in A_{M_{H}}(\bar{x}) \mid \alpha_{i}^{\prime}=1\right\}$, which is an index two subgroup of $A_{M_{H}}(\bar{x})$ if $m_{i} \geq 2$, and $\varphi$ is surjective if $m_{i}=1$.

Case II. $a_{i+1}<a_{i}-2$. In this case, $\lambda^{\prime}=\left(a_{1}^{m_{1}}, \ldots, a_{i}^{m_{i}-1}, a_{i}-2, a_{i+1}^{m_{i+1}}, \ldots, a_{h}^{m_{h}}\right)$. Then $A_{M_{H}}(\bar{x})=\left\{\alpha^{\prime}=\left(\alpha_{1}^{\prime}, \ldots, \alpha_{i}^{\prime}, \alpha_{*}^{\prime}, \alpha_{i+1}^{\prime}, \ldots, \alpha_{h}^{\prime}\right)\right\}$. The map $\varphi$ is given by $\alpha_{j} \mapsto \alpha_{j}^{\prime}$ if $j \neq i$, and $\alpha_{i} \mapsto \alpha_{*}^{\prime}$. We have $\operatorname{Im} \varphi=\left\{\alpha^{\prime} \in A_{M_{H}}(\bar{x}) \mid \alpha_{i}^{\prime}=1\right\}$, which is an index 2 subgroup of $A_{M_{H}}(\bar{x})$ if $m_{i} \geq 2$, and $\varphi$ is an isomorphism if $m_{i}=1$.

8.13. We now consider the variety $Y_{u, v}=Y_{x, \bar{x}}$ under the assumption that $\lambda^{\prime}$ is obtained from $\lambda$ by $\left(\mathrm{A}_{i}^{\prime}\right)$. Recall that $I\left(Y_{x, \bar{x}}\right)$ is the set of irreducible components of $Y_{x, \bar{x}}$ of dimension s. By Proposition 8.7, we have $\operatorname{dim} Y_{x, \bar{x}}=s$, hence $I\left(Y_{x, \bar{x}}\right) \neq \emptyset$. By Lemma 8.11, $Z_{H}(x) \times Z_{M_{H}}(\bar{x})$ acts transitively on $Y_{x, \bar{x}}$, hence $A_{H}(x) \times A_{M_{H}}(\bar{x})$ acts transitively on $I\left(Y_{x, \bar{x}}\right)$. In order to determine the permutation representation $\varepsilon_{x, \bar{x}}$ of $A_{H}(x) \times A_{M_{H}}(\bar{x})$ on $I\left(Y_{x, \bar{x}}\right)$, it is enough to determine an isotropy subgroup of $A_{H}(x) \times A_{M_{H}}(\bar{x})$. By the definition of $Y_{x, \bar{x}}$, we can write as

$$
Y_{x, \bar{x}}=\bigcup_{\alpha \in A_{H}(x), \alpha^{\prime} \in A_{M_{H}}(\bar{x})} \alpha Z_{H}^{0}(x) U_{Q}^{\theta} Z_{M_{H}}^{0}(\bar{x}) \alpha^{\prime} .
$$

Hence $Z_{H}^{0}(x) U_{Q}^{\theta} Z_{M_{H}}^{0}(\bar{x})$ is an irreducible component in $Y_{x, \bar{x}}$. We denote by $E$ the stabilizer of the component $Z_{H}(x)^{0} U_{Q}^{\theta} Z_{M_{H}}(\bar{x})^{0}$ in $A_{H}(x) \times A_{M_{H}}(\bar{x})$. We have the following lemma.

Lemma 8.14. Under the notation as above, we have

$$
E= \begin{cases}\left\{\left(\alpha, \alpha^{\prime}\right) \in A_{H}(x) \times A_{M_{H}}(\bar{x}) \mid \alpha_{j}=\alpha_{j}^{\prime}(j \neq i), \alpha_{i}=\alpha_{i+1}^{\prime}\right\}, & \text { case (I), } \\ \left\{\left(\alpha, \alpha^{\prime}\right) \in A_{H}(x) \times A_{M_{H}}(\bar{x}) \mid \alpha_{j}=\alpha_{j}^{\prime}(j \neq i), \alpha_{i}=\alpha_{*}^{\prime}\right\}, & \text { case (II). }\end{cases}
$$

Proof. Since $A_{H}(x)=A_{Q_{H}}(x)$, we have, for $\left(\alpha, \alpha^{\prime}\right) \in A_{H}(x) \times A_{M_{H}}(\bar{x})$,

$$
\alpha A_{H}^{0}(x) U_{Q}^{\theta} A_{M_{H}}^{0}(\bar{x}) \alpha^{\prime}=A_{H}^{0}(x) U_{Q}^{\theta} A_{M_{H}}^{0}(\bar{x}) \varphi(\alpha) \alpha^{\prime} .
$$

Hence the lemma follows from the discussion in 8.12.

8.15. We denote an element $\tau$ of $A_{G^{\theta}}(x)^{\wedge} \simeq(\mathbf{Z} / 2 \mathbf{Z})^{h}$ as $\tau=\left(\tau_{1}, \ldots, \tau_{h}\right) \in$ $(\mathbf{Z} / 2 \mathbf{Z})^{h}$, with $\tau_{i}= \pm 1$. Let $\tau_{i}=(-1)_{i}$ be the character of $A_{G^{\theta}}(x)$ corresponding to the generator of the $i$-th component $\mathbf{Z} / 2 \mathbf{Z}$. Then $A_{H}(x)^{\wedge}$ is given as

$$
A_{H}(x)^{\wedge}=A_{G^{\theta}}(x)^{\wedge} / \prod_{a_{i}: \text { odd }}(-1)_{i},
$$

hence, we may identify $A_{H}(x)^{\wedge}$ with the following subset of $A_{G^{\theta}}(x)^{\wedge}$,

$$
A_{H}(x)^{\wedge}=\left\{\tau=\left(\tau_{1}, \ldots, \tau_{h}\right) \in(\mathbf{Z} / 2 \mathbf{Z})^{h} \mid \tau_{i_{0}}=1\right\},
$$

where $i_{0}$ is the index such that $a_{i_{0}}$ is the largest odd number among $a_{1}, \ldots, a_{h}$. 
Let $\lambda, \lambda^{\prime}$ be as in 8.12, and we return to the setting that $u \in \mathscr{O} \subset G_{\text {uni }}^{\iota \theta}, v \in$ $\mathscr{O}^{\prime} \subset M_{\text {uni }}^{\iota \theta}$. Then we can write $\tau \in A_{H}(u)^{\wedge}$ as $\tau=\left(\tau_{1}, \ldots, \tau_{h}\right)$. As in 8.12, according to the case (I) or (II), we can write $\tau^{\prime} \in A_{M_{H}}(v)^{\wedge}$ as $\tau^{\prime}=\left(\tau_{1}^{\prime}, \ldots, \tau_{i}^{\prime}, \tau_{i+1}^{\prime}, \ldots, \tau_{h}^{\prime}\right)$ or $\tau^{\prime}=\left(\tau_{1}^{\prime}, \ldots, \tau_{i}^{\prime}, \tau_{*}^{\prime}, \tau_{i+1}^{\prime}, \ldots, \tau_{h}^{\prime}\right)$. Note that this notation is compatible with the identification in (8.15.1). We define a subset $D$ of $A_{H}(u)^{\wedge} \times A_{M_{H}}(v)^{\wedge}$ by

$$
D= \begin{cases}\left\{\left(\tau, \tau^{\prime}\right) \in A_{H}(u)^{\wedge} \times A_{M_{H}}(v)^{\wedge} \mid \tau_{j}=\tau_{j}^{\prime}(j \neq i), \tau_{i}=\tau_{i+1}^{\prime}\right\}, & \text { case (I), } \\ \left\{\left(\tau, \tau^{\prime}\right) \in A_{H}(u)^{\wedge} \times A_{M_{H}}(v)^{\wedge} \mid \tau_{j}=\tau_{j}^{\prime}(j \neq i), \tau_{i}=\tau_{*}^{\prime}\right\}, & \text { case (II) } .\end{cases}
$$

Let $\varepsilon_{u, v}$ be the permutation representation of $A_{H}(u) \times A_{M_{H}}(v)$ on $I\left(Y_{u, v}\right)$ as before. It follows from the previous discussions, we have

Proposition 8.16. $A_{H}(u) \times A_{M_{H}}(v)$-module $\varepsilon_{u, v}$ can be decomposed into irreducible modules as

$$
\varepsilon_{u, v} \simeq \bigoplus_{\left(\tau, \tau^{\prime}\right) \in D} \tau \otimes \tau^{\prime}
$$

8.17. We generalize the notation of $\tau \in A_{H}(u)^{\wedge}$ as follows; let $u \in \mathscr{O}$ with type $\lambda$. Write $\lambda \in \mathscr{P}_{N}$ as $\lambda=\left(\lambda_{1}, \ldots, \lambda_{N}\right)$ with $\lambda_{1} \geq \lambda_{2} \geq \cdots \geq \lambda_{N} \geq 0$. Correspondingly, we consider the symbol $\tau=\left(\tau_{1}, \ldots, \tau_{N}\right)$ satisfying the following properties,

(i) $\tau_{i}= \pm 1$ for any $i$, and $\tau_{i}=1$ if $\lambda_{i}=0$,

(ii) $\tau_{i}=\tau_{j}$ if $\lambda_{i}=\lambda_{j}$,

(iii) $\tau_{i}=1$ for $i$ such that $\lambda_{i}$ is the largest odd number among $\lambda_{1}, \ldots, \lambda_{N}$.

The set of such symbols $\tau$ is in bijection with $A_{H}(u)^{\wedge}$. If $\mathscr{E}$ is a local system on $\mathscr{O}$ corresponding to $\tau \in A_{H}(u)^{\wedge}$, we denote it by $\mathscr{E}=\mathscr{E}_{\tau}$.

8.18. Take $\left(L \subset P, \mathscr{O}_{L}, \mathscr{E}_{1}^{\dagger}\right) \in \mathscr{S}_{G}$. We assume that $L_{H} \simeq\left(G L_{1}\right)^{a} \times S O_{N_{0}}$ with $N_{0}+2 a=N$. Assume that the Jordan type of $\mathscr{O}_{L}$ is $\nu=\left(\nu_{1}, \ldots, \nu_{N_{0}}\right)$, and $\mathscr{E}_{1}^{\dagger}=\mathscr{E}_{\sigma}^{\dagger}$ with $\sigma=\left(\sigma_{1}, \ldots, \sigma_{N_{0}}\right)$. For $i=0,1, \ldots, a$, let $P^{(i)}$ be the $\theta$-stable parabolic subgroup of $G$ containing $P$, and $L^{(i)}$ the $\theta$-stable Levi subgroup of $P^{(i)}$ containing $L$ such that $L_{H}^{(i)} \simeq\left(G L_{1}\right)^{a-i} \times S O_{N_{0}+2 i}$. Hence $P_{H}^{(a-1)}=Q_{H}$ is the maximal parabolic subgroup of $H$, and $P_{H}^{(0)}=P_{H}$. We consider $\pi: \widetilde{X} \rightarrow X$ with respect to $\left(L \subset P, \mathscr{O}_{L}, \mathscr{E}_{1}^{\dagger}\right)$ and consider the semisimple perverse sheaf $K=\pi_{*} K_{\overline{\mathscr{E}}_{1}}$ on $X$. We can define a similar complex $K^{(i)}$ on $X^{(i)} \subset\left(L^{(i)}\right)^{\iota \theta}$, by replacing $G$ by $L^{(i)}$. The following lemma is a generalization of Proposition 8.9.

Lemma 8.19. Let $(\mathscr{O}, \mathscr{E}) \in \mathscr{N}_{G}$, and assume that $(\mathscr{O}, \mathscr{E})$ belongs to $\left(L \subset P, \mathscr{O}_{L}, \mathscr{E}_{1}^{\dagger}\right)$. Then the Jordan type $\lambda=\left(\lambda_{1}, \ldots, \lambda_{N}\right)$ of $\mathscr{O}$ satisfies the condition

$$
\lambda_{i}-\nu_{i} \in 2 \mathbf{Z}_{\geq 0} \quad \text { for each } i .
$$

(Here we write $\nu=\left(\nu_{1}, \ldots, \nu_{N}\right)$ by putting $\nu_{i}=0$ for $i>N_{0}$.) 
Proof. We can formulate a similar property as in the lemma by replacing $G$ by $L^{(i)}$. We shall prove the claim of the lemma by induction on $i$. So we may assume that the claim holds for $i=a-1$, i.e., in the case where $P_{H}^{(i)}$ is the maximal parabolic subgroup $P_{H}^{(a-1)}=Q_{H}$. Let $\rho \in \mathscr{A}_{\mathscr{E} 1}^{\wedge}$ be the irreducible character corresponding to $(\mathscr{O}, \mathscr{E})$ under the generalized Springer correspondence. Let $\mathscr{A}_{\mathscr{E}_{1}}^{\prime}$ be the subalgebra of $\mathscr{A}_{\mathscr{E}_{1}}$ associated to $Q_{H}$, and take $\rho^{\prime} \in\left(\mathscr{A}_{\mathscr{E} 1}^{\prime}\right)^{\wedge}$ such that $\left\langle\rho, \rho^{\prime}\right\rangle \neq 0$. Let $\left(\mathscr{O}^{\prime}, \mathscr{E}^{\prime}\right) \in \mathscr{N}_{M}$ be the pair corresponding to $\rho^{\prime}$ under the generalized Springer correspondence. Take $u \in \mathscr{O}, v \in \mathscr{O}^{\prime}$, and write $\mathscr{E}=\mathscr{E}_{\tau}, \mathscr{E}^{\prime}=\mathscr{E}_{\tau^{\prime}}^{\prime}$ for $\tau \in A_{H}(u)^{\wedge}, \tau^{\prime} \in A_{M_{H}}(v)^{\wedge}$. Then by Corollary 6.11, $\tau \otimes \tau^{\prime}$ appears in the decomposition of $\varepsilon_{u, v}$. In particular, $I\left(Y_{u, v}\right)$ is non-empty. Hence by Proposition 8.7, the Jordan type $\lambda^{\prime}$ of $\mathscr{O}^{\prime}$ is obtained from $\lambda$ by the procedure $\left(A_{i}^{\prime}\right)$ for some $i$. By induction hypothesis, $\left(\mathscr{O}^{\prime}, \mathscr{E}^{\prime}\right)$ satisfies the claim of the lemma. Hence $\lambda$ also satisfies the claim. The lemma is proved.

Proposition 8.20. Let $\mathscr{O}_{0}$ be a unique $H$-orbit in $X_{\text {uni }}$ such that $\mathscr{O}_{0} \cap \eta_{P}^{-1}\left(\mathscr{O}_{L}\right)$ is open dense in $\eta_{P}^{-1}\left(\mathscr{O}_{L}\right)$.

(i) $\mathscr{O}_{0}$ is the unique open dense orbit contained in $X_{\text {uni. }}$.

(ii) The Jordan type of $\mathscr{O}_{0}$ is given by $\lambda=\left(2 a+\nu_{1}, \nu_{2}, \ldots, \nu_{N}\right)$.

Proof. (i) Put $\mathscr{O}=\mathscr{O}_{0}$. By the assumption, $\overline{\eta_{P}^{-1}\left(\mathscr{O}_{L}\right)} \subset \overline{\mathscr{O}}$. Since $X_{\text {uni }}$ is a union of $H$-conjugates of $\eta_{P}^{-1}\left(\overline{\mathscr{O}}_{L}\right), X_{\text {uni }} \subset \overline{\mathscr{O}}$. As $\mathscr{O} \subset X_{\text {uni }}$, we have $\overline{\mathscr{O}}=X_{\text {uni }}$. Since $X_{\text {uni }}$ is irreducible, $\mathscr{O}$ is uniquely determined.

(ii) By Lemma 5.10, $\operatorname{dim} X_{\text {uni }}=2 \nu_{H}-2 \nu_{L_{H}}+\operatorname{dim} \mathscr{O}_{L}+a\left(\right.$ here $\left.a=\Delta_{P}\right)$. Hence $\operatorname{dim} \mathscr{O}=\operatorname{dim} H-\operatorname{dim} L_{H}+\operatorname{dim} \mathscr{O}_{L}+a$. But $\operatorname{dim} L_{H}-\operatorname{dim} \mathscr{O}_{L}-a=\operatorname{dim} Z_{\bar{H}}(v)$ for $v \in \mathscr{O}_{L}$, where $\bar{H}=S O_{N_{0}}$. Take $u \in \mathscr{O}$, and put $\lambda=\left(2 a+\nu_{1}, \nu_{2}, \ldots, \nu_{N}\right)$. If we note that $n(\lambda)=n(\nu)$, by using Lemma 7.4 we have

$$
\operatorname{dim} Z_{H}(u)=\operatorname{dim} Z_{\bar{H}}(v)=n(\nu)=n(\lambda) .
$$

On the other hand, let $d_{\mathscr{O}}$ be as in (5.2.2). The previous computation shows that $d_{\mathscr{O}}=0$. Thus $R^{2 d_{\mathscr{O}}} f_{!} \overline{\mathscr{E}}_{1}=f_{!} \overline{\mathscr{E}}_{1}$. Since $\eta_{P}^{-1}\left(\mathscr{O}_{L}\right) \cap \mathscr{O}$ is open dense in $\eta^{-1}\left(\mathscr{O}_{L}\right)$, we see that $\left.f_{!} \overline{\mathscr{E}}_{1}\right|_{\mathscr{O}} \neq 0$. Hence by Theorem 5.2 (iii), the pair $(\mathscr{O}, \mathscr{E})$ belongs to $\left(L \subset P, \mathscr{O}_{L}, \mathscr{E}_{1}^{\dagger}\right)$ for some local system $\mathscr{E}$ on $\mathscr{O}$. Then by Lemma 8.19 , the Jordan type of $\mathscr{O}$ satisfies the condition (8.19.1). One can check that if $\lambda^{\prime} \neq \lambda$ satisfies the condition (8.19.1), then $n\left(\lambda^{\prime}\right)>n(\lambda)$. This implies, by (8.20.1), that the Jordan type of $\mathscr{O}$ is equal to $\lambda$. The proposition is proved.

We can now prove the following theorem, which is a counter-part of [L1, Theorem 9.2] in the symmetric space case.

Theorem 8.21. Let the notations be as in 8.18.

(i) The algebra $\mathscr{A}_{\mathscr{E}_{1}}$ is isomorphic to the group algebra $\overline{\mathbf{Q}}_{l}\left[S_{a}\right]$.

(ii) Let $\mathscr{E}_{0}=\mathscr{E}_{\tau}$ be the local system on $\mathscr{O}_{0}$ defined by $\tau=\left(\tau_{1}, \ldots, \tau_{N}\right)$ with $\tau_{i}=\sigma_{i}$ for $i=1, \ldots, N_{0}$, and $\tau_{i}=1$ for $i>N_{0}$. Then $\left(\mathscr{O}_{0}, \mathscr{E}_{0}\right)$ belongs to $\left(L \subset P, \mathscr{O}_{L}, \mathscr{E}_{1}^{\dagger}\right) . \mathscr{E}_{0}$ is the unique local system on $\mathscr{O}_{0}$ such that $\left(\mathscr{O}_{0}, \mathscr{E}_{0}\right)$ belongs to $\left(L \subset P, \mathscr{O}_{L}, \mathscr{E}_{1}^{\dagger}\right)$.

(iii) Under the isomorphism $\mathscr{A}_{\mathscr{E}_{1}} \simeq \overline{\mathrm{Q}}_{l}\left[S_{a}\right]$ in (i), $\left(\mathscr{O}_{0}, \mathscr{E}_{0}\right)$ corresponds to the unit representation of $S_{a}$. 
Proof. The statement of the theorem can be formulated by replacing $G$ by $L^{(i)}$. In the case where $i=0$ the claim of the theorem is trivial. By induction on $i$, we may assume that the claim holds for $i=a-1$, i.e., for $L_{H}^{(a-1)}=M_{H}$. Let $\left(\mathscr{O}_{0}^{\prime}, \mathscr{E}_{0}^{\prime}\right)$ be the pair in $\mathscr{N}_{M}$ defined similarly to $\left(\mathscr{O}_{0}, \mathscr{E}_{0}\right)$ for $H$. Let $\lambda^{\prime}$ be the Jordan type of $\mathscr{O}_{0}^{\prime}$. From the proof of Proposition 8.20, we know that there exists a pair $\left(\mathscr{O}_{0}, \mathscr{E}\right)$ which belongs to $\left(L \subset P, \mathscr{O}_{L}, \mathscr{E}_{1}^{\dagger}\right)$ for some local system $\mathscr{E}$ on $\mathscr{O}_{0}$. Let $\rho \in \mathscr{A}_{\mathscr{E}_{1}}^{\wedge}$ be the irreducible character corresponding to $\left(\mathscr{O}_{0}, \mathscr{E}\right)$. Also define $\rho^{\prime} \in\left(\mathscr{A}_{\mathscr{E}_{1}}^{\prime}\right)^{\wedge}$ as the character corresponding to $\left(\mathscr{O}_{0}^{\prime}, \mathscr{E}_{0}^{\prime}\right)$. Let $\rho^{\prime \prime} \in\left(\mathscr{A}_{\mathscr{E}_{1}}^{\prime}\right)^{\wedge}$ be a character appearing in the restriction of $\rho$ on $\mathscr{A}_{\mathscr{E} 1}^{\prime}$, and let $\left(\mathscr{O}^{\prime \prime}, \mathscr{E}^{\prime \prime}\right)$ be the corresponding pair in $\mathscr{N}_{M_{H}}$. By Corollary 6.11 and Proposition 8.7, the Jordan type $\lambda^{\prime \prime}$ of $\mathscr{O}^{\prime \prime}$ is obtained from $\lambda$ by the procedure $\left(\mathrm{A}_{i}^{\prime}\right)$ for some $i$. On the other hand, by Lemma 8.19, $\lambda^{\prime \prime}$ satisfies the condition (8.19.1). It follows that the multiplicity $\left\langle\rho, \rho^{\prime \prime}\right\rangle=0$ unless $\lambda^{\prime \prime}=\lambda^{\prime}$. Now assume that $\lambda^{\prime \prime}=\lambda^{\prime}$, i.e., $\mathscr{O}^{\prime \prime}=\mathscr{O}_{0}^{\prime}$. In this case, by our assumption, we have $\left(\mathscr{O}^{\prime \prime}, \mathscr{E}^{\prime \prime}\right)=\left(\mathscr{O}_{0}^{\prime}, \mathscr{E}_{0}^{\prime}\right)$, hence $\rho^{\prime \prime}=\rho^{\prime}$. We write $\mathscr{E}=\mathscr{E}_{\xi}$ for $\xi \in A_{H}(u)^{\wedge}$, and $\mathscr{E}_{0}^{\prime}=\mathscr{E}_{\tau^{\prime}}^{\prime}$ for $\tau^{\prime} \in A_{M_{H}}(v)$ with $v \in \mathscr{O}_{0}^{\prime}$. By Corollary 6.11, $\left\langle\rho, \rho^{\prime}\right\rangle$ coincides with the multiplicity of $\xi \otimes \tau^{\prime}$ in $\varepsilon_{u, v}$. Since $\left\langle\rho, \rho^{\prime}\right\rangle \neq 0$, by Proposition 8.16, we see that $\xi=\tau$ and that $\left\langle\rho, \rho^{\prime}\right\rangle=1$. This shows that $\mathscr{E}=\mathscr{E}_{0}$, and (ii) holds.

The above discussion shows that the restriction of $\rho$ on $\mathscr{A}_{\mathscr{E} 1}^{\prime}$ coincides with $\rho^{\prime}$. Since $\rho^{\prime}$ is a one-dimensional representation by our assumption, we see that $\rho$ is one-dimensional. Recall that $\mathscr{A}_{\mathscr{E}}$ is isomorphic to a twisted group algebra $\overline{\mathbf{Q}}_{l}\left[\mathscr{W}_{\mathscr{E}_{1}}\right]$ (see 3.6). By making use of the one-dimensional representation $\rho$, in a similar way as in the proof of Theorem 9.2 in [L1], we can construct an algebra isomorphism $\mathscr{A}_{\mathscr{E}_{1}} \stackrel{\sim}{\rightarrow} \overline{\mathbf{Q}}_{l}\left[\mathscr{W}_{\mathscr{E}_{1}}\right]$, where $\rho$ corresponds to the unit representation on $\mathscr{W}_{\mathscr{E}_{1}}$. But in the definition of $\mathscr{W}_{\mathscr{E}_{1}}$ in 3.6, $n \in N_{H}\left(L_{H}\right)$ induces a trivial automorphism $\operatorname{ad}(n)$ on $L_{H}$, hence acts trivially on $\mathscr{O}_{L}$ if the image of $n$ in $\mathscr{W}$ is contained in $\mathscr{W}_{1}$. It follows that $\mathscr{W}_{\mathscr{E}_{1}} \simeq S_{a}$. Thus (i) and (iii) holds. The theorem is proved.

Remark 8.22. In [L1, Proposition 9.5], the unipotent class corresponding to the sign representation of $N_{G}(L) / L$ was determined. In the symmetric space case, however, the behavior of the $H$-orbit corresponding to the sign representation of $S_{a}$ is more complicated. For the description of this $H$-orbit, one has to wait for the determination of the whole generalized Springer correspondence.

\section{Determination of the Generalized Springer Correspondence}

9.1. In order to obtain an exact parametrization of $H$-orbits in $G_{\text {uni }}^{\iota \theta}$ in the case where $N$ is even, we need some preliminaries. Assume that $N=2 n$. Let $P^{+}$be a $\theta$-stable parabolic subgroup of $G$ containing $B$ and $L^{+}$the $\theta$-stable Levi subgroup of $G$ containing $T$ such that $L_{H}^{+} \simeq G L_{n}$. Then we can write as

$$
L_{H}^{+}=\left\{\left(\begin{array}{cc}
a & 0 \\
0 & { }^{t} a^{-1}
\end{array}\right) \mid a \in G L_{n}\right\}, \quad L^{+\iota \theta}=\left\{\left(\begin{array}{cc}
a & 0 \\
0 & { }^{t} a
\end{array}\right) \mid a \in G L_{n}\right\} .
$$

Thus $L^{+\iota \theta}$ is in natural bijection with $G L_{n}$, and the conjugation action of $L_{H}^{+}$on $L^{+\iota \theta}$ coincides with the conjugation action of $G L_{n}$ on $G L_{n}$. Let $\mathscr{O}_{\lambda}^{\dagger}$ be the $L_{H}^{+}$-orbit in 
$L^{+\iota \theta}$ corresponding to the unipotent class of $G L_{n}$ corresponding to $\lambda \in \mathscr{P}_{n}$. Let $\mathscr{O}$ be the unique $H$-orbit in $G^{\iota \theta}$ such that $\eta_{P^{+}}^{-1}\left(\mathscr{O}_{\lambda}^{\dagger}\right) \cap \mathscr{O}$ is open dense in $\eta_{P^{+}}^{-1}\left(\mathscr{O}_{\lambda}^{\dagger}\right)$, where $\eta_{P^{+}}: P^{+\iota \theta} \rightarrow L^{+\iota \theta}$ is defined similarly as before. Similarly to the map $\pi: \widetilde{X} \rightarrow X$ in 3.4 , we define

$$
\begin{aligned}
& \tilde{X}_{\lambda}^{+}=\left\{\left(x, g P_{H}^{+}\right) \in G_{\mathrm{uni}}^{\iota \theta} \times H / P_{H}^{+}, \mid g^{-1} x g \in \eta_{P^{+}}^{-1}\left(\overline{\mathscr{O}}_{\lambda}^{\dagger}\right)\right\}, \\
& X_{\lambda}^{+}=\bigcup_{g \in H} g \eta_{P^{+}}^{-1}\left(\overline{\mathscr{O}}_{\lambda}^{\dagger}\right) g^{-1},
\end{aligned}
$$

and let $\pi_{\lambda}: \widetilde{X}_{\lambda}^{+} \rightarrow X_{\lambda}^{+}$be the first projection. Then $\pi_{\lambda}$ is proper surjective, and $X_{\lambda}^{+}$is an irreducible closed subset of $G^{\iota \theta}$. The following result is an analogue of Proposition 8.20.

Lemma 9.2. For $\lambda=\left(\lambda_{1}, \ldots, \lambda_{k}\right) \in \mathscr{P}_{n}$, put $2 \lambda=\left(2 \lambda_{1}, \ldots, 2 \lambda_{k}\right) \in \mathscr{P}_{N}$.

(i) $\mathscr{O}$ is the unique open dense orbit in $X_{\lambda}^{+}$.

(ii) The Jordan type of $\mathscr{O}$ is equal to $2 \lambda$.

(iii) $\operatorname{dim} \widetilde{X}_{\lambda}^{+}=\operatorname{dim} X_{\lambda}^{+}$.

Proof. The proof of (i) is similar to the proof of Proposition 8.20 (i). We show (ii) and (iii). First compute the dimension of $\widetilde{X}_{\lambda}^{+}$. Since $\widetilde{X}_{\lambda}^{+} \simeq H \times{ }^{P_{H}^{+}} \eta_{P^{+}}^{-1}\left(\overline{\mathscr{O}}_{\lambda}^{\dagger}\right)$,

$$
\begin{aligned}
\operatorname{dim} \widetilde{X}_{\lambda}^{+} & =\operatorname{dim} U_{P^{+}}^{\theta}+\operatorname{dim} \mathscr{O}_{\lambda}^{\dagger}+\operatorname{dim} U_{P^{+}}^{\iota \theta} \\
& =\operatorname{dim} U_{P^{+}}+\operatorname{dim} \mathscr{O}_{\lambda}^{\dagger} \\
& =2 n^{2}-n-2 n(\lambda)
\end{aligned}
$$

since $\operatorname{dim} U_{P^{+}}=n^{2}, \operatorname{dim} \mathscr{O}_{\lambda}^{\dagger}=n^{2}-n-2 n(\lambda)$ (known result for $G L_{n}$, see (7.3.1)). Since $\operatorname{dim} H=2 n^{2}-n$, and $\pi_{\lambda}$ is surjective, we have

$$
\operatorname{dim} X_{\lambda}^{+} \leq \operatorname{dim} H-2 n(\lambda)
$$

If $\mathscr{O}^{\prime}$ is an $H$-orbit of type $2 \lambda$, then $\operatorname{dim} \mathscr{O}^{\prime}=\operatorname{dim} H-2 n(\lambda)$ by Lemma 7.4. Hence in order to prove (ii) and (iii), it is enough to show that $X_{\lambda}^{+}$contains an element of Jordan type $2 \lambda$. Choose a basis $e_{1}, \ldots, e_{n}, f_{1}, \ldots, f_{n}$ of $V$ as in 1.7. Consider the following map $x: V \rightarrow V$;

$$
\begin{aligned}
& f_{1} \mapsto f_{2} \mapsto \cdots \mapsto f_{\lambda_{1}} \mapsto e_{\lambda_{1}} \mapsto e_{\lambda_{1}-1} \mapsto \cdots \mapsto e_{1} \mapsto 0, \\
& f_{\lambda_{1}+1} \mapsto f_{\lambda_{1}+2} \mapsto \cdots \mapsto f_{\lambda_{1}+\lambda_{2}} \mapsto e_{\lambda_{1}+\lambda_{2}} \mapsto e_{\lambda_{1}+\lambda_{2}-1} \mapsto \cdots \mapsto e_{\lambda_{1}+1} \mapsto 0, \\
& \cdots \cdots \cdots \\
& f_{\lambda_{1}+\cdots+\lambda_{k-1}+1} \mapsto \cdots \mapsto f_{n} \mapsto e_{n} \mapsto \cdots \mapsto e_{\lambda_{1}+\cdots+\lambda_{k-1}+1} \mapsto 0 .
\end{aligned}
$$

Then $u=x+1 \in \eta_{P^{+}}^{-1}\left(\mathscr{O}_{\lambda}^{\dagger}\right)$ and the Jordan type of $u$ is $2 \lambda$. Thus (ii) and (iii) hold. The lemma is proved. 
9.3. For an even partition $2 \lambda$ of $N$, we denote by $\mathscr{O}_{2 \lambda}^{+}$the unique open dense orbit in $X_{\lambda}^{+}$given in Lemma 9.2. We denote by $\mathscr{O}_{2 \lambda}^{-}$another $H$-orbit contained in $\mathscr{O}_{2 \lambda}$. Note that (9.2.1) gives an explicit representative of the $H$-orbit $\mathscr{O}_{2 \lambda}^{+}$. In particular, all the $\mathscr{O}_{2 \lambda}^{+}$are contained in $X_{\text {uni }}=\bigcup_{g \in H} g U^{\iota \theta} g^{-1}$. Hence $\mathscr{O}_{(2 n)}^{+}$coincides with the unique open dense orbit $\mathscr{O}_{0}$ in $X_{\text {uni }}$ given in Proposition 8.20.

Let $t_{n} \in N_{G^{\theta}}(T)-N_{H}(T)$ be as in 1.9. Then $t_{n} \mathscr{O}_{2 \lambda}^{+} t_{n}^{-1}=\mathscr{O}_{2 \lambda}^{-}$. Put $P^{-}=t_{n} P^{+} t_{n}^{-1}$ and $L^{-}=t_{n} L^{+} t_{n}^{-1}$. Replacing $P^{+}, L^{+}$by $P^{-}, L^{-}$, we can define a variety $X_{\lambda}^{-}$ similarly to $X_{\lambda}^{+}$. Then $\mathscr{O}_{2 \lambda}^{-}$is characterized as the unique open dense orbit contained in $X_{\lambda}^{-}$. The representatives of $\mathscr{O}_{2 \lambda}^{-}$are obtained by applying $\operatorname{ad}\left(t_{n}\right)$ on $x$ in (9.2.1).

We have a refinement of Proposition 8.7.

Lemma 9.4. Assume that $\lambda, \lambda^{\prime}$ are even partitions. Let $Y_{u, v}$ be as in Proposition 8.7, where $u \in \mathscr{O}_{\lambda}^{\varepsilon}, v \in \mathscr{O}_{\lambda^{\prime}}^{\varepsilon^{\prime}}$ with $\varepsilon, \varepsilon^{\prime} \in\{1,-1\}$. If $I\left(Y_{u, v}\right) \neq \emptyset$, then we have $\varepsilon=\varepsilon^{\prime}$.

Proof. Assume that $u \in \mathscr{O}_{\lambda}^{+}$. We choose a representative $u$ such that $x=u-1$ is given as in (9.2.1). Then $\bar{x} \in \mathscr{O}_{\lambda^{\prime}}^{ \pm}$is obtained from $x$, for example, by replacing

$$
f_{1} \mapsto f_{2} \mapsto \cdots f_{\lambda_{1}} \mapsto e_{\lambda_{1}} \mapsto \cdots \mapsto e_{2} \mapsto e_{1} \mapsto 0
$$

by

$$
f_{2} \mapsto \cdots \mapsto f_{\lambda_{1}} \mapsto e_{\lambda_{1}} \mapsto \cdots \mapsto e_{2} \mapsto 0
$$

Thus $v=\bar{x}+1$ is contained in a similar variety $X_{\lambda^{\prime}}^{+}$as $X_{\lambda}^{+}$defined for $\lambda^{\prime}$. Hence $v \in \mathscr{O}_{\lambda^{\prime}}^{+}$. If $u \in \mathscr{O}_{\lambda}^{-}$, we can apply the same argument by replacing $X_{\lambda}^{+}$by $X_{\lambda}^{-}$, and obtain that $v \in \mathscr{O}_{\lambda^{\prime}}^{-}$. The lemma is proved.

9.5. Assume that $N \geq 1$ is an odd integer. A pair $(\lambda, \tau)$ is called a signed partition if $\lambda=\left(\lambda_{1}, \ldots, \lambda_{N}\right)$ is a partition of $N$, and if $\tau=\left(\tau_{1}, \ldots, \tau_{N}\right)$ satisfies the condition (cf. 8.17) that

(i) $\tau_{i}= \pm 1$, and $\tau_{i}=1$ if $\lambda_{i}=0$,

(ii) $\tau_{i}=\tau_{j}$ if $\lambda_{i}=\lambda_{j}$,

(iii) $\tau_{i_{0}}=1$ where $i_{0}$ is the index such that $\lambda_{i_{0}}$ is the largest odd number among $\lambda_{1}, \ldots, \lambda_{N}$.

We denote by $\Psi_{N}$ the set of signed partitions of $N$. Note that, by Proposition 7.8 (i), the set of $H$-orbits in $G_{\text {uni }}^{\iota \theta}$ is parametrized by $\mathscr{P}_{N}$. We denote by $\mathscr{O}_{\lambda}$ the $H$-orbit corresponding to $\lambda \in \mathscr{P}_{N}$. Then as in 8.17, $H$-equivariant simple local system on $\mathscr{O}_{\lambda}$ can be expressed as $\mathscr{E}_{\tau}$ for $(\lambda, \tau) \in \Psi_{N}$, and the map $(\lambda, \tau) \mapsto\left(\mathscr{O}_{\lambda}, \mathscr{E}_{\tau}\right)$ gives a bijection $\Psi_{N} \underset{\sim}{\rightarrow} \mathscr{N}_{G}$ if $N \geq 3$.

Next assume that $N \geq 0$ is an even integer. In this case, for each partition $\lambda \in \mathscr{P}_{N}$, we prepare two copies $\lambda^{ \pm}$, and assume that $\lambda^{+}=\lambda^{-}$if $\lambda$ is not an even partition. A signed partition $\left(\lambda^{ \pm}, \tau\right)$ is defined similarly as above, for each $\lambda^{+}$and $\lambda^{-}$. We denote by $\Psi_{N}$ the set of signed partitions of $N$. Note that if $N=0$, we regard the empty partition $\emptyset$ as an even partition, so we consider $\left(\emptyset^{+}, 1\right)$ and $\left(\emptyset^{-}, 1\right)$. By Proposition 7.8 (ii), the set of $G^{\theta}$-orbits in $G_{\text {uni }}^{\iota \theta}$ is parametrized by $\mathscr{P}_{N}$. $G^{\theta}$-orbit $\mathscr{O}_{\lambda}$ is a single $H$-orbit unless $\lambda$ is an even partition, in which case, $\mathscr{O}_{\lambda}$ splits into two $H$-orbits. By 9.3 , we denote those two $H$-orbits by $\mathscr{O}_{\lambda}^{+}$and $\mathscr{O}_{\lambda}^{-}$. By abuse of the notation, we denote $\mathscr{O}_{\lambda}$ by $\mathscr{O}_{\lambda}^{+}=\mathscr{O}_{\lambda}^{-}$if $\lambda$ is not an even partition. Thus we have a bijective map $\Psi_{N} \stackrel{\sim}{\rightarrow} \mathscr{N}_{G}$ by $\left(\lambda^{ \pm}, \tau\right) \mapsto\left(\mathscr{O}_{\lambda}^{ \pm}, \mathscr{E}_{\tau}\right)$, if $N \geq 2$. 
9.6. Assume that $N$ is odd. Take integers $N_{0} \geq 1, a \geq 0$ such that $N=2 a+N_{0}$. For a fixed $\xi=(\nu, \sigma) \in \Psi_{N_{0}}$, we define a map $\Gamma_{\xi}: \mathscr{P}_{a} \rightarrow \Psi_{N}$ as follows; write $\nu=\left(\nu_{1}, \ldots, \nu_{N}\right), \sigma=\left(\sigma_{1}, \ldots, \sigma_{N}\right)$ by putting $\nu_{i}=0, \sigma_{i}=1$ for $i>N_{0}$. For each $\mu=\left(\mu_{1}, \ldots, \mu_{N}\right) \in \mathscr{P}_{a}$, define integers $\lambda_{1}, \ldots, \lambda_{N}$ by

$$
\lambda_{i}=\nu_{i}+2 \mu_{i} \quad \text { for } i=1, \ldots, N \text {. }
$$

Then $\lambda=\left(\lambda_{1}, \ldots, \lambda_{N}\right) \in \mathscr{P}_{N}$, and the pair $(\lambda, \sigma)$ gives a well-defined element in $\Psi_{N}$. By definition, $\Gamma_{\xi}(\mu)=(\lambda, \sigma)$. In the case where $N$ is even, the map $\Gamma_{\xi}: \mathscr{P}_{a} \rightarrow \Psi_{N}$ is defined similarly, but we consider $\xi^{ \pm}=\left(\nu^{ \pm}, \sigma\right)$, and put $\Gamma_{\xi^{ \pm}}(\mu)=\left(\lambda^{ \pm}, \sigma\right)$.

Let $N \geq 0$ be an integer. An element $(\lambda, \tau) \in \Psi_{N}\left(\right.$ or $\left.\left(\lambda^{ \pm}, \tau\right) \in \Psi_{N}\right)$ is called cuspidal if

(i) $\lambda_{i}-\lambda_{i+1} \leq 2$ for $i=1, \ldots, N$ (here we put $\lambda_{N+1}=0$ ),

(ii) If $\lambda_{i}-\lambda_{i+1}=2$, then $\tau_{i} \neq \tau_{i+1}$.

We denote by $\mathscr{C}_{N}$ the set of triples $\left(N_{0}, \nu, \sigma\right)$ such that $N-N_{0} \in 2 \mathbf{Z}_{\geq 0}$ and $\xi=(\nu, \sigma) \in \Psi_{N_{0}}$ is a cuspidal element. For $c \in \mathscr{C}_{N}$, we denote by $\Psi_{N}^{(c)}$ the image of $\Gamma_{\xi}: \mathscr{P}_{a} \rightarrow \Psi_{N}$, where $a=\left(N-N_{0}\right) / 2$. Clearly $\Gamma_{\xi}$ gives a bijection $\mathscr{P}_{a} \underset{\rightarrow}{\rightarrow} \Psi_{N}^{(c)}$. We also denote by $\Psi_{N}^{(0)}$ the set of cuspidal elements in $\Psi_{N}$.

\section{Proposition 9.7. There exists a partition}

$$
\Psi_{N}=\coprod_{c \in \mathscr{C}_{N}} \Psi_{N}^{(c)}
$$

Proof. For simplicity we assume that $N$ is odd. The case where $N$ is even is dealt similarly. Assume that $(\lambda, \tau) \in \Psi_{N}$ is not cuspidal. Then there exists some $i$ such that $\lambda_{i}>\lambda_{i+1}+2$ or that $\lambda_{i}=\lambda_{i+1}+2$ with $\tau_{i}=\tau_{i+1}$. Put $\lambda^{\prime}=\left(\lambda_{1}^{\prime}, \ldots, \lambda_{N}^{\prime}\right) \in \mathscr{P}_{N-2}$, where $\lambda_{i}^{\prime}=\lambda_{i}-2, \lambda_{j}^{\prime}=\lambda_{j}$ for $j \neq i$. If we put $\tau^{\prime}=\tau$, we have $\left(\lambda^{\prime}, \tau^{\prime}\right) \in \Psi_{N-2}$ by our assumption. By induction on $N$, we may assume (9.7.1) holds for $\Psi_{N-2}$. Hence there exists $c=\left(N_{0}, \nu, \sigma\right) \in \mathscr{C}_{N-2}$ such that $\left(\lambda^{\prime}, \tau^{\prime}\right) \in \Psi_{N-2}^{(c)}$. In particular, $\left(\lambda^{\prime}, \tau^{\prime}\right)=\Gamma_{\xi}\left(\mu^{\prime}\right)$ for some $\mu^{\prime} \in \mathscr{P}_{a}$, where $\xi=(\nu, \sigma)$ and $a=\left(N-2-N_{0}\right) / 2$. Here $\mu^{\prime}=\left(\mu_{1}^{\prime}, \ldots, \mu_{N}^{\prime}\right)$ satisfies the condition that $\mu_{i-1}^{\prime} \geq \mu_{i}^{\prime}+1$, and if we define $\mu=\left(\mu_{1}, \ldots, \mu_{N}\right) \in \mathscr{P}_{a+1}$ by $\mu_{i}=\mu_{i}^{\prime}+1, \mu_{j}=\mu_{j}^{\prime}$ for $j \neq i,(\lambda, \tau)=\Gamma_{\xi}(\mu)$. Hence $(\lambda, \tau) \in \Psi_{N}^{(c)}$. $c$ is determined by $(\lambda, \tau)$ uniquely. In fact, suppose that $(\lambda, \tau) \in \Psi_{N}^{\left(c^{\prime}\right)}$ for another $c^{\prime}$. The above argument shows, since $(\nu, \sigma)$ is cuspidal, that $\left(\lambda^{\prime}, \tau^{\prime}\right) \in \Psi_{N-2}^{\left(c^{\prime}\right)}$. Hence by induction on $N$, we have $c=c^{\prime}$.

9.8. We have a natural parametrization of $S_{a}^{\wedge}$ by $\mathscr{P}_{a}$. We denote by $\rho_{\mu}$ the irreducible representation of $S_{a}$ corresponding to $\mu \in \mathscr{P}_{a}$. (Here the partition $(a)$ corresponds to the unit representation, $\left(1^{a}\right)$ corresponds to the sign representation.)

Under the identification $\Psi_{N} \underset{\sim}{\sim} \mathscr{N}_{G}$ in 9,5 , we denote by $\mathscr{N}_{G}^{(0)}$ the image of $\Psi_{N}^{(0)}$. We also denote by $\mathscr{N}_{G}^{(c)}$ the subset of $\mathscr{N}_{G}$ which is the image of $\Psi_{N}^{(c)}$. For each 
$c=\left(N_{0}, \nu, \sigma\right) \in \mathscr{C}_{N}$ with $\xi=(\nu, \sigma)$, the map $\Gamma_{\xi}$ induces a bijection $\widetilde{\Gamma}_{c}: S_{a}^{\wedge} \underset{\rightarrow}{N_{G}^{(c)}}$, combined with $\mathscr{P}_{a} \simeq S_{a}^{\wedge}$. Thus, by Proposition 9.7 , we have a bijective map

$$
\widetilde{\Gamma}: \coprod_{c \in \mathscr{C}_{N}} S_{a}^{\wedge} \leadsto \coprod_{c \in \mathscr{C}_{N}} \mathscr{N}_{G}^{(c)}=\mathscr{N}_{G}
$$

where $a=\left(N-N_{0}\right) / 2$ for $c=\left(N_{0}, \nu, \sigma\right) \in \mathscr{C}_{N}$.

Recall the definition of $\mathscr{N}_{G}^{(\xi)}$ in 5.7 for $\xi=\left(L \subset P, \mathscr{O}_{L}, \mathscr{E}_{1}^{\dagger}\right) \in \mathscr{S}_{G}$. The following result gives a combinatorial description of the generalized Springer correspondence for the symmetric space associated to orthogonal groups.

Theorem 9.9. Let the notations be as above.

(i) $\mathscr{N}_{G}^{(0)}$ coincides with the set of cuspidal pairs in $\mathscr{N}_{G}$.

(ii) $c=\left(N_{0}, \nu, \sigma\right) \mapsto \xi=\left(L \subset P, \mathscr{O}_{L}, \mathscr{E}_{1}^{\dagger}\right)$ gives a bijection $\mathscr{C}_{N} \underset{\rightarrow}{\rightarrow} \mathscr{S}_{G}$ such that $\mathscr{N}_{G}^{(c)}=\mathscr{N}_{G}^{(\xi)}$, where $L$ is such that $L_{H} \simeq\left(G L_{1}\right)^{a} \times S O_{N_{0}}$, and $\left(\mathscr{O}_{L}, \mathscr{E}_{1}^{\dagger}\right)=$ $\left(\mathscr{O}_{\nu}, \mathscr{E}_{\sigma}^{\dagger}\right)$.

(iii) The map $\widetilde{\Gamma}$ in (9.8.1) gives a bijection in Theorem 5.2 (ii).

Proof. We prove the theorem by induction on $N$. In the case where $N=2,3$, the claim (iii) is verified directly, see Appendix. First consider the case where $N$ is odd. Assume that the theorem holds for $L \neq G$. We show (iii). Choose $\left(L \subset P, \mathscr{O}_{L}, \mathscr{E}_{1}^{\dagger}\right) \in \mathscr{S}_{G}$ with $L \neq G$. By (i) for $L$, we can attach $\left(N_{0}, \nu, \sigma\right) \in \mathscr{C}_{N}$ to $\left(L \subset P, \mathscr{O}_{L}, \mathscr{E}_{1}^{\dagger}\right)$. Take $\rho=\rho_{\mu} \in S_{a}^{\wedge}$, and let $(\mathscr{O}, \mathscr{E}) \in \mathscr{N}_{G}$ be the pair which belongs to $\left(L \subset P, \mathscr{O}_{L}, \mathscr{E}_{1}^{\dagger}\right)$ corresponding to $\rho$. Put $(\lambda, \tau)=\Gamma_{\xi}(\mu)$ for $\xi=(\nu, \sigma)$. In order to prove (iii), it is enough to see that $\mathscr{O}=\mathscr{O}_{\lambda}, \mathscr{E}=\mathscr{E}_{\tau}$. Let $Q_{H}$ be the maximal parabolic subgroup of $H$ containing $P$ and $M_{H}$ its Levi subgroup containing $L$ as in Section 8. Let $\rho^{\prime}=\rho_{\mu^{\prime}}$ be an irreducible character of $S_{a-1}$, and $\left(\mathscr{O}^{\prime}, \mathscr{E}^{\prime}\right) \in \mathscr{N}_{M}$ the pair which belongs to $\left(L \subset M \cap P, \mathscr{O}_{L}, \mathscr{E}_{1}^{\dagger}\right)$ corresponding to $\rho^{\prime}$. By induction hypothesis, one can write as $\left(\mathscr{O}^{\prime}, \mathscr{E}^{\prime}\right)=\left(\mathscr{O}_{\lambda^{\prime}}^{\prime}, \mathscr{E}_{\tau^{\prime}}^{\prime}\right)$ with $\left(\lambda^{\prime}, \tau^{\prime}\right)=\Gamma_{\xi}\left(\mu^{\prime}\right)$. By Corollary 6.11 and Proposition 8.7, if $\left\langle\rho, \rho^{\prime}\right\rangle \neq 0$, then the Jordan type of $\mathscr{O}$ is obtained from $\lambda^{\prime}$ by the inverse operation of $\left(\mathrm{A}_{i}^{\prime}\right)$ for some $i$. This is true for any $\rho^{\prime}$ such that $\left\langle\rho, \rho^{\prime}\right\rangle \neq 0$. In the case where $a \geq 3$, this condition determines the Jordan type of $\mathscr{O}$ uniquely, and we have $\mathscr{O}=\mathscr{O}_{\lambda}$. Moreover, in this case, by Proposition 8.16, $\mathscr{E}$ is determined uniquely from $\mathscr{E}_{\tau^{\prime}}^{\prime}$, namely we have $\mathscr{E}=\mathscr{E}_{\tau}$. Thus we have proved (iii) for $a \geq 3$.

Now assume that $a=1$. In this case, $S_{1}=\{1\}$, and $\left.\pi_{*} K_{\overline{\mathscr{E}}_{1}}\right|_{X_{\text {uni }}}=\operatorname{IC}\left(\overline{\mathscr{O}}_{\lambda^{\prime}}, \mathscr{E}_{\tau^{\prime}}\right)$ up to shift, where $\lambda^{\prime}=\left(\nu_{1}+2, \nu_{2}, \ldots, \nu_{N}\right), \tau^{\prime}=\left(\sigma_{1}, \ldots, \sigma_{N}\right)$ by Theorem 8.21. Hence the claim holds. Next assume that $a=2$. In this case, $S_{2}^{\wedge}$ has two representations, the unit representation $\rho_{(2)}$ and the sign representation $\rho_{\left(1^{2}\right)}$. By Lemma 8.19, the possibility for $(\mathscr{O}, \mathscr{E})$ belonging to $\left(L \subset P, \mathscr{O}_{L}, \mathscr{E}_{1}^{\dagger}\right)$ is only $\mathscr{O}=\mathscr{O}_{\lambda}$ with $\lambda=\left(\nu_{1}+\right.$ $\left.4, \nu_{2}, \ldots, \nu_{N}\right)$ or $\lambda=\left(\nu_{1}+2, \nu_{1}+2, \nu_{3}, \ldots, \nu_{N}\right)$. By Theorem 8.21, $\rho_{(2)}$ corresponds to $\left(\mathscr{O}_{0}, \mathscr{E}_{0}\right)=\left(\mathscr{O}_{\lambda}, \mathscr{E}_{\tau}\right)$, where $\lambda=\left(\nu_{1}+4, \nu_{2}, \ldots, \nu_{N}\right)$ and $\tau=\left(\sigma_{1}, \ldots, \sigma_{N}\right)$. Hence $\rho_{\left(1^{2}\right)}$ corresponds to $\left(\mathscr{O}_{\lambda}, \mathscr{E}_{\tau}\right)$ with $\lambda=\left(\nu_{1}+2, \nu_{2}+2, \nu_{3}, \ldots, \nu_{N}\right)$. The restriction of $\rho_{\left(1^{2}\right)}$ on $S_{1}$ is $\rho_{(1)}$ which corresponds to $\left(\mathscr{O}_{\lambda^{\prime}}, \mathscr{E}_{\tau^{\prime}}\right)$. By a similar argument as above, 
$\tau$ is determined from $\tau^{\prime}$, namely we see that $(\lambda, \tau)=\Gamma_{\xi}\left(1^{(2)}\right)$. Thus the claim holds for $a=2$. Hence (iii) is proved for odd $N$.

Next consider the case where $N$ is even. We choose $\left(L \subset P, \mathscr{O}_{L}, \mathscr{E}_{1}^{\dagger}\right) \in \mathscr{S}_{G}$. Assume that the Jordan type of $\mathscr{O}_{L}$ is not an even partition. Then a similar argument as before works. (Note that in that case the Jordan type of $\mathscr{O}$ is not even for any $(\mathscr{O}, \mathscr{E})$ belonging to $\left(L \subset P, \mathscr{O}_{L}, \mathscr{E}_{1}^{\dagger}\right)$ by Lemma 8.19.) Thus we assume that the Jordan type of $\mathscr{O}_{L}$ is even. Then by the same reason, the Jordan type of $\mathscr{O}$ is even for such $(\mathscr{O}, \mathscr{E})$. The previous argument shows that if $(\mathscr{O}, \mathscr{E})$ corresponds to $\rho=\rho_{\mu} \in S_{a}^{\wedge}$, then the Jordan type of $\mathscr{O}$ is $\lambda$, and $\mathscr{E}=\mathscr{E}_{\tau}$ for $\Gamma_{\xi}(\mu)=(\lambda, \tau)$. But we have to show that $\mathscr{O}=\mathscr{O}_{\lambda}^{ \pm}$if $\Gamma_{\xi^{ \pm}}(\mu)=\left(\lambda^{ \pm}, \tau\right)$. This is done by using Lemma 9.4. Thus (iii) is proved for even $N$ similarly as above.

We show (i) and (ii). Assume that $N$ is even. If $N_{0}=0$, then $a=n$, and there exists two triples $c^{+}, c^{-} \in \mathscr{C}_{N}$, where $c^{ \pm}=\left(0, \emptyset^{ \pm}, 1\right)$. We have $\Psi^{\left(c^{ \pm}\right)} \simeq \mathscr{P}_{n}$. On the other hand, $N_{0}=0$ corresponds to the case where $L=T, \mathscr{O}_{L}=\{1\}$ and $\mathscr{E}_{1}^{\dagger}=\overline{\mathbf{Q}}_{l}$ : the constant sheaf. Hence there exist two triples $\left(T \subset B,\{1\}, \overline{\mathbf{Q}}_{l}\right),(T \subset$ $\left.B_{1},\{1\}, \overline{\mathbf{Q}}_{l}\right) \in \mathscr{S}_{G}$, where $B_{1}=t_{n} B t_{n}^{-1}$. By (iii), the Springer correspondence gives a bijection $\Psi^{\left(c^{ \pm}\right)} \simeq \mathscr{N}_{G}^{\left(c^{ \pm}\right)}$. For other cases (for any $\left.N\right), c=\left(N_{0}, \nu, \sigma\right) \in \mathscr{C}_{N}$ determines a unique triple $\left(L \subset P, \mathscr{O}_{L}, \mathscr{E}_{1}^{\dagger}\right) \in \mathscr{S}_{G}$. By (iii), and by Proposition 9.7, this proves (i). (ii) follows from (i). The theorem is proved.

9.10. Following the discussion in $[\mathrm{L} 1,10.6]$, we shall give a formula which describes the cardinality of $\Psi_{N}^{(0)}$, namely, the number of the cuspidal pairs $(\mathscr{O}, \mathscr{E})$ in $\mathscr{N}_{G}$. Note that the computation below is much simpler compared to the case of generalized Springer correspondence for orthogonal groups.

Let $t$ be an indeterminate. Recall that the partition function $p(n)=\left|\mathscr{P}_{n}\right|$ is defined by the formula $\prod_{i=1}^{\infty}\left(1-t^{i}\right)^{-1}=\sum_{n \geq 0} p(n) t^{n}$. We define functions $q_{1}(n), q_{2}(n)$ for $n \in \mathbf{Z}_{\geq 0}$ as follows;

$$
\begin{aligned}
& \prod_{i=1}^{\infty}\left(1+t^{i}\right)^{2}=\sum_{n \geq 0} q_{1}(n) t^{n} \\
& \prod_{i=1}^{\infty}\left(1+t^{2 i}\right)=\sum_{n \geq 0} q_{2}(n) t^{n} .
\end{aligned}
$$

Proposition 9.11. The cardinality $\left|\Psi_{N}^{(0)}\right|=\left|\mathscr{N}_{G}^{(0)}\right|$ of cuspidal pairs is given by the formula,

$$
\left|\Psi_{N}^{(0)}\right|= \begin{cases}\frac{1}{2} q_{1}(N) & \text { if } N \geq 3: \text { odd }, \\ \frac{1}{2} q_{1}(N)+\frac{3}{2} q_{2}(N) & \text { if } N \geq 2: \text { even. }\end{cases}
$$


Proof. Let $x_{N}$ be the number of elements in $\Psi_{N}$, and $x_{N}^{\prime}$ (resp. $x_{N}^{\prime \prime}$ ) the number of elements $(\lambda, \tau) \in \Psi_{N}$ such that $\lambda$ is not an even partition (resp. even partition). Hence $x_{N}=x_{N}^{\prime}+x_{N}^{\prime \prime}$. Then as in $[\mathrm{L} 1,10.6]$,

$$
\begin{aligned}
1+\sum_{N \geq 1}\left(2 x_{N}^{\prime}+x_{N}^{\prime \prime} / 2\right) t^{N} & =\sum_{i_{1}, i_{2}, \cdots \geq 0} 2^{\sharp\left\{a: i_{a}>0\right\}} t^{1 i_{1}+2 i_{2}+3 i_{3}+\cdots} \\
& =\left(1+\sum_{i_{1} \geq 1} 2 t^{i_{1}}\right)\left(1+\sum_{i_{2} \geq 1} 2 t^{i_{2}}\right) \cdots \\
& =\frac{1+t}{1-t} \frac{1+t^{2}}{1-t^{2}} \cdots \\
& =\prod_{i=1}^{\infty}\left(1-t^{2 i}\right)^{-1} \prod_{i=1}^{\infty}\left(1+t^{i}\right)^{2} .
\end{aligned}
$$

It follows that

$$
2 x_{N}^{\prime}+x_{N}^{\prime \prime} / 2=\sum_{0 \leq a \leq N / 2} p(a) q_{1}(N-2 a)
$$

By a similar computation shows that

$$
\begin{aligned}
1+\sum_{N \geq 2 ; \text { even }}\left(x_{N}^{\prime \prime} / 2\right) t^{N} & =\sum_{i_{1}, i_{2}, \cdots \geq 0} 2^{\sharp\left\{a: i_{a}>0\right\}} t^{2\left(1 i_{1}+2 i_{2}+\cdots\right)} \\
& =\left(1+\sum_{i_{1} \geq 1} 2 t^{2 i_{1}}\right)\left(1+\sum_{i_{2} \geq 1} 2 t^{4 i_{2}}\right) \cdots \\
& =\frac{1+t^{2}}{1-t^{2}} \frac{1+t^{4}}{1-t^{4}} \cdots \\
& =\prod_{i=1}^{\infty}\left(1-t^{2 i}\right)^{-1} \prod_{i=1}^{\infty}\left(1+t^{2 i}\right) .
\end{aligned}
$$

Hence we have

$$
x_{N}^{\prime \prime} / 2=\sum_{0 \leq a \leq N / 2} p(a) q_{2}(N-2 a) .
$$

Now assume that $N$ is even. Then by (9.11.1) and (9.11.2), we have

$$
x_{N}=x_{N}^{\prime}+x_{N}^{\prime \prime}=\sum_{0 \leq a \leq N / 2} p(a)\left\{\frac{1}{2} q_{1}(N-2 a)+\frac{3}{2} q_{2}(N-2 a)\right\} .
$$

On the other hand, by the generalized Springer correspondence (Theorem 9.9), we have 


$$
\left|\Psi_{N}\right|=\sum_{0 \leq a \leq N / 2}\left|S_{a}^{\wedge}\right|\left|\Psi_{N-2 a}^{(0)}\right| .
$$

Then by induction on $N$, we obtain the formula for $\left|\Psi_{N}^{(0)}\right|$. The case where $N$ is odd si similar (in this case, $x_{N}^{\prime \prime}=0$ ).

\section{INDUCTION}

10.1. We consider the group $G$ and $\theta: G \rightarrow G$ as in (2.1.4). We fix a $\theta$ stable pair $(B, T)$ of a Borel subgroup $B$ and a maximal torus of $G$. Let $P$ be a $\theta$-stable parabolic subgroup of $G$ containing $B$ and $L$ the $\theta$-stable Levi subgroup of $P$ containing $T$. As before, let $\eta_{P}: P^{\iota \theta} \rightarrow L^{\iota \theta}$ be the natural projection. Consider the following diagram

$$
L^{\iota \theta} \stackrel{\psi}{\longleftarrow} \widehat{X}^{P} \stackrel{\varphi^{\prime}}{\longrightarrow} \widetilde{X}^{P} \stackrel{\varphi^{\prime \prime}}{\longrightarrow} G^{\iota \theta}
$$

where

$$
\begin{aligned}
& \widehat{X}^{P}=\left\{(x, g) \in G^{\iota \theta} \times H \mid g^{-1} x g \in P^{\iota \theta}\right\}, \\
& \widetilde{X}^{P}=\left\{\left(x, g P_{H}\right) \in G^{\iota \theta} \times H / P_{H} \mid g^{-1} x g \in P^{\iota \theta}\right\},
\end{aligned}
$$

and $\varphi^{\prime}:(x, g) \mapsto\left(x, g P_{H}\right), \varphi^{\prime \prime}:\left(x, g P_{H}\right) \mapsto x$, and $\psi:(x, g) \mapsto \eta_{P}\left(g^{-1} x g\right)$. Then $\varphi^{\prime}, \psi$ are smooth with connected fibres. Moreover, $H \times P_{H}$ acts on $\widehat{X}^{P}$ by $(h, p)$ : $(x, g) \mapsto\left(h x h^{-1}, h g p^{-1}\right)$, and $H$ acts on $\widetilde{X}^{P}$ by $h:\left(x, g P_{H}\right) \mapsto\left(h x h^{-1}, h g P_{H}\right)$. $\varphi^{\prime}, \varphi^{\prime \prime}$ are $H$-equivariant, and $\psi$ is $H \times P_{H^{-}}$equivariant with respect to the trivial action of $H$ and the action of $P_{H}$ on $L^{\iota \theta}$ induced from the map $P_{H} \rightarrow L_{H}$.

Let $K$ be an $L_{H}$-equivariant perverse sheaf on $L^{\iota \theta}$, which is regarded as an $H \times P_{H}$-equivariant perverse sheaf. Since $\psi$ is smooth with connected fibre, there exists a perverse sheaf $\psi^{*} K[\alpha]$ on $\widehat{X}^{P}$, where $\alpha$ is the dimension of the fibre. $\psi^{*} K[\alpha]$ is $H \times P_{H}$-equivariant, and since $\varphi^{\prime}$ is a locally trivial principal $P_{H}$-bundle, there exits a perverse sheaf $K_{1}$ on $\widetilde{X}^{P}$ such that $\psi^{*} K[\alpha] \simeq \varphi^{\prime *} K_{1}[\beta]$, where $\beta=\operatorname{dim} P_{H}$. We define ind $K=\varphi_{!}^{\prime \prime} K_{1}$. Since $\varphi^{\prime \prime}$ is proper, ind $K$ is a semisimple complex on $G^{\iota \theta}$. Since $K_{1}$ is $H$-equivariant, ind $K$ is $H$-equivariant. We also write ind $K=\operatorname{ind}_{P}^{G} K$. ind $K$ is an analogue of the induction functor of the character sheaves ([L2]).

Let $Q$ be a $\theta$-stable parabolic subgroup of $G$ containing $P$, and $M$ the $\theta$-stable Levi subgroup of $Q$ containing $L$. Then $M \cap P$ is a $\theta$-stable parabolic subgroup of $M$ with Levi subgroup $L$. Thus one can define functors $\operatorname{ind}_{M \cap P}^{M}$ and $\operatorname{ind}_{Q}^{G}$. The following transitivity property can be proved in a similar way as in [L2, Proposition $4.2]$.

Proposition 10.2. Let $K$ be an $L_{H}$-equivariant perverse sheaf on $L^{\iota \theta}$. Assume that $\operatorname{ind}_{M \cap P}^{M} K$ is an $M$-equivariant perverse sheaf on $M^{\iota \theta}$. Then we have 


$$
\operatorname{ind}_{Q}^{G}\left(\operatorname{ind}_{M \cap P}^{M} K\right) \simeq \operatorname{ind}_{P}^{G} K
$$

10.3. Returning to the original setting, we consider $G=G L_{N}$, and let $P$ be the $\theta$-stable parabolic subgroup such that $L_{H} \simeq\left(G L_{1}\right)^{a} \times S O_{N_{0}}$, where $N=$ $N_{0}+2 a$. Let $Q$ be the $\theta$-stable parabolic subgroup of $G$ containing $P$ such that $M_{H} \simeq G L_{a} \times S O_{N_{0}}$. Thus $(M \cap P)_{H} \simeq B_{a} \times S O_{N_{0}}$, where $B_{a}$ is a Borel subgroup of $G L_{a}$ such that $B_{H} \cap\left(G L_{a} \times G L_{a}\right)^{\theta} \simeq B_{a}$. Let $\xi=\left(\mathscr{O}_{L}, \mathscr{E}_{1}^{\dagger}\right)$ be a cuspidal pair on $L_{\text {uni }}^{\iota \theta}$, and consider the $L_{H}$-equivariant perverse sheaf $K_{\xi}=\operatorname{IC}\left(\overline{\mathscr{O}}_{L}, \mathscr{E}_{1}^{\dagger}\right)\left[\operatorname{dim} \mathscr{O}_{L}\right]$ on $L^{\iota \theta}$. Since $\operatorname{ind}_{M \cap P}^{M} K_{\xi}$ is isomorphic to $\left(\operatorname{ind}_{B_{a}}^{G L_{a}} K_{0}\right) \otimes K_{\xi}$, where $K_{0}$ is the constant sheaf $\overline{\mathbf{Q}}_{l}$ on $\{1\} \subset T_{a}\left(T_{a}\right.$ is the maximal torus of $\left.B_{a}\right)$, by a well-known result of Borho-MacPherson ([BM]) for $G L_{a}$, $\operatorname{ind}_{M \cap P}^{M} K_{\xi}$ is a semisimple perverse sheaf on $M_{\text {uni }}^{\iota \theta}$, equipped with $S_{a}$-action, and is decomposed as

$$
\operatorname{ind}_{M \cap P}^{M} K_{\xi} \simeq \bigoplus_{\mu \in \mathscr{P}_{a}} \rho_{\mu} \otimes\left(K_{\mu}^{a} \otimes K_{\xi}\right)
$$

where $K_{\mu}^{a}=\operatorname{IC}\left(\overline{\mathscr{O}}_{\mu}^{a}, \overline{\mathbf{Q}}_{l}\right)\left[\operatorname{dim} \mathscr{O}_{\mu}^{a}\right]$ (here $\mathscr{O}_{\mu}^{a}$ is the unipotent class in $G L_{a}$ with Jordan type $\mu$ ). Thus $\operatorname{ind}_{M \cap P}^{M} K_{\xi}$ is a $M_{H}$-equivariant perverse sheaf on $M^{\iota \theta}$, and one can apply the functor $\operatorname{ind}_{Q}^{G}$ on it. By the transitivity of induction (Proposition 10.2), we have

$$
\operatorname{ind}_{P}^{G} K_{\xi} \simeq \bigoplus_{\mu \in \mathscr{P}_{a}} \rho_{\mu} \otimes \operatorname{ind}_{Q}^{G}\left(K_{\mu}^{a} \otimes K_{\xi}\right)
$$

On the other hand, by comparing the construction of $\operatorname{ind}_{P}^{G} K_{\xi}$ with the complex $\pi_{*} K_{\overline{\mathscr{E}}_{1}}$ constructed in 3.7 , we see that $\left.\operatorname{ind}_{P}^{G} K_{\xi} \simeq \pi_{*} K_{\overline{\mathscr{E}}_{1}}[-r]\right|_{X_{\text {uni }}}$. Hence by Theorem 5.2 together with Theorem 9.9, we have

$$
\operatorname{ind}_{P}^{G} K_{\xi} \simeq \bigoplus_{\mu \in \mathscr{P}_{a}} \rho_{\mu} \otimes K_{\Gamma_{\xi}(\mu)}
$$

where $K_{\Gamma_{\xi}(\mu)}=\operatorname{IC}(\overline{\mathscr{O}}, \mathscr{E})[\operatorname{dim} \mathscr{O}]$ if $(\mathscr{O}, \mathscr{E}) \in \mathscr{N}_{G}$ corresponds to $\Gamma_{\xi}(\mu) \in \Psi_{N}$.

$\operatorname{ind}_{Q}^{G}\left(K_{\mu}^{a} \otimes K_{\xi}\right)$ is an $H$-equivariant semisimple complex on $G^{1 \theta}$. Since it is a direct summand of the semisimple perverse sheaf on $G^{\iota \theta}$, it is a semisimple perverse sheaf. By counting the multiplicities $\sum \operatorname{dim} \rho_{\mu}$, we see that $\operatorname{ind}_{Q}^{G}\left(K_{\mu}^{a} \otimes K_{\xi}\right)$ is a simple perverse sheaf on $G_{\text {uni }}^{\iota \theta}$. Hence we have

(10.3.4) $\operatorname{ind}_{Q}^{G}\left(K_{\mu}^{a} \otimes K_{\xi}\right)$ coincides with one of the simple component appearing in the right hand side of (10.3.3).

Remark 10.4 If the isomorphisms in (10.3.2) and (10.3.3) are compatible with $S_{a}$-action, we will immediately get the isomorphism $\operatorname{ind}_{Q}^{G}\left(K_{\mu}^{a} \otimes K_{\xi}\right) \simeq K_{\Gamma_{\xi}(\mu)}$. Note that the diagram in 3.7 with respect to $\pi: \widetilde{X} \rightarrow X$ corresponds to the diagram 
(10.1.1) with respect to $\operatorname{ind}_{P}^{G}$. However, in the case of $\operatorname{ind}_{Q}^{G}$, we cannot construct an analogous diagram of 3.6. So, it is not certain whether $\operatorname{ind}_{Q}^{G}$ commutes with the action of $S_{a}$. Nevertheless we will show in the discussion below that the above isomorphism actually holds.

10.5. Let $\mathscr{O}^{\prime}$ be an $M_{H}$-orbit in $M_{\text {uni }}^{\iota \theta}$. Then there exists a unique $H$-orbit $\mathscr{O}$ in $G_{\text {uni }}^{\iota \theta}$ such that $\mathscr{O} \cap \eta_{Q}^{-1}\left(\mathscr{O}^{\prime}\right)$ is open dense in $\eta_{Q}^{-1}\left(\mathscr{O}^{\prime}\right)$. $\mathscr{O}$ is called the $H$-orbit induced from $\mathscr{O}^{\prime}$, and is denoted by $\mathscr{O}=\operatorname{Ind}_{Q}^{G} \mathscr{O}^{\prime}$. Put

$$
\begin{aligned}
& \widetilde{X}_{\mathscr{O}^{\prime}}=\left\{\left(x, g Q_{H}\right) \in G^{\iota \theta} \times H / Q_{H} \mid g^{-1} x g \in \eta_{Q}^{-1}\left(\overline{\mathscr{O}}^{\prime}\right)\right\} \\
& X_{\mathscr{O}^{\prime}}=\bigcup_{g \in H} g \eta_{Q}^{-1}\left(\overline{\mathscr{O}}^{\prime}\right) g
\end{aligned}
$$

and let $\pi_{\mathscr{O}^{\prime}}: \widetilde{X}_{\mathscr{O}^{\prime}} \rightarrow X_{\mathscr{O}^{\prime}}$ be the first projection. Then $\pi_{\mathscr{O}^{\prime}}$ is proper, surjective. By a similar argument as in the proof of Proposition 8.20, we see that $\mathscr{O}$ is the unique open dense orbit contained in $X_{\mathscr{O}^{\prime}}$. Let $\mathscr{O}_{L}$ be the $L_{H^{-}}$-orbit in $L_{\text {uni }}^{\iota \theta}$ as before, and consider $\mathscr{O}^{\prime}=\mathscr{O}_{\mu}^{a} \times \mathscr{O}_{L}$ for each $\mu \in \mathscr{P}_{a}$. We write $\mathscr{O}^{\prime}=\mathscr{O}_{\mu}^{\prime}$, and express $\pi_{\mathscr{O}^{\prime}}: \tilde{X}_{\mathscr{O}^{\prime}} \rightarrow X_{\mathscr{O}^{\prime}}$ as $\pi_{\mu}: \tilde{X}_{\mu} \rightarrow X_{\mu}$. The following result shows that the induction of $H$-orbits can be realized in the level of perverse sheaves.

Proposition 10.6. Let $\mu \in \mathscr{P}_{a}$. Under the notation above,

(i) $\operatorname{ind}_{Q}^{G}\left(K_{\mu} \otimes K_{\xi}\right) \simeq K_{\Gamma_{\xi}(\mu)}$.

(ii) Let $(\mathscr{O}, \mathscr{E}) \in \mathscr{N}_{G}$ be the pair corresponding to $\Gamma_{\xi}(\mu) \in \Psi_{N}$. Then $\mathscr{O}=$ $\operatorname{Ind}_{Q}^{G}\left(\mathscr{O}_{\mu}^{a} \times \mathscr{O}_{L}\right)$. In particular, the Jordan type of $\mathscr{O}$ is equal to $\nu+2 \mu$.

(iii) $\operatorname{dim} \widetilde{X}_{\mu}=\operatorname{dim} X_{\mu}$.

Proof. First we note the following property, which is obtained from the explicit description of the generalized Springer correspondence in Theorem 9.9 (iii).

(10.6.1) Let $(\mathscr{O}, \mathscr{E}),\left(\mathscr{O}^{\prime}, \mathscr{E}^{\prime}\right) \in \mathscr{N}_{G}$. Assume that $(\mathscr{O}, \mathscr{O})$ corresponds to $\Gamma_{\xi}(\mu)$, and $\left(\mathscr{O}^{\prime}, \mathscr{E}^{\prime}\right)$ corresponds to $\Gamma_{\xi}\left(\mu^{\prime}\right)$. If the Jordan type of $\mathscr{O}$ is the same as that of $\mathscr{O}^{\prime}$, then $\mu=\mu^{\prime}$.

We prove (i) by the backward induction on $n(\mu)$. Assume that the statement holds for $\mu^{\prime}$ such that $n\left(\mu^{\prime}\right)>n(\mu)$. Put $\operatorname{ind}_{Q}^{G}\left(K_{\mu} \otimes K_{\xi}\right)=\operatorname{IC}\left(\mathscr{O}^{\prime}, \mathscr{E}^{\prime}\right)\left[\operatorname{dim} \mathscr{O}^{\prime}\right]$. It follows from the definition of $\operatorname{ind}_{Q}^{G}, \mathscr{O}^{\prime}$ is open dense in $X_{\mu}$. Let $\mathscr{O}_{\mu, \mu, \nu}$ be the unipotent class in $M$ such that its intersection with $M^{\iota \theta}$ coincides with $\mathscr{O}_{\mu}^{\prime}=\mathscr{O}_{\mu}^{a} \times$ $\mathscr{O}_{L}$. Then $\eta_{Q}^{-1}\left(\mathscr{O}_{\mu}^{\prime}\right) \subset \mathscr{O}_{\mu, \mu, \nu} U_{Q}$. It is known from the theory of Hall polynomials (see $[\mathrm{M}]$ ) that the unipotent class in $G L_{N}$ which has open dense intersection with $\mathscr{O}_{\mu, \mu, \nu} U_{Q}$ has Jordan type $2 \mu+\nu$, and all other unipotent classes with non-zero intersection with $\mathscr{O}_{\mu, \mu, \nu} U_{Q}$ have Jordan type $<2 \mu+\nu$. Hence the Jordan type of $\mathscr{O}^{\prime}$ is of the form $2 \mu^{\prime}+\nu \leq 2 \mu+\nu$. By induction hypothesis, for any $\mu^{\prime \prime}$ such that $n\left(\mu^{\prime \prime}\right)>n(\mu)$, the pair $\left(\mathscr{O}_{1}, \mathscr{E}_{1}\right)$ such that $\mathscr{O}_{1}$ has Jordan type $2 \mu^{\prime \prime}+\nu$ is already assigned to $\operatorname{ind}_{Q}^{G}\left(K_{\mu^{\prime \prime}} \otimes K_{\xi}\right)$. Thus $n\left(\mu^{\prime}\right)=n(\mu)$, and so the Jordan type of $\mathscr{O}^{\prime}$ must coincide with $2 \mu+\nu$. Hence $\left(\mathscr{O}^{\prime}, \mathscr{E}^{\prime}\right)$ corresponds to $\Gamma_{\xi}(\mu)$ by $(10.6 .1)$. This proves (i). 
We show (ii) and (iii). It follows from the definition that $\mathscr{O}^{\prime}$ coincides with $\operatorname{Ind}_{Q}^{G} \mathscr{O}_{\mu}^{\prime}$. Thus (ii) follows from (i). We have

$$
\operatorname{dim} X_{\mu}=\operatorname{dim} \mathscr{O}^{\prime}=\operatorname{dim} H-n(\nu+2 \mu)
$$

On the other hand, since $\widetilde{X}_{\mu} \simeq H \times^{Q_{H}} \eta_{Q}^{-1}\left(\overline{\mathscr{O}}_{\mu}^{\prime}\right)$, we have

$$
\operatorname{dim} \tilde{X}_{\mu}=\operatorname{dim} H-\operatorname{dim} Q_{H}+\operatorname{dim} \mathscr{O}_{\mu}^{\prime}+\operatorname{dim} U_{Q}^{\iota \theta}
$$

Since $\operatorname{dim} \mathscr{O}_{\mu}^{\prime}=\left(a^{2}-a-2 n(\mu)\right)+\left(\operatorname{dim} S O_{N_{0}}-n(\nu)\right), \operatorname{dim} U_{Q}^{\iota \theta}=\operatorname{dim} U_{Q}^{\theta}+a$, we conclude that $\operatorname{dim} \widetilde{X}_{\mu}=\operatorname{dim} X_{\mu}$. Thus (iii) holds. The proposition is proved.

Remark 10.7. Proposition 10.6 is a generalization of Proposition 8.20 and of Lemma 9.2. But in contrast to the previous cases, in this discussion, we don't need to show the existence of an element of Jordan type $\nu+2 \mu$ inside of $X_{\mu}$.

\section{APPENDIX}

We give some examples of the generalized Springer correspondence. Here we use a simplified notation to denote the signed partition $(\lambda, \tau)$. If $\lambda=\left(a_{1}^{m_{1}}, a_{2}^{m_{2}}, \ldots\right)$, we only denote the signature \pm 1 corresponding to the block $a_{i}^{m_{i}}$ as \pm . For example, $\left(1^{3} ;+1,+1,+1\right)$ is written as $\left(1^{3} ;+\right)$ and $\left(2^{2} 1^{2} ;-1,-1,+1,+1\right)$ is written as $\left(2^{2} 1^{2} ;-+\right)$. If $c=\left(N_{0}, \nu, \sigma\right) \in \mathscr{C}_{N}$, the set $\Psi_{N}^{(c)}$ is the set of signed partitions corresponding to $\rho_{\mu} \in S_{a}^{\wedge}$ with $a=\left(N-N_{0}\right) / 2$, which we denote by $S_{a} ;(\nu, \sigma)$. Let $\Psi_{N}^{(0)}$ be the set of cuspidal elements in $\Psi_{N}$. In the first column, we only list up the elements which are not contained in $\Psi_{N}^{(0)}$.

- $N=3$.

\begin{tabular}{|c|c|}
\hline$(\lambda, \tau)$ & $S_{1} ;(1 ;+)$ \\
\hline$(3 ;+)$ & $(1)$ \\
\hline
\end{tabular}

$\Psi_{N}^{(0)}=\left\{(21,++),(21 ;-+),\left(1^{3} ;+\right)\right\}$

- $N=5$.

\begin{tabular}{|c|c|ccc|}
\hline$(\lambda, \tau)$ & $S_{2} ;(1 ;+)$ & $S_{1} ;(21 ;++)$ & $S_{1} ;(21 ;-+)$ & $S_{1} ;\left(1^{3} ;+\right)$ \\
\hline$(5 ;+)$ & $(2)$ & & & \\
$(41 ;++)$ & & $(1)$ & & \\
$(41 ;-+)$ & & & & \\
$(32 ;++)$ & $\left(1^{2}\right)$ & & & $(1)$ \\
$\left(31^{2} ;++\right)$ & & & & \\
\hline
\end{tabular}


$\Psi_{N}^{(0)}=\left\{(32 ;+-),\left(31^{2} ;+-\right),\left(2^{2} 1 ;++\right),\left(2^{2} 1 ;-+\right),\left(21^{3} ;++\right),\left(21^{3} ;-+\right),\left(1^{5} ;+\right)\right\}$.

- $N=7$

\begin{tabular}{|c|c|ccc|}
\hline$(\lambda, \tau)$ & $S_{3} ;(1 ;+)$ & $S_{2} ;(21 ;++)$ & $S_{2} ;(21 ;-+)$ & $S_{2} ;\left(1^{3} ;+\right)$ \\
\hline$(7 ;+)$ & $(3)$ & $(2)$ & & \\
$(61 ;++)$ & & & $(2)$ & \\
$(61 ;-+)$ & & & & \\
$(52 ;++)$ & $(21)$ & & & $(2)$ \\
$(52 ;++)$ & & & & \\
$\left(51^{2} ;++\right)$ & & & & \\
$\left(51^{2} ;+-\right)$ & & $\left(1^{2}\right)$ & & \\
$(43 ;++)$ & & & & \\
$(43 ;-+)$ & & & & \\
$(421 ;+++)$ & & & & $\left(1^{2}\right)$ \\
$(421 ;--+)$ & & & & \\
$\left(41^{3} ;++\right)$ & & & & \\
$\left(41^{3} ;-+\right)$ & & & & \\
$\left(3^{2} 1 ;++\right)$ & & & & \\
$\left(32^{2} ;++\right)$ & $\left(1^{3}\right)$ & & & \\
$\left(31^{4} ;++\right)$ & & & & \\
\hline
\end{tabular}

The correspondence in the case of $S_{1}$ is as follows;

$S_{1} ;(32 ;+-) \leftrightarrow(52 ;+-), \quad S_{1} ;\left(31^{2} ;+-\right) \leftrightarrow\left(51^{2} ;+-\right)$,

$S_{1} ;\left(2^{2} 1 ;++\right) \leftrightarrow(421 ;+++), \quad S_{1} ;\left(2^{2} 1 ;-+\right) \leftrightarrow(421 ;--+)$,

$S_{1} ;\left(21^{3}:++\right) \leftrightarrow\left(41^{3} ;++\right), \quad S_{1} ;\left(21^{3} ;-+\right) \leftrightarrow\left(41^{3} ;-+\right)$,

$S_{1} ;\left(1^{5} ;+\right) \leftrightarrow\left(31^{4} ;++\right)$.

Here

$$
\begin{aligned}
\Psi_{N}^{(0)}=\{ & (421 ;-++),(421 ;+-+),\left(3^{2} 1 ;+-\right),\left(32^{2} ;+-\right), \\
& \left(321^{2} ;+++\right),\left(321^{2} ;++-\right),\left(321^{2} ;+-+\right),\left(321^{2} ;+--\right), \\
& \left(31^{4} ;+-\right),\left(2^{3} 1 ;++\right),\left(2^{3} 1 ;-+\right),\left(2^{2} 1^{3} ;++\right),\left(2^{2} 1^{3} ;-+\right), \\
& \left.\left(21^{5} ;++\right),\left(21^{5} ;-+\right),\left(1^{7} ;+\right)\right\} .
\end{aligned}
$$

$\bullet N=2$.

\begin{tabular}{|c|cc|}
\hline$(\lambda, \tau)$ & $S_{1} ;\left(\emptyset^{+} ;+\right)$ & $S_{1} ;\left(\emptyset^{-} ;+\right)$ \\
\hline$\left((2)^{+} ;+\right)$ & $(1)$ & \\
$\left((2)^{-} ;+\right)$ & & $(1)$ \\
\hline
\end{tabular}

$\left.\Psi_{N}^{(0)}=\left\{(2)^{+} ;-\right),\left((2)^{-} ;-\right),\left(1^{2} ;+\right)\right\}$. 
$\bullet N=4$.

\begin{tabular}{|c|cc|ccc|}
\hline$(\lambda, \tau)$ & $S_{2} ;\left(\emptyset^{+} ;+\right)$ & $S_{2} ;\left(\emptyset^{-} ;+\right)$ & $S_{1} ;\left((2)^{+} ;-\right)$ & $S_{1} ;\left((2)^{-} ;-\right)$ & $S_{1} ;\left(1^{2} ;+\right)$ \\
\hline$\left((4)^{+} ;+\right)$ & $(2)$ & & $(1)$ & & \\
$\left((4)^{+} ;-\right)$ & & & & & \\
$\left((4)^{-} ;+\right)$ & & $(2)$ & & & \\
$\left((4)^{-} ;-\right)$ & & & & \\
$(31 ;++)$ & & & & \\
$\left(\left(2^{2}\right)^{+} ;+\right)$ & $\left(1^{2}\right)$ & & & & \\
$\left(\left(2^{2}\right)^{-},+\right)$ & & $\left(1^{2}\right)$ & & & \\
\hline
\end{tabular}

$\Psi_{N}^{(0)}=\left\{(31 ;+-),\left(\left(2^{2}\right)^{+},-\right),\left(\left(2^{2}\right)^{-} ;-\right),\left(21^{2} ;++\right),\left(21^{2} ;-+\right),\left(1^{4} ;+\right)\right\}$.

$\bullet N=6$.

\begin{tabular}{|c|cc|ccc|}
\hline$(\lambda, \tau)$ & $S_{3} ;\left(\emptyset^{+} ;+\right)$ & $S_{3} ;\left(\emptyset^{-} ;+\right)$ & $S_{2} ;\left((2)^{+} ;-\right)$ & $S_{2} ;\left((2)^{-} ;-\right)$ & $S_{2} ;\left(1^{2} ;+\right)$ \\
\hline$\left((6)^{+} ;+\right)$ & $(3)$ & & $(2)$ & & \\
$\left((6)^{+} ;-\right)$ & & & & & \\
$\left((6)^{-} ;+\right)$ & & $(3)$ & & & \\
$\left((6)^{-} ;-\right)$ & & & & \\
$(51 ;++)$ & & & & \\
$(51 ;+-)$ & & & & \\
$\left((42)^{+} ;++\right)$ & $(21)$ & & & \\
$\left((42)^{+} ;-+\right)$ & & & & \\
$\left((42)^{+} ;--\right)$ & & & & \\
$\left((42)^{-} ;++\right)$ & & $(21)$ & & & \\
$\left((42)^{-} ;-+\right)$ & & & & \\
$\left((42)^{-} ;--\right)$ & & & & & \\
$\left(41^{2} ;++\right)$ & & & & & \\
$\left(41^{2} ;-+\right)$ & & & & & \\
$\left(3^{2} ;+\right)$ & & & & & \\
$\left(31^{3} ;++\right)$ & & & & & \\
$\left(\left(2^{3}\right)^{+} ;+\right)$ & $\left(1^{3}\right)$ & & & & \\
$\left(\left(2^{3}\right)^{-} ;+\right)$ & & $\left(1^{3}\right)$ & & & \\
\hline
\end{tabular}

The correspondence in the case of $S_{1}$ is as follows;

$$
\begin{array}{lrl}
(31 ;+-) & \leftrightarrow(51 ;+-), & \left(\left(2^{2}\right)^{+} ;-\right) \leftrightarrow\left((42)^{+} ;--\right), \\
\left(\left(2^{2}\right)^{-} ;-\right) \leftrightarrow\left((42)^{-} ;--\right), & \left(21^{2} ;++\right) \leftrightarrow\left(41^{2} ;++\right), \\
\left(21^{2} ;-+\right) \leftrightarrow\left(41^{2} ;-+\right), & \left.\left(1^{4} ;+\right) \leftrightarrow\left(31^{3}\right) ;++\right) .
\end{array}
$$


Here

$$
\begin{aligned}
\Psi_{N}^{(0)}=\{ & \left((42)^{+} ;+-\right),\left((42)^{-} ;+-\right),(321 ;+++),(321 ;++-), \\
& (321 ;+-+),(321 ;+--),\left(31^{3} ;+-\right),\left(\left(2^{3}\right)^{+} ;-\right),\left(\left(2^{3}\right)^{-} ;-\right), \\
& \left.\left(2^{2} 1^{2} ;++\right),\left(2^{2} 1^{2} ;-+\right),\left(21^{4} ;++\right),\left(21^{4}:-+\right),\left(1^{6} ;+\right)\right\} .
\end{aligned}
$$

\section{REFERENCES}

[BM] W. Borho and R. MacPherson; Représentations des groupes des Weyl et homologie d'intersection pour les variétés nilpotentes, C.R. Acad. Sci. Paris t. 292 (1981), série A, 707-710.

[CVX1] T.H. Chen, K. Vilonen and T. Xue; Springer correspondence for symmetric spaces, preprint, arXiv: $1510.05986 \mathrm{v} 2$.

[CVX2] T.H. Chen, K. Vilonen and T. Xue; Springer correspondence for the split symmetric pair in type $A$, preprint, arXiv:1608.06034v1.

[H] A. Henderson; Fourier transform, parabolic induction, and nilpotent orbits, Transformation Groups 6 (2001), 353 - 370.

[K] S. Kato; An exotic Deligne-Langlands correspondence for symplectic groups, Duke Math. J. 148 (2009), 306 - 371.

[KR] B. Kostant and S. Rallis; Orbits and representations associated with symmetric spaces, Amer. J. Math. 93 (1971), 753 - 809.

[L1] G. Lusztig; Intersection cohomology complexes on a reductive group, Invent. Math.75 (1984), 205-272.

[L2] G. Lusztig; Character sheaves, I, Adv. in Math. 56 (1985), 193 - 237.

[LS] G. Lusztig and N. Spaltenstein; On the generalized Springer correspondence for classical groups, in Algebraic Groups and Related Topics, Adv. Studies in Pure Math. 6, (1985), pp.289 - 315 .

[LY] G. Lusztig and Z. Yun; Z/ $m$-graded Lie algebras and perverse sheaves, I, Representation Theory 21 (2017), 277-321, II, ibid. 21 (2017), 322-353, III, Graded double affine Hecke algebra, ibid. 22 (2018), 87-118, IV, preprint, arXiv: 1805.10550.

[M] I.G. Macdonald; "Symmetric functions and Hall polynomials", Clarendon Press, Oxford, 1995.

[R] R.W. Richardson; Orbits, invariants, and representations associated to involutions of reductive groups, Inv. Math. 66 (1982), 287-312.

[St] R. Steinberg; Endomorphisms of linear algebraic groups, Mem. Amer. Math. Soc. 80, (1968).

[SS] T. Shoji and K. Sorlin; Exotic symmetric space over a finite field, I, Transformation Groups, 18 (2013), 877 - 929.

[V] T. Vust; Opération de groupes rèductifs dans un type de cônes presque homogenes, Bull. Soc. Mat. France 102 (1974), 317-334.

\section{T. Shoji}

School of Mathematical Sciences, Tongji University

1239 Siping Road, Shanghai 200092, P. R. China

E-mail: shoji@tongji.edu.cn 
G. Yang

Department of Mathematics, Harbin Engineering University

145 Nantong Street, Harbin 150001, P.R. China

E-mail: yanggao_670206@163.com 